\title{
The Hintons' legacy to the KNOWLedge of the floRA of MeXico
}

\section{El LEGAdo de los Hinton al CONOCIMIENTO dE LA FLORA DE MÉXICO}

\author{
GeORge S. Hinton ${ }^{1}$, José LUIS VILLASEÑoR ${ }^{2 *}$, AND ENRIQUe ORTIZ²
}

\author{
${ }^{1}$ Rancho Aguililla, Galeana, Nuevo León, México. \\ 2 Instituto de Biología, Universidad Nacional Autónoma de México, Departamento de Botánica, Ciudad de México, México. \\ *Correspondig author: vrios@ib.unam.mx
}

\begin{abstract}
Background: The Hinton family comprises three generations of plant collectors that have made an important contribution to the knowledge of the flora of Mexico. They have collected in 13 Mexican states and have provided material for the description of 6 genera, 621 species and 51 infraspecific taxa new to science.

Question: What is the contribution of the collections of the Hintons to our knowledge of the flora of Mexico? Where was their main collecting effort and how many species have been recorded?

Species study: Vascular plants.

Study site: Mexico.

Method: Different national and foreign databases were consulted and a list of species collected by the Hintons was obtained. The records were reviewed to eliminate synonymy and a complete list of species and their distribution by states was compiled.

Results: A total of 28,947 records were obtained and 25,717 of them were identified to species level. In addition to the family's collection, the herbaria with the largest number of records are LL-TEX, K, IEB, and MEXU. The records come from about 1,654 different collecting localities throughout Mexico and account for 5,730 species, 1,573 genera, and 233 families.

Conclusions: Many of the specimens collected by the Hintons come from areas that are still underexplored today. The contribution of the Hinton family to the knowledge of the flora of Mexico represents an important legacy and their collections constitute an enduring source of information for people interested in the flora of Mexico.
\end{abstract}

Key words: Botanical collections, botanical exploration, databases, floristics, Mexican collectors.

\section{Resumen}

Antecedentes: La familia Hinton comprende tres generaciones de botánicos colectores que han hecho una contribución substancial al conocimiento de la flora de México. Sus recolectas provienen de 13 estados mexicanos y han servido para la descripción de 6 géneros, 621 especies y 51 taxa infraespecíficos nuevos para la ciencia.

Pregunta: ¿Cuál es la contribución de las recolectas de los Hinton a la flora de México? ¿Dónde ha estado su principal esfuerzo de recolección y cuántas especies han registrado?

Especies de estudio: Plantas vasculares.

Sitio de estudio: México.

Métodos: Se consultaron diferentes bases de datos nacionales y extranjeras y se obtuvo una lista de especies recolectadas por los Hinton. Los registros fueron revisados para eliminar la sinonimia y se compiló una lista de las especies y su distribución por estados.

Resultados: Se obtuvieron un total de 28,947 registros y 25,717 de ellos se identificaron completamente a nivel de especie. Además de la colección de la familia, los herbarios con el mayor número de registros son LL-TEX, K, IEB y MEXU. Los registros provienen de aproximadamente 1,654 localidades diferentes a lo largo de México y cuentan con 5,730 especies, 1,573 géneros y 233 familias.

Conclusiones: Muchos de los especímenes recolectados por los Hinton provienen de áreas todavía poco exploradas actualmente. La contribución de la familia Hinton al conocimiento de la flora de México constituye un importante legado y sus colecciones constituirán durante muchos años un conjunto de información muy importante para las personas interesadas en la flora de México.

Palabras clave: Bases de datos, colecciones biológicas, colectores mexicanos, exploración botánica, florística. 
In 1972, Jaime Hinton and Jerzy Rzedowski published the biography of George Boole Hinton (1882-1943, Figure 1), a botanical collector born in London, England (a spanish version of this contribution was published in 1975). These authors point out that in 1911 he arrived in Mexico and after working for many years as a metallurgist, at the age of 54 he retired from mining to devote himself to collecting botanical specimens. During ten years of intense activity of fieldwork, Hinton managed to make a collection of just over 16,000 numbers from difficult and unexplored localities in the states of Guerrero, México, and Michoacán. In his biography, Hinton and Rzedowski relate the difficulties encountered and the effort required to amass one of the best collections of regions of Mexico that to this day persist as scientifically underexplored due to the ruggedness of the topography and to social and political unrest.

Fondness for collecting botanical specimens was also acquired by George's son James (1915-2006, Figure 1), who in July 1937 joined his father for fieldwork in the state of Guerrero. He soon became one of the main collectors of Mexican plants; after his father's death he continued collecting specimens in other parts of the country, especially Coahuila, Nuevo León, Oaxaca, and Tamaulipas. Table 1 indicates the number of collections carried out in these states based on the collections of George and James Hinton. Later, when James was living in the state of Nuevo León, his son George Sebastian (Figure 1) began accompanying James on his botanical explorations. Since then, George S. has continued this tradition, building up one of the largest personal scientific collection documenting the flora of northeastern Mexico, with the discovery of many new taxa. Based on all these collections, 6 genera and 621 new species were discovered.
This paper aims to document and analyze the entire Hinton collection in order to evaluate its contribution to our knowledge of the flora of Mexico. As can be seen in Table 1 , there are three main periods when the Hinton family (the grandfather, the son, and the grandson) carried out fieldwork, mostly restricted to specific regions in the country. The role of each set of specimens is evaluated and their contribution to the knowledge of the regional and national flora is discussed. Finally, a list of the accepted species and the collection numbers documenting their occurrence is provided. The material that forms the basis of the descriptions of the new taxa is highlighted.

A short story of the collecting effort of the Hinton family. George B. Hinton started fieldwork on October $11^{\text {th }}$, 1931. His first plant collected was Elaphoglossum erinaceum (Fée) T. Moore in the La Labor, District (now Municipality) of Temascaltepec, state of México. His last collection was number 16,314 on January 31, 1943 from the Los Hornos, District of Temascaltepec. In that period he was assisted by Luis Martínez and Gerónimo García, natives of the region. On July 23, 1937, his son James left his studies in Canada to help his father full time in his collecting enterprise. James started his collecting in the state of Guerrero. This first period accounts for about 16,000 collecting numbers and resulted in the description of 471 new taxa.

After his father's death in 1944 James collected intermittently in Coahuila, Nuevo León, and Morelos. In 1964 he started collecting again in the alpine areas of southeastern Nuevo León and adjacent Coahuila. He also collected in alpine regions of Tamaulipas and later in Oaxaca. James continued the numbering of his father and his last collecting

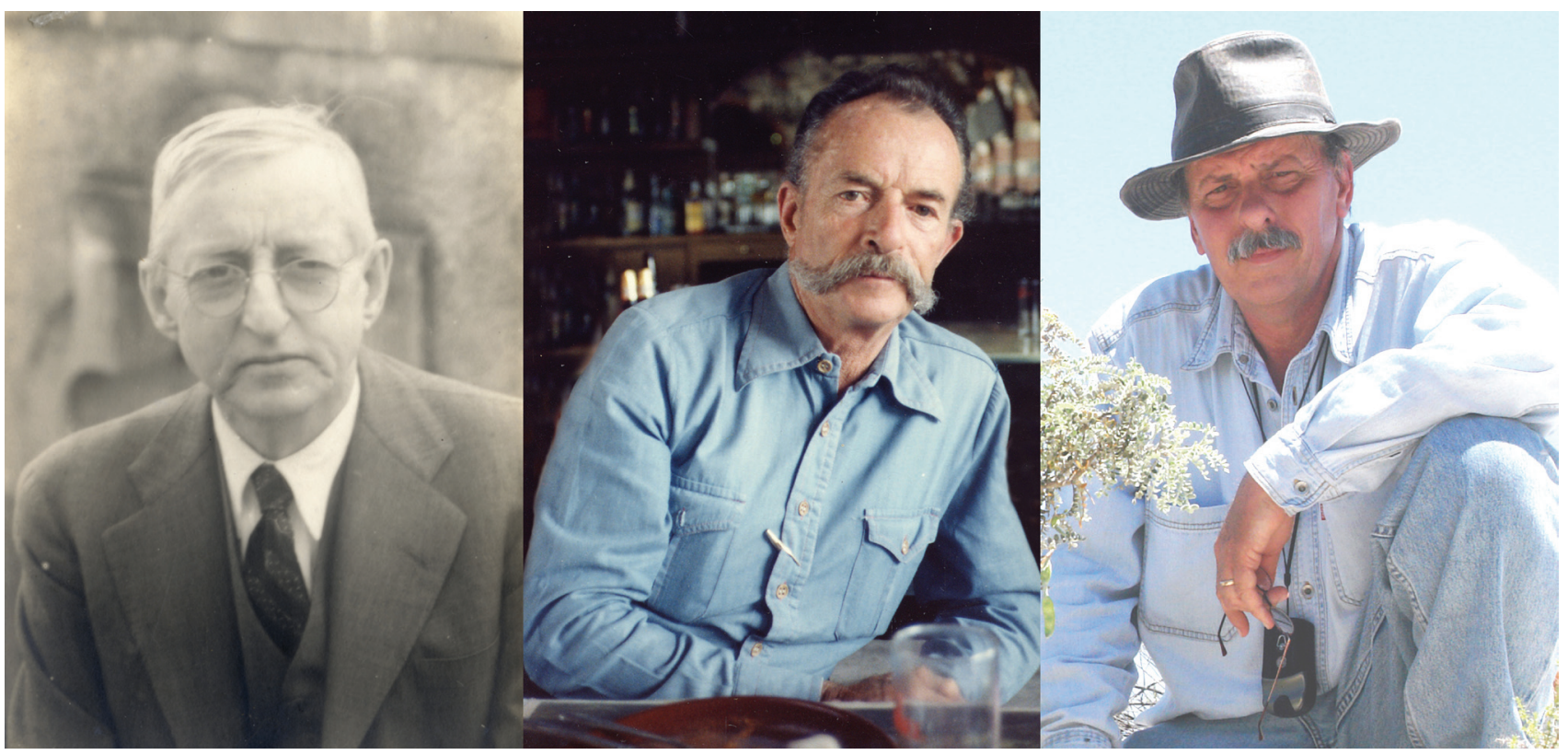

Figure 1. From left to right: George Boole Hinton, James Hinton, and George Sebastian Hinton. 
Table 1. Principal states where the Hinton family carried out fieldwork for collecting botanical specimens in Mexico.

\begin{tabular}{lcc}
\hline Collector & State & $\begin{array}{c}\text { Number of } \\
\text { collections }\end{array}$ \\
\hline George B. Hinton and James Hinton & Guerrero & 4,617 \\
George B. Hinton and James Hinton & México & 7,681 \\
George B. Hinton and James Hinton & Michoacán & 3,585 \\
James Hinton and George S. Hinton & Coahuila & 2,155 \\
James Hinton & Morelos & 178 \\
James Hinton and George S. Hinton & Nuevo León & 8,780 \\
James Hinton & Oaxaca & 855 \\
James Hinton & Tamaulipas & 605 \\
George S. Hinton & San Luis Potosí & 94 \\
\hline
\end{tabular}

was recorded on October 14, 1995 (GBH 26,855). During this time, he collected 7,227 numbers, which included 116 new taxa.

James' son George Sebastian started collecting plants in 1989; with his father he made 1,183 collections and 6,712 on his own. After his father's death, he has continued to conduct fieldwork, mostly in the states of Coahuila and Nuevo León, and has also continued the numbering started by his grandfather. One genus and 34 new taxa have been described from these collections. To date, the Hintons' collection catalogues almost 29,000 collection numbers.

\section{Materials and methods}

The list of species collected by the Hinton family was obtained mainly from the database created and administered by George S. Hinton for their own collections. The records contained in the database were complemented and compared with two other important databases, the National Biodiversity Information System (SNIB-REMIB) maintained by the Comisión Nacional para el Conocimiento y Uso de la Biodiversidad (CONABIO), and the digital repository of the National Herbarium of Mexico (MEXU-UNIBIO), maintained by the Instituto de Biología, Universidad Nacional Autónoma de México (UNAM).

Once the three databases were fused, a list of species and the collection numbers supporting each scientific name was obtained. The list of names was compared with the national checklist published by Villaseñor (2016) and with the catalogs of Tropicos (http://www.tropicos.org) and The International Plant Names Index (http://www.ipni.org/ now http://www.plantsoftheworldonline.org/) in order to cite the most currently accepted name and to eliminate the names considered synonyms. The entire list of species is provided in the Appendix 1. The Appendix 2 (see Supplementary Material) includes the same list but ordered by collecting numbers. When a collection number represents the voucher from which a new taxon was described, it is indicated with the word TYPUS.

\section{Results}

The database built by George S. Hinton summarizes all the collection effort carried out during three generations of his family (Table 1). It accounts for 28,947 unique records collected from 1931 to 2018 (Appendix 1). This figure includes 25,717 records fully identified (to species level) and 3,229 unidentified. This latter figure includes 213 records with uncertain, unresolved names. Table 2 summarizes the current status of the records organized by main botanical groupings. The angiosperms constitute the best represented group, particularly the dicots (Magnoliopsida) with the largest number of records, followed by the monocots and the ferns. The database includes 26 additional records of non-vascular plants (Cryptogams).

The revision of the different databases (GBH, Hinton's personal collection), SNIB, and UNIBIO) resulted in the combination of a total of 71,136 records that document the Hinton collections stored in more than 40 herbaria, either national or foreign. Table 3 indicates the number of specimens stored in the 10 herbaria with the largest number of records. Without considering the private collection (GBH),

Table 2. Status of the records in the Hinton collections database arranged by main taxonomic groups.

\begin{tabular}{lcc}
\hline Taxonomic group & $\begin{array}{c}\text { Number of } \\
\text { records }\end{array}$ & $\begin{array}{c}\text { Fully } \\
\text { identified }\end{array}$ \\
\hline Unidentified & 3,229 & \\
Cryptogams & 26 & 19 \\
Ferns and allies & 1,064 & 1,055 \\
Gymnosperms & 192 & 175 \\
Liliopsida (Monocots) & 2,884 & 2,718 \\
Magnoliopsida (Dicots) & 22,862 & 21,762 \\
\hline
\end{tabular}

Table 3. The 10 most important herbaria with fully identified and digitized specimens collected by the Hinton family. Source of data: George S. Hinton's private database; SNIB of CONABIO, and UNIBIO of the Instituto de Biología, UNAM.

\begin{tabular}{lcc}
\hline Herbarium & Records & Species \\
\hline GBH & 25,717 & 5,729 \\
LL-TEX & 10,704 & 3,226 \\
K & 8,409 & 1,372 \\
IEB & 4,797 & 1,826 \\
MEXU & 4,023 & 1,655 \\
NY & 2,632 & 880 \\
ARIZ & 1,760 & 885 \\
US & 1,369 & 533 \\
ENCB & 1,203 & 691 \\
MO & 661 & 364 \\
Other herbaria & 5,432 & \\
\hline
\end{tabular}


the herbaria with the most records (LL-TEX and K) have been involved in an important way in the identification of the material collected. An important account of the Fabaceae (Leguminosae) collected by G. B. Hinton and housed at K was published by Rico-Arce et al. (2008), who report 1,537 specimens including 85 genera and 415 species.
Figure 2 shows the localities where the plant specimens were collected. The western parts of the states of Guerrero, México, and Michoacán concentrate most of the collecting effort. Another important collecting site is located in southern Oaxaca, especially in the District of Miahuatlán where at least 15 new taxa were discovered (Table 1). The last

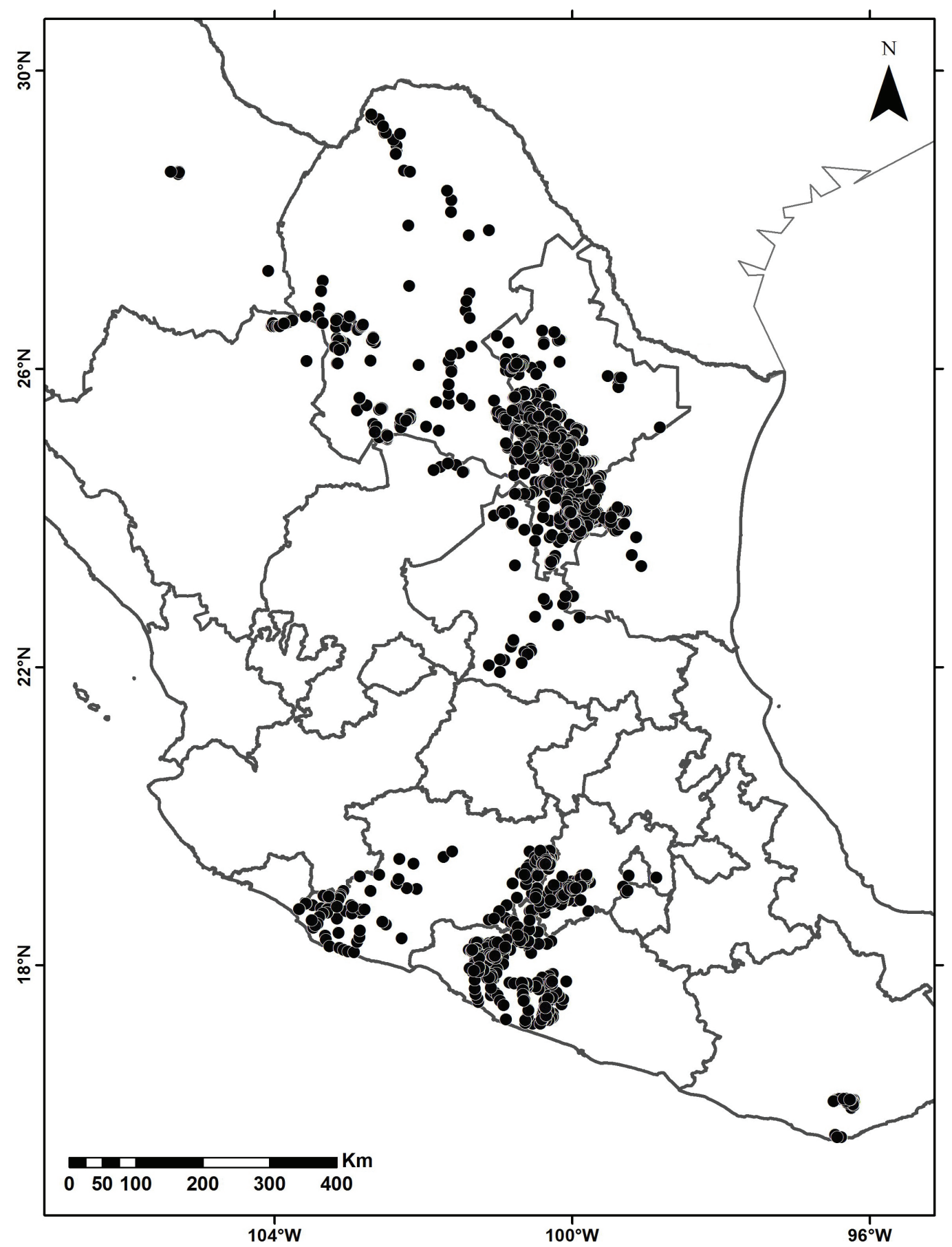

Figure 2. Distribution in Mexico of the collecting points by the Hinton family. 
Table 4. Number of fully identified records, species, and new taxa (between parenthesis those actually accepted) described by state collected in the three main collecting periods by the Hinton family in Mexico. I = 1931-1943; II = 1944-1995; III = 1996 to present. Figures correspond to the records included in the Hintons' private database $(\mathrm{N}=25,717)$. The main Mexican biomes and their percentages of records collected are indicated: HMF $=$ Humid mountain forest; HTF = Humid tropical forest; SDTF $=$ Seasonally dry tropical forest; TEMF $=$ Temperate forest; XERS $=$ Xerophytic scrub.

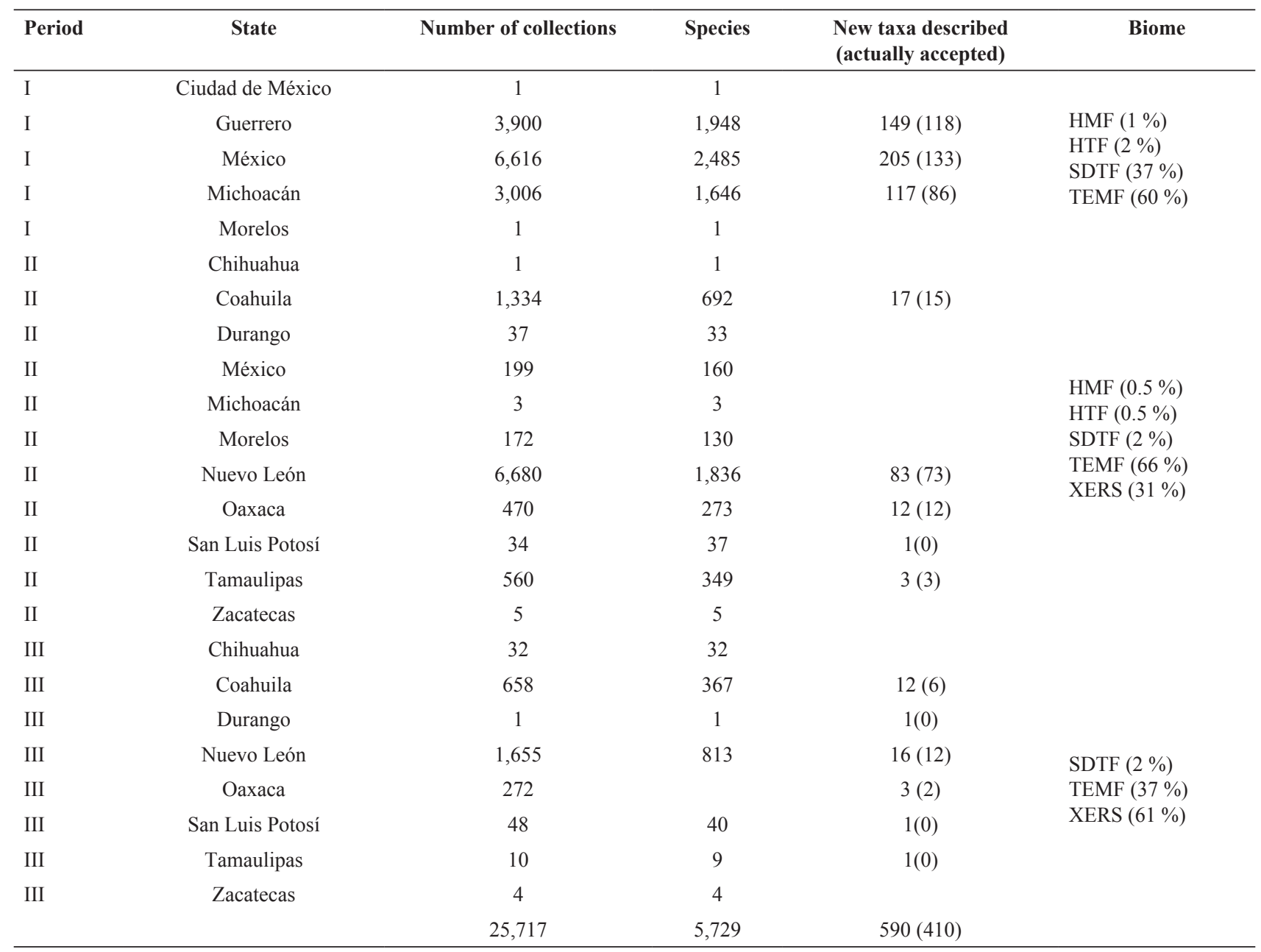

important region where an intense collecting effort has been carried out is the southern half of the states of Coahuila and Nuevo León in northeastern Mexico. Tables 1 and 4 summarize the information about the collecting effort by each member of the Hinton family and the states where fieldwork has been carried out.

Table 4 shows the distribution of the records fully identified both by state and by number of collections $(\mathrm{N}=25,717)$. The collecting effort was divided according to the three main periods of activities and people involved (I: George B. Hinton, George B. and James; II: James; III: James and George S., George S.). In each period the states collected are indicated as well as the number of records and species. It is likewise indicated the number of collections that constitute type material and represent new taxa to science.

The specimens collected come from all the five main biomes in Mexico (Villaseñor \& Ortiz 2014). Table 4 indicates the percentages of specimens collected in each biome by period. The main biomes where most collections come from are temperate forests, seasonally dry tropical forests, and xerophytic scrubs. The first collecting period was concentrated in the temperate and dry forests from western Mexico (Guerrero, México, and Michoacán); during the second the effort continued in the environments of the first but also concentrated in the xerophytic scrub of northeastern Mexico. The third has put much effort in collecting the dry environments of northeastern Mexico.

The database indicates that the specimens come from about 1,654 different collecting points, distributed in 16 out of 32 Mexican states (Figure 2, Table 5). The largest number of records come from the state of Nuevo León, accounting for 827 different collecting stations. The next largest number was made in the state of México, where many records were obtained although from a lesser number of stations. Table 
Table 5. Number of records and unique localities where the Hintons fully identified specimens were collected, distributed among the Mexican states. Figures correspond to the records included in the Hinton's private database $(\mathrm{N}=25,717)$.

\begin{tabular}{lcc}
\hline State & Records & Localities \\
\hline Nuevo León & 8,337 & 827 \\
México & 6,815 & 102 \\
Guerrero & 3,900 & 212 \\
Michoacán & 3,009 & 137 \\
Coahuila & 2,001 & 235 \\
Oaxaca & 742 & 39 \\
Tamaulipas & 572 & 45 \\
Morelos & 174 & 3 \\
San Luis Potosí & 84 & 28 \\
Durango & 38 & 13 \\
Chihuahua & 33 & 4 \\
Zacatecas & 9 & 8 \\
Ciudad de México & 1 & 1 \\
\hline
\end{tabular}

5 shows the states where the specimens were obtained, the total number of records, and the number of different localities visited.

In total, the Hinton database records 5,730 species of vascular plants, distributed in 1,573 genera and 223 families. Table 5 indicates the best represented families and genera recorded in the database. It includes 6 genera and 621 species new to science described based on their collections; this figure does not include 65 additional taxa described with their collections at infrapecific level (subspecies or variet- ies). The six new genera are Cremastopus Paul G. Wilson (Cucurbitaceae, now a synonym of Cyclanthera), Geohintonia Glass \& W.A. Fitz Maur. (Cactaceae), Hintonella Ames (Orchidaceae), Hintonia Bullock (Rubiaceae), Onocleopsis Ballard (Aspidiacae), and Symplococarpon Airy-Shaw (Theaceae).

\section{Discussion}

Among the most important botanical collectors of the 20th century, the Hinton family stands out for their great number of collections. Together they are among the 10 most prolific collectors in Mexico (Villaseñor, unpublished data) and undoubtedly rank first or second by their number of discoveries new to science. But more importantly, the regions where they collected remain inadequately explored, so that their collections represent for many taxa the only references that document their occurrence.

An important number of records remain in need of identification. A figure of 1,898 records lack placement among higher taxonomic groups (Table 2); in addition, 255 records are only identified to family level, and 863 records are identified to genus, still waiting their identification to species level. In addition, 213 records were left out because their determination was considered dubious, in need of corroboration.

Until now a figure of 6 genera and 621 new species have been described based on the Hintons' collections (Table 4, Appendix 1). The current accepted taxonomy does recognize 460 of them as accepted species and 161 as synonyms. In addition, 65 new taxa at infraspecific level also have been described form their plant material (not included in this analysis). In particular, for the family Asteraceae, from a total of 3,825 specimens reviewed that constitute type material (Villaseñor, unpublished data), 188 new taxa have been described

Table 6. The 15 families and genera (and their number of species) best represented in the Hinton's collection.

\begin{tabular}{|c|c|c|c|c|c|}
\hline Family & Records & Species & Genus & Records & Species \\
\hline Asteraceae & 4,462 & 848 & Quercus & 619 & 51 \\
\hline Fabaceae & 2,418 & 570 & Salvia & 609 & 115 \\
\hline Lamiaceae & 1,092 & 210 & Bursera & 425 & 41 \\
\hline Poaceae & 969 & 309 & Euphorbia & 326 & 73 \\
\hline Malvaceae & 686 & 150 & Ipomoea & 324 & 62 \\
\hline Fagaceae & 619 & 51 & Ageratina & 283 & 46 \\
\hline Orchidaceae & 591 & 200 & Stevia & 216 & 29 \\
\hline Euphorbiaceae & 590 & 157 & Cuphea & 211 & 31 \\
\hline Solanaceae & 524 & 127 & Dalea & 200 & 41 \\
\hline Convolvulaceae & 493 & 103 & Solanum & 189 & 50 \\
\hline Rubiaceae & 467 & 92 & Castilleja & 184 & 16 \\
\hline Apocynaceae & 447 & 108 & Verbesina & 184 & 38 \\
\hline Pteridaceae & 440 & 97 & Lupinus & 168 & 29 \\
\hline Burseraceae & 425 & 41 & Muhlenbergia & 162 & 43 \\
\hline Verbenaceae & 398 & 61 & Brickellia & 154 & 26 \\
\hline
\end{tabular}


with specimens collected by them. This number places the Hintons in third place among those who have provided the most material for the description of new taxa, only after $\mathrm{C}$. G. Pringle (397) and E. Palmer (272).

The collecting effort carried out by the Hintons represent an important contribution to state floras and the biomes included (Table 4). Compared with the figures provided by Villaseñor (2016), the number of species recorded in the database for Guerrero includes $29.7 \%$ of the state's flora. Likewise, it includes $42.3 \%$ of the flora of the state of México, $28 \%$ of the flora of Michoacán, $23.5 \%$ of the flora of Coahuila, and $53.6 \%$ of the flora of Nuevo León. As mentioned before, many of the specimens collected by the Hintons in these states represent the only information available about the biology of the species recorded.

Several areas where the Hintons made efforts in collecting plants remain unexplored or floristically poorly known. For example, the Municipality of Temascaltepec in the state of Mexico was the area where George B. Hinton started his collecting effort; 2,061 species have been recorded there (Villaseñor, unpublished data) and the Hintons' database records $1,039(50.4 \%)$ of these species. In contrast, 804 species are recorded for the Municipality of Luvianos, also in the state of Mexico, (Villaseñor, unpublished data), 618 (76.9\%) of which were collected by GB Hinton. Another region that stands out is southern Nuevo León, where most of the flora known there comes from the Hintons' effort. For the entire state, 3,740 species have been reported (Villarreal-Quintanilla \& Estrada-Castillón 2008, Villaseñor 2016); the Hintons' effort has recorded 2,003 of these species, representing $53.6 \%$ of the state's flora. The Hintons are presently the most important collectors for the southern part of the state, and a first account of the state's floristic diversity has been published (Hinton \& Hinton 1995).

The paper by Rico-Arce et al. (2008) provides the opportunity to make a comparison between the information available at the Kew herbarium $(\mathrm{K})$ and the records reported here. At $\mathrm{K}$ the authors indicate there are 1,537 records of Fabaceae (Leguminosae); this figure represents $18.3 \%$ of the records for the Hintons housed at K (Table 3). Likewise, they represent about $63.6 \%$ of the total records for the Fabaceae in the database described here. Finally, the 415 species of Fabaceae identified by the authors account for 72.8 $\%$ of the total species collected by the Hintons. Rico-Arce et al. (2008) point out the important role that $\mathrm{K}$ had in the identification and housing of the collection generated by the Hinton family.

There is no doubt that the contribution of the Hinton family to the knowledge of the flora of Mexico constitutes

\section{Associated Editor: Salvador Arias}

Authors contribution: JLV and GSH conceived the idea of the paper, performed the analysis and wrote the manuscript. EO systematized the databases, managed the information, made the tables and figure and revised the manuscript.

Sunnlementary material: Appendix 2 an important legacy. Of individuals collectors that likewise have made important contributions to this effort, such as E. Palmer, C. G. Pringle, R. McVaugh, or J. Rzedowski, no other example like that of the Hintons exists in Mexico (and perhaps in other parts of the world) where at least three generations have been involved in continuing the important role of collecting botanical specimens. Their collections will for many years constitute an important source of information for all interested in the flora of Mexico.

An important number of specimens $(11,804)$ from the Hintons' collection can be reviewed at the Irekani webpage of the Instituto de Biología, Universidad Nacional Autónoma de México (http://unibio.unam.mx/irekani/ ?locale $=$ es\&proyecto =Irekani\&column=2). The same set of pictures can also be observed at the search-by-species web-page of Tropicos maintained by the Missouri Botanical Garden.

\section{Acknowledgments}

We thank the SNIB (Sistema Nacional de Información sobre Biodiversidad) of the Comisión Nacional para el Conocimiento y Uso de la Biodiversidad and the Instituto de Biología, through its UNIBIO (Unidad de Informática para la Biodiversidad) for allowing the use of the information stored in their databases. David S. Gernandt and Patricia Hinton Davison read the manuscript and made important suggestions to improve its content.

\section{Literature cited}

Hinton J, Hinton GS. 1995. Checklist of Hinton' collections of the flora of south-central Nuevo León and adjacent Coahuila. Acta Botanica Mexicana 30: 41-112.

DOI: https://doi.org/10.21829/abm30.1995.732

Hinton J, Rzedowski J. 1972. George B. Hinton, collector of plants in southwestern Mexico. Journal of the Arnold Arboretum 53: 141-181.

Hinton J, Rzedowski J. 1975. George B. Hinton, explorador botánico en el sudoeste de México. Anales de la Escuela Nacional de Ciencias Biológicas 21: 1-114.

Rico-Arce ML, Rodríguez A, Moreno-Gutiérrez E. 2008. Las colecciones de Leguminosas efectuadas por George B. Hinton, depositadas en el herbario de Royal Botanic Gardens, Kew. Acta Botanica Mexicana 84: 73-92. DOI: https://doi.org/10.21829/abm84.2008.1067

Villarreal-Quintanilla JA, Estrada-Castillón E. 2008. Flora de Nuevo León. Listados Florísticos de México XXIV. México City: Instituto de Biología, Universidad Nacional Autónoma de México.

Villaseñor JL. 2016. Checklist of the native vascular plants of Mexico. Revista Mexicana de Biodiversidad 87: 559-902. DOI: https://doi.org/10.1016/j.rmb.2016.06.017

Villaseñor JL, Ortiz E. 2014. Biodiversidad de las plantas con flores (División Magnoliophyta) en México. Revista Mexicana de Biodiversidad Suppl. 85: S134-S142. DOI: https://doi.org/10.7550/rmb.31987 
Appendix 1. List of species collected by the Hinton family including first the plant group (ferns and lycophytes, gymnosperms and angiosperms), the family and the collecting number. When the collection number represents the voucher for the description of a new taxon, the word TYPUS is added. Some additional vouchers were used to describe new taxa, but now these names are considered as synonyms; they are indicated in the catalog as "Type of ." Likewise, when a voucher was used to describe an infraspecific taxon, it is also indicated as "Type of.".

\section{Ferns and Lycophytes}

\section{Family Anemiaceae}

Anemia adiantifolia (L.) Sw. 6299, 7258, 25321.

Anemia hirsuta (L.) Sw. 1640, 1790, 1999, 5838, 14490, 15214.

Anemia jaliscana Maxon. 1935 (Type of Anemia jaliscana var. integrifolia Mickel), 12212.

Anemia mexicana Klotzsch. 17709, 20058.

Anemia oblongifolia (Cav.) Sw. 2166, 8221, 9472.

Anemia pastinacaria Moritz ex Prantl. 11336, 14650.

Anemia phyllitidis (L.) Sw. 2185, 2489, 5117, 9875, 10704 , 12316, 14636, 14722.

Anemia tomentosa (Savigny) Sw. 12, 1653, 2077, 2141, 2683, 7347, 11338 .

\section{Family Aspleniaceae}

Asplenium blepharodes D.C. Eaton. 2746, 6847, 26275.

Asplenium castaneum Schltdl. \& Cham. 17189, 26142.

Asplenium cristatum Lam. 2389, 5596, 7472, 8941.

Asplenium formosum Willd. 9561.

Asplenium fragrans Sw. 2390, 7803, 10732, 12342, 14230, 15611, 17062, 26371.

Asplenium monanthes L. 7, 34, 1525, 6835, 6838, 7345, $7377,11111,11112,11117,11118,11119,11131,15373$, 15671, 17374, 19454, 21293, 22391, 22541, 25113, 26111, 26162, 26511.

Asplenium myriophyllum (Sw.) C. Presl. 6849, 14302.

Asplenium potosinum Hieron. 14705.

Asplenium praemorsum Sw. 4, 1251, 4158, 14313, 17061.

Asplenium pumilum Sw. 1835.

Asplenium resiliens Kunze. 16921, 17072, 17075, 19060, 19367, 19403, 19709, 20077, 20865, 20974, 21030, 21093, 21292, 22685, 25080, 27791.

Asplenium serra Langsd. \& Fisch. 14279.

Asplenium sessilifolium Desv. 3546, 7360.

Asplenium ultimum A.R. Sm. 20142 (TYPUS).

\section{Family Athyriaceae}

Athyrium skinneri (Baker) Diels. 2649, 4841bis, 4941, 9626, 10631, 10930, 16171.

Diplazium cristatum (Desr.) Alston. 4225, 7543bis.

Diplazium franconis Liebm. 2768.

Diplazium lonchophyllum Kunze. 14048, 14210, 16258.

Family Blechnaceae

Blechnum ensiforme (Liebm.) C. Chr. 14512.
Blechnum falciforme (Liebm.) C. Chr. 14207, 14304, 26040.

Blechnum gracile Kaulf. 11039.

Blechnum occidentale L. 2, 35, 2572, 3461, 7325, 10100, 11309, 11660, 13443, 14049, 15672, 16264, 17059.

Blechnum polypodioides Raddi. 3265.

Blechnum stoloniferum (Mett. ex E. Fourn.) C. Chr. 7222.

Woodwardia radicans (L.) Sm. 40, 1324.

Woodwardia spinulosa M. Martens \& Galeotti. 4103, 4104, 4130, 10162, 15610, 17071, 17090, 25279.

\section{Family Cyatheaceae}

Alsophila firma (Baker) D.S. Conant. 3629.

Cyathea bicrenata Liebm. 14185.

Cyathea costaricensis (Mett. ex Kuhn) Domin. 10793, 16262.

\section{Family Cystopteridaceae}

Cystopteris fragilis (L.) Bernh. 28, 11121, 13499, 15711, 17047, 17304, 17392, 17521bis, 17971, 21290, 21299, 24024.

\section{Family Dennstaedtiaceae}

Dennstaedtia cicutaria (Sw.) T. Moore. 16254, 16267.

Dennstaedtia distenta (Kunze) T. Moore. 3637, 3748, 3776, 7372, 14046, 14187.

Pteridium aquilinum (L.) Kuhn. 21269, 22135.

Pteridium arachnoideum (Kaulf.) Maxon. 14069, 14083.

Pteridium caudatum (L.) Maxon. 6155, 7371.

\section{Family Dryopteridaceae}

Bolbitis portoricensis (Spreng.) Hennipman. 11050, 14679, 16305.

Ctenitis equestris (Kunze) Ching. 7361.

Dryopteris wallichiana (Spreng.) Hyl. 26746.

Dryopteris cinnamomea (Cav.) C. Chr. 3447, 3469, 7316, 15639, 16040, 16155.

Dryopteris karwinskyana (Mett.) Kuntze. 1501, 1818, 11467.

Dryopteris maxonii Underw. \& C. Chr. 9445, 12211.

Dryopteris patula (Sw.) Underw. 41, 1252, 4545, 9344.

Dryopteris pseudofilix-mas (Fée) Rothm. 22097.

Dryopteris wallichiana (Spreng.) Hyl. 17, 2749, 3555, 6846, 14781, 15358, 15709, 17067, 17088, 26077, 26103.

Elaphoglossum affine (M. Martens \& Galeotti) T. Moore. 3275, 13459.

Elaphoglossum erinaceum (Fée) T. Moore. 1, 1867, 15609. Elaphoglossum glaucum T. Moore. 15372.

Elaphoglossum laxipes Mickel. 2750 (TYPUS).

Elaphoglossum lindenii (Bory ex Fée) T. Moore. 7221, 8934, 14295.

Elaphoglossum peltatum (Sw.) Urb. 10756, 11072, 14273.

Elaphoglossum petiolatum (Sw.) Urb. 1906, 13445, 15613, 22679.

Elaphoglossum piloselloides (C. Presl) T. Moore. 8205.

Elaphoglossum potosianum C. Chr. 22119.

Elaphoglossum sartorii (Liebm.) Mickel. 14303.

Elaphoglossum tenuiculum (Fée) T. Moore ex C. Chr. 8241, 
14882, 15368, 24582.

Megalastrum subincisum (Willd.) A.R. Sm. \& R.C. Moran. 14060.

Phanerophlebia juglandifolia (Humb. \& Bonpl. ex Willd.) J. Sm. 1335, 3544.

Phanerophlebia pumila (M. Martens \& Galeotti) Fée. 12428.

Phanerophlebia umbonata Underw. 16913, 17511, 17707, 21238, 21429, 22136, 24523, 24583.

Polystichum hartwegii (Klotzsch) Hieron. 22654.

Polystichum acrostichoides (Michx.) Schott. 21427, 22681. Polystichum distans E. Fourn. 2748, 3884, 7134, 13454.

Polystichum muricatum (L.) Fée. 14288.

Polystichum speciosissimum (A. Braun ex Kunze) Copel. 3295, 14310, 15730, 26214.

\section{Family Equisetaceae}

Equisetum giganteum L. 2402, 3332, 6223, 7957.

Equisetum hyemale L. 18772, 21856, 25588, 27410.

\section{Family Gleicheniaceae}

Gleichenella pectinata (Willd.) Ching. 5234, 7543.

Sticherus underwoodianus (Maxon) Nakai. 14239, 14305.

Family Hymenophyllaceae

Hymenophyllum crispum Kunth. 14306.

Hymenophyllum trapezoidale Liebm. 3268.

Trichomanes capillaceum L. 14307.

Trichomanes radicans Sw. 14165.

\section{Family Lindsaeaceae}

Odontosoria schlechtendalii (C. Presl) C. Chr. 10790, 11189.

\section{Family Lycopodiaceae}

Lycopodiella cernua (L.) Pic. Serm. 9017.

Phlegmariurus cuernavacensis (Underw. \& F.E. Lloyd) B. Øllg. 3221, 4053, 9966.

Phlegmariurus pringlei (Underw. \& F.E. Lloyd) B. Øllg. 4039, 8936.

Phlegmariurus reflexus (Lam.) B. Øllg. 3378.

Family Lygodiaceae

Lygodium venustum Sw. 7062, 7392, 10794, 11358.

\section{Family Marattiaceae}

Marattia weinmanniifolia Liebm. 14308.

\section{Family Nephrolepidaceae}

Nephrolepis cordifolia (L.) C. Presl. 18, 1641, 1805, 2588. Nephrolepis pectinata (Willd.) Schott. 11383, 11389.

Nephrolepis undulata (Afzel. ex Sw.) J. Sm. 9341, 9483, $11382,15491$.

\section{Family Onocleaceae}

Onocleopsis hintonii F. Ballard. 11211, 11211bis, 16314.

Onocleopsis hintonii F. Ballard. 3297 (TYPUS).

\section{Family Ophioglossaceae}

Botrychium schaffneri Underw. 8301.

Botrychium virginianum (L.) Sw. 1531, 1850, 22218, 22306, 24847, 25280.

Ophioglossum engelmannii Prantl. 4301, 25489.

\section{Family Osmundaceae}

Osmunda regalis L. 3660, 5394.

Family Plagiogyriaceae

Plagiogyria pectinata (Liebm.) Lellinger. 43, 2808, 3600, $6823,26045$.

\section{Family Polypodiaceae}

Alansmia cultrata (Bory ex Willd.) Moguel \& M. Kessler. 14280.

Campyloneurum angustifolium (Sw.) Fée. 3512, 4052, 6833, 10775, 15543, 15685, 22653, 24295, 24585, 24659, 25115.

Campyloneurum phyllitidis (L.) C. Presl. 16269.

Campyloneurum xalapense Fée. 11177, 26436, 26835.

Melpomene moniliformis (Lag. ex Sw.) A.R. Sm. \& R.C. Moran. 14294.

Melpomene pilosissima (M. Martens \& Galeotti) A.R. Sm. \& R.C. Moran. 14326.

Pecluma alfredii (Rosenst.) M.G. Price. 17208.

Pecluma plumula (Humb. \& Bonpl. ex Willd.) M.G. Price. 4161, 13178, 15478, 24528, 24790.

Phlebodium areolatum (Humb. \& Bonpl. ex Willd.) J. Sm. 25, 1529, 4096, 11161, 15493, 17178, 17446bis, 18052, 20885, 20889, 26301.

Pleopeltis angusta Humb. \& Bonpl. ex Willd. 11067, 15632.

Pleopeltis astrolepis (Liebm.) E. Fourn. 11870.

Pleopeltis fallacissima (Maxon) A.R. Sm. \& Tejero. 20138. Pleopeltis furfuracea (Schltdl. \& Cham.) A.R. Sm. \& Tejero. 27, 1254, 25039.

Pleopeltis madrensis (J. Sm.) A.R. Sm. \& Tejero. 6839, 26347, 26362.

Pleopeltis mexicana (Fée) Mickel \& Beitel. 31, 2344, 4163. Pleopeltis platylepis (Mett. ex Kuhn) A.R. Sm. \& Tejero. 7474, 13283, 26372.

Pleopeltis polylepis (Roem. ex Kuntze) T. Moore. 15652, 17371, 17511, 19044, 19107, 20136, 21301, 21800, 23471, $24315,24557,24883,26193,26237,26298,26447,26585$, 26620.

Pleopeltis polypodioides (L.) E.G. Andrews \& Windham. 20, 1253, 4162, 9435, 16931, 17954, 20820, 20919, 21218, 23065, 24121, 24449.

Pleopeltis rosei (Maxon) A.R. Sm. \& Tejero. 9940, 14498, 17174.

Pleopeltis thyssanolepis (A. Braun ex Klotzsch) E.G. Andrews \& Windham. 15495, 20876, 21128, 21129, 24652, 29745, 29762.

Polypodium eatonii Baker. 25062.

Polypodium fraternum Schltdl. \& Cham. 26824, 26828.

Polypodium guttatum Maxon. 17190, 19045, 19169, 19368, 19389, 20085, 22239, 24018, 24925, 25072, 26100.

Polypodium hartwegianum Hook. 1316, 14729, 15347, 
22134, 22154, 22251, 25087, 25289.

Polypodium martensii Mett. 13282, 23268, 26194, 26195, 26300.

Polypodium plesiosorum Kunze. 12249, 16920, 17515, 21346.

Polypodium pleurosorum Kunze ex Mett. 14232.

Polypodium remotum Desv. 11071, 17373, 24300, 24661, 26058.

Polypodium rhodopleuron Kunze. 9451, 11390.

Polypodium subpetiolatum Hook. 26, 1255, 1532, 15346, 19180, 21032, 21319, 22197, 22237, 22258.

Serpocaulon triseriale (Sw.) A.R. Sm. 11047.

\section{Family Psilotaceae}

Psilotum nudum (L.) P. Beauv. 3923.

\section{Family Pteridaceae}

Adiantopsis radiata (L.) Fée. 14703.

Adiantum capillus-veneris L. 16667.

Adiantum amplum C. Presl. 15821, 15883, 15906.

Adiantum andicola Liebm. 6841, 10174, 10299, 10733, 11130, 17092, 24893, 26257.

Adiantum braunii Mett. ex Kuhn. 9492, 9766, 9865, 10975. Adiantum capillus-veneris L. 15895, 16699, 17323, 17508, 18039, 19302, 21482, 25082, 25320.

Adiantum concinnum Humb. \& Bonpl. ex Willd. 1881, 4598, 6830, 7263, 7318, 9229, 10977, 11476, 15663.

Adiantum galeottianum Hook. 11375.

Adiantum lunulatum Burm. f. 8500, 9352, 9375, 10976.

Adiantum macrophyllum Sw. 10811.

Adiantum patens Willd. 38, 1683, 4751, 9493, 9767, 10816, 11354, 13491, 15669.

Adiantum poiretii Wikstr. 1674, 4861, 9864, 13525, 22687, 26253, 26424.

Adiantum shepherdii Hook. 1602, 4426, 4758, 9449, 9591, 11347, 13485, 15434.

Adiantum tenerum Sw. 9894, 10789, 10942.

Adiantum tetraphyllum Humb. \& Bonpl. ex Willd. 14678. Adiantum trapeziforme L. 7644, 8605, 10314, 10802, 10941, 12277, 16301.

Adiantum tricholepis Fée. 21043.

Anogramma leptophylla (L.) Link. 5350, 7335, 8613.

Argyrochosma delicatula (Maxon \& Weath.) Windham. 20925, 21025, 21597, 23260.

Argyrochosma incana (C. Presl) Windham. 21144, 22199.

Argyrochosma limitanea (Maxon) Windham. 16708, 16825, 19023, 19048, 19101, 20965, 20976.

Argyrochosma microphylla (Mett. ex Kuhn) Windham. 16510, 20186, 20970, 21505, 22373, 28943.

Aspidotis meifolia (D.C. Eaton) Pic. Serm. 17513, 17708, 22196, 22625, 24689, 25125.

Astrolepis cochisensis (Goodd.) D.M. Benham \& Windham. 16512, 16688, 16769, 16858, 17312, 18079, 19645, 19773, 23349, 28472, 28695, 28809.

Astrolepis crassifolia (Houlston \& T. Moore) D.M. Benham \& Windham. 17497bis, 22259.

Astrolepis integerrima (Hook.) D.M. Benham \& Windham. 16687, 16764, 19093, 21013, 23401, 23731, 27511, 28954,
28959.

Astrolepis laevis (M. Martens \& Galeotti) Mickel. 15400, 23228, 23433, 26126.

Astrolepis sinuata (Lag. ex Sw.) D.M. Benham \& Windham. 1966 (Type of Notholaena sinuata subsp. madrensis Hevly), 9724, 12687, 13265 (Type of Notholaena sinuata var. robusta Hevly), 16559, 16768, 17497, 19662, 19760, 20074, 20960, 23388, 28991.

Bommeria ehrenbergiana (Klotzsch) Underw. 21217, 22526, 23067.

Bommeria elegans (Davenp.) Ranker \& Haufler. 4740.

Bommeria pedata (Sw.) E. Fourn. 1431, 9209, 12210.

Cheilanthes lozanoi (Maxon) R.M. Tryon \& A.F. Tryon. 9170.

Cheilanthes brachypus (Kunze) Kunze. 9677, 15433.

Cheilanthes farinosa (Forssk.) Kaulf. 6836, 12363, 26127.

Cheilanthes horridula Maxon. 20927, 23367, 24259, 24275 , 27024.

Cheilanthes leucopoda Link. 20841, 21182.

Cheilanthes lozanoi (Maxon) R.M. Tryon \& A.F. Tryon. 4318, 6696, 9527, 12077.

Cheilanthes pyramidalis Fée. 9650, 13421, 15666.

Cheilanthes skinneri (Hook.) R.M. Tryon \& A.F. Tryon. 9476.

Cheilanthes spiculata Mickel. 6837 (TYPUS).

Cheiloplecton rigidum (Sw.) Fée. 12229, 13374, 15224, 20579.

Doryopteris pedata (L.) Fée. 14723.

Eriosorus flexuosus (Desv.) Copel. 14221, 14309.

Gaga angustifolia (Kunth) F.W. Li \& Windham. 32, 33, 39, 1500, 1684, 12190.

Gaga apiacea (Mickel) F.W. Li \& Windham. 23441.

Gaga decomposita (M. Martens \& Galeotti) F.W. Li \& Windham. 9491, 12209.

Gaga hintoniorum (M.G. Mendenh. \& G.L. Nesom) F.W. Li \& Windham. 18639, 18765 (TYPUS), 19315, 20157, 21042, 21513, 22350, 22695, 25694, 28136, 28587, 28936.

Gaga kaulfussii (Kunze) F.W. Li \& Windham. 16, 15697, 23712.

Gaga marginata (Kunth) F.W. Li \& Windham. 13497, 22087, 22121, 22153, 26131, 26356.

Gaga membranacea (Davenp.) F.W. Li \& Windham. 26207.

Hemionitis palmata L. 11052, 14721.

Hemionitis subcordata (D.C. Eaton ex Davenp.) Mickel. 8062, 12213.

Llavea cordifolia Lag. 16912, 17486bis, 17486, 20057, 20153, 21289, 22242, 24522

Mildella fallax (M. Martens \& Galeotti) G.L. Nesom. 9490, 22146, 23932, 24590.

Mildella intramarginalis (Kaulf. ex Link) Trevis. 15544, 23635.

Myriopteris aemula (Maxon) Grusz \& Windham. 24008.

Myriopteris alabamensis (Buckley) Grusz \& Windham. 16711, 20061, 20580, 20842, 20915, 21076, 21675.

Myriopteris allosuroides (Mett.) Grusz \& Windham. 29747.

Myriopteris aurea (Poir.) Grusz \& Windham. 30, 3309, 
9481, 17438, 18054, 19912, 21573, 21777, 21837, 22194, 22195, 22505, 23066, 23420, 23467, 23658, 23659, 23726, 24571, 26419, 29753, 29763.

Myriopteris lendigera (Cav.) J. Sm. 15542, 15696, 26238. Myriopteris longipila (Baker) Grusz \& Windham. 14378, 20853, 21018, 22857.

Myriopteris myriophylla (Desv.) Sm. 4165, 21111, 29746. Myriopteris notholaenoides (Desv.) Grusz \& Windham. 16826, 16922, 17324, 17487, 17496, 18092, 18385, 18700, 19042, 19453, 19768, 20092, 28987.

Myriopteris rufa Fée. 16857, 18704, 19031, 19049, 20076, 20855, 20866, 20975, 21562, 23649.

Myriopteris tomentosa (Link) Fée. 28938.

Myriopteris windhamii Grusz. 22608, 23339, 25762.

Notholaena aschenborniana Klotzsch. 16561, 19759, 21050 , 22607, 23657.

Notholaena aureolina Yatsk. \& Arbeláez. 6137.

Notholaena bryopoda Maxon. 16543, 18083, 19309, 19338, 19651, 20071.

Notholaena candida (M. Martens \& Galeotti) Hook. 6174, 7395, 9628, 17489, 19974, 20060, 20165, 20773, 20856, 22593, 24511.

Notholaena copelandii C.C. Hall. 21139.

Notholaena greggii (Mett. ex Kuhn) Maxon. 16870, 23374, 28473, 28643, 28906, 28940, 29478.

Notholaena neglecta Maxon. 21058, 22198.

Notholaena ochracea (Hook.) Yatsk. \& Arbeláez. 918, 4350.

Notholaena schaffneri (E. Fourn.) Underw. ex Davenp. 20924, 27862.

Notholaena standleyi Maxon. 19322, 27510, 28435.

Notholaena sulphurea (Cav.) J. Sm. 22257.

Pellaea ternifolia (Cav.) Link. 29786.

Pellaea atropurpurea (L.) Link. 17755, 17807, 18737, 19467, 20091, 21306, 21923, 24716.

Pellaea cordifolia (Sessé \& Moc.) A.R. Sm. 1539, 1831.

Pellaea intermedia Mett. ex Kuhn. 16700, 16824, 18100, 18299, 18691, 19094, 19095, 19687, 20078, 20863, 20972, 21028, 21241, 23389.

Pellaea notabilis Maxon. 20008.

Pellaea ovata (Desv.) Weath. 42, 1535, 7206, 7427, 11305, 21140, 21460, 23656.

Pellaea pringlei Davenp. 1537, 1538, 4227, 9475.

Pellaea sagittata (Cav.) Link. 17488.

Pellaea ternifolia (Cav.) Link. 15, 4061, 4234, 7425, 9223, 10490, 15497, 18057, 22152.

Pityrogramma calomelanos (L.) Link. 3, 2585, 3683, 7326, 14686, 15881, 16293, 16295.

Pityrogramma ebenea (L.) Proctor. 7434, 14190, 15487, 17153, 17460bis, 17512bis, 26336.

Pityrogramma trifoliata (L.) R.M. Tryon. 8464.

Pteris longifolia L. 24120.

Pteris cretica L. 5, 2804, 7424, 13447, 14206, 14287, 16926, 17154, 17504, 21638, 22122, 22683, 24593.

Pteris grandifolia L. 16025.

Pteris longifolia L. 3961, 4496, 24417.

Pteris muricella Fée. 14983.

Pteris orizabae M. Martens \& Galeotti. 7383.
Pteris propinqua J. Aghard. 6049.

Pteris pulchra Schltdl. \& Cham. 14208.

Pteris quadriaurita Retz. 14, 3508, 5907, 7324, 7414, 11663.

Scoliosorus ensiformis (Hook.) T. Moore. 11174, 14278.

Vittaria graminifolia Kaulf. 4238, 6162, 7369, 14231, 14272, 15496.

Vittaria lineata (L.) Sm. 7452.

\section{Family Selaginellaceae}

Selaginella apoda (L.) Spring. 25112, 25129.

Selaginella arizonica Maxon. 20084.

Selaginella gypsophila A.R. Sm. \& T. Reeves. 18609, 18633, 18751, 20160, 25647, 28115.

Selaginella hoffmannii Hieron. 8618, 15223.

Selaginella lepidophylla (Hook. \& Grev.) Spring. 7388, 19251, 19277, 19343, 20175, 20823, 21143, 21419, 23360, 25777, 28494, 29273, 29375, 29477.

Selaginella novoleonensis Hieron. 24122, 25037.

Selaginella pallescens (C. Presl) Spring. 1808, 3974, 4181, 9327, 9579, 10282, 20877, 20896, 22532, 22638, 23126, 24448, 24688, 25041, 26420.

Selaginella parishii Underw. 21112.

Selaginella peruviana (Milde) Hieron. 20938, 23716, 27778.

Selaginella pilifera A. Braun. 19104, 19234, 19265, 19281, 19335, 20004, 20059, 20396, 20571, 20824, 20852, 20932, 21041, 21100, 21138, 21165, 21202, 21393.

Selaginella ribae Valdespino. 19975.

Selaginella sartorii Hieron. 3453, 8423 (Type of Selaginella hintonii Weath.).

Selaginella schaffneri Hieron. 7391.

Selaginella sertata Spring. 11369.

Selaginella silvestris Aspl. 7350.

Selaginella tarda Mickel \& Beitel. 2491 (TYPUS).

Selaginella wrightii Hieron. 19755, 19783, 21141, 21183, 21403, 21441, 21783, 24553, 25669, 28729.

\section{Family Tectariaceae}

Tectaria mexicana (Fée) C.V. Morton. 10929.

Tectaria heracleifolia (Willd.) Underw. 3164, 5906, 6208, 7640, 14032, 15897, 16026, 16290, 17962.

Tectaria incisa Cav. 11021.

Tectaria mexicana (Fée) C.V. Morton. 10929bis, 11344, 12304, 16039, 16172.

\section{Family Thelypteridaceae}

Goniopteris imbricata (Liebm.) Á. Löve \& D. Löve. 14704.

Thelypteris ovata R.P. St. John. 22200.

Thelypteris augescens (Link) Munz \& I.M. Johnst. 16925.

Thelypteris concinna (Willd.) Ching. 1392.

Thelypteris cheilanthoides (Kunze) Proctor. 15376, 15377.

Thelypteris dentata (Forssk.) E.P. St. John. 16256.

Thelypteris grandis A.R. Sm. 9921, 10144, 10743, 13442, 14981, 15584.

Thelypteris ovata R.P. St. John. 19954, 25251.

Thelypteris patens (Sw.) Small. 10, 2803, 3241, 3766, 7430, 
14006.

Thelypteris pilosa (M. Martens \& Galeotti) Crawford. 3467, 15659.

Thelypteris pilosula (Klotzsch \& H. Karst. ex Mett.) R.M. Tryon. 13444.

Thelypteris puberula (Baker) C.V. Morton. 10077, 14194, 16284, 17509.

Thelypteris resinifera (Desv.) Proctor. 4966, 7205bis, 7205, 7323, 7368.

Thelypteris rudis (Kunze) Proctor. 13357, 15673.

\section{Family Woodsiaceae}

Woodsia mexicana Fée. 21005.

Woodsia mollis (Kaulf.) J. Sm. 1256, 1771, 1828, 4855, 9474, 13356, 15453, 22151, 22480.

Woodsia neomexicana Windham. 20081.

\section{Gymnosperms}

Family Cupressaceae

Cupressus arizonica Greene. 16760, 18948, 20311, 23319, 24371.

Cupressus lusitanica Mill. 647, 3246, 3247, 8801.

Juniperus deppeana Steud. 17816, 20198, 20498.

Juniperus flaccida Schltdl. 19899, 20197, 21194, 21547, 21623, 22763, 23911, 24579, 25910.

Juniperus monosperma (Engelm.) Sarg. 21983, 22047, 25355, 28931.

Juniperus monticola Martínez. 20201.

Juniperus pinchotii Sudw. 17854, 19103, 27176.

Juniperus saltillensis M.T. Hall. 18102, 19706.

Taxodium distichum (L.) Rich. 3232, 3881, 5209, 7144, 24168.

\section{Family Ephedraceae}

Ephedra antisyphilitica C.A. Mey. 25654, 25977, 28303.

Ephedra aspera Engelm. ex S. Watson. 16664, 18508, 19672 , 25705, 27424, 27458, 27501, 27553, 27665, 28500.

Ephedra compacta Rose. 16712, 16747, 25728, 25891.

Ephedra pedunculata Engelm. 17905.

\section{Family Pinaceae}

Abies durangensis Martínez. 18363.

Abies guatemalensis Rehder. 24682.

Abies hickelii Flous \& Gaussen. 26213.

Abies religiosa (Kunth) Schltdl. \& Cham. 3259.

Abies vejarii Martínez. 17274.

Picea chihuahuana Martínez. 22672, 23321, 24029, 24086.

Picea engelmannii J.P. Perry. 17972.

Pinus arizonica Engelm. 18753, 21649, 21803, 22052, 22701, 24058, 24461, 27009.

Pinus ayacahuite C. Ehrenb. ex Schltdl. 8802, 26282.

Pinus cembroides Zucc. 16795, 17112, 17492, 17768, 18150, 19370, 25348, 27008, 27723.

Pinus culminicola Andresen \& Beaman. 17275, 17920, 20200, 22423.

Pinus greggii Engelm. ex Parl. 17276, 18362, 19108, 22071
Pinus hartwegii Lindl. 17277, 17325, 17987, 19068, 19394, 26158.

Pinus leiophylla Schiede ex Schltdl. \& Cham. 3720, 5777, 5784, 7486, 7487.

Pinus maximinoi H.E. Moore. 5775, 10357.

Pinus montezumae Lamb. 3721, 5774, 5925, 6222, 19396, 22751, 24033.

Pinus oocarpa Schiede ex Schltdl. 3724, 7483, 10358.

Pinus patula Schiede ex Schltdl. \& Cham. 24025, 24034, 24038, 24529.

Pinus pinceana Gordon \& Glend. 21064, 22881, 29122.

Pinus pringlei Shaw. 5929.

Pinus pseudostrobus Lindl. 3722, 5773, 5776, 21643, 21644, 22050, 22051, 22629, 22633, 24028, 24035, 24041, 24043, 24326, 24457, 24568, 24958, 24959, 25186, 25187, 25327 , 26271, 26422.

Pinus remota (Little) D.K. Bailey \& Hawksw. 25975, 29464.

Pinus strobiformis Engelm. 17278, 19395, 19402.

Pinus teocote Schiede ex Schltdl. \& Cham. 3723, 5900, 7485, 21629, 21807, 22628, 24026, 24032, 24042, 24690, 24901, 25172.

Pseudotsuga menziesii (Mirb.) Franco. 17270, 17766, 18154, 18332, 18361, 18935, 19366, 22980.

\section{Family Taxaceae}

Taxus globosa Schltdl. 17506, 22520, 22650, 24848.

Family Zamiaceae

Dioon edule Lindl. 17712.

\section{Angiosperms}

Family Acanthaceae

Anisacanthus linearis (S.H. Hagen) Henr. \& E.J. Lott. 16577, 23186.

Anisacanthus quadrifidus (Vahl) Nees. 16837, 17824, 19602, 22523, 24283.

Aphelandra guerrerensis Wassh. 11199 (TYPUS).

Aphelandra hintonii Wassh. 16049 (TYPUS).

Aphelandra lineariloba Leonard. 3156, 7412 (TYPUS), 15794.

Aphelandra madrensis Lindau. 11648, 12675, 12881, 13623.

Aphelandra scabra (Vahl) Sm. 9903, 10919, 11477, 14691. Aphelandra verticillata Nees ex Hemsl. 2971, 13486.

Barleria oenotheroides Dum. Cours. 2857, 8738, 8764, 8782 , 9956, 11008, 11017, 11022, 11570, 11739, 12833, 12895, 13731, 14930, 16166.

Beloperone longibracteata Leonard. 2159 (TYPUS).

Buceragenia hirsuta Leonard. 5318 (TYPUS).

Carlowrightia arizonica A. Gray. 5533 (Type of Carlowrightia mucronata Leonard), 6129 (Type of Carlowrightia coyucana Leonard), 11216, 15886, 16195.

Carlowrightia hintonii T.F. Daniel. 11326 (TYPUS).

Carlowrightia linearifolia (Torr.) A. Gray. 27472.

Carlowrightia neesiana (Schauer ex Nees) T.F. Daniel. 19971. 
Carlowrightia pectinata Brandegee. 2672 (Type of Carlowrightia lanceolata Leonard), 2990.

Carlowrightia serpyllifolia (Torr.) A. Gray. 29242, 29309.

Chalarothyrsus amplexicaulis Lindau. 7384, 7624, 8867, 11270.

Dicliptera haenkeana Nees. 2498, 5450, 13309.

Dicliptera inaequalis Greenm. 3337 (Type of Dicliptera aquatica Leonard), 5602.

Dicliptera peduncularis Nees. 238.

Dicliptera resupinata (Vahl) A. Juss. 9840, 12619, 12865, 13400, 15774, 16233.

Dicliptera thlaspioides Nees. 3155, 5177.

Dyschoriste angustifolia (Hemsl.) Kuntze. 15387, 15601, 15688, 16253.

Dyschoriste greenmanii Kobuski. 24262, 25373.

Dyschoriste hirsutissima (Nees) Kuntze. 204, 2982, 9203, 11325, 11573, 12836, 12882, 13404, 14873, 16194, 24842. Dyschoriste linearis (Torr. \& A. Gray) Kuntze. 17140, 17529 , 18992, 19585, 19630, 21795, 23048, 23728, 23868, 24360, 24415, 24501, 25341, 25394, 25450, 25611, 25632, 25664, 25861, 25914, 27365, 27568, 28280, 28339, 29156, 29188. Dyschoriste ovata (Cav.) Kuntze. 1287, 1398, 4584, 11416 , 13071, 14412.

Dyschoriste poliodes Leonard \& Gentry. 17741, 17786.

Dyschoriste saltuensis Fernald. 11036, 14666.

Elytraria bromoides Oerst. 17956, 20352, 21541, 25243.

Elytraria imbricata (Vahl) Pers. 2616, 2962, 3135, 5111, 5669, 5762, 5953, 9791, 9812, 10995, 11578, 15321.

Henrya insularis Nees. 3410, 5756, 7611, 11217, 11779, 13606, 13639.

Holographis ehrenbergiana Nees. 22854.

Holographis hintonii (Leonard) T.F. Daniel. 5956 (TYPUS).

Holographis ilicifolia Brandegee. 28867.

Jacobinia mexicana Seem. 5190, 5715, 9679, 12620.

Justicia adenothyrsa (Lindau) T.F. Daniel. 11178.

Justicia pilosella (Nees) Hilsenb. 29876.

Justicia adenothyrsa (Lindau) T.F. Daniel. 11034.

Justicia aurea Schltdl. 12538, 16135.

Justicia carthaginensis Jacq. 8368, 10645, 10878.

Justicia caudata A. Gray. 26475.

Justicia clinopodia A. Gray ex Greenm. 1084, 4535, 8065.

Justicia fulvicoma Schltdl. \& Cham. 17619, 19980, 21226, 21463.

Justicia furcata Jacq. 13137.

Justicia hilsenbeckii T.F. Daniel. 9813.

Justicia hintoniorum G.L. Nesom. 20354 (TYPUS), 21216, 21416.

Justicia leonardii Wassh. 17746, 19285, 22859, 24143, 24280, 24624, 24971, 25244.

Justicia pilosella (Nees) Hilsenb. 20616, 21051, 25342, 25363, 27366, 28539, 29150.

Justicia pringlei B.L. Rob. 5122, 5245, 13244.

Justicia reflexiflora Rich. 10855, 10955, 11478, 12624, 15322.

Justicia salviiflora Kunth. 438, 3194, 7232, 11319, 11721, 12873, 15795, 16309.

Justicia spicigera Schltdl. 340, 3714, 12991.
Justicia turneri Hilsenb. 17686, 24201.

Justicia veracruzana T.F. Daniel. 14090.

Louteridium brevicalyx A.T. Richardson. 15825 (TYPUS).

Odontonema auriculatum (Rose) T.F. Daniel. 11215.

Odontonema callistachyum (Schltdl. \& Cham.) Kuntze. 11181.

Odontonema auriculatum (Rose) T.F. Daniel. 15812.

Odontonema callistachyum (Schltdl. \& Cham.) Kuntze. 12585.

Poikilacanthus capitatus (Leonard) Ramamoorthy. 1354 (TYPUS), 8075, 8100, 8598.

Pseuderanthemum alatum (Nees) Radlk. 16014.

Pseuderanthemum axillare Leonard. 3200 (TYPUS), 11330.

Pseuderanthemum praecox (Benth.) Leonard. 351, 8879, 11243, 11805, 13676, 14072, 14107, 22738.

Pseuderanthemum standleyi Leonard. 8865, 14159.

Ruellia oaxacana Leonard. 13770.

Ruellia alboviolacea Lindau. 11018.

Ruellia amoena Sessé \& Moc. 11727, 15826, 16287, 16310.

Ruellia blechum L. 2992, 5497, 13664, 17719.

Ruellia guerrerensis T.F. Daniel. 11296 (TYPUS).

Ruellia hookeriana (Nees) Hemsl. 8117, 10489, 10594.

Ruellia intermedia Leonard. 6443, 9074, 13004.

Ruellia inundata Kunth. 2659, 5685, 5871, 9820, 9837, 15323, 15986, 16193.

Ruellia lactea Cav. 1076, 4258.

Ruellia novogaliciana T.F. Daniel. 12931 (TYPUS).

Ruellia nudiflora (Engelm. \& A. Gray) Urb. 6275, 12015, 17621, 17953, 20390, 20550, 20593, 21537, 23612, 24411, 24641, 24784, 25386, 25512, 25650, 25868, 27514, 27738, 27820, 28855.

Ruellia oaxacana Leonard. 3922, 13757, 15831.

Ruellia parryi A. Gray. 23179, 25796, 25966, 29121.

Ruellia parva (Nees) Hemsl. 10195, 11756, 12611, 14116.

Ruellia petiolaris (Nees) T.F. Daniel. 11757.

Ruellia rubicaulis Cav. 9906, 11564, 14995.

Ruellia sarukhaniana Ramamoorthy. 12659 (TYPUS).

Ruellia spissa Leonard. 1068, 1231, 10523, 13039.

Ruellia tuberosa L. 5465bis.

Stenandrium manchoense T.F. Daniel. 10460 (TYPUS).

Stenandrium dulce (Cav.) Nees. 754, 4292 (Type of Stenandrium mexicanum Leonard), 6086, 7707, 8080, 8127, 18791, 18977, 19129, 20224.

Stenostephanus gracilis (Oerst.) T.F. Daniel. 10758.

Tetramerium glandulosum Oerst. 3375 (Type of Tetramerium hintonii Happ), 5724 (Type of Tetramerium hillii Happ), 15824, 15877.

Tetramerium glutinosum Lindau. 3589.

Tetramerium guerrerense T.F. Daniel. 11000 (TYPUS).

Tetramerium nervosum Nees. 437, 711, 2963, 2991, 5546, 8789, 9789, 9806, 11327, 11744, 12861, 12934, 15316, 17740, 24224, 25031, 28575.

Tetramerium rubrum G. Happ. 3767 (TYPUS), 10119, 11318, 13528, 14067.

Tetramerium tenuissimum Rose. 5494 (Type of Tetramerium leptocaule Happ), 5544, 5841, 6969, 12899, 16196, 16229. 
Thunbergia alata Bojer ex Sims. 4822, 5154.

Yeatesia mabryi Hilsenb. 24291, 24519, 24782.

Yeatesia platystegia (Torr.) Hilsenb. 21153.

\section{Family Actinidiaceae}

Saurauia serrata DC. 3597, 5322, 7358, 10700, 10748, 10812, 11342, 14737, 14958, 16257, 17493bis.

\section{Family Adoxaceae}

Sambucus nigra L. 3280, 3524, 3549, 5923, 9001, 24175.

Viburnum acutifolium Benth. 810, 885, 11951.

Viburnum elatum Benth. 422, 904, 3502, 9013.

Viburnum rafinesquianum Schult. 18683.

Viburnum stenocalyx (Oerst.) Hemsl. 10163, 10434, 14112.

Viburnum sulcatum (Oerst.) Hemsl. 26418.

\section{Family Aizoaceae}

Sesuvium portulacastrum L. 25994, 28754, 28799.

Sesuvium verrucosum Raf. 29300.

Trianthema portulacastrum L. 6191, 6276, 6428.

\section{Family Alismataceae}

Echinodorus andrieuxii (Hook. \& Arn.) Small. 6935, 6978, 15336.

Echinodorus tenellus (Mart. ex Schult. \& Schult. f.) Buchenau. 2667, 5817.

Sagittaria latifolia Willd. 5493.

\section{Family Alstroemeriaceae}

Bomarea acutifolia (Link \& Otto) Herb. 14222, 14300, 17223, 26565.

Bomarea edulis (Tussac) Herb. 902, 1536, 4634, 4803, 7087, $8111,14488,14601$.

\section{Family Altingiaceae}

Liquidambar styraciflua L. 22627, 24835, 26374bis.

\section{Family Amaranthaceae}

Achyranthes aspera L. 2932, 5816, 11725.

Alternanthera caracasana Kunth. 21366.

Amaranthus hybridus L. 2137, 3015, 13584, 15483, 23502, 27615.

Amaranthus palmeri S. Watson. 2482, 5532, 19507.

Amaranthus spinosus L. 2663, 5625.

Atriplex acanthocarpa (Torr.) S. Watson. 28753.

Atriplex canescens (Pursh) Nutt. 16834, 18369, 18777, 19420, 19487, 24760, 27555.

Atriplex muricata Humb. \& Bonpl. ex Willd. 20701.

Atriplex reptans I.M. Johnst. 23039, 27056, 27566, 28748.

Atriplex texana S. Watson. 25818.

Celosia argentea L. 5012.

Celosia moquini Guill. 12503, 16173.

Celosia nitida Vahl. 20547, 25689.

Celosia virgata Jacq. 25010.

Chamissoa altissima (Jacq.) Kunth. 10890.

Chenopodiastrum murale (L.) S. Fuentes, Uotila \& Borsch. 20220.

Chenopodium album L. 4023, 12979, 15482.
Chenopodium berlandieri Moq. 18440, 19498, 20806.

Chenopodium fremontii S. Watson. 16864, 19207.

Chenopodium leptophyllum Nutt. ex S. Watson. 25816.

Dysphania ambrosioides (L.) Mosyakin \& Clemants. 3857, 5675, 17608.

Dysphania graveolens (Willd.) Mosyakin \& Clemants. 4693, 4881, 6513, 7065, 19656, 19747, 19913, 23499, 23673, 29750.

Eurotia lanata (Pursh) Moq. 24771, 25356.

Froelichia arizonica Thornber ex Standl. 16861, 23574, 23711, 25902, 27075.

Gomphrena serrata L. 29792.

Gomphrena filaginoides M. Martens \& Galeotti. 26455.

Gomphrena pringlei Coult. \& Fisher. 1488.

Gomphrena serrata L. 1134, 1274, 1781, 1784bis, 2149, 4032, 4419, 5069, 5443, 5485, 6886, 15240, 19226, 21187 , 21461, 28126.

Guilleminea densa (Humb. \& Bonpl. ex Schult.) Moq. 29797.

Guilleminea densa (Humb. \& Bonpl. ex Schult.) Moq. 6197, 24726.

Hebanthe grandiflora (Hook.) Borsch \& Pedersen. 3249.

Iresine calea (Ibañez) Standl. 3153.

Iresine cassiniiformis Schauer. 446, 5892, 17748.

Iresine diffusa Humb. \& Bonpl. ex Willd. 145, 2225, 2412, 2602, 15307, 15607, 15758, 17523bis, 21390, 26264, 26368, 26427, 26431, 26595, 26734, 26820, 26826.

Iresine hartmanii Uline. 2927, 3129.

Iresine heterophylla Standl. 19225, 20022, 20587, 21660, 28165.

Iresine interrupta Benth. 292, 3006, 12904.

Iresine jaliscana Uline \& W.L. Bray. 2542, 2988.

Iresine leptoclada (Hook. f.) Henr. 16849, 20830, 23718.

Iresine orientalis G.L. Nesom. 18124, 19286, 22757, 25022 , 25209.

Iresine paniculata L. 13602, 13803.

Lagrezia monosperma (Rose) Standl. 11689, 16200.

Salsola kali L. 19509, 19677.

Suaeda jacoensis I.M. Johnst. 27879.

Suaeda mexicana (Standl.) Standl. 25883.

Suaeda nigra (Raf.) J.F. Macbr. 16801, 25815, 27053, 27564 , 28677, 28678.

Suaeda palmeri (Standl.) Standl. 23552, 25722, 28801.

Tidestromia gemmata I.M. Johnst. 29569.

Tidestromia suffruticosa (Torr.) Standl. 27421, 29368.

Tidestromia lanuginosa (Nutt.) Standl. 16685, 16868, 20615, 23579, 24745, 28812, 28834.

Tidestromia suffruticosa (Torr.) Standl. 16558, 25737, 28397, 29301.

\section{Family Amaryllidaceae}

Allium glandulosum Link \& Otto. 1595, 4487, 4624, 4639, 9738, 12350, 23248, 23461, 23823, 24609.

Allium hintoniorum B.L. Turner. 18242, 21444 (TYPUS), 23057, 23216.

Allium kunthii G. Don. 16522, 20725, 21524, 22344, 23603, 23704, 23744, 23844, 25663, 27779.

Crinum americanum L. 1019. 
Hymenocallis harrisiana Herb. 707, 897, 4248.

Hymenocallis littoralis (Jacq.) Salisb. 938, 1080, 1123.

Hymenocallis proterantha Bauml. 15972 (TYPUS).

Jaimehintonia gypsophila B.L. Turner. 20560, 22461, 23695, 24005, 25724.

Nothoscordum bivalve (L.) Britton. 504, 857, 16927, 17536, 20264, 23084, 23725, 23818, 24700, 27191.

Sprekelia formosissima (L.) Herb. 29835.

Sprekelia formosissima (L.) Herb. 516, 893, 3930, 11882, 14193.

Zephyranthes chlorosolen (Herb.) D. Dietr. 28332.

Zephyranthes drummondii D. Don. 24413.

Zephyranthes lindleyana Herb. 943, 4175.

Zephyranthes minuta (Kunth) D. Dietr. 4037, 4040, 4168.

Zephyranthes morrisclintii Traub \& T.M. Howard. 18190,

18942, 20389, 20914, 20940, 21921, 22816.

\section{Family Anacampserotaceae}

Grahamia coahuilensis (S. Watson) G.D. Rowley. 20819, 27632, 29128.

Grahamia frutescens (A. Gray) G.D. Rowley. 20816, 23045, 25675, 25824.

\section{Family Anacardiaceae}

Amphipterygium adstringens (Schltdl.) Standl. 926, 4278, 4812, 6111, 13295.

Astronium graveolens Jacq. 14010.

Comocladia macrophylla (Hook. \& Arn.) Riley. 443, 3128, 3640, 5985, 10003.

Cyrtocarpa procera Kunth. 3956, 5792, 6019.

Mangifera indica L. 337, 3013.

Pistacia mexicana Kunth. 10258, 11983, 12135, 14075, 14262, 17288, 22163, 24547.

Pseudosmodingium perniciosum (Kunth) Engl. 1993, 5198, 6868, 6930, 6946, 7034, 9794bis, 15300.

Rhus muelleri Standl. \& F.A. Barkley. 29889.

Rhus andrieuxii Engelm. 16808.

Rhus aromatica Aiton. 18801, 19332, 21814, 22643, 23900, 24072, 25095, 25181, 27841.

Rhus lanceolata (A. Gray) Britton. 21234, 24606.

Rhus microphylla Engelm. 16560, 16730, 18392, 18660, 18708, 19381, 21896, 21968, 24467, 25346, 25704, 25712, 27549.

Rhus muelleri Standl. \& F.A. Barkley. 18082, 19208, 21068, 24404, 24937, 24965, 25584, 28726.

Rhus pachyrrhachis Hemsl. 17473, 21747, 22158, 22214, 23602, 24572, 25096.

Rhus terebinthifolia Schltdl. \& Cham. 3105, 5026, 5027, 13526, 14012.

Rhus trilobata Nutt. 2817, 2955, 4257, 7214, 7343, 8781, 14068, 18713.

Rhus virens Lindh. ex A. Gray. 17448, 19228, 21753, 23838, 23889, 24984, 25707, 25780, 28061, 28490, 29292.

Schinus molle L. 3125, 24831.

Spondias mombin L. 6599.

Spondias purpurea L. 3217, 3316, 5676, 10037.

Toxicodendron radicans (L.) Kuntze. 517, 630, 2821, 3898, 9488, 10492, 10551, 11835, 13151, 21579, 24124, 24127.

\section{Family Annonaceae}

Annona cherimola Mill. 473, 3703, 7637, 10772.

Annona globiflora Schltdl. 24787, 25141, 25253.

Annona longiflora S. Watson. 998, 3926, 4060, 4288, 4676, 5927, 6051, 6201, 7632, 7633, 7641, 7725, 7776, 7779, 7827, 7836, 7927.

Annona macroprophyllata Donn. Sm. 4281, 5802, 6718, 7863, 7865.

Annona reticulata L. 3824, 4831, 5578, 6020, 6023, 7054, 7301, 7917, 10277.

Cymbopetalum gracile R.E. Fr. 10257 (TYPUS).

Cymbopetalum hintonii Lundell. 13788 (TYPUS), 15893.

Desmopsis mexicana R.E. Fr. 15823, 15836 (TYPUS).

Oxandra lanceolata (Sw.) Baill. 16177.

Sapranthus violaceus (Dunal) Saff. 4564, 9102, 10318, 10340, 10343, 10344.

\section{Family Apiaceae}

Angelica nelsonii J.M. Coult. \& Rose. 529, 4191, 8254.

Angelica polycarpa J.M. Coult. \& Rose. 11128.

Apium graveolens L. 17137.

Arracacia aegopodioides (Kunth) J.M. Coult. \& Rose. 17379 , 20986, 22149, 22204, 22843, 22932, 22951, 22970.

Arracacia atropurpurea (Lehm.) Benth. \& Hook. f. ex Hemsl. 535, 871, 1922, 2687, 3274, 4643, 6762, 6821, 7211, $7219,7352,7416,7934,8904,10165,14410,26635,26643$, 26644, 26842.

Arracacia hintonii Constance \& Affolter. 18410, 18838, 18887 (TYPUS), 19124, 19135, 19159, 22472.

Arracacia longipedunculata J.M. Coult. \& Rose. 1142, 2121, 4232, 4445, 7939.

Arracacia rigida J.M. Coult. \& Rose. 849.

Arracacia schneideri Mathias \& Constance. 17045, 17337, 18829, 18862, 18890, 19120, 19155, 20141, 22671, 22753, 24023, 24604, 24701, 25336.

Arracacia ternata Mathias \& Constance. 17391, 19034, 20287, 20457, 23209, 23254, 23312, 23438, $23490,24074$.

Arracacia tolucensis (Kunth) Hemsl. 18271, 18430, 18809, 18847, 18859, 18886, 20306, 22299, 23965, 24312.

Berula erecta (Huds.) Coville. 654, 4054, 4095, 17852, 18041.

Bowlesia flabilis J.F. Macbr. 1560, 4879.

Cicuta maculata L. 24406.

Coulterophytum laxum B.L. Rob. 12360.

Cyclospermum leptophyllum (Pers.) Sprague ex Britton \& P. Wilson. 594, 3458, 3871, 5807, 7627, 20613, 22510, 23235.

Daucus carota L. 24146, 24397, 25408.

Daucus montanus Humb. \& Bonpl. ex Spreng. 821, 3870, 12373, 15267, 15520, 22148, 22230, 24538, 24565.

Donnellsmithia biennis (J.M. Coult. \& Rose) Mathias \& Constance. 1795, 2329, 4851, 4860, 7967, 8367, 8797, 17443bis.

Donnellsmithia hintonii Mathias \& Constance. 8463 (TYPUS).

Donnellsmithia juncea (Spreng.) Mathias \& Constance. 1081, 1238, 4253, 4422, 4690, 13960, 28946.

Donnellsmithia mexicana (B.L. Rob.) Mathias \& Constance. 
1848, 4649, 7136, 8657, 15600.

Donnellsmithia serrata (J.M. Coult \& Rose) Mathias \& Constance. 14324.

Donnellsmithia ternata (S. Watson) Mathias \& Constance. 18764, 18998, 19464, 20277, 20305, 20425, 22060, 22069, 22207, 22276, 22392, 23305, 25385, 27219.

Enantiophylla heydeana J.M. Coult. \& Rose. 915, 1013, 1090, 6562, 7909, 7949.

Eryngium pectinatum C. Presl ex DC. 29806.

Eryngium alternatum J.M. Coult. \& Rose. 26163, 26562.

Eryngium beecheyanum Hook. \& Arn. 190, 2194, 2774, 2941, 15674.

Eryngium bonplandii F. Delaroche. 1706, 8377, 15380.

Eryngium crassisquamosum Hemsl. 8679.

Eryngium cymosum F. Delaroche. 7333.

Eryngium galeottii Hemsl. 2759, 6842.

Eryngium ghiesbreghtii Decne. 674, 1146, 1353, 1542, 15044, 15612, 17155.

Eryngium gracile F. Delaroche. 2014, 12188, 14762, 15381, 26072, 26379, 26752.

Eryngium gramineum F. Delaroche. 18913, 19113, 22068, 22096, 23159, 23280, 25403.

Eryngium heterophyllum Engelm. 18562, 25398.

Eryngium longifolium Cav. 7346, 14883, 24338.

Eryngium monocephalum Cav. 7711.

Eryngium pectinatum C. Presl ex DC. 3270.

Eryngium proteiflorum F. Delaroche. 8338.

Eryngium serratum Cav. 18282, 21274, 22231, 23210, 25417, 29024, 29205.

Eryngium subacaule Cav. 1041.

Eryngium venustum Bartlett \& Constance. 19174, 19217, 20362, 21167, 21333, 22442, 22546, 23794, 23912, 24801.

Foeniculum vulgare Mill. 3872, 5355, 19482.

Mathiasella bupleuroides Constance \& C.L. Hitchc. 24327.

Mathiasella bupleuroides Constance \& C.L. Hitchc. 22234, 22393, 22829, 22960, 23249, 25269.

Micropleura renifolia Lag. 901, 1022, 4242, 13881, 14384. Neogoezia gracilipes (Hemsl.) Hemsl. 2029, 2158, 2391, 4971.

Neogoezia minor Hemsl. 26184, 26642, 26669.

Neogoezia planipetala (Hemsl.) Hemsl. 12340.

Osmorhiza geohintonii B.L. Turner. 22116 (TYPUS), 27390.

Osmorhiza mexicana Griseb. 1308, 4899, 8324, 21345, 21608, 22388, 22578, 23154, 25272, 27230, 29034.

Ottoa oenanthoides Kunth. 14267, 26217, 26390, 26551, 26589.

Prionosciadium cuneatum J.M. Coult. \& Rose. 8040, 10293.

Prionosciadium diversifolium Rose. 1262, 1919, 7886, 7921, 8093, 10547.

Prionosciadium humile Rose. 22181, 22602, 24337.

Prionosciadium nelsonii J.M. Coult. \& Rose. 834, 4184, 7891, 7901, 7907, 7928, 7942, 10512, 14382, 15007, 26281.

Prionosciadium thapsoides (DC.) Mathias. 4108, 7962, 8074.

Prionosciadium watsoni J.M. Coult. \& Rose. 26414.

Rhodosciadium diffusum (J.M. Coult. \& Rose) Mathias \&
Constance. 1662, 8099, 8138.

Rhodosciadium glaucum J.M. Coult. \& Rose. 2215.

Rhodosciadium pringlei S. Watson. 1511.

Rhodosciadium purpureum (Rose) Mathias \& Constance. 13922.

Rhodosciadium tolucense (Kunth) Mathias. 4608.

Sanicula liberta Cham. \& Schltdl. 21245, 21625, 22139, 22308, 22894, 22930, 23155, 23238, 24075, 24137, 24601, 24894, 25273.

Spananthe paniculata Jacq. 1752, 2264, 2429, 4674, 7516.

Tauschia decumbens (Benth.) J.M. Coult. \& Rose. 583.

Tauschia filiformis (J.M. Coult. \& Rose) J.M. Coult. \& Rose. 26542, 26575, 26796.

Tauschia hintoniorum Constance \& Affolter. 18200, 18426 (TYPUS), 18803, 18806, 18842, 18861, 18891, 18903.

Tauschia infernicola Constance \& Affolter. 20298, 21810, 23087.

Tauschia johnstoniana Mathias \& Constance. 18940, 18957, 20151, 20234, 20290, 20971, 28327.

Tauschia madrensis Rose. 17018, 17330, 18206, 25334.

Tauschia nudicaulis Schltdl. 606, 679.

Tauschia vaginata (J.M. Coult. \& Rose) J.M. Coult. \& Rose. 26524.

Torilis arvensis (Huds.) Link. 24424.

\section{Family Apocynaceae}

Allotoonia woodsoniana (Monach.) J.F. Morales \& J.K. Williams. 7589.

Allotoonia woodsonianus (Monach.) J.F. Morales \& J.K. Williams. 15325 (TYPUS).

Apocynum cannabinum L. 18485, 24340.

Apocynum x floribundum Greene. 17900.

Asclepias angustifolia Schweigg. 328, 1402, 3541, 5759, 24829, 24846.

Asclepias asperula (Decne.) Woodson. 20300.

Asclepias auriculata Kunth. 1396, 1629, 1679, 9552, 10716, 15590.

Asclepias brachystephana Engelm. ex Torr. 4872, 14216, 25347, 27656.

Asclepias curassavica L. 288, 426, 1668, 5657, 7133, 8987, 9480, 10007, 10981, 15398bis, 17829, 19936.

Asclepias elata Benth. 8188.

Asclepias emoryi (Greene) Vail ex Small. 18423.

Asclepias fascicularis Decne. 25411.

Asclepias glaucescens Kunth. 349, 3233, 5733, 12966 , 15789.

Asclepias jaliscana B.L. Rob. 1082, 1239, 9204, 14394.

Asclepias linaria Cav. 671, 7432, 7930, 11841, 16549, 16658, 19339, 19604, 19921, 20350, 20928, 22011, 22714, 24527, 25753.

Asclepias lynchiana Fishbein. 1472, 4599, 8194, 8547.

Asclepias notha W.D. Stevens. 3689, 4399.

Asclepias oenotheroides Schltdl. \& Cham. 16714, 16799, 25365, 27339.

Asclepias otarioides E. Fourn. 18758, 20460.

Asclepias ovata M. Martens \& Galeotti. 1357, 1430, 7994, 7998, 10635.

Asclepias pellucida E. Fourn. 7713, 8838, 10157, 11113. 
Asclepias pringlei (Greenm.) Woodson. 4325, 6776.

Asclepias pseudorubricaulis Woodson. 27211.

Asclepias rosea Kunth. 3409, 3588, 7180, 7554, 7692, 10107, 14197.

Asclepias scaposa Vail. 19704, 21893.

Asclepias similis Hemsl. 400, 598, 4237, 5772, 17805.

Asclepias subverticillata (A. Gray) Vail. 17135, 17858, 20605, 27584, 27648.

Asclepias tuberosa L. 19558, 21242, 22889, 22903, 24194, 24318.

Asclepias virletii E. Fourn. 18172, 21854, 21887, 21906, 27630, 27640.

Blepharodon mucronatum (Schltdl.) Decne. 1358, 7074, 14692.

Cascabela ovata (Cav.) Lippold. 4150, 8026, 9176, 9190, 14251.

Cascabela thevetia (L.) Lippold. 5716, 5732, 7579, 9178, 10532, 11153, 11975, 12018.

Cascabela thevetioides (Kunth) Lippold. 358, 830, 3418, 7961.

Catharanthus roseus (L.) G. Don. 4280.

Cynanchum foetidum (Cav.) Kunth. 1763, 1856, 2226, 4849, 8372, 14491, 15149.

Cynanchum ligulatum (Benth.) Woodson. 4484, 4737, 5564, 6429, 7146, 9373, 13092.

Dictyanthus asper (Mill.) W.D. Stevens. 1476, 7476bis, 8189.

Dictyanthus parviflorus Hemsl. 1690, 4328, 4471, 8008.

Dictyanthus pavonii Decne. 1282, 1498, 4324, 4357, 4590, 7905, 8014, 8201, 8223, 10592, 13275, 14481, 15061.

Echites yucatanensis Millsp. 10356.

Fernaldia asperoglottis Woodson. 3372, 7239, 7261, 15329.

Funastrum bilobum (Hook. \& Arn.) J.F. Macbr. 11610, 12616, 16249.

Funastrum clausum (Jacq.) Schltr. 6452, 9370, 10607, 14602, 15833.

Funastrum cynanchoides (Decne.) Schltr. 8743.

Funastrum elegans (Decne.) Schltr. 4936, 7225, 15075, 29709.

Funastrum pannosum (Decne.) Schltr. 1191, 6470, 9175, 10528, 13077.

Funastrum torreyi (A. Gray) Schltr. 19008, 24473, 25774.

Gonolobus barbatus Kunth. 6293, 8031, 8213, 10379, 10608, 14556.

Gonolobus erianthus Decne. 7311, 8453, 16919.

Gonolobus fraternus Schltdl. 13895, 14357.

Gonolobus jaliscensis B.L. Rob. \& Greenm. 988, 1122, 1293, 3819, 4375, 4421, 5076, 7023.

Gonolobus megalocarpus Paul G. Wilson. 454, 1381, 5899, 7141 (TYPUS), 7164.

Gonolobus pectinatus Brandegee. 1150, 1199, 9152, 13079.

Gonolobus sororius A. Gray. 4155.

Gonolobus uniflorus Kunth. 826, 3817, 17209.

Haplophyton cimicidum A. DC. 4359, 4465, 6921.

Laubertia contorta (M. Martens \& Galeotti) Woodson. 1156, 4724, 5009, 7919, 9183, 10544.

Macroscepis diademata (Ker Gawl.) W.D. Stevens. 4570,
7264, 11424, 14591.

Macroscepis pleistantha Donn. Sm. 8178, 8496, 9627.

Mandevilla torosa (Jacq.) Woodson. 24709.

Mandevilla foliosa (Müll. Arg.) Hemsl. 6379, 11514, 13980.

Mandevilla holosericea (Sessé \& Moc.) J.K. Williams. 1295, 4307, 5274, 7176.

Mandevilla hypoleuca (Benth.) Pichon. 1137, 1224, 6251, 7716, 8115, 13069, 22990.

Mandevilla lanuginosa (M. Martens \& Galeotti) Pichon. 20372, 23009, 24289, 25521, 27371, 27748, 27753, 28548.

Mandevilla macrosiphon (Torr.) Pichon. 23185.

Mandevilla subsagittata (Ruiz \& Pav.) Woodson. 10805, 10833, 16153.

Mandevilla subsessilis (A. DC.) Woodson. 9319, 13059.

Mandevilla torosa (Jacq.) Woodson. 16526, 16812, 17559 , 17948, 19619, 20255, 22946, 24052, 25366.

Marsdenia tubularis L.O. Williams. 1129 (TYPUS).

Marsdenia bourgaeana (Baill.) W. Rothe. 3972, 7900, 9082.

Marsdenia coulteri Hemsl. 17939.

Marsdenia lanata (Paul G. Wilson) W.D. Stevens. 1158, 3953, 5385, 6265, 7166, 7602 (TYPUS), 9150, 9526.

Marsdenia mexicana Decne. 559, 759, 4066, 7438, 7929, 10137, 13827.

Marsdenia pringlei S. Watson. 18119, 24126.

Marsdenia propinqua Hemsl. 6274.

Marsdenia trivirgulata Bartlett. 7003, 10979.

Marsdenia zimapanica Hemsl. 931, 3983, 13853, 13861.

Matelea caudata (A. Gray) Woodson. 1468, 4557, 5545, 5795, 6016.

Matelea congesta (Decne.) Woodson. 4677, 6563, 11394.

Matelea crenata (Vail) Woodson. 8053, 8135, 9249, 10498, 13161.

Matelea chrysantha (Greenm.) Woodson. 1094, 8439, 15488, 15500, 17228.

Matelea gonoloboides (B.L. Rob. \& Greenm.) Woodson. 5060 .

Matelea pilosa (Benth.) Woodson. 1198, 1230, 4410, 6703, 8094, 9105, 15079, 17618, 24152, 24181, 24851.

Matelea reticulata (Engelm. ex A. Gray) Woodson. 24296.

Metastelma barbigerum Scheele. 17660, 19732, 20622 , 24186, 24710, 27364, 28489.

Metastelma lanceolatum Schltr. 7310.

Metastelma palmeri Liede \& Meve. 17935 (TYPUS), S. Watson. 16830, 17776, 24258.

Metastelma pringlei A. Gray. 16528, 19616, 19712, 20414, 21088, 23343, 25477.

Metastelma schaffneri A. Gray. 1832, 4715, 7030.

Nerium oleander L. 265, 6267, 7785.

Orthosia angustifolia Turcz. 18659, 24347.

Orthosia pubescens (Greenm.) Liede \& Meve. 1106, 2680, 4073, 4449, 5082.

Pherotrichis villosa Meisn. 1406, 4482, 14396, 15128.

Plumeria rubra L. 375, 519, 520, 727, 3831, 3949, 3985, 3986, 3987, 8151, 10032, 10439.

Polystemma guatemalense (Schltr.) W.D. Stevens. 1450 (Type of Matelea hintoniana Woodson), 4722, 6322, 8097, 
12005, 13084, 14530, 15957.

Polystemma viridiflorum Decne. 6459, 8017, 8522.

Prestonia mexicana A. DC. 4508, 5051, 5214, 11365, 14492.

Prosthecidiscus guatemalensis Donn. Sm. 7290.

Rauvolfia tetraphylla L. 724, 3582, 3826, 5799, 6018, 10325, 10874, 12028, 13814.

Suberogerens cyclophylla (Standl.) Morillo. 4361.

Tabernaemontana donnell-smithii Rose. 10043, 10186.

Tabernaemontana litoralis Kunth. 4569.

Tabernaemontana tomentosa (Greenm.) A.O. Simões \& M.E. Endress. 441, 3582bis, 7625, 10111, 12982, 15903.

Tabernaemontana amygdalifolia Jacq. 3761, 5674, 7260, 10324, 10993, 14117.

Tabernaemontana odontadeniiflora A.O. Simões \& M.E. Endress. 722, 5983.

Thenardia floribunda Kunth. 2150, 8204, 12253, 12481, 15989.

Tonduzia longifolia (A. DC.) Markgr. 3968, 8088, 11391.

Trichosacme lanata Zucc. 23755.

Vinca major L. 4890, 12781, 19404.

\section{Family Aquifoliaceae}

Ilex brandegeana Loes. 320, 336, 3298, 3529, 3598, 3603, 7207, 8969, 9570, 12701, 14224.

Ilex condensata Turcz. 24820.

Ilex discolor Hemsl. 532, 541, 3497, 3698.

Ilex rubra $\mathrm{S}$. Watson. 22544.

\section{Family Araceae}

Anthurium schlechtendalii Kunth. 10098, 10125.

Arisaema dracontium (L.) Schott. 3484, 18272, 22531, 22652, 22739, 23983.

Arisaema macrospathum Benth. 4042, 4176, 6775, 11985.

Lemna minuta Kunth. 18038.

Syngonium neglectum Schott. 3808, 3984.

Xanthosoma mexicanum Liebm. 26374.

Xanthosoma robustum Schott. 7821.

Xanthosoma sagittifolium (L.) Schott. 16047.

\section{Family Araliaceae}

Aralia humilis Cav. 1789, 4430, 5141, 9605.

Dendropanax arboreus (L.) Decne. \& Planch. 263, 362, 3326, 3392, 3673, 3713, 3806, 7550, 11733, 15412, 15814, 15817.

Hydrocotyle mexicana Schltdl. \& Cham. 9893, 26428.

Hydrocotyle ranunculoides L. f. 4196.

Hydrocotyle verticillata Thunb. 4156, 25236, 25821.

Oreopanax echinops (Cham. \& Schltdl.) Decne. \& Planch. 12776.

Oreopanax peltatus Linden. 2422bis, 3204, 3907, 4035, 7040, 7186, 7440, 13561.

Oreopanax xalapensis (Kunth) Decne. \& Planch. 1103, 5059, 5279, 14084, 14966.

\section{Family Arecaceae}

Brahea dulcis (Kunth) Mart. 19984, 21888, 21889, 24545.

Brahea moorei L.H. Bailey ex H.E. Moore. 25033.
Brahea pimo Becc. 5250, 10430.

Chamaedorea pochutlensis Liebm. 15984.

Chamaedorea radicalis Mart. 25114.

Sabal mexicana Mart. 4122, 25261, 25315.

\section{Family Aristolochiaceae}

Aristolochia lassa I.M. Johnst. 21939.

Aristolochia brevipes Benth. 4302, 4309, 4707.

Aristolochia bullata Pfeifer. 14569 (TYPUS).

Aristolochia cardiantha Pfeifer. 4354, 9096 (TYPUS).

Aristolochia conversiae Pfeifer. 8110.

Aristolochia foetida Kunth. 6295.

Aristolochia glossa Pfeifer. 15151 (TYPUS).

Aristolochia mycteria Pfeifer. 3141, 13530.

Aristolochia odoratissima L. 16147.

Aristolochia orbicularis Duch. 8467.

Aristolochia secunda Pfeifer. 22853, 25811.

Aristolochia taliscana Hook. \& Arn. 3565, 4310, 5565, 11605, 12623, 15816, 16167.

Aristolochia wrightii Seem. 24750.

\section{Family Asparagaceae}

Agave americana L. 20629, 20632, 24549, 24580, 25144.

Agave angustiarum Trel. 7561.

Agave asperrima Jacobi. 19355.

Agave atrovirens Karw. ex Salm-Dyck. 18265, 18429, 18444, 18945, 19401.

Agave celsii Hook. 22280, 24302.

Agave funkiana K. Koch \& C.D. Bouché. 18722.

Agave gentryi B. Ullrich. 20364, 22177, 22262.

Agave gypsophila Gentry. 15764.

Agave inaequidens K. Koch. 7512.

Agave lophantha Schiede. 17809.

Agave striata Zucc. 18986, 19635, 24542, 24660, 25340.

Beschorneria septentrionalis García-Mend. 25267.

Bessera elegans Schult. f. 939, 1083, 1516, 1598, 1840, $6505,9180,9351,9404,9704,10543,12035,12185,13072$, 13902, 14353, 15547.

Dasylirion berlandieri S. Watson. 17113, 17793, 18755, 22573, 24308, 24421.

Dasylirion quadrangulatum S. Watson. 23108, 27338.

Echeandia atoyacana Cruden. 6321, 14526.

Echeandia echeandioides (Schltdl.) Cruden. 2946.

Echeandia hintonii Cruden. 10763.

Echeandia hirticaulis Cruden. 1263, 1852, 9596, 13146.

Echeandia mexicana Cruden. 29723.

Echeandia nana (Baker) Cruden. 18018, 29751.

Echeandia coalcomanensis Cruden. 15034 (TYPUS).

Echeandia durangensis (Greenm.) Cruden. 25410, 25452, 27187.

Echeandia echeandioides (Schltdl.) Cruden. 10604.

Echeandia flavescens (Schult. \& Schult. f.) Cruden. 1344, 9332, 14095, 17364, 17554, 19009, 19160, 20451, 20494, 25607, 27715.

Echeandia flexuosa Greenm. 3243.

Echeandia formosa (Weath.) Cruden. 26761.

Echeandia mexicana Cruden. 21174, 21281, 24674, 24715.

Echeandia paniculata Rose. 17238, 23213. 
Echeandia reflexa (Cav.) Rose. 2032, 8387, 8863, 13355, 15255, 15560, 23307, 26325.

Echeandia skinneri (Baker) Cruden. 9962, 25458.

Echeandia tenuis (Weath.) Cruden. 8895, 8986, 11263.

Furcraea guerrerensis Matuda. 4660.

Hemiphylacus hintoniorum L. Hern. 19431, 21186 (TYPUS), 22094, 22603.

Hemiphylacus latifolius S. Watson. 16665.

Hesperaloe funifera (K. Koch) Trel. 24263.

Maianthemum paniculatum (M. Martens \& Galeotti)

LaFrankie. 26177, 26599.

Maianthemum scilloideum (M. Martens \& Galeotti) LaFrankie. 4520, 5079, 7691, 7953, 14290, 14407, 20994.

Maianthemum stellatum (L.) Link. 17577, 18860, 27542.

Manfreda jaliscana Rose. 15786.

Manfreda longiflora (Rose) Verh.-Will. 27755.

Manfreda planifolia (S. Watson) Rose. 14317.

Manfreda potosina (B.L. Rob. \& Greenm.) Rose. 27200.

Manfreda pringlei Rose. 13927.

Manfreda pubescens (Regel \& Ortgies) Verh.-Will. ex Pina. 1050, 1220, 1245.

Manfreda scabra (Ortega) McVaugh. 2789, 9201, 19892, 20244, 21099, 24765, 25331, 25424.

Manfreda variegata (Jacobi) Rose. 22924, 24407.

Milla biflora Cav. 1189, 1235, 6500, 9329, 12147, 13129, 15133.

Milla delicata H.E. Moore. 9920 (TYPUS).

Milla mortoniana H.E. Moore. 9725 (TYPUS).

Nolina azureogladiata D. Donati. 29252 (TYPUS collected by D. Donati).

Nolina cespitifera Trel. 18257, 21059, 28584.

Nolina hibernica Hochstätter \& D. Donati. 29065 (TYPUS collected by D. Donati).

Nolina nelsonii Rose. 22837.

Polianthes geminiflora (Lex.) Rose. 1412, 4456, 4527, 12165, 13921, 15042.

Yucca carnerosana (Trel.) McKelvey. 19658, 20309.

Yucca decipiens Trel. 21873.

Yucca filifera Chabaud. 19438, 20159, 25232, 29101.

Yucca rigida (Engelm.) Trel. 23370, 28519, 29384.

Yucca treculeana Carrière. 28358, 29383.

\section{Family Asteraceae}

Acmella radicans (Jacq.) R.K. Jansen. 3305, 5728, 11013, 12240.

Acmella repens (Walter) Rich. 1913, 2530, 5115, 5645, 14847, 20533.

Acourtia cordata (Cerv.) B.L. Turner. 29860.

Acourtia coulteri (A. Gray) Reveal \& R.M. King. 22983, 24718 (Type of Vernonia zaragozana B.L. Turner).

Acourtia cuernavacana (B.L. Rob. \& Greenm.) Reveal \& R.M. King. 17495bis.

Acourtia dugesii (A. Gray) Reveal \& R.M. King. 2934, 3069, 3086.

Acourtia hidalgoana B.L. Turner. 22530, 23491, 27286. Acourtia michoacana (B.L. Rob.) Reveal \& R.M. King. 12760.

Acourtia nana (A. Gray) Reveal \& R.M. King. 19853, 21940,
25997, 28735.

Acourtia parryi (A. Gray) Reveal \& R.M. King. 19751, 23022, 25998, 29119.

Acourtia platyptera (B.L. Rob.) Reveal \& R.M. King. 2952, 11306.

Acourtia pringlei (B.L. Rob. \& Greenm.) Reveal \& R.M. King. 2782, 2944, 8722, 8783, 14912.

Acourtia purpusii (Brandegee) Reveal \& R.M. King. 19058, 20717, 20869, 21514, 21714, 23607, 23952.

Acourtia queretarana B.L. Turner. 22600.

Acourtia reticulata (Lag. ex D. Don) Reveal \& R.M. King. 2398, 2618.

Acourtia runcinata (Lag. ex D. Don) B.L. Turner. 20375, 20818, 21886, 22001, 22802, 23853, 29469.

Acourtia scaposa (S.F. Blake) B.L. Turner. 15838 (TYPUS).

Acourtia simulata (S.F. Blake) Reveal \& R.M. King. 13654 (TYPUS).

Acourtia tomentosa (Brandegee) Reveal \& R.M. King. 23934

(Type of Acourtia hintoniorum B.L. Turner), 27229, 27285, 27288.

Acourtia turbinata (Lex.) DC. 260, 3277, 8968, 17516bis. Acourtia wrightii (A. Gray) Reveal \& R.M. King. 16599, 18459, 18551, 18585, 18661, 18995, 19778, 19860, 19915, 22009, 23027, 25834, 29470.

Achillea millefolium L. 509, 3997, 17110, 17869, 17899, 18590.

Adenophyllum aurantium (L.) Strother. 11597, 12953.

Adenophyllum cancellatum (Cass.) Villarreal. 20883.

Adenophyllum porophyllum (Cav.) Hemsl. 1747, 12522.

Ageratina brevipes (DC.) R.M. King \& H. Rob. 29770.

Ageratina calaminthifolia (Kunth) R.M. King \& H. Rob. 17882, 19059, 19814, 19859, 19895, 19928, 19989, 20070, 21653, 22364, 23595, 23751, 23887, 24951, 25160, 27960, 28006, 28038, 28059, 28074, 28129, 28143, 28225, 28227. Ageratina deltoidea (Jacq.) R.M. King \& H. Rob. 29795. Ageratina petiolaris (Moc. \& Sessé ex DC.) R.M. King \& H. Rob. 29826.

Ageratina amblyolepis (B.L. Rob.) R.M. King \& H. Rob. 11123 (Type of Ageratina hintonii R.M. King \& H. Rob.), 11125.

Ageratina areolaris (DC.) Gage ex B.L. Turner. 1955, 2320, 2562, 8685, 9889, 11079, 11140, 14811bis, 14879, 15614, 26297, 26341, 26348, 26440.

Ageratina atrocordata (B.L. Rob.) R.M. King \& H. Rob. 26360.

Ageratina bellidifolia (Benth.) R.M. King \& H. Rob. 8688, 11171, 13555, 14945.

Ageratina calophylla (Greene) R.M. King \& H. Rob. ex Molinari \& Mayta. 17311, 21595, 21744, 24001.

Ageratina campyloclada (B.L. Rob.) R.M. King \& H. Rob. 17029, 17272, 18160, 18815, 20214, 20268, 27535.

Ageratina cardiophylla (B.L. Rob.) R.M. King \& H. Rob. 9237.

Ageratina conspicua R.M. King \& H. Rob. 364, 21641, 21689, 21739, 21742, 23962, 25036, 25077.

Ageratina chiapensis (B.L. Rob.) R.M. King \& H. Rob. 17536bis. 
Ageratina choricephala (B.L. Rob.) R.M. King \& H. Rob. 129.

Ageratina deltoidea (Jacq.) R.M. King \& H. Rob. 2740, 8412 .

Ageratina espinosarum (A. Gray) R.M. King \& H. Rob. 17700, 19925, 19986, 20789, 21511, 21569, 21718, 21763, 23783, 27769, 28131, 28149.

Ageratina gypsophila B.L. Turner. 18779 (TYPUS), 18782 , 19267, 20496, 27014, 27789, 27992, 28035, 29059.

Ageratina havanensis (Kunth) R.M. King \& H. Rob. 16842, 16909, 17443, 17701, 17705, 19096, 19929, 19990, 20000, 20840, 21706, 21719, 21722, 21730, 21748, 21757, 21765, 22004, 22182, 23805, 23886, 24948, 24956, 24992, 25020, 28120, 28130, 28155, 28174.

Ageratina herbacea (A. Gray) R.M. King \& H. Rob. 25808.

Ageratina hernandezii B.L. Turner. 23828.

Ageratina ilicifolia B.L. Turner. 20837 (TYPUS), 27790, 28331.

Ageratina ligustrina (DC.) R.M. King \& H. Rob. 345, 3321, 14236, 17439, 22494, 22502, 23808, 23922, 24822, 24914, 25063, 25064, 25070, 25104, 25110, 25111.

Ageratina macdonaldii B.L. Turner. 26139, 26515.

Ageratina mairetiana (DC.) R.M. King \& H. Rob. 3336, 8908, 17535bis, 26818.

Ageratina miahuatlana B.L. Turner. 26062, 26258, 26304 (TYPUS), 26685, 26701.

Ageratina muelleri (Sch. Bip. ex Klatt) R.M. King \& H. Rob. 2374, 2710, 2875, 5220, 14764, 14767, 17452bis.

Ageratina neohintoniorum B.L. Turner. 8848 (TYPUS).

Ageratina nesomii B.L. Turner. 21761, 23663, 25628, 25809, 25812, 27701.

Ageratina oligocephala (DC.) R.M. King \& H. Rob. 17187.

Ageratina oreithales (Greenm.) B.L. Turner. 18932, 19075, 22435, 27235.

Ageratina parayana (J. Espinosa) B.L. Turner. 26320.

Ageratina pazcuarensis (Kunth) R.M. King \& H. Rob. 15570, 21606, 26022, 26051, 26064, 26119, 26226, 26406, 26847. Ageratina petiolaris (Moc. \& Sessé ex DC.) R.M. King \& H. Rob. 19318, 19354, 20127, 21792, 21818, 24013, 25210.

Ageratina pichinchensis (Kunth) R.M. King \& H. Rob. 13578, 22482, 22515, 22655, 23790, 23954, 24116, 24929, 25176, 25193, 28118.

Ageratina potosina B.L. Turner. 18910, 22408.

Ageratina pringlei (B.L. Rob. \& Greenm.) R.M. King \& H. Rob. 26240.

Ageratina rhomboidea (Kunth) R.M. King \& H. Rob. 21610, 22131, 22446, 22511, 22517, 23811, 23980.

Ageratina riparia (Regel) R.M. King \& H. Rob. 26386.

Ageratina rubricaulis (Kunth) R.M. King \& H. Rob. 2440, 14801, 17439bis.

Ageratina saltillensis (B.L. Rob.) R.M. King \& H. Rob. 18346, 18612, 18709, 19001, 19269, 19825, 19903, 20739, 21257, 21627, 21652, 21688, 21713, 21740, 24936, 27973, 27987, 28001, 28070, 28099.

Ageratina scorodonioides (A. Gray) R.M. King \& H. Rob. 17185, 17733, 18351, 18928, 19258, 19818, 19826, 19906,
20493, 21590, 28054, 28055, 28209.

Ageratina vernalis (Vatke \& Kurtz) R.M. King \& H. Rob. 17465, 23960, 25076, 25090, 25091, 25101, 26097, 26124, 26266, 26402, 26410, 26411.

Ageratina vernicosa (Sch. Bip. ex Greenm.) R.M. King \& H. Rob. 13498 (Type of Ageratina hintoniorum B.L. Turner). Ageratina viburnoides (DC.) R.M. King \& H. Rob. 17729, 20043, 21668, 23829, 23913, 25035, 25106.

Ageratina viejoana B.L. Turner. 22484 (TYPUS), 22504, 23807, 23959, 27279.

Ageratina wrightii (A. Gray) R.M. King \& H. Rob. 16885, 18597, 19864, 20708, 21738, 24953, 25685, 25710.

Ageratina zapalinama B.L. Turner. 20499 (TYPUS).

Ageratina zaragozana B.L. Turner. 23977 (TYPUS).

Ageratum corymbosum Zuccagni. 18053, 29706.

Ageratum corymbosum Zuccagni. 1502, 1815, 1863, 2066, 8382, 12456, 17474bis, 17629, 19000, 19252, 19727, 19850, 19988, 20481, 20705, 20794, 22327, 22473, 23710, 23767, 25631, 28148.

Ageratum houstonianum Mill. 5537.

Ageratum microcephalum Hemsl. 26321.

Aldama buddlejiformis (DC.) E.E. Schill. \& Panero. 9870, 9871, 14870, 15575, 29864.

Aldama cordifolia (A. Gray) E.E. Schill. \& Panero. 16883, 19210, 25429, 25465, 25542, 25624, 27287, 27937, 28098. Aldama dentata La Llave. 512, 2041, 2517, 13622.

Aldama excelsa (Willd.) E.E. Schill. \& Panero. 29757, 29790bis.

Aldama ghiesbreghtii (Hemsl.) E.E. Schill. \& Panero. 5268, 8384.

Aldama morelensis (Greenm.) E.E. Schill. \& Panero. 2463. Aldama palmeri (A. Gray) E.E. Schill. \& Panero. 12441 (Type of Viguiera palmeri var. coalcomana B.L. Turner). Alomia ageratoides Kunth. 3347 (Type of Alomia hintonii R.M. King \& H. Rob.).

Alloispermum integrifolium (DC.) H. Rob. 11313, 26296, 26450, 26829.

Alloispermum longiradiatum (Urbatsch \& B.L. Turner) B.L. Turner. 10449, 10472, 14359 (TYPUS).

Alloispermum palmeri (S. Watson ex A. Gray) C.F. Fernández \& Urbatsch. 15041.

Alloispermum scabrum (Lag.) H. Rob. 1503, 1827, 7958, $8133,8190,12338,12403,12762,13187,13472,14802$, 15127, 15664, 17431bis, 21642, 22490, 23452, 23791.

Amauriopsis autumnalis (W.L. Ellison) B.L. Turner. 17688, 20779, 23678, 24746, 24987, 25900, 28179, 28563.

Ambrosia confertiflora DC. 16684, 19595, 20635, 24973, 27570, 28659.

Aphanactis macdonaldii B.L. Turner. 26138, 26148, 26582, 26792.

Aphanostephus ramosissimus DC. 29840.

Aphanostephus ramosissimus DC. 5827, 6003, 17145, 17827, 18197, 19497, 19578, 19637, 24157, 28345, 28761, 29098. Aquilula riskindii (B.L. Turner \& G. Langford) G.L. Nesom. 18192, 19683, 20261, 22718.

Archibaccharis asperifolia (Benth.) S.F. Blake. 3266, 8836, 14784.

Archibaccharis serratifolia (Kunth) S.F. Blake. 29801. 
Archibaccharis asperifolia (Benth.) S.F. Blake. 2461, 2907, 11138.

Archibaccharis auriculata (Hemsl.) G.L. Nesom. 8823, 15405.

Archibaccharis hieracioides (S.F. Blake) S.F. Blake. 3271, 11124.

Archibaccharis hirtella (DC.) Heering. 3089, 17422bis, 17449bis, 17503bis.

Archibaccharis macdonaldii G.L. Nesom. 26143.

Archibaccharis schiedeana (Benth.) J.D. Jacks. 2110, 2832. Archibaccharis serratifolia (Kunth) S.F. Blake. 2730, 2898, 17499bis, 22254, 23444, 26019, 26094, 26268, 26365, 27270.

Artemisia carruthii Alph. Wood. ex Carruth. 18575.

Artemisia klotzschiana Besser. 16882, 19838, 20763.

Artemisia ludoviciana Nutt. 1847, 18347, 18576, 19072, 19817, 19880, 19918, 20080, 20815, 21355, 24946, 25533, 29026.

Asanthus thyrsiflorus (A. Gray) R.M. King \& H. Rob. 2973.

Astranthium beamanii De Jong. 17006, 18547, 19463, 25493, 27182, 27257.

Astranthium ciliatum (Raf.) G.L. Nesom. 24195, 24416.

Astranthium splendens De Jong. 17355, 19843, 21248, 21272, 21291, 21619, 22192, 22220, 22953, 23938, 27236.

Astranthium xylopodum Larsen. 609, 13959.

Axiniphyllum pinnatisectum (Paul G. Wilson) B.L. Turner. 9756, 11289 (TYPUS).

Axiniphyllum sagittalobum B.L. Turner. 14761 (TYPUS).

Aztecaster matudae (Rzed.) G.L. Nesom. 20225, 20231, 29102.

Baccharis pteronioides DC. 29830.

Baccharis conferta Kunth. 10172.

Baccharis crassicuneata G.L. Nesom. 17017, 19802, 19822, 19823, 21517, 21567, 21716, 24647, 27941, 27962, 28100.

Baccharis heterophylla Kunth. 348, 435, 2833, 6519, 10166, 18035, 18066, 18771, 26267, 26520, 26521, 26571, 27683, 27684, 28228.

Baccharis multiflora Kunth. 2711, 2731, 2850, 13361, 26067, 26128, 26202, 26222, 26394, 26403, 26407, 26604, 26619.

Baccharis neglecta Britton. 17638, 21538, 23398, 23834, 28105, 28106.

Baccharis pteronioides DC. 16607, 16746, 18419, 18656, 19419, 20248, 27554, 29620, 29621.

Baccharis salicifolia (Ruiz \& Pav.) Pers. 1393, 2131, 2147, 13579, 16671, 17968, 20875.

Baccharis salicina Torr. \& A. Gray. 20514, 20515.

Baccharis sordescens DC. 20895.

Baccharis texana (Torr. \& A. Gray) A. Gray. 18962, 19040.

Baccharis thesioides Kunth. 18933, 20440, 20489, 22272 , 27085, 27251.

Baccharis trinervis (Lam.) Pers. 3170, 12981, 14021.

Bahia absinthifolia Benth. 16578, 18446, 18605, 19627, 19708, 19744, 19785, 19943, 20617, 21506, 21891, 23014, 23344, 23732, 24730, 24748, 25515, 28384, 28758, 28862, 28879, 29002.

Bahia schaffneri S. Watson. 21108, 22328.
Barkleyanthus salicifolius (Kunth) H. Rob. \& Brettell. 297, 3113, 7614, 16732, 17027, 18377, 19323, 29834.

Bartlettina hintonii R.M. King \& H. Rob. 15396 (TYPUS). Bartlettina oresbia (B.L. Rob.) R.M. King \& H. Rob. 3454, 3636, 8954, 8995.

Bartlettina sordida (Less.) R.M. King \& H. Rob. 17537bis. Berlandiera lyrata Benth. 18394, 18672, 19198, 19891, 21172, 21338, 21368, 23053, 28010, 28205, 29016.

Berlandiera $x$ macrophylla (A. Gray) M.E. Jones. 18261, 18468.

Bidens odorata Cav. 29787, 29823.

Bidens pilosa L. 12521.

Bidens acrifolia Sherff. 9388.

Bidens alba (L.) DC. 26527.

Bidens angustissima Kunth. 19018.

Bidens anthemoides (DC.) Sherff. 4003, 4327.

Bidens aurea (Aiton) Sherff. 1573, 8310, 8436, 12232, 15481, 19884, 25531.

Bidens bicolor Greenm. 26021.

Bidens bigelovii A. Gray. 18587, 18589.

Bidens ferulifolia (Jacq.) DC. 17634.

Bidens gracillima Sherff. 4546 (TYPUS).

Bidens hintonii (Sherff) Melchert. 9379 (TYPUS), 11510.

Bidens longistyla C.R. Hart. 19179, 19666, 19764, 20711, 21571, 21572, 21708, 23667, 25696.

Bidens mexicana Sherff. 11642, 12584.

Bidens minensis Sherff. 9453 (TYPUS).

Bidens odorata Cav. 16853, 17446, 17633, 19641, 19717 , 19718, 19788, 19870, 21591, 22514, 23837, 25028, 25537.

Bidens ostruthioides (DC.) Sch. Bip. 2737, 2897, 13521, 26010, 26029, 26342.

Bidens pilosa L. 1508, 2109, 2666, 7545, 12846, 12971, 14895, 15304, 17479bis, 19787, 21400, 21522, 22500.

Bidens pringlei Greenm. 15588.

Bidens riparia Kunth. 8551, 11475.

Bidens rostrata Melchert. 1933, 2126, 5081.

Bidens saltillensis Melchert. 20483.

Bidens serrulata (Poir.) Desf. 1889, 4929, 15256, 19791, 20086, 21614.

Bidens squarrosa Kunth. 2229, 25015, 25137.

Bidens triplinervia Kunth. 11116, 12371, 14455, 15569, 18911, 19074, 19805, 19844, 21361, 21696, 21704, 22385, 22402, 22441, 22493, 22545, 22588, 23453, 25088, 25555, 26083, 26084, 26129, 26155, 26232, 26235, 26269, 26591, 26657, 26762, 26764, 28051.

Blumea viscosa (Mill.) V.M. Badillo. 3391, 5451, 13624.

Boeberoides grandiflora (DC.) Strother. 8604, 8758, 9827. Brickellia oliganthes (Less.) A. Gray. 15763.

Brickellia scoparia (DC.) A. Gray. 29822.

Brickellia secundiflora (Lag.) A. Gray. 29809.

Brickellia aramberrana B.L. Turner. 20031, 20050 (TYPUS).

Brickellia coahuilensis (A. Gray) Harc. \& Beaman. 19030.

Brickellia coulteri A. Gray. 16871, 23573, 24763, 27427, 29597.

Brickellia eupatorioides (L.) Shinners. 3067, 16765, 18571, 19812, 19837, 19867, 23676, 23684, 23929, 24853, 24939, 24996, 25060, 25532, 27258, 27522, 27649, 28003, 28170, 
28788, 28789, 29032, 29033.

Brickellia filipes B.L. Rob. 2977, 5723.

Brickellia glomerata Fernald. 2895.

Brickellia glutinosa A. Gray. 29332, 29373.

Brickellia grandiflora (Hook.) Nutt. 19039, 19268, 21280, 21683, 21743, 22550, 22885, 23907, 24909, 25085.

Brickellia hintoniorum B.L. Turner. 17924, 17970 (TYPUS), 18904, 20430, 22250, 22418.

Brickellia laciniata A. Gray. 17713, 18042, 19105, 19846, 19926, 19950, 19970, 21576, 23548, 23892, 24942.

Brickellia lemmonii A. Gray. 16911, 18319, 18610, 18628, 18760, 19057, 19924, 20348, 20543, 20707, 20849, 21705, 21727, 21728, 23292, 23594, 23604, 23661, 23687, 23734, 23885, 24508, 25920, 27888, 28024, 28187, 28229, 28916, 29040.

Brickellia nesomii B.L. Turner. 17257, 18577, 19841, 24932, 25548.

Brickellia oliganthes (Less.) A. Gray. 2468, 2871, 20366.

Brickellia orizabaensis Klatt. 3269, 11233.

Brickellia palmeri A. Gray. 18630, 18647.

Brickellia paniculata (Mill.) B.L. Rob. 13601, 20033, 20039.

Brickellia pavonii (A. Gray) B.L. Turner. 2510, 6873.

Brickellia pedunculosa (DC.) Harc. \& Beaman. 15707.

Brickellia pendula (Schrad.) A. Gray. 3063, 3286, 17517bis.

Brickellia scoparia (DC.) A. Gray. 2869, 14872.

Brickellia secundiflora (Lag.) A. Gray. 15695, 19820, 23820 , 24866, 25579, 27488.

Brickellia spinulosa (A. Gray) A. Gray. 22722.

Brickellia squarrosa B.L. Rob. \& Seaton. 3351.

Brickellia subuligera (S. Schauer) B.L. Turner. 19979, 21682, 25045, 25638, 27770.

Brickellia urolepis S.F. Blake. 25936.

Brickellia veronicifolia (Kunth) A. Gray. 16886, 17471, 18075, 19827, 19848, 19854, 19876, 19897, 19968, 20049, 20073, 21736, 22572, 23675, 23891, 24867, 24938, 24945, 25708, 25803, 28204, 28970.

Calanticaria brevifolia (Greenm.) E.E. Schill. \& Panero. 16583, 23536.

Calanticaria greggii (A. Gray) E.E. Schill. \& Panero. 18094, 18567, 18568, 18774, 19730, 20715, 23583, 27461, 27901, 28933.

Calea ternifolia Kunth. 1601, 2335, 10831, 20586, 21680, 24885.

Calea urticifolia (Mill.) DC. 188, 2948, 3038, 9843, 12932 , 13595, 14811.

Calyptocarpus vialis Less. 667, 2299, 17525, 17525bis, 17693, 19794, 19946, 20161, 20522, 24082, 24354, 28988.

Carminatia tenuiflora DC. 2228, 4871, 6870, 14949, 15228, 22524.

Centaurea iberica Trevir. ex Spreng. 20465.

Cirsium acrolepis (Petr.) G.B. Ownbey. 20403, 20943, 21927, 22942, 23135, 24229, 25259, 25380, 25387.

Cirsium conspicuum (G. Don) Sch. Bip. 12796, 13874.

Cirsium faucium Petr. 3250 (TYPUS), 8921.

Cirsium grahamii A. Gray. 27609.

Cirsium horridulum Michx. 21942.
Cirsium mexicanum DC. 24105, 28655.

Cirsium pringlei (S. Watson) Petr. 16785, 17117, 17251, 17560, 18909, 19171, 20448, 21011, 21031, 21336, 22282, 22823, 24339, 25246, 25401, 25545, 28287.

Cirsium radians Benth. 3500 .

Cirsium texanum Buckley. 16881, 19479, 19486, 20017, 20365, 20585, 20614, 20918, 24236, 27807.

Cirsium undulatum (Nutt.) Spreng. 17927, 18561, 20246, 20470, 20968.

Clibadium arboreum Donn. Sm. 14698.

Conoclinium betonicifolium (Mill.) R.M. King \& H. Rob. 17851, 18553, 19959, 20338, 20538, 21935, 21953, 22063, 22064, 22372.

Conoclinium dissectum A. Gray. 16682, 16867, 19752, 20634, 28679.

Conoclinium mayfieldii T.F. Patterson. 25030.

Conyza coronopifolia Kunth. 29844.

Conyza bonariensis (L.) Cronquist. 3137.

Conyza canadensis (L.) Cronquist. 5672, 12878, 12975, 18761, 18775, 19515, 19568, 20517, 21395.

Conyza coronopifolia Kunth. 14373, 15015, 22123, 23211, 23459, 26528, 26601, 26675, 26710.

Conyza microcephala Hemsl. 26749.

Coreopsis petrophila A. Gray. 9855, 11254.

Coreopsis petrophiloides B.L. Rob. \& Greenm. 499, 2841, 8796.

Cosmos parviflorus (Jacq.) Pers. 29732.

Cosmos scabiosoides Kunth. 18005.

Cosmos bipinnatus Cav. 525, 4943, 4969, 4990, 12420, 15605.

Cosmos carvifolius Benth. 15534.

Cosmos caudatus Kunth. 1786, 4790, 6745, 12632.

Cosmos crithmifolius Kunth. 2076, 8686, 17413bis, 22111 , 23289.

Cosmos diversifolius Otto. 14804, 22109, 22224, 22557, 23465, 26654, 26739.

Cosmos mattfeldii Sherff. 9369.

Cosmos modestus Sherff. 8287.

Cosmos parviflorus (Jacq.) Pers. 4893, 8435.

Cosmos purpureus (DC.) Hemsl. 1700 (Type of Cosmos purpureus var. flavidiscus Sherff), 1896, 4992, 10666.

Cosmos scabiosoides Kunth. 1554, 6581, 12408, 15441.

Cosmos schaffneri Sherff. 4865.

Cosmos sulphureus Cav. 1986, 2061, 6639, 9606, 13592, 15059, 15305, 15636, 20023, 20065.

Cotula mexicana (DC.) Cabrera. 8002.

Critonia hebebotrya DC. 2486, 2551.

Critonia lozanoana (B.L. Rob.) R.M. King \& H. Rob. 17639.

Critonia morifolia (Mill.) R.M. King \& H. Rob. 24514 , 24518, 25214.

Critonia quadrangularis (DC.) R.M. King \& H. Rob. 3142, 12822.

Cymophora accedens (S.F. Blake) B.L. Turner \& A.M. Powell. 12850, 12884 (TYPUS).

Cymophora hintonii B.L. Turner \& A.M. Powell. 12590 (TYPUS).

Cymophora pringlei B.L. Rob. \& Greenm. 12226. 
Chaetopappa bellioides (A. Gray) Shinners. 18990, 21057, 22022, 23330, 23528, 24460, 28949, 29052, 29116.

Chaetopappa ericoides (Torr.) G.L. Nesom. 16716, 18080, 18198, 18421, 19130, 19699, 19723, 19834, 19857, 19900, 21080, 23862, 25481, 25765, 25857, 27018, 27089, 28033, 28585, 28681, 28690, 29153, 29193.

Chaetopappa parryi A. Gray. 16707, 18482, 18956, 19017, 19146, 20219, 20265, 25852.

Chaptalia hintonii Bullock. 3098 (TYPUS), 3465, 8228, 8562, 8662.

Chaptalia hololeuca Greene. 18250, 18795, 18958, 20114, 20121, 20140, 20228, 21039, 21788, 22658, 22750, 22819, 22827, 23091, 23842, 25642, 28018, 28904, 29107.

Chaptalia lyratifolia Burkart. 16706, 18955, 20195, 20227, 21074, 22737, 23953, 25927.

Chaptalia nutans (L.) Pol. 1006, 1179, 13919, 20032, 20166, 20805, 21203, 21369, 21659, 22594, 23620, 23873, 23908, 24845, 25540, 25570, 28159, 28574, 28951, 29120, 29198. Chaptalia transiliens G.L. Nesom. 19685, 20218, 21328, 21631.

Chionolaena salicifolia (Bertol.) G.L. Nesom. 3260, 8825, 11120.

Chloracantha spinosa (Benth.) G.L. Nesom. 3178, 5610.

Chromolaena pulchella (Kunth) R.M. King \& H. Rob. 18110, 29758.

Chromolaena bigelovii (A. Gray) R.M. King \& H. Rob. 20367.

Chromolaena collina (DC.) R.M. King \& H. Rob. 2409, 2475, 2582, 3007, 14839, 19999.

Chromolaena haenkeana (DC.) R.M. King \& H. Rob. 17507 bis.

Chromolaena odorata (L.) R.M. King \& H. Rob. 2465, 9821, 12626, 14841, 17703, 19934, 19957, 20038, 20839, 20872, 22467, 23875, 23987, 24453, 24994, 24998.

Chromolaena ovaliflora (Hook. \& Arn.) R.M. King \& H. Rob. 10837, 12944.

Chromolaena pulchella (Kunth) R.M. King \& H. Rob. 12180.

Chromolaena tamaulipasensis (R.M. King \& H. Rob.) B.L. Turner. 23884.

Chrysactinia acerosa S.F. Blake. 22003, $22178,27004$.

Chrysactinia mexicana A. Gray. 16567, 16629, 17756, 18677, 19610, 22025, 28023.

Chrysactinia pinnata S. Watson. 19232, 19973.

Chrysactinia truncata S. Watson. 16705, 17569, 23061, 24369.

Chrysanthellum americanum (L.) Vatke. 1365, 1610, 4434, 6365.

Chrysanthellum indicum DC. 8185, 12239.

Chrysanthellum involutum Paul G. Wilson. 6269, 6417 (TYPUS), 6977, 8032.

Chrysanthellum michoacanum B.L. Turner. 12058.

Dahlia coccinea Cav. 18004.

Dahlia merckii Lehm. 17993.

Dahlia atropurpurea P.D. Sorensen. 10665.

Dahlia coccinea Cav. 854, 2028, 7965, 7999, 9221, 11350, 12266, 14496, 15257, 15499, 15936, 15954, 17407bis, 19190, 19761, 20415, 23095, 23227, 25436, 25558.
Dahlia foeniculifolia Sherff. 23414, 24581.

Dahlia hintonii Sherff. 14499 (TYPUS).

Dahlia pinnata Cav. 11033, 11083.

Dahlia rudis P.D. Sorensen. 1885, 8332.

Dahlia scapigera (A. Dietr.) Knowles \& Westc. 1039, 13239.

Dahlia sorensenii H.V. Hansen \& Hjert. 26020, 26088, 26246, 26695.

Dahlia tenuicaulis P.D. Sorensen. 12364, 26052, 26633.

Dahlia tubulata P.D. Sorensen. 17188, 17414, 17567, 18344,

19025, 19178, 21310, 22436, 23152, 25566, 25605, 27260, 28567.

Decachaeta haenkeana DC. 9658, 12440, 15236.

Decachaeta ovatifolia (DC.) R.M. King \& H. Rob. 2561, 2580, 9829, 12652.

Decachaeta pyramidalis (B.L. Rob.) S.D. Sundb., C.P. Cowan \& B.L. Turner. 5054, 15225.

Delilia biflora (L.) Kuntze. 1980, 4718, 15312.

Dendroviguiera adenophylla (S.F. Blake) E.E. Schill. \& Panero. 19043, 19050, 21342, 21375, 21707, 23431, 25102, 27269, 27282.

Dendroviguiera neocronquistii (B.L. Turner) E.E. Schill. \& Panero. 26378, 26727.

Dendroviguiera puruana (Paray) E.E. Schill. \& Panero. 11653.

Dendroviguiera sphaerocephala (DC.) E.E. Schill. \& Panero. $2201,9624$.

Desmanthodium fruticosum Greenm. 1675, 1860, 2015, 2212, 9559, 13932, 14628, 15123.

Desmanthodium hintoniorum B.L. Turner. 26409 (TYPUS). Desmanthodium ovatum Benth. 2758, 4635.

Diaperia verna (Raf.) Morefield. 29479.

Dicranocarpus parviflorus A. Gray. 18638, 19538, 19562, 19638, 19655, 20642, 23640, 27616, 28523, $29370,29579$.

Digitacalia hintoniorum B.L. Turner. 12572 (TYPUS).

Dyssodia papposa (Vent.) Hitchc. 18055.

Dyssodia pinnata (Cav.) B.L. Rob. 19229.

Dyssodia papposa (Vent.) Hitchc. 2839, 16852, 17121, 17447, 19539, 19719, 19720, 19790, 25574, 25575.

Dyssodia pinnata (Cav.) B.L. Rob. 17445, 17463, 17477, 18437, 18552, 18734, 18997, 19202, 19600, 19668, 19684, 20044, 20087, 20187, 20488, 23598, 24353, 24803, 24943, 25619, 27690, 28013, 28976.

Dyssodia tagetiflora Lag. 6227.

Eclipta prostrata (L.) L. 4171, 5516, 10546, 11970, 13966, 14576, 29230.

Electranthera mutica (DC.) Mesfin, D.J. Crawford \& Pruski. 26122, 26327, 26357, 26603, 26631, 26724 (Type of Coreopsis mutica var. miahuatlana B.L. Turner), 26725, 26839. Elephantopus mollis Kunth. 2635, 7182, 14992.

Engelmannia peristenia (Raf.) Goodman \& C.A. Lawson. 18472, 20016, 20394, 21189, 27524.

Erechtites hieraciifolius (L.) Raf. ex DC. 2271, 3654, 7603. Eremosis corymbosa (Mill.) Pruski. 12894, 13651, 15762.

Eremosis obtusa Gleason. 17424, 17704, 19010, 19036, 20045, 23411, 23897, 25048, 25121.

Eremosis pallens (Sch. Bip.) Gleason. 2377, 3132 (TYPUS). 
Eremosis tomentosa (Lex.) Gleason. 3108, 3231, 13542. Erigeron delphinifolius Willd. 18029.

Erigeron gypsoverus G.L. Nesom. 28917.

Erigeron longipes DC. 29845.

Erigeron pattersonii G.L. Nesom. 29899.

Erigeron basilobatus S.F. Blake. 16943, 17415, 17871, 17986, 18540, 18920, 20997, 21260, 21607, 22098, 23151, 25606.

Erigeron bigelovii A. Gray. 19620.

Erigeron divergens Torr. \& A. Gray. 20475.

Erigeron dryophyllus A. Gray. 17732, 17777, 19122, 19345, 19801, 20042, 22278, 22595, 24046, 25252.

Erigeron flagellaris A. Gray. 19911, 24391, 27193.

Erigeron gypsoverus G.L. Nesom. 18474, 20949, 21485, 22041, 28588.

Erigeron heleniae G.L. Nesom. 28412 (TYPUS), 28611, 28636.

Erigeron hintoniorum G.L. Nesom. 18868 (TYPUS), 18907.

Erigeron karvinskianus DC. 226, 916, 7687, 11820.

Erigeron longipes DC. 561, 624, 3322, 6053, 12391, 13604, 13923, 14385.

Erigeron metrius S.F. Blake. 18926, 24118.

Erigeron modestus A. Gray. 18690, 20040, 20594, 27381.

Erigeron onofrensis G.L. Nesom. 17390.

Erigeron palmeri A. Gray. 22150, 22210, 23088, 23301, 23463.

Erigeron pattersonii G.L. Nesom. 25641, 25874, 28718, 28724.

Erigeron polycephalus (Larsen) G.L. Nesom. 11314.

Erigeron potosinus Standl. 17199, 18579, 18908, 20455.

Erigeron pubescens Kunth. 17908, 18409, 18516, 18519, 18984, 19245, 19693, 19695, 19725, 19779, 20093, 20128, 20282, 20315, 20349, 20399, 20454, 20604, 20732, 20814, 21072, 21196, 21585, 22899, 23120, 23288, 24470, 24566, 24629, 24949, 25460, 25860, 28016, 28680, 28715, 29000, 29031.

Erigeron quiexobrensis G.L. Nesom. 26212, 26793.

Erigeron veracruzensis G.L. Nesom. 20025, 20368, 20555, 21373, 22890, 23074, 23149, 24563, 24607, 29023, 29105.

Eryngiophyllum pinnatisectum P.G. Wilson. 6598 (TYPUS), 8020, 9164.

Eupatoriastrum triangulare (DC.) B.L. Rob. 8542, 8642, 11029, 11441, 14634.

Flaveria angustifolia (Cav.) Pers. 5844.

Flaveria anomala B.L. Rob. 18068, 18642, 19508, 20069, 20638, 22360, 27617, 27620, 28672, 28759, 28999.

Flaveria chlorifolia A. Gray. 17865, 21951.

Flaveria oppositifolia (DC.) Rydb. 16879, 18036, 18766, 20471, 20599, 23338, 23637, 25993, 27618, 28044, 29489.

Flaveria palmeri J.R. Johnst. 19442, 19564, 29320.

Flaveria trinervia (Spreng.) C. Mohr. 5478, 7284, 16863, 19547, 29232, 29281.

Fleischmannia arguta (Kunth) B.L. Rob. 11328.

Fleischmannia arguta (Kunth) B.L. Rob. 10288, 11749, 14148.

Fleischmannia holwayana (B.L. Rob.) R.M. King \& H. Rob. 2815, 5243.
Fleischmannia porphyranthema (A. Gray) R.M. King \& H. Rob. 16889, 16890, 19875, 19987, 20036, 20047, 20702, 20750, 21598, 21600, 21663, 22475, 22535, 22631, 23781, 23782, 23789, 23814, 23901, 24019, 24794, 25024, 25061, 25105, 25116, 28167, 28734.

Fleischmannia pycnocephala (Less.) R.M. King \& H. Rob. 11268, 13617, 17451, 19203, 19238, 19249, 19933, 20056. Fleischmanniopsis leucocephala (Benth.) R.M. King \& H. Rob. 14042.

Florestina pedata (Cav.) Cass. 17996.

Florestina lobata B.L. Turner. 1261 (TYPUS), 4321, 9122. Florestina pedata (Cav.) Cass. 12052, 12119, 19056.

Florestina tripteris DC. 6426, 16690, 20619, 24269, 24759, 27243, 28927.

Flourensia cernua DC. 18072, 19495, 19721, 28302.

Flourensia ilicifolia Brandegee. 22616.

Flourensia laurifolia DC. 25003.

Flourensia monticola M.O. Dillon. 20257, 20738, 21095, 24950.

Flyriella parryi (A. Gray) R.M. King \& H. Rob. 25788, 27084.

Flyriella stanfordii R.M. King \& H. Rob. 17544, 22099, 22190, 22191, 22519, 22940, 23212, 23239, 24612, 24707.

Gaillardia candelaria B.L. Turner. 29410.

Gaillardia candelaria B.L. Turner. 29346 (Type of Gaillardia candelaria var. mikemoorei B.L. Turner), 29361.

Gaillardia comosa A. Gray. 19626, 21941, 23866, 28737.

Gaillardia mexicana A. Gray. 16791, 18264, 18484, 19168, 19832, 19985, 20026, 20292, 28341, 28937.

Gaillardia pinnatifida Torr. 16630.

Gaillardia turneri Averett \& A.M. Powell. 29275.

Galeana pratensis (Kunth) Rydb. 1419, 2142, 4423, 9556, 15160.

Galinsoga parviflora Cav. 9548.

Galinsoga longipes Canne. 11534.

Galinsoga parviflora Cav. 2056, 2295, 2431, 12429, 12996, 17441bis, 19789, 19883, 19914, 21363, 27221, 28989.

Gamochaeta americana (Mill.) Wedd. 14454, 26661.

Gamochaeta coarctata (Willd.) Kreguélen. 3494, 6231.

Gamochaeta falcata (Lam.) Cabrera. 18546.

Gamochaeta purpurea (L.) Cabrera. 22105, 22293, 23460.

Grindelia greenmanii Steyerm. 17890, 19831, 19865, 20205, 20880, 20996, 21010, 24382, 24957, 25337, 25388, 25428, 27274, 27785, 29061.

Grindelia hintoniorum G.L. Nesom. 18666 (TYPUS).

Grindelia inuloides Willd. 11941, 17148, 17258, 17296, 18531, 20398, 20735, 20995, 21193, 21428, 27661.

Grindelia obovatifolia S.F. Blake. 18618, 20024, 20518, 21161, 21231, 21252, 21316, 21434, 21446, 22263, 22383, 22398, 23402.

Grindelia squarrosa (Pursh) Dunal. 19518, 19581, 20291, 27658.

Grindelia subdecurrens DC. 28344.

Grindelia tenella Steyerm. 24524, 24783, 28206.

Grindelia turneri G.L. Nesom. 17760, 19481, 22268, 25402, 27192.

Guardiola mexicana Bonpl. 990, 1091, 1138, 5786, 7490, 7497, 7551 . 
Guardiola pappifera P.G. Wilson. 9333, 9533 (TYPUS). Guardiola tulocarpus A. Gray. 9200, 9923, 12326, 12900, 15170, 15791.

Gundlachia triantha (S.F. Blake) Urbatsch \& R.P. Roberts. 24762, 28447, 28533.

Gutierrezia alamanii A. Gray. 1036.

Gutierrezia grandis S.F. Blake. 18364, 18599, 20232, 20263 , 20486, 20728, 21096.

Gutierrezia microcephala (DC.) A. Gray. 16878, 17480, 19328, 19948, 20637, 22361, 23840, 25651, 27824, 28476, 29297.

Gutierrezia sarothrae (Pursh) Britton \& Rusby. 25919, 25973, 27788.

Gutierrezia texana (DC.) Torr. \& A. Gray. 16681, 17680, 17932, 18626, 19076, 19795, 19871, 19947, 20095, 20355, 20607, 21334, 24999, 27067, 27405, 27956, 28043, 28591, 28661, 28762 .

Gymnosperma glutinosum (Spreng.) Less. 16500, 18085, 18431, 19557, 23824, 25066, 25476, 28314, 28478, 28971, 29450, 29454.

Haploesthes fruticosa B.L. Turner. 16555 (TYPUS), 28257, 28388, 28406, 28612, 28627, 28772.

Haploesthes greggii A. Gray. 22330, 25721, 25962, 25987, 27435, 27508, 28450, 28477, 28528, 28604, 29480.

Haploesthes hintoniana B.L. Turner. 29349 (TYPUS), 29358.

Hedosyne ambrosiifolia (A. Gray) Strother. 28864.

Helenium elegans DC. 13625, 16800, 19944, 21232, 29234.

Helenium mexicanum Kunth. 5448, 5656, 13773, 26526.

Helenium microcephalum DC. 24106, 24172.

Helenium scorzonerifolium (DC.) A. Gray. 4403, 15016.

Helianthella gypsophila B.L. Turner. 17571, 18173, 18212, 18246, 18673, 18727, 20426, 20513, 20828, 20833, 20956, 21367, 24485, 25376, 25503, 28031, 28049, 28913.

Helianthella quinquenervis (Hook.) A. Gray. 18852, 18875.

Helianthus annuus L. 19478, 19931, 24169.

Helianthus laciniatus A. Gray. 17124, 19413, 19675, 27657.

Heliomeris multiflora Nutt. 20902, 23930.

Heliopsis аппиа Hemsl. 19530, 19625, 19642, 20602, 27594.

Heliopsis buphthalmoides (Jacq.) Dunal. 9707, 10680, 14736, 14749, 14876, 15058, 26001, 26107, 26176, 26702, 26775.

Heliopsis parviceps S.F. Blake. 6336, 8096 (Type of Heliopsis purpurea Paul G. Wilson), 8166.

Heliopsis parvifolia A. Gray. 19485, 20710, 24865, 24935, 25909, 27088.

Heliopsis procumbens Hemsl. 7970.

Heterosperma pinnatum Cav. 29720.

Heterosperma pinnatum Cav. 1982, 4828, 4870, 6524, 6550, 21120, 27413.

Heterotheca gypsophila B.L. Turner. 18471, 18476, 18634, 22864, 28909.

Heterotheca inuloides Cass. 576, 847, 13024, 15477, 18061

Heterotheca leptoglossa DC. 20904.

Heterotheca mucronata V.L. Harms. 18307, 19139, 19244,
19609, 19667, 19833, 19920, 19993, 20245, 22074, 22297 , 22948, 23286, 24484, 25511.

Heterotheca subaxillaris (Lam.) Britton \& Rusby. 16660, 18528, 18555, 18646, 19303, 19574, 19782, 19797, 19813, 19849, 19869, 20096, 20640, 22264, 22366, 24426, 24574 , 24636, 24986, 27898, 27928, 28231, 28284, 28751.

Hieracium abscissum Less. 1320, 5345, 8999, 15088, 21633, 22295, 22433, 23203, 26086, 26602, 26832, 26833.

Hieracium crepidispermum Fr. 1321, 25569, 25600.

Hieracium dysonymum S.F. Blake. 20275, 24386.

Hieracium gronovii L. 26137, 26446.

Hieracium gypsophilum B.L. Turner. 18238, 18300, 19905, 20239, 22112, 22615, 25491, 25844, 29185, 29204.

Hieracium hintonii Beaman. 7373 (TYPUS).

Hieracium macdonaldii Beaman \& B.L. Turner. 26518, 26611.

Hieracium mexicanum Less. 18315, 18317.

Hofmeisteria dissecta (Hook. \& Arn.) R.M. King \& H. Rob. 3127, 5453, 12292.

Hofmeisteria urenifolia (Hook. \& Arn.) Walp. 10143, 14178.

Hymenopappus flavomarginatus I.M. Johnst. 18439, 18505, 27875, 28930.

Hymenopappus hintoniorum B.L. Turner. 18876 (TYPUS), 18918, 18931.

Hymenostephium cordatum (Hook. \& Arn.) S.F. Blake. 526, $8410,8567,10611,12841,12941,12967,13616,14128$, 14182, 21687, 26338, 28854.

Hymenostephium tenuis (A. Gray) E.E. Schill. \& Panero. 2555, 5489, 9795, 12647.

Hymenostephium uniseriatum E.E. Schill. \& Panero. 2511, 5208, 5349.

Hymenothrix greenmanii (Heiser) B.L. Turner. 4507 (TYPUS).

Hymenoxys insignis (A. Gray ex S. Watson) Cockerell. 17025, 17255, 17767, 17894, 18863, 19360, 19840, 25339, 27534.

Hymenoxys odorata DC. 16762, 17139, 18420, 19504, 27583.

Hymenoxys pinetorum (Standl.) Bierner. 17149, 17294, 25480.

Iostephane heterophylla (Cav.) Hemsl. 8309, 29705.

Iostephane heterophylla (Cav.) Hemsl. 4637, 7922bis, 14422, 17416 bis, 23457.

Iostephane trilobata Hemsl. 4965, 26085, 26170.

Isocoma gypsophila B.L. Turner. 29877.

Isocoma veneta (Kunth) Greene. 25614.

Isocoma coronopifolia (A. Gray) Greene. 29269.

Isocoma gypsophila B.L. Turner. 23034, 25830, 25984, 27097, 27567, 28599, 28807.

Isocoma hartwegii (A. Gray) Greene. 27108.

Isocoma veneta (Kunth) Greene. 17846, 18071, 18560, 19525, 22341, 27474, 28598.

Jaegeria hirta (Lag.) Less. 15530.

Jaegeria hirta (Lag.) Less. 128, 1910, 2718, 5289, 5612, 12400, 12432, 12868, 15342, 17444bis, 26283.

Jaegeria macrocephala Less. 677, 12399, 15364.

Jefea brevifolia (A. Gray) Strother. 16572, 20848, 23020, 
25908, 28394.

Jefea gnaphalioides (A. Gray) Strother. 27695.

Jefea lantanifolia (S. Schauer) Strother. 20561, 22141, 23400, 23644.

Jungia pringlei Greenm. 12879.

Koanophyllon albicaule (Sch. Bip. ex Klatt) R.M. King \& H. Rob. 7592 (Type of Koanophyllon guerreroana B.L. Turner).

Koanophyllon galeana (B.L. Turner) B.L. Turner. 21396, 21512 (TYPUS).

Koanophyllon hintoniorum (B.L. Turner) Velazco. 20037 (TYPUS), 21399, 21657, 21745, 21749.

Koanophyllon longifolium (B.L. Rob.) R.M. King \& H. Rob. 25012, 25016, 28566.

Koanophyllon monanthum (Sch. Bip.) T.J. Ayers \& B.L. Turner. 3185, 10019, 13655, 15806.

Koanophyllon reyrobinsonii B.L. Turner. 20048, 23662 , 23906, 24878.

Koanophyllon richardsonii B.L. Turner. 25059, 25065, 25107, 28156, 28168.

Koanophyllon solidaginoides (Kunth) R.M. King \& H. Rob. 2965.

Lactuca graminifolia Michx. 19143, 22303, 23082, 24637, 28169.

Lactuca ludoviciana (Nutt.) DC. 19669, 20314, 22203.

Lactuca serriola L. 19032, 19542.

Laennecia coulteri (A. Gray) G.L. Nesom. 17142.

Laennecia confusa (Cronquist) G.L. Nesom. 1411.

Laennecia coulteri (A. Gray) G.L. Nesom. 16848, 17848, 18593, 18635, 19473, 19517, 22362, 27613, 28369.

Laennecia gnaphalioides (Kunth) Cass. 2872, 18337.

Laennecia microglossa (S.F. Blake) G.L. Nesom. 21930.

Laennecia schiedeana (Less.) G.L. Nesom. 3044, 20507, 22407, 22516, 23798, 26013, 26065.

Laennecia sophiifolia (Kunth) G.L. Nesom. 1410, 23270.

Lagascea heteropappus Hemsl. 13461.

Lagascea rigida (Cav.) Stuessy. 29769.

Lagascea angustifolia DC. 14918.

Lagascea aurea Stuessy. 12830.

Lagascea decipiens Hemsl. 13206, 14827.

Lagascea helianthifolia Kunth. 224, 2485, 2949, 7496, 8344, 8833, 11672, 12689.

Lagascea heteropappus Hemsl. 2421, 8656, 11106.

Lagascea palmeri (B.L. Rob.) B.L. Rob. 12586, 15844.

Lagascea rigida (Cav.) Stuessy. 2589.

Lasianthaea ceanothifolia (Willd.) K.M. Becker. 2880, 8593, 12158, 12445, 12823, 15157.

Lasianthaea crocea (A. Gray) K.M. Becker. 1779, 1936, 2136, 2251, 8486, 9577, 9828, 14835.

Lasianthaea fruticosa (L.) K.M. Becker. 10742.

Lasianthaea helianthoides DC. 1627, 11340, 13288.

Lasianthaea macrocephala (Hook. \& Arn.) K.M. Becker. 12172, 12498, 12854.

Leibnitzia lyrata (D. Don) G.L. Nesom. 23455, 27222.

Lepidaploa canescens (Kunth) H. Rob. 15393, 16268.

Leucactinia bracteata (S. Watson) Rydb. 19014, 19078, 28894.

Liabellum hintoniorum (B.L. Turner) H. Rob. 2038, 8482.
Liabellum palmeri A. Gray. 2037, 3021.

Liatris punctata Hook. 23775.

Lorandersonia baileyi (Wooton \& Standl.) Urbatsch, R.P. Roberts \& Neubig. 28021, 28086, 28102, 28939.

Machaeranthera tanacetifolia (Kunth) Nees. 16697, 16767, 17133, 17834, 18557, 18676, 19511, 20258, 27719.

Melampodium divaricatum (Rich.) DC. 15459.

Melampodium americanum L. 9134, 12871, 13659, 15587.

Melampodium argophyllum (A. Gray ex B.L. Rob.) S.F. Blake. 16563, 23190, 23529, 27074, 28387, 29127.

Melampodium cinereum DC. 27756.

Melampodium dicoelocarpum B.L. Rob. 9714, 14614.

Melampodium divaricatum (Rich.) DC. 1520, 5518, 12176 , 12965, 13583, 14487, 14648.

Melampodium gracile Less. 1661, 2008, 6207, 6338.

Melampodium linearilobum DC. 2513, 6270, 6420, 9113.

Melampodium longifolium Cerv. ex Cav. 19839, 21362.

Melampodium microcephalum Less. 9205, 12510.

Melampodium montanum Benth. 2017, 22144, 22449, 22908, 23089, 23207, 23449, 24819, 26032, 26175, 26189, 26388, 26544, 26677, 27266.

Melampodium perfoliatum (Cav.) Kunth. 1521, 1838, 2480, 5483, 12972, 15466, 20064, 20528, 20898, 21507, 24564.

Melampodium pilosum Stuessy. 6436, 11428 (TYPUS).

Melampodium repens Sessé \& Moc. 4628.

Melampodium sericeum Lag. 6324.

Melampodium tepicense B.L. Rob. 12276.

Melanthera nivea (L.) Small. 7050, 10567, 15848.

Microspermum flaccidum Paul G. Wilson. 7616 (TYPUS).

Microspermum nummulariifolium Lag. 1823, 1859, 2181, 2795, 2882, 8381, 9671, 11281, 14449, 15100.

Microspermum tenue Paul G. Wilson. 9953 (TYPUS).

Mikania micrantha Kunth. 11753.

Mikania cordifolia (L. f.) Willd. 3580, 7599, 13626.

Mikania micrantha Kunth. 23819.

Milleria quinqueflora L. 1797, 4838, 6604, 9509, 10620, 12157, 15184.

Montanoa bipinnatifida (Kunth) K. Koch. 12684.

Montanoa frutescens (Mairet ex DC.) Hemsl. 15517.

Montanoa leucantha (Lag.) S.F. Blake. 29780.

Montanoa tomentosa Cerv. 29773, 29829.

Montanoa andersonii McVaugh. 12794.

Montanoa bipinnatifida (Kunth) K. Koch. 2401, 16242.

Montanoa frutescens (Mairet ex DC.) Hemsl. 1880, 1977, 17402bis.

Montanoa grandiflora Alamán ex DC. 2579, 10962.

Montanoa imbricata V.A. Funk. 14832.

Montanoa karwinskii DC. 9662, 11565, 12473, 14618.

Montanoa leucantha (Lag.) S.F. Blake. 2721, 5146, 13383, 14818, 14852.

Montanoa mollissima Brongn. ex Groenl. 1456, 15332.

Montanoa revealii H. Rob. 15395.

Montanoa tomentosa Cerv. 5144, 13387, 21656, 22464, 23470, 23495, 23631, 23785.

Nahuatlea hypoleuca (DC.) V.A. Funk. 16564, 17820, 18096, 19983, 23888.

Nicolletia edwardsii A. Gray. 23382, 23550, 24766, 28429, $28770,28881$. 
Onoseris onoseroides (Kunth) B.L. Rob. 11752.

Osbertia stolonifera (DC.) Greene. 1037, 14291, 26554, 26576, 26588, 26640, 26798.

Oteiza scandens Panero \& Villaseñor. 26248, 26433, 26512.

Otopappus acuminatus S. Watson. 14566.

Otopappus imbricatus (Sch. Bip.) S.F. Blake. 1937, 6684.

Otopappus koelzii McVaugh. 16046.

Otopappus mexicanus (Rzed.) H. Rob. 11367.

Oxypappus scaber Benth. 12573, 14913.

Packera bellidifolia (Kunth) W.A. Weber \& A. Löve. 1376, 17353, 18245, 18823, 20208.

Packera coahuilensis (Greenm.) C. Jeffrey. 17922, 18469, 18839, 18877, 18879, 19069, 19148, 19158, 19195, 19216, 19327, 19347, 19807, 20206, 23215.

Packera hintoniorum (B.L. Turner) C. Jeffrey. 17048 (TYPUS), 20188, 21917, 25338, 25494.

Packera loratifolia (Greenm.) W.A. Weber \& A. Löve. 17026, 17204, 17256, 17918, 18297, 18308, 18874, 18896, 19398, 20267, 20443, 21004, 22492.

Packera montereyana (S. Watson) C. Jeffrey. 21085.

Packera sanguisorbae (DC.) C. Jeffrey. 498, 7690, 11927 , 15008, 15705.

Packera tampicana (DC.) C. Jeffrey. 17867.

Packera toluccana (DC.) W.A. Weber \& A. Löve. 491, 3279, 5783, 7482, 8905, 17338, 17369, 19326, 22684.

Packera zimapanica (Hemsl.) C.C. Freeman \& T.M. Barkley. 17411, 17502, 17502bis, 18291, 18306, 18866, 18921, 22428.

Palafoxia texana DC. 16873, 27498.

Parthenium incanum Kunth. 18033.

Parthenium argentatum A. Gray. 19731, 23000, 29313.

Parthenium bipinnatifidum (Ortega) Rollins. 17469, 18556, 20063, 20630, 21909, 25622, 27587, 27591, 27602, 29314.

Parthenium confertum A. Gray. 16779, 19427, 19448, 19488, 19665, 21378, 21554, 24868, 29315.

Parthenium fruticosum Less. 16840.

Parthenium hysterophorus L. 12031, 12225, 12825, 13619, 15177, 16573, 24153, 27955, 28040, 28250, 28282, 29182.

Parthenium incanum Kunth. 16574, 16673, 16718, 16719, 16720, 16721, 16722, 16790, 18097, 20791, 23357, 25164, 25656, 28281, 28455, 28654, 28686, 28850, 29316, 29337, 29455.

Pectis angustifolia Torr. 16580, 21101, 23553, 24725, 27742 , 28430, 28491, 28503, 28766, 28880, 29322.

Pectis canescens Kunth. 5944, 6472.

Pectis decemcarinata McVaugh. 12090.

Pectis haenkeana (DC.) Sch. Bip. 5708.

Pectis holochaeta (S.F. Blake) D.J. Keil. 9519.

Pectis leavenworthii Standl. 15173.

Pectis liebmannii Sch. Bip. ex Hemsl. 26470.

Pectis linearis La Llave. 4815, 6521, 6677.

Pectis linifolia L. 6606 (Type of Pectis linifolia var. hirtella S.F. Blake).

Pectis multiflosculosa (DC.) Sch. Bip. 26494.

Pectis pringlei Fernald. 28824.

Pectis propetes Greenm. 2509, 4797 (Type of Pectis propetes var. holochaeta S.F. Blake), 6422.
Pectis prostrata Cav. 1459, 2978.

Pectis uniaristata DC. 1270, 9715, 10622, 15215.

Perymenium buphthalmoides DC. 973, 13041, 13925, 13997.

Perymenium ghiesbreghtii B.L. Rob. \& Greenm. 2573.

Perymenium globosum B.L. Rob. 7742, 9872, 11404.

Perymenium hintonii McVaugh. 8534, 12045 (TYPUS).

Perymenium hintoniorum B.L. Turner. 20788 (TYPUS), 21499, 23596, 23645, 23733, 23749 (Type of Perymenium hintoniorum var. gypsophilum B.L. Turner), 23855, 25684, 28576.

Perymenium oxycarphum S.F. Blake. 9296.

Perymenium reticulatum J.J. Fay. 7924, 7985 (TYPUS), 8137.

Perymenium tamaulipense B.L. Turner. 22244, 24676, 24821, 24903, 24912.

Peteravenia malvifolia (DC.) R.M. King \& H. Rob. 17702, 24405, 27816, 28189, 28564.

Peteravenia schultzii (Schnittsp.) R.M. King \& H. Rob. 25005.

Pinaropappus roseus (Less.) Less. 29768.

Pinaropappus roseus (Less.) Less. 385, 618, 4678, 11962, 13571, 17763, 18534, 18636, 19013, 19792, 20129, 21972 , 23628, 25392, 27412, 28952, 29096, 29441.

Piqueria pilosa Kunth. 15525.

Piqueria pilosa Kunth. 1306, 2098, 3517 (Type of Piqueria hintonii R.M. King), 26688.

Piqueria trinervia Cav. 135, 136, 2107, 2689, 11902, 12430, 15275, 20886, 21671, 24653, 25021, 25078, 26008.

Pittocaulon bombycophole (Bullock) H. Rob. \& Brettell. 5898, 7790 (TYPUS), 8124.

Pittocaulon hintonii H. Rob. \& Brettell. 15770 (TYPUS).

Pittocaulon praecox (Cav.) H. Rob. \& Brettell. 467, 3206, 3380, 3665.

Plateilema palmeri (A. Gray) Cockerell. 25344, 27195, 29389.

Plectocephalus americanus (Nutt.) D. Don. 17532bis, 17532, 19955, 20018, 24438.

Pluchea carolinensis (Jacq.) G. Don. 17832, 19965, 21841, 21865, 22374.

Pluchea odorata (L.) Cass. 20541.

Pluchea salicifolia (Mill.) S.F. Blake. 3025, 3315, 5640, 12961, 21848, 22085.

Podachaenium eminens (Lag.) Sch. Bip. 12943, 13627.

Porophyllum amplexicaule Engelm. ex A. Gray. 20767, 22155, 22702, 22799, 23046, 25653, 27112, 28295, 28392, 29243.

Porophyllum coloratum (Kunth) DC. 19765, 20485, 20719, 22576, 23590, 23990.

Porophyllum filiforme Rydb. 18219, 19729, 20959, 22712, 22777, 23047, 23765, 24492, 27625, 28032, 28580, 28698. Porophyllum macrocephalum DC. 2036, 6643, 27818.

Porophyllum pringlei B.L. Rob. 2601.

Porophyllum punctatum (Mill.) S.F. Blake. 10464.

Porophyllum scoparium A. Gray. 16594, 16631, 17825, 19084, 19275, 20704, 21778, 21995, 22166, 22792, 22880, 23197, 23375, 23585, 24989, 25475, 28375, 28458, 28504, 28682, 28795, 28857, 29271, 29307, 29369, 29417, 29422, 
29432, 29570.

Porophyllum viridiflorum (Kunth) DC. 2241, 2350, 3050, 5315, 12568, 13388, 15389, 17515bis.

Porophyllum warnockii R.R. Johnson. 8469 (TYPUS).

Psacaliopsis macdonaldii (B.L. Turner) C. Jeffrey. 26794.

Psacalium peltatum (Kunth) Cass. 17413, 27223.

Psacalium amplifolium (DC.) H. Rob. \& Brettell. 2322, 8225, 8349, 13436.

Psacalium amplum (Rydb.) H. Rob. \& Brettell. 8636.

Psacalium brachycomum (S.F. Blake) H. Rob. \& Brettell. 12146, 15288, 15624.

Psacalium cirsiifolium (Zucc.) H. Rob. \& Brettell. 1650, 1678, 8211, 9436, 26714.

Psacalium goldsmithii (B.L. Rob.) H. Rob. \& Brettell. 7984, 8054, 9202.

Psacalium hintoniorum B.L. Turner. 26557 (TYPUS), 26717, 26758.

Psacalium laxiflorum Benth. 968, 7997, 8108.

Psacalium megaphyllum (B.L. Rob. \& Greenm.) Rydb. 2200, 2355, 13480, 15629.

Psacalium nanum Pippen. 14464 (TYPUS).

Psacalium nelsonii Rydb. 26198.

Psacalium peltatum (Kunth) Cass. 1908, 14877, 17406, 20979, 23097, 23218, 23435, 23523, 25535, 26156.

Psacalium peltigerum (B.L. Rob. \& Seaton) Rydb. 7966 (Type of Psacalium peltigerum var. hintonii R.W. Pippen).

Psacalium radulifolium (Kunth) H. Rob. \& Brettell. 17582, 22891, 22935, 23445, 27220.

Psacalium silphiifolium (B.L. Rob. \& Greenm.) H. Rob. \& Brettell. 15078.

Psathyrotopsis hintoniorum B.L. Turner. 22692 (TYPUS), 23191, 23358, 23559.

Pseudelephantopus spicatus (Juss. ex Aubl.) C.F. Baker. 12517.

Pseudelephantopus spicatus (Juss. ex Aubl.) C.F. Baker. 2479, 14990.

Pseudognaphalium attenuatum (DC.) Anderb. 2378, 5426, 9845, 11656.

Pseudognaphalium brachypterum (DC.) Anderb. 19930, 20027, 20749, 20761, 20787, 21237, 21315, 25473, 25921, 25945.

Pseudognaphalium canescens (DC.) Anderb. 18598, 21510, 21530, 21658, 28290.

Pseudognaphalium conoideum (Kunth) Anderb. 18535.

Pseudognaphalium chartaceum (Greenm.) Anderb. 191, 2894, 13517.

Pseudognaphalium elegans (Kunth) Kartesz. 25982.

Pseudognaphalium hintoniorum (G.L. Nesom) Hinojosa \& Villaseñor. 17263 (TYPUS), 17917, 18312, 18902 (Type of Gnaphalium flavocephalum G.L. Nesom), 20742, 22399.

Pseudognaphalium luteoalbum (L.) Hilliard \& B.L. Burtt. 29280.

Pseudognaphalium oxyphyllum (DC.) Kirp. 372, 19828, 25551, 25594, 25596, 26012, 26674, 26740, 26750.

Pseudognaphalium roseum (Kunth) Anderb. 2427, 18177, 19019, 19798, 20703, 22267, 22365, 22470, 25564, 25595, 25847, 26247, 26317, 26400, 27709, 28039.

Pseudognaphalium semiamplexicaule (DC.) Anderb.
12885.

Pseudognaphalium semilanatum (DC.) Anderb. 19917, 19923, 19995, 20709, 21523, 25626.

Pseudognaphalium viscosum (Kunth) Anderb. 803, 3043, 6356, 19522, 22093, 22243, 27664.

Psilactis asteroides A. Gray. 20402, 20466, 25553.

Psilactis brevilingulata Sch. Bip. ex Hemsl. 18624, 27586, 28597.

Psilactis tenuis S. Watson. 19080, 27227.

Psilostrophe gnaphalodes DC. 16538, 16679, 18045, 18234, 18550, 19304, 19476, 19631, 22326, 23341, 24729, 24856, 25990, 27622, 28004, 28405, 28499, 29250.

Pyrrhopappus pauciflorus (D. Don) DC. 17798, 20907, 21372, 21647, 24107, 24591, 24596, 24698, 25117.

Ratibida columnifera (Nutt.) Wooton \& Standl. 16648, 17122, 19437, 19541, 20013, 21437, 27239, 27575, 27600, 28806.

Roldana angulifolia (DC.) H. Rob. \& Brettell. 8818.

Roldana hintonii H. Rob. \& Brettell. 8966.

Roldana albonervia (Greenm.) H. Rob. \& Brettell. 390.

Roldana angulifolia (DC.) H. Rob. \& Brettell. 3061, 15708.

Roldana aschenborniana (S. Schauer) H. Rob. \& Brettell. 8971, 17498, 17516, 19270, 19320, 20899, 22205, 24011, 25118.

Roldana barba-johannis (DC.) H. Rob. \& Brettell. 2365, 2741, 10170, 15731, 15754, 26223.

Roldana candicans (Née) Villaseñor, S. Valencia \& Coombes. 3046, 12774, 13476, 26132, 26157, 26302, 26444.

Roldana cordovensis (Hemsl.) H. Rob. \& Brettell. 15777bis.

Roldana glinophylla H. Rob. \& Brettell. 12220.

Roldana hederifolia (Hemsl.) H. Rob. \& Brettell. 3437, 17534bis.

Roldana hintonii H. Rob. \& Brettell. 8949, 8967 (TYPUS). Roldana lobata La Llave. 2859, 11698, 17489bis, 29819.

Roldana michoacana (B.L. Rob.) H. Rob. \& Brettell. 2755, 4993, 12718, 13469.

Roldana nesomiorum (B.L. Turner) C. Jeffrey. 22585, 27262.

Roldana oaxacana (Hemsl.) H. Rob. \& Brettell. 247, 26069, 26178, 26229, 26230, 26704.

Roldana pennellii H. Rob. \& Brettell. 21261, 27232, 27265.

Roldana platanifolia (Benth.) H. Rob. \& Brettell. 21294.

Roldana reticulata (DC.) H. Rob. \& Brettell. 2747, 8337, 11136, 15595.

Roldana sessilifolia (Hook. \& Arn.) H. Rob. \& Brettell. 29783.

Roldana subcymosa H. Rob. 26154.

Roldana suffulta (Greenm.) H. Rob. \& Brettell. 13455, 17420bis.

Roldana sundbergii (B.L. Turner) B.L. Turner. 21435, 23795, 23812.

Rumfordia floribunda DC. 11134.

Rumfordia alcortae Rzed. 17464, 20890, 22443.

Rumfordia exauriculata B.L. Turner. 19263, 21639, 22501, 27254, 27255. 
Rumfordia floribunda DC. 7612, 14890, 17487bis, 26016, 26241, 26632.

Sabazia humilis (Kunth) Cass. 1895.

Sabazia leavenworthii Longpre. 15526.

Sabazia microspermoides Longpre. 14788 (TYPUS).

Sabazia multiradiata (Seaton) Longpre. 2770, 14898 (Type of Sabazia liebmannii var. hintonii Longpre), 26030, 26033, 26047, 26073, 26082, 26384, 26655, 26703, 26791.

Salmea oligocephala Hemsl. 1490.

Salmea scandens (L.) DC. 7572, 14978.

Sanrobertia gypsophila (B.L. Turner) G.L. Nesom. 25991, 27098, 27655, 28732, 29881.

Sanvitalia angustifolia Engelm. ex A. Gray. 19628.

Sanvitalia ocymoides DC. 19065, 21425, 28014, 28964.

Sanvitalia procumbens Lam. 1201, 5442, 13408.

Sartwellia puberula Rydb. 29567.

Sartwellia gypsophila A.M. Powell \& B.L. Turner. 29274.

Sartwellia mexicana A. Gray. 17835, 19446, 19514, 20464, 22363, 27717, 27722, 28048, 28596, 28674, 28760.

Sartwellia puberula Rydb. 28830, 28878, 29306, 29319, 29398, 29423.

Sclerocarpus divaricatus (Benth.) Hemsl. 12091, 13658, 15139, 15165, 26456.

Sclerocarpus multifidus Greenm. 4573, 6425, 8150, 9187, 9419.

Sclerocarpus uniserialis (Hook.) Hemsl. 5441, 5504, 5550, 13144, 17476, 18625, 19055, 19176, 20400, 20523, 20829, 21311, 21490, 22551, 22582, 23688, 23895, 23984, 25032, 29041.

Schkuhria pinnata (Lam.) Kuntze ex Thell. 9544, 10696, 29756.

Schkuhria pinnata (Lam.) Kuntze ex Thell. 1405, 6228, 25573.

Selloa plantaginea Kunth. 4613.

Senecio bracteatus Klatt. 26224, 26367, 26441, 26539, 26555, 26649, 26698, 26816.

Senecio callosus Sch. Bip. 13473, 13478, 14959, 26164, 26303.

Senecio carnerensis Greenm. 17290, 17875, 17914, 17974 , 18292, 18316, 18357, 18895, 20436, 20730.

Senecio cinerarioides Kunth. 496, 3278, 3472, 8822.

Senecio flaccidus Less. 22850, 23527.

Senecio lithophilus Greenm. 24304, 24673, 25268.

Senecio madrensis A. Gray. 17297, 17410, 17911, 18281, 18304, 18906, 20316, 20441, 20740, 21332, 22404, 22420, 23967, 25552, 25599.

Senecio multidentatus Sch. Bip. ex Hemsl. 1042.

Senecio picridis S. Schauer. 8972, 23799, 23804, 23926, 25068, 25075, 28166, 28210.

Senecio platypus Greenm. 16928, 19175, 19199.

Senecio polypodioides (Greene) T. Durand \& B.D. Jacks. 11229 .

Senecio powellii B.L. Turner. 18285, 27027, 28757.

Senecio richardsonii B.L. Turner. 19028, 20444, 21308, 21318, 22910, 22993, 23214.

Senecio roseus Sch. Bip. 26211.

Senecio runcinatus Less. 555, 3060, 5766.

Senecio stoechadiformis DC. 8626, 11836.
Senecio subauriculatus Greenm. 26152.

Senecio viejoanus B.L. Turner. 22394, 23313, 23969 (TYPUS).

Senecio vulgaris L. 4898, 8327, 17984, 18153.

Sidneya tenuifolia (A. Gray) E.E. Schill. \& Panero. 29575.

Sidneya tenuifolia (A. Gray) E.E. Schill. \& Panero. 16551, 16584, 16633, 18044, 18432, 18488, 18569, 19475, 19477, 19722, 19847, 21981, 22005, 22998, 23538, 27736, 27745, 28391, 28461, 28773, 29284.

Sigesbeckia agrestis Poepp. 2792, 2929, 5256, 5310, 12431.

Sigesbeckia jorullensis Kunth. 1904, 2862, 3047, 5148, 9868, 11293, 12907, 13232, 14745, 14805, 15694, 17445bis, 26034, 26044, 26074, 26208, 26285, 26708, 26771.

Sigesbeckia repens B.L. Rob. \& Greenm. 26080, 26188, 26540, 26743.

Simsia amplexicaulis (Cav.) Pers. 15494, 18783, 19035, 19573, 19673, 19874, 20478, 20479, 20881, 25578.

Simsia annectens S.F. Blake. 2693 (Type of Simsia subsetosa H. Rob. \& Brettell), 11271, 11651, 12714, 12855, 14976 (Type of Simsia hintonii H. Rob. \& Brettell).

Simsia calva (A. Gray \& Engelm.) A. Gray. 17475, 17681, 18627, 19054, 20573, 20747, 20748, 21038, 21170, 21253 , 23660, 25621, 28011, 28958.

Simsia eurylepis S.F. Blake. 28197.

Simsia foetida (Cav.) S.F. Blake. 1741, 2216, 2801, 2935, 5538, 8582, 9625, 13289.

Simsia lagasciformis DC. 5549, 8578, 28818.

Simsia sanguinea A. Gray. 1874, 6706, 10647 (Type of Cosmos hintonii Sherff), 13320.

Sinclairia glabra (Hemsl.) Rydb. 2399, 2627, 11682, 12678, 13401, 16161 (Type of Sinclairia glabra var. minor B.L. Turner).

Smallanthus maculatus (Cav.) H. Rob. 1347, 5227, 16816, 19038, 19061, 19186, 22483, 24443, 24556, 24705, 26315, 27699, 27853.

Solidago missouriensis Nutt. 18780.

Solidago ericamerioides G.L. Nesom. 18763 (TYPUS), 28226.

Solidago gigantea Aiton. 19935, 20339, 23813.

Solidago hintoniorum G.L. Nesom. 17264 (TYPUS), 17884, 17925, 18335, 18613, 20508, 20867, 21492, 22053, 22444, 22547, 23323, 25886, 27918, 27924, 28042, 28069, 28075, 28081, 28085, 28093, 28103.

Solidago missouriensis Nutt. 19300, 21858, 22721.

Solidago orientalis (G.L. Nesom) G.L. Nesom. 19257, 21262, 21617, 21645, 21684, 27489, 28573.

Solidago simplex Kunth. 22120, 22298.

Solidago velutina DC. 16605, 17123, 20463, 20469, 25430, 27414, 27574, 27856, 28279, 28283.

Solidago wrightii (G.L. Nesom) G.L. Nesom. 18333 (TYPUS), A. Gray. 25813.

Sonchus asper (L.) Hill. 460.

Sonchus asper (L.) Hill. 8247.

Sonchus oleraceus L. 6185, 7567, 12986, 18151, 19434 , 19483, 19521, 19632, 19633, 23921, 23963.

Sonchus tenerrimus L. 21920.

Stephanomeria pauciflora (Torr.) A. Nelson. 27509. 
Stevia salicifolia Cav. 29789.

Stevia tomentosa Kunth. 18016, 29808.

Stevia triflora DC. 14771.

Stevia aschenborniana Sch. Bip. 2660, 2728, 3094, 9733, 13380, 17500bis.

Stevia berlandieri A. Gray. 19005, 19051, 19803, 20353, 20570, 21178, 21197, 21593, 21651, 21667, 21741, 22054, 22058, 22101, 22247, 22902, 23138, 24162, 24475.

Stevia caracasana DC. 2709, 11304, 12857, 12916.

Stevia connata Lag. 9467.

Stevia deltoidea Greene. 2392, 13419.

Stevia elatior Kunth. 112, 131, 1326, 12909, 15284, 17470bis, 26517.

Stevia hintonii (Grashoff) B.L. Turner. 4537 (TYPUS), 14505.

Stevia hintoniorum B.L. Turner. 21611 (TYPUS), 21626, 21664, 22108, 22219, 22413, 23428, 23429, 23914, 24920, 24930, 24931, 27490.

Stevia hypomalaca B.L. Rob. 2462, 2462bis.

Stevia incognita Grashoff. 21702, 21710, 22471, 22485, 22512, 22584, 23788, 23810, 26015, 26078, 26120, 27276, 27278, 27280, 27289.

Stevia jorullensis Kunth. 8388, 13475, 17118, 19073, 19919 , 22521, 22552, 22583.

Stevia latifolia Benth. 14957.

Stevia micrantha Lag. 20871.

Stevia microchaeta Sch. Bip. 26027, 26255, 26353, 26449, 26570.

Stevia monardifolia Kunth. 11115.

Stevia origanoides Kunth. 17427, 20897.

Stevia ovata Willd. 1905, 2105, 2205, 2277, 2876bis, 2974, 14671, 15268, 15567, 17423bis, 17423, 19991, 20030, 20873, 20905, 21609, 21628, 21650, 21670, 21691, 21700, 22130, 22474, 22498, 22508, 22518, 23588, 23681, 23786, 23796, 23944, 25630, 27961, 27984, 27986, 27997, 28045, 28046, 28200, 28211.

Stevia pilosa Lag. 17409, 18912, 19816, 19836, 20511, 20581, 22209, 22236, 23281, 24573, 29172.

Stevia quiexobra B.L. Turner. 26141 (TYPUS).

Stevia salicifolia Cav. 1452, 16845, 18460, 18591, 19845 , 19909, 20075, 20149, 20213, 20497, 21504, 21592, 22059, 22424, 22497, 24933, 25069, 25119, 28212.

Stevia seemannii Sch. Bip. 12816, 12888, 15586.

Stevia serrata Cav. 1399, 7932, 12423, 15261, 15438, 19248, 21243, 21340, 21601, 22606, 23234, 23924, 24537, 25592.

Stevia suaveolens Lag. 11135, 17456bis.

Stevia subpubescens Lag. 3149, 17501bis, 26231, 26259, 26260, 26307, 26395, 26443, 26618.

Stevia tephra B.L. Rob. 17426, 19262, 22495, 24862, 24863.

Stevia tomentosa Kunth. 18592, 19815, 20706, 21637, 23779, 23780, 27253.

Stevia trifida Lag. 434, 2805, 2863, 2957, 11329, 12902.

Stevia viscida Kunth. 2125, 2155, 8641, 9735, 13242, 22127.

Steviopsis adenosperma (Sch. Bip.) B.L. Turner. 1907, 3110 , 11310, 17526bis.

Steviopsis nesomii B.L. Turner. 22447, 23947, 23958, 24031,
27705.

Strotheria gypsophila B.L. Turner. 25995, 27878, 28592, 28752.

Symphyotrichum carnerosanum (S. Watson) G.L. Nesom. 16918, 17247, 17872, 18324, 18354, 18619, 19052, 19173, 19829, 21250, 21431, 21698, 23909, 23915, 24009, 24941, 27482, 27935.

Symphyotrichum expansum (Poepp. ex Spreng.) G.L. Nesom. 917, 2035, 2186, 5855, 6584, 11994, 12424, 12809, 17689, 19513, 19634, 21481, 21636, 29279.

Symphyotrichum falcatum (Lindl.) G.L. Nesom. 18608.

Symphyotrichum hintonii (G.L. Nesom) G.L. Nesom. 11316 (TYPUS).

Symphyotrichum moranense (Kunth) G.L. Nesom. 2939, 3065, 3230, 8804, 11148.

Symphyotrichum praealtum (Poir.) G.L. Nesom. 18089, 19932.

Synedrella nodiflora (L.) Gaertn. 5362, 5498, 7558, 7581, 10969, 14701.

Tagetes erecta L. 11149, 11516.

Tagetes filifolia Lag. 9545, 11429.

Tagetes foetidissima DC. 11114.

Tagetes micrantha Cav. 29717.

Tagetes subulata Cerv. 11536.

Tagetes tenuifolia Cav. 18056.

Tagetes erecta L. 1942, 4954, 4955, 9576, 9776, 13312 , 14663, 15060, 15334, 15578.

Tagetes filifolia Lag. 1577, 1651, 15211, 26346.

Tagetes foetidissima DC. 2116, 2909, 3056, 12376, 15565.

Tagetes lemmonii A. Gray. 5849.

Tagetes lucida Cav. 1272, 12422, 15134, 15379, 15450, 17878, 18325, 18578, 18617, 19806, 20901, 21324, 24567, 25539, 26445, 26718, 29189.

Tagetes parryi A. Gray. 20894.

Tagetes stenophylla B.L. Rob. 2338, 2464, 9649, 14910.

Tagetes subulata Cerv. 1975, 2339, 5264, 12889, 14914.

Tagetes tenuifolia Cav. 2062, 2090, 2263, 2514.

Tamaulipa azurea (DC.) R.M. King \& H. Rob. 19278, 19960, 19977, 21735, 21781, 21790, 21829, 21836, 22719, 23879, 24977.

Tanacetum parthenium (L.) Sch. Bip. 22246, 24329.

Taraxacum officinale F.H. Wigg. 1046, 8998, 19363, 19410.

Telanthophora andrieuxii (DC.) H. Rob. \& Brettell. 3267, 3284, 8932, 8996.

Tetrachyron grayi (Klatt) Wussow \& Urbatsch. 17558, 23106.

Tetraneuris linearifolia (Hook.) Greene. 17762, 18244, 18404, 18418, 18620, 18629, 18654, 18675, 19842, 20954, 22023, 22055, 24335, 25544, 27942, 27943, 28083, 29001, 29020.

Tetraneuris scaposa (DC.) Greene. 16835, 18252, 20223, 21903, 22775, 25773, 25925, 25980, 28005, 28343.

Thelesperma longipes A. Gray. 29884.

Thelesperma filifolium (Hook.) A. Gray. 20945, 23130.

Thelesperma graminiformis (Sherff) Melchert. 17346.

Thelesperma longipes A. Gray. 16553, 18518, 18983, 20393, 21979, 22269, 22315, 22348, 25505, 25625, 25720, 25937, 
28723, 29341.

Thelesperma megapotamicum (Spreng.) Kuntze. 20250, 22852, 23196, 25456, 27947, 28385, 28792.

Thelesperma muelleri (Sherff) Melchert. 17205.

Thelesperma scabridulum S.F. Blake. 19533, 22342, 27651, 28607.

Thelesperma simplicifolium A. Gray. 18728, 19012, 20404, 20516, 21090, 21494, 21759, 22039, 22347, 23111, 23170, 23736, 23864, 29180.

Thelesperma subaequale S.F. Blake. 25947.

Thymophylla acerosa (DC.) Strother. 19280, 19855, 20606, 21771, 25681, 28217, 28393, 28403, 28454, 28529, 28535, 28594, 28803, 29287, 29425.

Thymophylla gypsophila (B.L. Turner) Strother. 29360.

Thymophylla micropoides (DC.) Strother. 24265, 28247, 28785.

Thymophylla pentachaeta (DC.) Small. 16634, 16945, 17309, $18455,18525,19230,19606,19945,22783,23526,24348$, 24888, 27077, 28007, 29276, 29399, 29576.

Thymophylla setifolia Lag. 18086, 18175, 18658, 19247, 19310, 19608, 21582, 21583, 21772, 28009.

Thymophylla tenuifolia (Cass.) Rydb. 19594, 19748, 19852 , 23030, 25986, 27589, 28665, 28676, 28691.

Tithonia tubiformis (Jacq.) Cass. 29820.

Tithonia diversifolia (Hemsl.) A. Gray. 2293, 8484, 26308, 26323, 26460.

Tithonia rotundifolia (Mill.) S.F. Blake. 1992, 6647, 11469, 12450, 14702, 15308.

Tithonia tubiformis (Jacq.) Cass. 2187, 2356, 9874, 11547, 12358, 12875, 13618, 15581, 18644, 18651, 19724, 20019.

Townsendia mexicana A. Gray. 17318.

Townsendia mexicana A. Gray. 18834, 19224, 19749, 20107, 20620, 27030, 27592, 28288, 28651, 28750, 29092.

Tragopogon porrifolius L. 25416.

Tridax bicolor A. Gray. 4780, 6271.

Tridax bilabiata A.M. Powell. 6449 (TYPUS).

Tridax brachylepis Hemsl. 2363, 2568, 8687.

Tridax coronopifolia (Kunth) Hemsl. 1466, 5435, 5553, 6032, 6283, 24277.

Tridax hintoniorum B.L. Turner. 21566, 22031 (TYPUS), 22061, 25378, 25485, 27927.

Tridax platyphylla B.L. Rob. 26469.

Tridax procumbens L. 3000bis, 3009, 13594, 16910, 21769 , 24278, 25478.

Tridax tenuifolia Rose. 15431.

Trigonospermum annuum McVaugh \& Lask. 20870.

Trigonospermum auriculatum B.L. Turner. 10301 (TYPUS).

Trigonospermum hintoniorum B.L. Turner. 23419, 23476, 23478, 23493, 24873 (TYPUS), 25098.

Trigonospermum melampodioides DC. 2432, 3023, 7460, 11915, 12908, 13534, 14189, 14934, 17426bis, 26309.

Trixis alata D. Don. 2198, 8546, 9825.

Trixis angustifolia DC. 22713, 22800.

Trixis californica Kellogg. 19085, 21117, 21984, 22996, 23580, 28526, 28851, 29233.

Trixis hintoniorum B.L. Turner. 26474 (TYPUS).

Trixis inula Crantz. 20021, 20878.
Trixis mexicana Lex. 10393, 11163, 11797, 12601, 12984, 13607.

Trixis michuacana Lex. 2845, 11718, 12896.

Trixis pringlei B.L. Rob. \& Greenm. 2953, 5469.

Varilla mexicana A. Gray. 23546, 27438, 29363.

Verbesina persicifolia DC. 18059.

Verbesina tetraptera (Ortega) A. Gray. 29861.

Verbesina aramberrana B.L. Turner. 21963, 21975, 22359 (TYPUS), 23638, 27062.

Verbesina coahuilensis A. Gray. 19212, 19236, 20422, 21034, 21087, 23669, 24367, 25683.

Verbesina crocata (Cav.) Less. 2476, 5707, 10039.

Verbesina chihuahuensis A. Gray. 22789, 28186.

Verbesina daviesiae B.L. Turner. 18914, 19041, 20319, 20421, 20439, 20484, 20495, 22006.

Verbesina encelioides (Cav.) A. Gray. 17847, 18679, 19100, 19441, 19548, 19569, 22184, 24995, 25876, 27610, 28368. Verbesina fastigiata B.L. Rob. \& Greenm. 2384, 11526, 13507.

Verbesina fayii B.L. Turner. 26028.

Verbesina gigantea Jacq. 1976, 2080.

Verbesina hintoniorum B.L. Turner. 18611, 18756, 18778, 21581, 27012, 27911, 28110, 28133, 28915.

Verbesina hypoglauca Sch. Bip. ex Klatt. 17429, 19264, 21354, 22384, 22503, 23806, 23951, 25074, 27281, 27714.

Verbesina hypomalaca B.L. Rob. \& Greenm. 16811, 18529, 18980, 19615, 19670, 19671, 19784, 20746, 22079, 23075, 25330, 25455, 25536, 27404, 28008, 28993, 29162.

Verbesina jimrobbinsii B.L. Turner. 12580.

Verbesina klattii B.L. Rob. \& Greenm. 2763, 5320, 8793, 8864, 12783, 13522, 17483bis.

Verbesina langlassei B.L. Rob. 9869, 12472.

Verbesina liebmannii Sch. Bip. ex Klatt. 14756.

Verbesina longipes Hemsl. 17842, 18229, 18716, 18732 , 18750, 19589, 19771, 21106, 22030, 22057, 23019, 23625, 25464, 25554, 25893, 27386, 27411.

Verbesina macdonaldii B.L. Turner. 26115, 26117, 26284, 26392.

Verbesina macvaughii B.L. Turner. 26344.

Verbesina mexiae B.L. Turner. 11126.

Verbesina miahuatlana B.L. Turner. 26277, 26294 (TYPUS), 26354.

Verbesina microptera DC. 16900, 17425, 18353, 21168 , 24962, 25008.

Verbesina mollis Kunth. 19201, 20771, 21438, 21655, 27772 , 28127.

Verbesina nana (A. Gray) B.L. Rob. \& Greenm. 27857.

Verbesina oligantha B.L. Rob. 11603, 12679.

Verbesina olsenii B.L. Turner. 18645, 19053, 19520, 21313.

Verbesina oncophora B.L. Rob. \& Seaton. 7467.

Verbesina parviflora (Kunth) S.F. Blake. 15052.

Verbesina persicifolia DC. 20846, 21414, 21673, 22465, 23399, 23443, 23634, 23777, 23880, 24879, 24991, 24997, 25002, 25057, 25130.

Verbesina perymenioides Sch. Bip. ex Klatt. 2425, 2469.

Verbesina pterocaula DC. 1499, 8452.

Verbesina scabrida Rzed. 14670. 
Verbesina sphaerocephala A. Gray. 2526, 8569, 8595. Verbesina stenophylla Greenm. 8432, 9414, 10539.

Verbesina tamaunuevana B.L. Turner. 27252 (TYPUS), 27703.

Verbesina tetraptera (Ortega) A. Gray. 1491, 1518, 2043, 8130, 9395.

Verbesina virgata Cav. 17469bis.

Verbesina zaragozana B.L. Turner. 18093 (Type of Verbesina zaragosana var. intermedia G.S. Hinton \& B.L. Turner), 19243, 20825, 20850, 21762, 23514, 23632, 23702, 25645, 25686, 25726.

Vernonanthura hintoniorum (B.L. Turner) H. Rob. 24525, 24617, 24711 (TYPUS), 24791, 25027.

Vernonanthura liatroides (DC.) H. Rob. 2417, 2724, 9826, 12494.

Vernonanthura patens (Kunth) H. Rob. 427, 13612, 13640, 14009.

Vernonia alamanii DC. 346, 11104, 13541, 14071, 17494bis, 17514bis.

Vernonia greggii A. Gray. 17111, 17400, 18481, 18665, 19321, 19835, 20408, 20545, 20553, 21568, 22078, 22132, 24114, 24117, 24187, 24190, 24512, 24543, 25313, 25433.

Vernonia karvinskiana DC. 13674, 20874, 26331.

Vigethia mexicana (S. Watson) W.A. Weber. 20028, 20360, 24104, 24131, 25323.

Viguiera dentata (Cav.) Spreng. 29858, 29859.

Viguiera dentata (Cav.) Spreng. 121, 7739, 11301, 11665, 17474, 18606, 18643, 19077, 19089, 19237, 19529, 19674, 19772, 19953, 19958, 20641, 20751, 21594, 21717, 21724, 22325, 22329, 22466, 23599, 23653, 23666, 23680, 23729, 24288, 27813, 28154, 28451, 28471, 28872, 29056, 29347, 29400, 29429.

Viguiera potosina S.F. Blake. 17897, 25527, 25819.

Wedelia acapulcensis Kunth. 10571, 17783, 18622, 19250, 20381, 20540, 20563, 20572, 20780, 21045, 21149, 21256, 21312, 21389, 21681, 21925, 22856, 23664, 24359, 24483, 25472, 25514, 25699, 25872, 28386, 28995.

Wedelia hintoniorum B.L. Turner. 1352 (TYPUS).

Wedelia strigosa Hook. \& Arn. 14568.

Xanthisma crutchfieldii (B.L. Turner) D.R. Morgan \& R.L. Hartm. 18253, 19295, 20238, 21898, 27912.

Xanthisma gypsophilum (B.L. Turner) D.R. Morgan \& R.L. Hartm. 28518, 29348, 29407.

Xanthisma pseudorestiforme B.L. Turner. 28398 (TYPUS), 28633.

Xanthisma rhizomatum (M.C. Johnst.) D.R. Morgan \& R.L. Hartm. 19559, 24854, 25983, 27032, 27653.

Xanthisma spinulosum (Pursh) D.R. Morgan \& R.L. Hartm. 16556, 16579, 16646, 17129, 18371, 18434, 18652, 19435, 19474, 19519, 19622, 19879, 20247, 20639, 20955, 21119, 22709, 23194, 23561, 24428, 28337, 28356, 28389, 28446, 28617, 28666, 28817, 28838, 28870, 29115, 29267, 29404. Xanthium strumarium L. 5026bis, 5501, 20753.

Xylovirgata pseudobaccharis (S.F. Blake) Urbatsch \& R.P. Roberts. 16546 (TYPUS), 23193.

Zaluzania megacephala Sch. Bip. 17248, 18581, 18621, 19037, 19062, 19112, 21264, 22491, 23278, 23800, 23920, 27231, 27923, 27983.
Zaluzania mollissima A. Gray. 27001.

Zaluzania parthenioides (DC.) Rzed. 16856, 16887, 17895, 19067, 19657, 19734, 19770, 19862, 19966.

Zaluzania triloba (Ortega) Pers. 17478.

Zandera andersoniae (B.L. Turner) D.L. Schulz. 1925, 11852 (TYPUS).

Zandera blakei (McVaugh \& Lask.) D.L. Schulz. 14706 (TYPUS).

Zinnia acerosa (DC.) A. Gray. 16502, 17907, 18222, 19536, 21787, 21932, 24487, 29143, 29152.

Zinnia americana (Mill.) Olorode \& A.M. Torres. 1259, 1460, 4475, 6435, 6438, 9206, 9716, 15155.

Zinnia angustifolia Kunth. 1633, 2175, 7959, 8182, 11205 , 12164, 15589.

Zinnia anomala A. Gray. 25957, 28658, 29390.

Zinnia elegans Jacq. 1482, 1483, 4432, 4789, 4825, 6712, 6890, 8143, 8536, 9822, 12442, 13087, 14473, 15147.

Zinnia flavicoma (DC.) Olorode \& A.M. Torres. 6316, 8033, 8036, 9139, 9830, 12016, 12038 (Type of Zinnia jugistyla Paul G. Wilson).

Zinnia grandiflora Nutt. 27070.

Zinnia juniperifolia (DC.) A. Gray. 16836, 18113, 18230, 18678, 19088, 19305, 19678, 27069, 28697.

Zinnia peruviana (L.) L. 1676, 1806, 4315, 4450, 4500, 4561, 8168, 12633, 16689, 17596, 20014, 20015, 20097, 20335, 20583, 20887, 23327, 24431, 27267.

Zinnia venusta (A.M. Torres) Olorode \& A.M. Torres. 6450, 8141, 8148 (TYPUS).

Zinnia zinnioides (Kunth) Olorode \& A.M. Torres. 10600.

Family Balsaminaceae

Impatiens balsamina L. 25468.

\section{Family Basellaceae}

Anredera ramosa (Moq.) Eliasson. 13402.

Anredera vesicaria (Lam.) C.F. Gaertn. 5049, 6903, 15237, 17586bis.

\section{Family Begoniaceae}

Begonia gracilis Kunth. 17994.

Begonia asteroides L.B. Sm. \& B.G. Schub. 2206 (TYPUS), 2428.

Begonia balmisiana Ruiz ex Klotzsch. 1008, 1114, 7964, 8058, 13947, 13983, 15074, 17179.

Begonia barkeri Knowles \& Westc. 2766.

Begonia biserrata Lindl. 1005, 1331, 1672, 4896, 7979, 8081bis, 8081, 10593.

Begonia cylindrata L.B. Sm. \& B.G. Schub. 5042 (TYPUS), 8186, 8425, 8471.

Begonia extranea L.B. Sm. \& B.G. Schub. 937, 993 (TYPUS), 7916, 8067, 8077, 9137, 9214, 10380, 10602, 13067, 13976, 15111, 15975.

Begonia fernaldiana L.B. Sm. \& B.G. Schub. 9425 (TYPUS).

Begonia franconis Liebm. 24632.

Begonia gracilis Kunth. 1569, 2005, 2054, 7000, 7020, 7988, 8132 , 9758, 10739, 11257, 15990, 17421bis, 22451, 23223, 23423, 24704, 24895, 25103. 
Begonia hintoniana L.B. Sm. \& B.G. Schub. 2050 (TYPUS), 2284, 6786, 7021, 8558, 9611, 11453.

Begonia michoacana L.B. Sm. \& B.G. Schub. 15186 (TYPUS).

Begonia monophylla A. DC. 4553, 8028, 9117.

Begonia nemoralis L.B. Sm. \& B.G. Schub. 15063 (TYPUS).

Begonia oaxacana A. DC. 26437.

Begonia plebeja Liebm. 2518, 7045, 11474, 16001, 16013, 16020.

Begonia portillana S. Watson. 13948.

Begonia rhodochlamys L.B. Sm. \& B.G. Schub. 15182, 15190 (TYPUS).

Begonia squarrosa Liebm. 7208, 7386, 7436, 8815.

Begonia stigmosa Lindl. 15658.

Begonia uniflora S. Watson. 17670, 20546, 20810, 21475, 21497, 21678, 24917, 28571, 28719.

Begonia uruapensis Sessé \& Moc. 6803, 7022, 12505, 12579 , 15991, 16029, 16055, 16056.

\section{Family Berberidaceae}

Berberis eutriphylla (Fedde) C.H. Müll. 20113, 25804.

Berberis gracilis Benth. 17494, 17495, 17721, 19220, 20137, 25100.

Berberis lanceolata Benth. 4102, 4103bis, 7547.

Berberis moranensis Schult. \& Schult. f. 3262, 3850, 5591, 8915.

Berberis muelleri (I.M. Johnst.) Marroq. ex Laferrière \& Marroq. 24833, 27494, 27641.

Berberis pinnata Lag. 488.

Berberis trifolia (Schltdl. \& Cham.) Schult. \& Schult. f. 8902.

Berberis trifoliolata Moric. 16652, 16743, 18386, 19450, 29293.

\section{Family Betulaceae}

Alnus acuminata Kunth. 29851.

Alnus acuminata Kunth. 6395, 6845, 8785, 10705, 11875 , 14794, 14800, 14928, 15415, 26066, 26095, 26199, 26261, 26391, 26442, 26721, 26782.

Alnus jorullensis Kunth. 3545, 14943, 15406, 15521.

Carpinus tropicalis (Donn. Sm.) Lundell. 3228, 3456, 11817 , 14312, 14329, 25270.

Ostrya virginiana (Mill.) K. Koch. 7938, 10178, 14247, 14264, 22124, 22307, 26415.

\section{Family Bignoniaceae}

Adenocalymma inundatum Mart. ex DC. 3784 (Type of Adenocalymma hintonii Sandwith), 4137, 5655, 6027, 6281, 7252, 7259, 7768, 7854, 7880, 7881.

Amphilophium buccinatorium (DC.) L.G. Lohmann. 286, 3620 .

Amphilophium crucigerum (L.) L.G. Lohmann. 7153, 8025, 8506, 10185, 10580, 13817, 13885, 14253, 24182, 25314.

Amphilophium paniculatum (L.) Kunth. 1190, 4117, 7235, 7289, 8167, 11275, 11546, 13816, 16278.

Astianthus viminalis (Kunth) Baill. 721, 4147, 4565, 9972, 15870 .
Bignonia aequinoctialis L. 10067, 10219.

Bignonia diversifolia Kunth. 14603, 16213.

Crescentia alata Kunth. 755, 1165, 5840, 5941, 6315, 7111.

Crescentia cujete L. 5804, 7112.

Chilopsis linearis (Cav.) Sweet. 16598, 17781.

Dolichandra quadrivalvis (Jacq.) L.G. Lohmann. 10234, 10319, 13765, 13792, 14257.

Dolichandra unguis-cati (L.) L.G. Lohmann. 4285, 7586, 7769, 10236, 13830.

Fridericia patellifera (Schltdl.) L.G. Lohmann. 11443, 11732, 13915.

Godmania aesculifolia (Kunth) Standl. 3912, 5740, 6109, 9092, 9982, 10001.

Handroanthus impetiginosus (Mart. ex DC.) Mattos. 3014, 5301, 7130, 7292, 7407, 13532.

Mansoa verrucifera (Schltdl.) A.H. Gentry. 5832.

Parmentiera aculeata (Kunth) Seem. 5805, 7126, 7128, 7276.

Roseodendron donnell-smithii (Rose) Miranda. 16307.

Tabebuia heterophylla (DC.) Britton. 10022, 10228.

Tecoma stans (L.) Juss ex Kunth. 314, 2483, 2785, 3841, 3977, 4323, 6101, 6491, 10950, 11324, 11548, 12443, 13066, 13432, 14856, 15291, 16142, 16530, 16657, 17463bis, 17823, 19433, 19650, 23549, 24968, 27476.

Xylophragma seemannianum (Kuntze) Sandwith. 15829.

\section{Family Bixaceae}

Amoreuxia palmatifida Moc. \& Sessé ex DC. 739, 4276, 4554, 9057, 9354, 10389.

Amoreuxia wrightii A. Gray. 28376.

Bixa orellana L. 10233, 10770, 11500, 11765, 14130.

Cochlospermum vitifolium (Willd.) Spreng. 3123, 3596, 3914, 5390, 7255, 9811, 9979, 12933, 16273.

\section{Family Boraginaceae}

Antiphytum geoffreyi N. Mend. \& Flores Olv. 29132, 29613 (TYPUS).

Antiphytum heliotropioides A. DC. 29885.

Antiphytum hintoniorum Higgins \& B.L. Turner. 29888, 29894, 29895, 29896.

Antiphytum heliotropioides A. DC. 17691, 18699, 19686, 19786, 20072, 20461, 21037, 21870, 22162, 22785, 23745, 25367, 28012, 28147, 28185.

Antiphytum hintoniorum Higgins \& B.L. Turner. 18098, 18211 (TYPUS), 18321, 18326, 18754, 18784, 19125, 28716.

Antiphytum parryi S. Watson. 28919.

Cryptantha geohintonii B.L. Turner. 29563, 29607.

Cryptantha gypsites I.M. Johnst. 29882, 29883.

Cryptantha albida (Kunth) I.M. Johnst. 16792.

Cryptantha geohintonii B.L. Turner. 28616, 28635 (TYPUS), 28779.

Cryptantha gypsites I.M. Johnst. 17321, 17836, 18180, 18183, 19445, 19546, 27028, 27581, 28605.

Cryptantha mexicana (Brandegee) I.M. Johnst. 25783, 27003, 29088, 29110, 29134, 29145.

Cryptantha palmeri (A. Gray) Payson. 27712. 
Cynoglossum amabile Stapf. \& J.R. Drumm. 11891, 26546, 26648.

Cynoglossum henricksonii L.C. Higgins. 18703, 18717 , 18943, 21938, 24922, 27100.

Hackelia hintoniorum (B.L. Turner) Sutorý. 26063, 26206 (TYPUS), 26652, 26678, 26817.

Hackelia leonotis I.M. Johnst. 17046, 17302, 17501, 18856.

Hackelia mexicana (Schltdl. \& Cham.) I.M. Johnst. 1303, 4901, 14435.

Lithospermum strictum Lehm. 29749.

Lithospermum barbigerum (I.M. Johnst.) J. Cohen. 21351, 22117, 22912, 24906, 25283.

Lithospermum calcicola B.L. Rob. 18251, 18352, 20418, 25432, 25443, 25546, 25582, 25932.

Lithospermum discolor M. Martens \& Galeotti. 1373, 1436, 1951, 2868, 12179, 12405, 15233.

Lithospermum distichum Ortega. 784, 846, 17358, 24376, 26531.

Lithospermum dodrantale (I.M. Johnst.) J. Cohen. 17194, 17378, 23253.

Lithospermum exsertum (D. Don) J.I. Cohen. 1296, 1435, 1541, 9442, 13107, 14809.

Lithospermum hintoniorum B.L. Turner. 21950 (TYPUS), 28641.

Lithospermum indecorum I.M. Johnst. 18128, 22830, 22926.

Lithospermum leonotis (I.M. Johnst.) J.I. Cohen. 17412, 17566, 18523, 18970, 19375, 21026, 22277, 23060.

Lithospermum matamorense DC. 21821, 21868.

Lithospermum mirabile Small. 22729, 27401.

Lithospermum muelleri I.M. Johnst. 18243, 18247.

Lithospermum notatum (I.M. Johnst.) J. Cohen. 18287.

Lithospermum oblongifolium Greenm. 4609, 5372.

Lithospermum parksii I.M. Johnst. 18120, 18143, 18802,

20118, 21839, 21850, 21908, 28203.

Lithospermum strictum Lehm. 1038, 4394, 13023.

Lithospermum trinervium (Lehm.) J. Cohen. 1045, 1246, 1533, 4452, 5064, 8257, 8274, 15274, 15435, 15981, 17417 bis, 17513 bis.

Lithospermum turneri J. Cohen. 14439 (TYPUS).

Lithospermum viride Greene. 17753, 18152, 18718, 18721, 20181, 20288, 22072, 24393, 27178, 28265.

Omphalodes alienoides G.L. Nesom. 29594, 29616.

Omphalodes mexicana S. Watson. 22768.

Omphalodes aliena A. Gray. 16932, 20173, 22773, 28565.

Omphalodes cardiophylla A. Gray ex Hemsl. 19118, 19137, 21027, 24342, 24454, 25906, 28797.

Omphalodes erecta I.M. Johnst. 19196, 21247, 22275, 23142.

Omphalodes mexicana S. Watson. 21036.

Oncaglossum pringlei (Greenm.) Sutorý. 15017, 15930.

\section{Family Brassicaceae}

Asta schaffneri (S. Watson) O.E. Schulz. 18081, 20193, 20490, 27697, 27884, 28146, 29054.

Brassica rapa L. 29821.

Brassica juncea (L.) Cosson. 1624, 3882, 4488, 7277,
15776.

Brassica nigra (L.) K. Koch. 4857, 16781.

Brassica rapa L. 12983, 15535, 18503, 21796, 27034, 27601.

Capsella bursa-pastoris (L.) Medik. 2900, 26647.

Cardamine auriculata S. Watson. 16924, 18118, 18140, 24703.

Cardamine fulcrata Greene. 14468

Cardamine hirsuta L. 3294, 3992, 4077.

Cardamine macrocarpa Brandegee. 19351, 20209, 21813.

Cardamine obliqua Hochst. 489, 6080 (Type of Cardamine obliqua var. stylosa Rollins), 15359, 26646, 26850.

Coelophragmus auriculatus (A. Gray) O.E. Schulz. 18174, 18466, 18586, 20088.

Descurainia impatiens (Cham. \& Schltdl.) O.E. Schulz. 29838.

Descurainia impatiens (Cham. \& Schltdl.) O.E. Schulz. 4611, 4934, 26523, 26690.

Descurainia pinnata (Walter) Britton. 18817, 20117.

Descurainia streptocarpa (Fourn.) O.E. Schulz. 3847.

Descurainia virletii (Fourn.) O.E. Schulz. 18334.

Diplotaxis muralis (L.) DC. 19082, 19505.

Draba helleriana Greene. 17033, 17037, 17050, 17331, 17384, 17973, 19391, 22425.

Draba jorullensis Kunth. 431, 4921.

Eruca sativa Mill. 27612.

Erysimum asperum (Nutt.) DC. 17034, 17386, 17913, 18443, $18458,18662,18845,20147,20259,20303,21782,22419$, 24344, 26790.

Erysimum macradenium A. Gray. 429.

Exhalimolobos polyspermus (E. Fourn.) Al-Shehbaz \& C.D. Bailey. 1314.

Lepidium alyssoides A. Gray. 16713, 17838, 19223, 19424, 24858, 27721, 28808.

Lepidium lasiocarpum Nutt. 19490.

Lepidium schaffneri Thell. 26788.

Lepidium virginicum L. 910, 1109, 3459, 3874, 4236, 6354, 12978, 20115, 29104, 29312.

Mostacillastrum arcuatum (Rollins) Al-Shehbaz. 20722, 21869.

Mostacillastrum purpusii (Brandegee) Al-Shehbaz. 19707.

Mostacillastrum versicolor (Brandegee) Al-Shehbaz. 20310.

Nasturtium officinale R. Br. 656, 3725, 11848, 13662, 13967 , 16770, 17575, 17769, 22165, 22651, 26453.

Nerisyrenia castillonii Rollins. 29564, 29578.

Nerisyrenia camporum (A. Gray) Greene. 18069, 25970, 27428, 28768, 28827, 29125, 29249, 29476, 29599, 29603, 29612.

Nerisyrenia gracilis I.M. Johnst. 16552, 18226, 19337, 19444, 19524, 21947, 24772, 28244, 28328, 28404, 28602, 28615, 28631, 28637, 28684, 28774, 29108.

Nerisyrenia johnstonii J.D. Bacon. 29409, 29426.

Nerisyrenia linearifolia (S. Watson) Greene. 18134, 19653, 19680, 20171, 21804, 23843, 23994, 28443.

Noccaea mexicana (Standl.) Holub. 17021, 17203, 18824, 19392, 20991.

Pennellia longifolia (Benth.) Rollins. 1724, 4920, 6411, 
8389, 17980, 18303, 18867, 18892, 18901, 20447, 22415, 23261.

Phravenia viereckii (O.E. Schulz) Al-Shehbaz \& S.I. Warwick. 20103, 21110, 22008.

Physaria argyraea (A. Gray) O'Kane \& Al-Shehbaz. 18452, 18454, 19607, 19700, 20101, 20123, 20162, 21102, 21776, 27175, 28896, 28973.

Physaria berlandieri (S. Watson) O'Kane \& Al-Shehbaz. 20006, 20342, 21820, 22839, 28108.

Physaria fendleri (A. Gray) O'Kane \& Al-Shehbaz. 17287, 18497, 18833, 19428, 19501, 27196, 28047, 28096, 28245, 28329, 29117.

Physaria inflata (Rollins \& E.A. Shaw) O'Kane \& Al-Shehbaz. 22770.

Physaria mirandiana (Rollins) O'Kane \& Al-Shehbaz. 18501, 18973, 21071, 21904.

Raphanus raphanistrum L. 4696, 6346, 15508, 16731.

Raphanus sativus L. 13872, 17132, 19500.

Rapistrum rugosum (L.) All. 20253.

Romanschulzia meyeri Rollins. 23245.

Romanschulzia orizabae (Schltdl. \& Cham.) O.E. Schulz. 1315.

Rorippa mexicana (Moc. \& Sessé ex DC.) Standl. \& Steyerm. 5611.

Rorippa teres (Michx.) Stuckey. 1120, 4059, 4230.

Scoliaxon mexicanus (S. Watson) Payson. 20164, 21784, 21838

Selenia dissecta Torr. \& A. Gray. 27036, 29093.

Sphaerocardamum divaricatum (Rollins) Rollins. 18499.

Sphaerocardamum stellatum (S. Watson) Rollins. 18138, 20009, 20831, 21455, 21548, 21852, 23589.

Synthlipsis greggii A. Gray. 16772, 18142, 18188, 21840, 28694, 29190.

Thelypodiopsis wootonii (B.L. Rob.) Rollins. 4002.

Thelypodium longipes (Rollins) Rollins. 18542, 20812.

Thelypodium wrightii (A. Gray) Rydb. 18336, 18899, 19188, 19189, 19205, 21003, 21035.

Tomostima cuneifolia (Nutt. ex Torr. \& A. Gray) Al-Shehbaz, M. Koch \& Jordon-Thaden. 18130, 20104, 20116.

\section{Family Bromeliaceae}

Aechmea bracteata (Sw.) Griseb. 10216, 10224, 16048.

Bromelia hemisphaerica Lam. 1221, 6229.

Catopsis compacta Mez. 14062.

Catopsis nutans (Sw.) Griseb. 1842.

Catopsis paniculata E. Morren. 4692 (Type of Catopsis mexicana L.B. Sm.).

Hechtia glomerata Zucc. 20916.

Hechtia hintoniana Burt-Utley, Utley \& García Mend. 6506, 8098.

Hechtia texensis S. Watson. 24185, 25752.

Pitcairnia flexuosa L.B. Sm. 3948 (TYPUS).

Pitcairnia foliacea L.B. Sm. 15941 (TYPUS).

Pitcairnia hintoniana L.B. Sm. 2039bis, 2040 (TYPUS).

Pitcairnia karwinskyana Schult. \& Schult. f. 1219, 3621, 7677, 7719, 10090.

Pitcairnia leprosa L.B. Sm. 10552 (TYPUS), 10653.

Pitcairnia micropoda L.B. Sm. 936 (TYPUS).
Pitcairnia oaxacana L.B. Sm. 11366.

Pitcairnia pteropoda L.B. Sm. 9450, 10121, 10127 (Type of

Pitcairnia militaris L.B. Sm.), 10456.

Pitcairnia ringens Klotzsch. 13890, 13984, 15012.

Pitcairnia roseana L.B. Sm. 11345.

Pitcairnia sordida L.B. Sm. 14248 (TYPUS).

Pitcairnia tillandsioides L.B. Sm. 14398 (TYPUS).

Tillandsia argentea Griseb. 10126.

Tillandsia bartramii Elliott. 21259, 22901, 22988, 25247.

Tillandsia caput-medusae E. Morren. 689, 2856, 3750, 10227.

Tillandsia chaetophylla Mez. 3468, 7449.

Tillandsia dasyliriifolia Baker. 3642.

Tillandsia erubescens Schltdl. 324, 3653, 17341, 17773, 18686, 20944, 21251, 21359, 21880, 25174, 25309, 27491.

Tillandsia fasciculata Sw. 3535, 6375, 7943.

Tillandsia hintoniana L.B. Sm. 7636 (TYPUS), 9736bis.

Tillandsia ionantha Planch. 10110, 10112.

Tillandsia juncea (Ruiz \& Pav.) Poir. 10142.

Tillandsia parryi Baker. 21831, 25169, 25188.

Tillandsia prodigiosa (Lem.) Baker. 7437.

Tillandsia recurvata (L.) L. 10128, 16509, 19596.

Tillandsia schiedeana Steud. 3646, 10133.

Tillandsia thyrsigera E. Morren ex Baker. 3381.

Tillandsia usneoides (L.) L. 23899.

Tillandsia utriculata L. 20934, 22855, 23847.

Viridantha ignesiae (Mez) Espejo. 3762, 7780, 10102.

Viridantha plumosa (Baker) Espejo. 10109, 10129.

\section{Family Burseraceae}

Bursera grandifolia (Schltdl.) Engl. 3910, 9774 (Type of Bursera grandifolia fma. robusta Bullock), 10182.

Bursera aptera Ramírez. 13378.

Bursera ariensis (Kunth) McVaugh \& Rzed. 814, 3733, $3735,3749,6065,6566,6741,6787,7037,7084,7161,7340$, 7629, 7634, 7635, 7645, 7649, 7651, 7652, 7682, 7705, 7798, 7828, 8571, 9049, 9052 (Type of Bursera sessiliflora var. pubivalvis Bullock), 9231, 9273, 9914, 10446, 10553, 12803, 14415.

Bursera aspleniifolia Brandegee. 13910.

Bursera bicolor (Willd. ex Schltdl.) Engl. 3913, 4151, 6128, 6958, 7129, 7730, 9042, 10437, 14418.

Bursera bipinnata (DC.) Engl. 813, 3969, 4183, 4378, 6512, 6789, 7296, 7332, 7569, 7698, 7699, 7729, 7733, 7795, 9199, 11459, 12133, 12557, 13267, 13854, 14045, 16148.

Bursera citronella McVaugh \& Rzed. 12280, 12281, 12282, 12283, 12294, 12295, 12297, 12299 (TYPUS), 12305, 12308, 12311, 12539, 12542, 12543, 12556, 12563, 13900.

Bursera confusa (Rose) Engl. 5952, 6025, 6953, 6992, 7660, 7736, 7772, 7792, 7846, 7847, 7849, 7851, 9062, 9088, 9174, 10641, 12137, 12144, 12306, 12309, 12739, 13290, 13316. Bursera copallifera (Sessé \& Moc. ex DC.) Bullock. 7078, 7331, 7647, 9046, 9048, 9056, 9115, 9116, 9121, 9362, 10017, 12040, 13164, 13252, 13317.

Bursera coyucensis Bullock. 5961, 6272, 6895 (TYPUS), 9047, 9067, 9068, 10057, 10535.

Bursera crenata Paul G. Wilson. 12011, 12025 (TYPUS), 12063, 12071, 12102, 12829, 12831. 
Bursera cuneata (Schltdl.) Engl. 13410.

Bursera denticulata McVaugh \& Rzed. 12021 (TYPUS), 12022, 12081, 14581, 16150.

Bursera discolor Rzed. 6102, 7656, 7796.

Bursera diversifolia Rose. 9262, 10072, 10422, 10428, 12553, 12562, 12575, 12595, 12646, 12686, 12691.

Bursera dubia Bullock. 7877, 9045, 9349 (TYPUS), 10056, 10527, 10643, 12010.

Bursera excelsa (Kunth) Engl. 7723, 10354, 10788, 10851, 10852, 10869, 10937, 12591, 12592, 12600, 14339, 14542, 14565, 16010.

Bursera fagaroides (Kunth) Engl. 4286, 5525, 6126, 6369, 6896, 6899, 7122, 7272, 7394, 7744, 7839, 7842, 7853, 7859, 7866, 7878, 9076, 9120, 9374, 9517, 9518, 10052, 12001, 12002, 12550, 12937, 15221, 29378.

Bursera fragrantissima Bullock. 9255, 9339, 9731 (TYPUS), 10085, 10093, 10190, 10372, 10373, 10375, 10457, 10495, 15207, 15338, 15390.

Bursera glabrifolia (Kunth) Engl. 6790, 7832, 9365, 10654, 12039, 12134, 12565, 13268, 14414, 15220, 15992.

Bursera grandifolia (Schltdl.) Engl. 921, 3804, 3927, 5213, 5705, 6110, 6933, 7123, 7242, 7518, 7626, 7654, 7655, 7657, 7662, 7724, 7737, 7767, 7773, 7802, 7822, 7864, 7870, 7888, $8533,9059,9060,9061,9064,9065,9066,9077,9078,9081$, 9093, 9553, 10187, 10188, 10211, 10226, 10386, 10408, 10420, 10424, 10431, 11451, 12639, 15253, 16279.

Bursera heteresthes Bullock. 7812 (TYPUS), 9044, 9084, 9958, 10055, 10526, 10529, 10642, 10646, 12024, 12587, $14537,14584$.

Bursera hintonii Bullock. 3952, 4298, 6991 (TYPUS), 7774, 7794, 7889, 9108, 9130, 9136, 9246, 9957, 10071, 10091, 10114, 10252, 10440, 14483.

Bursera infernidialis Guevara \& Rzed. 10370.

Bursera kerberi Engl. 7775, 9793.

Bursera laxiflora S. Watson. 10337.

Bursera linanoe (La Llave) Rzed., Calderón \& Medina. 13829.

Bursera longipes (Rose) Standl. 10210, 10212, 10220, 10369.

Bursera multijuga Engl. 3939, 12145.

Bursera occulta McVaugh \& Rzed. 12307, 12310, 12552, 12554, 12555 (TYPUS), 12566, 13813.

Bursera ovalifolia (Schltdl.) Engl. 730, 3787, 4084, 6141, 7140, 7299, 7396, 7801, 7825, 7830, 7831, 10062, 11588, 11731, 12142.

Bursera penicillata (Sessé \& Moc. ex DC.) Engl. 683, 7697, 7728, 7734, 10442, 10443.

Bursera roseana Rzed., Calderón \& Medina. 7328, 11437. Bursera sarcopoda Paul G. Wilson. 12293 (TYPUS), 12608, 12609, 15953.

Bursera schlechtendalii Engl. 25163.

Bursera simaruba (L.) Sarg. 10630, 13805, 13838, 13865, 14033.

Bursera staphyleoides McVaugh \& Rzed. 12073 (TYPUS). Bursera subtrifoliata (Rose) Standl. 12115.

Bursera tecomaca (DC.) Standl. 738, 6192, 7158, 7295, 7300, 7815, 7819, 9086, 9276, 10066, 10414, 10429, 12318.

Bursera trifoliolata Bullock. 780, 3943, 6340 (TYPUS),
7238, 7781, 7841, 7879, 8529, 12074.

Bursera trimera Bullock. 3765, 5943, 5997, 6262 (TYPUS), 6934, 7245, 7590, 7743, 7759, 7765, 7848, 9083, 9106, 9107, 9819, 10061, 10218, 12094, 12095, 12096, 13299, 13323.

Bursera velutina Bullock. 5960, 6124, 6261 (TYPUS), 6894, 7747, 7751, 7760, 9192, 9193 (Type of Bursera velutina var. parvifolia Bullock), 9622, 10016, 10058, 10064, 10530, 10531, 13301.

\section{Family Cactaceae}

Acharagma roseanum (Boed.) E.F. Anderson. 27830.

Ariocarpus fissuratus (Engelm.) K. Schum. 27826.

Ariocarpus fissuratus (Engelm.) K. Schum. 25731 (Type of Ariocarpus fissuratus subsp. hintonii (Stuppy \& N.P. Taylor) Halda), 27141.

Ariocarpus retusus Scheidw. 27147.

Ariocarpus scaphirostris Boed. 27865.

Astrophytum capricorne (A. Dietr.) Britton \& Rose. 27835 , 27836, 28297.

Astrophytum caput-medusae (Velazco \& Nevárez) D.R. Hunt. 28537.

Astrophytum myriostigma Lem. 28551.

Aztekium hintonii Glass \& Fitz Maurice. 21495, 21528, 21529 (TYPUS).

Aztekium ritteri (Boed.) Boed. ex A. Berger. 25732.

Backebergia militaris (Audot) Bravo ex Sánchez-Mej. 5954.

Corynopuntia bulbispina (Engelm.) F.M. Knuth. 28298.

Corynopuntia deinacantha D. Donati. 29070 (TYPUS collected by D. Donati).

Corynopuntia guccinii D. Donati. 29067 (TYPUS collected by D. Donati).

Corynopuntia halophila D. Donati. 29068 (TYPUS collected by D. Donati).

Corynopuntia nigrispina D. Donati. 29071 (TYPUS collected by D. Donati).

Corynopuntia recurvata (Engelm.) Britton \& Rose. 27666 (Type of Coryphantha recurvata subsp. canatlanensis Dicht \& A. Lüthy, collected by R. Richt and A. Lüthy).

Corynopuntia salinensis (Poselger) Dicht \& A. Lüthy. 27113

(TYPUS collected by R. Richt and A. Lüthy).

Coryphantha ramillosa Cutak. 29325.

Coryphantha delicata L. Bremer. 27119, 27121, 27158, 27159, 27162, 27163, 27164, 27172, 27313, 27317, 27330, 27676, 27805.

Coryphantha difficilis (Quehl) A. Berger. 27837, 28293.

Coryphantha glanduligera (Otto \& A. Dietr.) Lem. 27120, 27150, 27165, 27312.

Coryphantha hintoniorum Dicht \& A. Luthy. 27111 (TYPUS), 27166, 27167, 27206 (Type of Coryphantha hintoniorum subsp. geoffreyi Dicht \& A. Lüthy), 27316.

Coryphantha macromeris (Engelm.) Lem. 28352.

Coryphantha nickelsiae (K. Brandegee) Britton \& Rose. 27763, 28273.

Coryphantha ramillosa Cutak. 25792 (Type of Coryphantha ramillosa subsp. santarosa Dicht \& A. Lüthy), 28271.

Cumarinia odorata (Boed.) Buxb. 28557.

Cylindropuntia imbricata (Haw.) F.M. Knuth. 17840, 
18835.

Cylindropuntia leptocaulis (DC.) F.M. Knuth. 17718, 17860, 20626.

Cylindropuntia tunicata (Lehm.) F.M. Knuth. 27136.

Disocactus speciosus (Cav.) Barthlott. 589, 976, 4107.

Echinocactus horizonthalonius Lem. 18178, 25801.

Echinocactus platyacanthus Link \& Otto. 27135.

Echinocereus enneacanthus Engelm. 16609.

Echinocereus dasyacanthus Engelm. 27499.

Echinocereus enneacanthus Engelm. 16611bis, 27137, 27153.

Echinocereus knippelianus Liebner. 18938, 27848.

Echinocereus longisetus (Engelm.) Lem. 27144.

Echinocereus parkeri N.P. Taylor. 22637.

Echinocereus pectinatus (Scheidw.) Engelm. 27840.

Echinocereus pentalophus (DC.) Lem. 18144, 21797, 27731, 27761, 27802.

Echinocereus poselgeri Lem. 27757.

Echinocereus primolanatus A.F. Schwarz ex N.P. Taylor. 27131, 28361.

Echinocereus pulchellus (Mart.) C.F. Först. ex F. Seitz. 27126.

Echinocereus rayonesensis N.P. Taylor. 28119.

Echinocereus reichenbachii (Terscheck ex Walp.) Haage. 27146.

Echinocereus stramineus (Engelm.) Engelm. ex F. Seitz. 27760, 28362.

Echinocereus viridiflorus Engelm. 25770.

Echinomastus mariposensis Hester. 25794, 28300.

Echinomastus warnockii (L. Benson) Glass \& Foster. 27528.

Epiphyllum hookeri Haw. 5402.

Epithelantha micromeris (Engelm.) F.A.C. Weber. 27838, 28272, 28310, 28315, 29326, 29448.

Epithelantha cryptica D. Donati \& Zanov. 29258 (TYPUS collected by D. Donati).

Epithelantha ilariae D. Donati \& Zanov. 29075 (TYPUS collected by D. Donati).

Epithelantha micromeris (Engelm.) F.A.C. Weber ex Britton \& Rose. 29078 (Type of Epithelantha pachyrhiza subsp. pulchra D. Donati \& Zanov., collected by D. Donati), 29211 (Type of Epithelantha pachyrhiza subsp. anjaviana D. Donati \& Zanov., collected by D. Donati), 29228 (Type of Epithelantha unguispina subsp. huastecana D. Donati \& Zanov., collected by D. Donati), 29229 (Type of Epithelantha greggii subsp. potosina D. Donati \& Zanov., collected by D. Donati).

Escobaria emskoetteriana (Quehl) Borg. 27829, 28248, 28274, 28275, 28276, 28316, 28350.

Escobaria missouriensis (Sweet) D.R. Hunt. 27124.

Escobaria tuberculosa (Engelm.) Britton \& Rose. 25779, 25814, 28534.

Ferocactus hamatacanthus (Muehlenpf.) Britton \& Rose. 27139, 27801.

Ferocactus pilosus (Galeotti ex Salm Dyck) Werderm. 18165.

Geohintonia mexicana Glass \& Fitz Maurice. 21519, 21527

(TYPUS).
Glandulicactus uncinatus (Pfeiff \& Otto) Backeb. 27125, 27548.

Grusonia agglomerata (A. Berger) E.F. Anderson. 29080 (EPITYPUS collected by D. Donati, collected by D. Donati).

Grusonia bradtiana (J.M. Coult.) Britton \& Rose. 27132.

Grusonia bulbispina (Engelm.) H. Rob. 29069 (Type of Corynopuntia bulbispina subsp. basileocephala D. Donati).

Lophophora alberto-vojtechii Bohata, Myšák \& Šnicer. 28642 (TYPUS collected by G. Matuszewski).

Mammillaria heyderi Muehlenpf. 28796.

Mammillaria albicoma Boed. 28237.

Mammillaria beneckei C. Ehrenb. 7871, 10330.

Mammillaria formosa Galeotti ex Scheidw. 27151, 27155, 27844.

Mammillaria glassii R.A. Foster. 20973, 21016, 28124, 29310.

Mammillaria heyderi Muehlenpf. 27138, 27168, 27759.

Mammillaria lasiacantha Engelm. 25756, 27118, 28578.

Mammillaria luethyi G.S. Hinton. 25771 (TYPUS).

Mammillaria magnimamma Haw. 18934.

Mammillaria melanocentra Posel. 22646, 28269.

Mammillaria picta Meinsh. 27871.

Mammillaria pilispina J.A. Purpus. 27787, 28123.

Mammillaria plumosa F.A.C. Weber. 28258.

Mammillaria pottsii Scheer ex Salm-Dyck. 27143, 27833, 28351.

Mammillaria prolifera (Mill.) Haw. 25791.

Mammillaria sanchez-mejoradae R. González G. 27140.

Mammillaria sempervivi DC. 27803.

Mammillaria sphaerica A. Dietr. 27762.

Mammillaria uncinata Zucc. ex Pfeiff. 18808.

Mammillaria weingartiana Boed. 27123.

Mammillaria winterae Boed. 27152, 27843, 27849.

Mammilloydia candida (Scheidw.) Buxb. 28238, 28561.

Neolloydia conoidea (DC.) Britton \& Rose. 16611, 27133, 29355, 29474.

Neolloydia inexpectata D. Donati. 29254 (TYPUS collected by D. Donati).

Opuntia streptacantha Lem. 29866.

Opuntia tomentosa Salm-Dyck. 29867.

Opuntia auberi Pfeiff. 3203, 7170.

Opuntia dejecta Salm-Dyck. 25256.

Opuntia phaeacantha Engelm. 17839, 17841, 18836, 18837.

Opuntia stenopetala Engelm. 16641, 18726.

Pachycereus pecten-aboriginum (Engelm. ex S. Watson) Britton \& Rose. 6209.

Pelecyphora aselliformis Ehrenb. 27142, 27881.

Pelecyphora strobiliformis (Werderm.) Fric \& Schelle. 27827.

Peniocereus greggii (Engelm.) Britton \& Rose. 28292.

Peniocereus serpentinus (Lag. \& Rodr.) N.P. Taylor. 6196. Pereskiopsis rotundifolia (DC.) Britton \& Rose. 6273.

Pilosocereus alensis (F.A.C. Weber) Byles \& G.D. Rowley. 5948, 7761.

Sclerocactus brevihamatus (Engelm.) D.R. Hunt. 29472 (Type of Ancistrocactus pinkavanus L. García-Mor., M. 
González-Botello \& R. González G., collected by Lex García and Miguel González).

Sclerocactus scheeri (Salm-Dyck) N.P. Taylor. 28270, 28294.

Selenicereus boeckmannii (Otto) Britton \& Rose. 13796.

Stenocereus pruinosus (Otto ex Pfeiff.) Buxb. 28291, 28550.

Thelocactus bicolor (Galeotti) Britton \& Rose. 27128, 27331.

Thelocactus conothelos (Regel \& Klein) Knuth. 28239.

Thelocactus hexaedrophorus (Lem.) Britton \& Rose. 27343.

Thelocactus rinconensis (Poselg.) Britton \& Rose. 21775, 25778 (Type of Thelocactus rinconensis subsp. hintonii J. Lüthy), 27129.

Thelocactus setispinus (Engelm.) E.F. Anderson. 27758.

Thelocactus tulensis (Poselg.) Britton \& Rose. 28560.

Turbinicarpus beguinii (N.P. Taylor) Mosco \& Zanovello. 18379, 18818, 27134, 27194, 27207, 27208, 27327, 27675, 27828, 27845.

Turbinicarpus beguinii (N.P. Taylor) Mosco \& Zanovello. 24010, 27023 (Type of Turbinicarpus beguinii subsp. hintoniorum A. Hofer).

Turbinicarpus booleanus G.S. Hinton. 21805 (TYPUS).

Turbinicarpus graminispinus Matusz., V. Mysak \& Z. Jiruse. 29084 (TYPUS collected by V. Mysak).

Turbinicarpus hoferi Luthy \& A.B. Lau. 27110, 27872.

Turbinicarpus mandragora (Fric ex A. Berger) A.D. Zimmerman. 25799.

Turbinicarpus pseudopectinatus (Backeb.) Glass \& R. Foster. 27173, 27342.

Turbinicarpus saueri (Boed.) Vác. John \& Ríha. 28256 (Type of Turbinicarpus saueri subsp. septentrionalis Matusz. \& Snicer, collected by G. Matuszewski).

Turbinicarpus schmiedickeanus (Boed.) Buxb. \& Backeb. 27329, 27869, 27870.

Turbinicarpus subterraneus (Backeb.) A.D. Zimmerman. 27210.

Turbinicarpus valdezianus (Moell.) Glass \& R. Foster. 27315, 28240.

Turbinicarpus viereckii (Werderm.) Vác. John \& Riha. 29081 (Type of Turbinicarpus viereckii subsp. reconditus D. Labhart, collected by D. Labhart).

Turbinicarpus zaragozae (Glass \& R.A. Foster) Glass \& A. Hofer ex Glass. 27226.

\section{Family Calceolariaceae}

Calceolaria mexicana Benth. 817, 14797, 17085, 22745, 24092, 26046, 26310, 26313, 26594, 26707.

Calceolaria trilobata Hemsl. 26106.

\section{Family Campanulaceae}

Calcaratolobelia cordifolia (Hook. \& Arn.) Wilbur. 2560, 2995, 5295, 6920, 12859, 12866, 16298.

Calcaratolobelia margarita (E. Wimm.) Wilbur. 18101, 18746, 21580, 21721, 21867, 21962, 22316.

Calcaratolobelia pringlei (B.L. Rob.) Wilbur. 19307, 19330, 19691, 20388, 20597, 23116, 23333, 23988, 25525, 25717,
$27373,28717$.

Calcaratolobelia tenella (Turcz.) Wilbur. 7107.

Campanula rotundifolia L. 17105, 17306, 17923, 18583, 20449, 21282, 22288, 22580, 23522.

Centropogon grandidentatus (Schltdl.) Zahlbr. 11227, 14220, 26289, 26600.

Diastatea micrantha (Kunth) McVaugh. 29802, 29857.

Diastatea expansa McVaugh. 2644 (TYPUS), 7149, 8625, 13393.

Diastatea micrantha (Kunth) McVaugh. 2852, 5276, 8543, 8655, 26399.

Diastatea tenera (A. Gray) McVaugh. 183, 2122, 2211, 2507, 8488, 8661, 14860.

Diastatea virgata Scheidw. 8538.

Heterotoma lobelioides Zucc. 13780, 13801.

Lobelia fenestralis Cav. 29711.

Lobelia gruina Cav. 18065, 29740.

Lobelia longicaulis Brandegee. 7195.

Lobelia sartorii Vatke. 18109.

Lobelia berlandieri A. DC. 17422, 20170.

Lobelia cardinalis L. 1107, 1774, 4616, 4679, 9680, 13208, 15718, 19961, 20067, 21556, 23291.

Lobelia divaricata Hook. \& Arn. 17315.

Lobelia ehrenbergii Vatke. 20734.

Lobelia fenestralis Cav. 833, 1092, 1148, 1658, 4457, 15454.

Lobelia gruina Cav. 2114, 2451, 8323.

Lobelia hintoniorum B.L. Turner. 26104, 26256, 26265.

Lobelia irasuensis Planch. \& Oerst. 1714, 4618, 8249.

Lobelia laxiflora Kunth. 210, 1403, 2714, 8910, 11311, 12919, 15025, 15523, 17484bis, 26118, 26270, 26295.

Lobelia longicaulis Brandegee. 2450, 5086, 17170.

Lobelia macdonaldii B.L. Turner. 26146, 26151, 26786.

Lobelia pulchella Vatke. 14881.

Lobelia sublibera S. Watson. 17534, 21371, 21703, 22300, 22626, 22686, 22887, 22906, 23927, 24129, 24234, 25324.

Lobelia trivialis E. Wimm. 18277, 19233, 22161, 23397. 24482, 28037.

\section{Family Cannabaceae}

Aphananthe monoica (Hemsl.) J.F. Leroy. 13946.

Cannabis sativa L. 7694.

Celtis caudata Planch. 4347, 6383, 7646, 9592.

Celtis ehrenbergiana (Klotzsch) Liebm. 7630, 16846, 17775, 21957, 22172, 24068.

Celtis iguanaea (Jacq.) Sarg. 779, 3924, 4136, 6294, 7002, 7527, 7869 .

Celtis laevigata Willd. 12328, 16828, 19358, 24364, 24969.

Lozanella enantiophylla (Donn. Sm.) Killip \& C.V. Morton. 14974.

Trema micrantha (L.) Blume. 439, 1056, 3374, 3532, 7833, 8723, 9274, 10484, 10948, 14028, 16260.

Family Cannaceae

Canna indica L. 1670, 11386, 16165.

Family Capparaceae 
Crateva palmeri Rose. 5467, 5731, 5886, 15863.

Crateva tapia L. 10320, 14254.

Cynophalla flexuosa (L.) J. Presl. 15914.

Cynophalla verrucosa (Jacq.) J. Presl. 10336, 10341, 13771, 15820.

Forchhammeria hintonii Paul G. Wilson. 5746, 7401, 7571 (TYPUS).

Forchhammeria pallida Liebm. 7273, 7275.

Forchhammeria trifoliata Radlk. 14002.

Quadrella angustifolia (Kunth) H.H. Iltis \& X. Cornejo. 12049, 12113, 12828, 15868.

Quadrella asperifolia (C. Presl) H.H. Iltis \& X. Cornejo. 11800, 14131.

Quadrella indica (L.) H.H. Iltis \& X. Cornejo. 13755, 13821, 15874.

\section{Family Caprifoliaceae}

Abelia coriacea Hemsl. 19266.

Lonicera albiflora Torr. \& A. Gray. 16771, 18358, 18406.

Lonicera pilosa (Kunth) Willd. ex Kunth. 650, 3867, 9010, 17127, 17249, 17370, 18724, 20270, 23262, 23320, 25425.

Symphoricarpos microphyllus Kunth. 607, 1031, 13120, 13392, 17115, 17387, 18293, 18343, 20992, 23974.

Valeriana sorbifolia Kunth. 29719.

Valeriana urticifolia Kunth. 29707.

Valeriana albonervata B.L. Rob. 22947.

Valeriana apiifolia A. Gray. 14733.

Valeriana clematitis Kunth. 487, 2732, 19119, 19382, 20152.

Valeriana densiflora Benth. 894, 981, 1368, 4164, 4395, 17552.

Valeriana naidae Barrie. 8981.

Valeriana oaxacana Barrie. 1097.

Valeriana pratensis (Benth.) Steud. 10501.

Valeriana pulchella M. Martens \& Galeotti. 26053, 26182, 26559, 26645, 26650, 26693, 26765, 26811.

Valeriana scandens L. 3240, 14719, 17710, 22429, 23976.

Valeriana sorbifolia Kunth. 1115, 1513, 1793, 2180, 2416, 2630, 2760, 2889, 4625, 4636, 7217, 7951, 7989, 8955, 9938, 10474, 10702, 11380, 11998, 12518, 12808, 13481, 14386, $15004,15031,17166,17181,17237,18923,19022,22387$, 23308, 23434, 23462, 23809.

Valeriana tanacetifolia F.G. Mey. 1663 (TYPUS), 7960.

Valeriana urticifolia Kunth. 1677, 2849, 4646, 7981, 9412, 9468, 9846, 10701, 11167, 11335, 12189, 14664, 15040, 15269, 15460, 15550, 17432bis.

Valeriana vaginata Kunth. 608.

\section{Family Caricaceae}

Carica papaya L. 3218, 6179.

Jacaratia mexicana A. DC. 3012, 3213, 3359.

\section{Family Caryophyllaceae}

Arenaria bourgaei Hemsl. 873, 4097, 8342, 17333.

Arenaria gypsostrata B.L. Turner. 20383, 23706 (TYPUS), 23998, 28707.

Arenaria hintoniorum B.L. Turner. 20957 (TYPUS), 28243, 28262.
Arenaria lanuginosa (Michx.) Rohrb. 875, 3842, 3865, 4462, 4586, 9290, 11230, 11925, 11935, 15272, 18814, 21871, 22007, 22252, 22389, 22410, 22622, 22821, 23279, 23395, 26215, 26239, 26545, 26802, 27530.

Arenaria lycopodioides Willd. ex Schltdl. 554, 850, 18532 , 18741, 19713, 20861, 22020, 22822, 24923, 25440, 26537. Arenaria oresbia Greenm. 17009, 17101, 17291, 17910. Arenaria paludicola B.L. Rob. 3991.

Arenaria reptans Hemsl. 863, 3853.

Cerastium brachypodum (Engelm. ex A. Gray) B.L. Rob. ex Britton. 18827, 18853, 21134.

Cerastium hintoniorum B.L. Turner. 26114 (TYPUS).

Cerastium nutans Raf. 868, 4199, 8346, 11231, 15095, 26026, 26183.

Cerastium viscosum L. 2888.

Corrigiola andina Triana \& Planch. 3592.

Drymaria tenuis S. Watson. 29710.

Drymaria anomala S. Watson. 23387.

Drymaria barkleyi J.A. Duke \& Steyerm. 28618.

Drymaria cordata (L.) Willd. ex Roem. \& Schult. 1549, 2596, 5216, 7442, 11538, 12167, 12233, 12489, 15050, 15505.

Drymaria elata I.M. Johnst. 28835.

Drymaria excisa Standl. 508, 2811, 4919, 5074, 5427 (Type of Drymaria grandis Bullock), 5429, 9007.

Drymaria gracilis Schltdl. \& Cham. 5152, 6578, 15263, 23466, 25676, 28953.

Drymaria jenniferae Villarreal \& A. Estrada. 28498 (TYPUS).

Drymaria laxiflora Benth. 21135.

Drymaria longepedunculata S. Watson. 1957.

Drymaria lyropetala I.M. Johnst. 25716, 25989, 27877 , 28469, 28606, 28630.

Drymaria pachyphylla Wooton \& Standl. 28299.

Drymaria polycarpoides A. Gray. 28536.

Drymaria subumbellata I.M. Johnst. 28357, 29365, 29406, 29430, 29588, 29604.

Drymaria villosa Schltdl. \& Cham. 1384, 3304, 7613, 11284, 18129.

Minuartia moehringioides (Moc. \& Sessé ex Ser.) Mattf. 2012, 2443, 4448, 4542, 8640, 17222.

Paronychia hintoniorum B.L. Turner. 25368 (TYPUS), 27031.

Polycarpon tetraphyllum (L.) L. 1892, 4615.

Saponaria officinalis L. 24341.

Silene laciniata Cav. 17196, 17696, 17981, 18905, 19070, 19164, 19197, 20429, 20741, 21288, 22396, 24713.

Spergula arvensis L. 1903, 2458, 4536, 8407.

Stellaria cuspidata Willd. ex Schltdl. 4192, 5083, 7348, 18122, 20217, 20241, 22386, 22669, 22919, 23801, 24015, 24017, 24112, 25019, 25081, 28157.

Stellaria hintoniorum B.L. Turner. 17040, 17108 (TYPUS), 17389, 18869, 20307.

Stellaria miahuatlana B.L. Turner. 26426 (TYPUS).

Stellaria nemorum L. 2694, 3068, 4009, 8635, 8983, 11132 , $11525,11849$.

Stellaria ovata Willd. ex Schltdl. 1711, 3999, 4076, 7461, 12372, 12380. 


\section{Family Celastraceae}

Celastrus pringlei Rose. 290, 3506, 3717, 7230, 7380, 7678, 9020 .

Euonymus corymbosus Sprague \& Bullock. 244, 315bis, 325 (TYPUS).

Hippocratea volubilis L. 3657, 11794.

Maytenus phyllanthoides Benth. 17845, 18790, 21956, 27052, 28743.

Maytenus repanda Turcz. 3574.

Mortonia greggii A. Gray. 16803, 17817.

Mortonia latisepala I.M. Johnst. 16545, 25755.

Mortonia palmeri Hemsl. 18399, 18403, 18415, 18695, 19754, 21899, 21964.

Mortonia scabrella A. Gray. 29290.

Orthosphenia mexicana Standl. 18067, 18076, 27130.

Paxistima myrsinites (Pursh) Raf. 18158, 18811, 19452.

Pristimera celastroides (Kunth) A.C. Sm. 3633, 4152, 5687, 5743, 7251, 9973, 9992.

Schaefferia cuneifolia A. Gray. 27506.

Semialarium mexicanum (Miers) Mennega. 3622, 7595.

Wimmeria lanceolata Rose. 6963.

Wimmeria persicifolia Radlk. 3151, 11294, 13218, 13300.

Zinowiewia concinna Lundell. 2332, 3253, 6834, 13446, 15653, 17505bis.

\section{Family Cistaceae}

Helianthemum glomeratum (Lag.) Lag. ex DC. 29818.

Helianthemum concolor (L. Riley) J.G. Ortega. 3190, 12960.

Helianthemum glomeratum (Lag.) Lag. ex DC. 368, 2867, 8401, 8916, 15733, 21634.

\section{Family Cleomaceae}

Andinocleome magnifica (Briq.) Iltis \& Cochrane. 9008.

Andinocleome pilosa (Benth.) Iltis \& Cochrane. 10972bis, 16019.

Cleome fosteriana H.H. Iltis. 9408, 10537 (Type of Physostemon aureum R.C. Foster), 13297.

Cleoserrata serrata (Jacq.) H.H. Iltis. 12469.

Cleoserrata speciosa (Raf.) H.H. Iltis. 1243, 1660, 4725, 4846, 7007, 7103, 9239, 9292, 9595, 11472, 13142.

Gynandropsis gynandra (L.) Briq. 4567, 5499, 6881, 15849.

Morisonia americana L. 7597.

Physostemon guianense (Aubl.) Malme. 4222, 4441, 6282, 6289, 6312, 9075, 10533.

Physostemon hemsleyanum (Bullock) R.C. Foster. 9110.

Physostemon melanospermum (S. Watson) Pax \& K. Hoffm. 10598.

Polanisia uniglandulosa (Cav.) DC. 16877, 17622, 21445, 22865, 23122, 23484, 24429, 24975.

Tarenaya spinosa (Jacq.) Raf. 14284.

\section{Family Clethraceae}

Clethra hartwegii Britton. 13536.

Clethra lanata M. Martens \& Galeotti. 7421.

Clethra mexicana DC. 186, 645, 2650, 5912, 5917, 7668, $10080,10302,10401,13494,13746,14202,14318$, 17529bis.

Clethra occidentalis (L.) Kuntze. 12789, 14079, 14145.

Clethra pringlei S. Watson. 23284, 23821, 24520.

\section{Family Clusiaceae}

Clusia salvinii Donn. Sm. 1703, 2087, 4669, 4670, 7439, 10776, 13864, 15492.

Garcinia intermedia (Pittier) Hammel. 16144.

Family Combretaceae

Combretum decandrum Jacq. 3018, 3172, 3390, 5475, 11728.

Combretum fruticosum (Loefl.) Stuntz. 728, 922, 2885, 3364, 5530, 5632, 6913, 9977, 10834, 11690.

Laguncularia racemosa (L.) C.F. Gaertn. 26496.

\section{Family Commelinaceae}

Callisia filiformis (M. Martens \& Galeotti) D.R. Hunt. 4748, 6476.

Callisia fragrans (Lindl.) Woodson. 11587.

Callisia hintoniorum B.L. Turner. 22568, 23112 (TYPUS), 23699, 23748, 23848, 25725.

Callisia navicularis (Ortgies) D.R. Hunt. 18284, 18457, 18492, 19646, 20409, 20718, 21124, 24494, 25328, 27825.

Commelina coelestis Willd. 18003, 18024, 29714.

Commelina coelestis Willd. 15391.

Commelina dianthifolia Delile. 1738, 9368, 9487, 11454, 13040, 13127, 17212, 17232, 17414bis, 22888, 23315, 24714, 27216, 27660, 27812

Commelina diffusa Burm. f. 6980, 10711, 11854, 20551, 21020, 22261, 23391, 23485, 25396.

Commelina erecta L. 10877, 13868, 14590, 16575, 17959 , 20776, 21104, 22377, 23172, 23361, 23720, 23747, 24237 , 24330, 24493, 24743, 24843, 25523, 25859, 28544, 28638, 28713.

Commelina leiocarpa Benth. 8765, 9486, 9960, 13869.

Commelina scabra Benth. 1087.

Commelina tuberosa L. 1121, 1544, 2397, 7011, 9562, 9672, 15642, 21430, 22217, 22226, 22883, 23174, 23206, 23225, 24600, 26039.

Gibasis gypsophila B.L. Turner. 22082 (TYPUS), 22376, 23406, 23482, 23758.

Gibasis hintoniorum B.L. Turner. 23013 (TYPUS), 27063. Gibasis karwinskyana (Schult. f.) Rohweder. 17557, 18991, 19612, 19758, 20811, 21023, 21286, 21453, 22140, 22271, 22886, 22915, 23076, 23386, 23714, 24401, 24677, 25142, 25841, 27214, 27403, 27659, 27806, 28546.

Gibasis linearis (Benth.) Rohweder. 1021, 1126, 4216, 4384, 13875, 13998, 23362.

Gibasis pellucida (M. Martens \& Galeotti) D.R. Hunt. 10710, 10764, 24100, 24631, 25018, 25528.

Gibasis pulchella (Kunth) Raf. 1726, 1745, 13259.

Gibasis venustula (Kunth) D.R. Hunt. 21055, 21056, 25963, 28777.

Matudanthus nanus (M. Martens \& Galeotti) D.R. Hunt. 26566, 26634.

Murdannia nudiflora (L.) Brenan. 665.

Thyrsanthemum goldianum D.R. Hunt. 13082 (TYPUS). 
Thyrsanthemum macrophyllum (Greenm.) Rohweder. 4368, 4436, 10505, 13139.

Tinantia erecta (Jacq.) Schltdl. 818, 4883, 4978, 8444, 9753, 9771, 10686, 10708, 11685, 12175, 13181, 14622, 15057, 15343, 15507, 15974, 26011, 26712.

Tinantia leiocalyx C.B. Clarke ex Donn. Sm. 1647, 1843, 2157, 8370, 9167, 12227, 15142, 15985.

Tinantia pringlei (S. Watson) Rohweder. 17592, 17646, 21349, 22597, 24109, 24231, 28569.

Tradescantia brevifolia (Torr.) Rose. 27775, 27815.

Tradescantia commelinoides Schult. \& Schult. f. 6353, 8105, 10504, 13012, 13944, 15093, 15353, 17171, 26055, 26359, 26434, 26626, 26665.

Tradescantia crassifolia Cav. 1095, 10879, 11879, 13977, 17416, 27238.

Tradescantia guatemalensis C.B. Clarke. 26312.

Tradescantia gypsophila B.L. Turner. 29343.

Tradescantia hirta D.R. Hunt. 18276, 18494, 19647, 20297, 20410, 21478, 21560, 23385, 23513, 23605, 23627, 23694, 24495, 25395, 28706.

Tradescantia nuevoleonensis Matuda. 18493, 18972, 21105, 21113, 27623, 27678, 28479.

Tradescantia orchidophylla Rose \& Hemsl. 1124, 8037, 12008.

Tradescantia pallida (Rose) D.R. Hunt. 24274, 24630.

Tradescantia sillamontana Matuda. 20785, 25665, 25671, 27407, 28254, 28409, 28562, 28639, 28712.

Tripogandra amplexans Handlos. 16050.

Tripogandra amplexicaulis (Klotzsch ex C.B. Clarke) Woodson. 8762, 9260, 9504, 12219, 13198.

Tripogandra angustifolia (B.L. Rob.) Woodson. 1494, 27213.

Tripogandra disgrega (Kunth) Woodson. 468, 2089, 2231, 10712, 12242.

Tripogandra palmeri (Rose) Woodson. 12051.

Tripogandra purpurascens (S. Schauer) Handlos. 4821.

Weldenia candida Schult. f. 790, 1110, 9963, 26584, 26638 .

\section{Family Connaraceae}

Rourea glabra Kunth. 11353, 14594, 15902.

\section{Family Convolvulaceae}

Bonamia elliptica (L.B. Sm. \& B.G. Schub.) Myint \& D.B. Ward. 2176 (TYPUS), 8487.

Bonamia repens (I.M. Johnst.) D.F. Austin \& Staples. 25760.

Calycobolus nutans (Choisy) D.F. Austin. 5531, 7268, 13527, 16223.

Calycobolus velutinus (M. Martens \& Galeotti) House. 3116 , 7096.

Convolvulus arvensis L. 17138, 19421.

Convolvulus equitans Benth. 16787, 20529, 21926, 22010, 24214, 24427, 27181.

Convolvulus nodiflorus Desr. 11507, 13335, 16125.

Cressa truxillensis Kunth. 28744.

Cuscuta americana L. 5542, 6911.

Cuscuta applanata Engelm. 23503.
Cuscuta boldinghii Urb. 10883.

Cuscuta corymbosa Ruiz \& Pav. 3425, 8984.

Cuscuta gracillima Engelm. 2171, 2497, 5131.

Cuscuta jalapensis Schltdl. 21213.

Cuscuta macvaughii Yunck. 12098.

Cuscuta mitriformis Engelm. ex Hemsl. 17631, 20029.

Cuscuta odontolepis Engelm. 2604.

Cuscuta ortegana Yunck. 16294.

Cuscuta pentagona Engelm. 20755.

Cuscuta tinctoria Mart. ex Engelm. 2814.

Dichondra argentea Humb. \& Bonpl. ex Willd. 17611, 18981, 19696, 23956, 25898.

Evolvulus alsinoides (L.) L. 1007, 1071, 1227, 1981, 2986, 4501, 5090, 5091, 5188, 6629, 8391, 9080, 9520, 9582, $10390,10820,11423,11446,11946,15246,15251,16259$, 17528, 17672, 21116, 21152, 23366, 24268, 25518, 28920.

Evolvulus cardiophyllus Schltdl. 10857.

Evolvulus filipes Mart. 4757.

Evolvulus nummularius (L.) L. 4949, 15314, 16045, 16107. Evolvulus rotundifolius (S. Watson) Hall. f. 20834, 23404.

Evolvulus sericeus Sw. 4480, 11909, 22986, $24559,27639$. Ipomoea madrensis S. Watson. 29741.

Ipomoea orizabensis (Pelletan) Ledeb. ex Steud. 18023. Ipomoea purpurea (L.) Roth. 18014.

Ipomoea alba L. 5011, 7169, 7587, 8414, 11195, 12698, 12969.

Ipomoea ampullacea Fernald. 2928, 4786, 8258, 8590, 8610, 11730.

Ipomoea aristolochiifolia (Kunth) G. Don. 5173, 5173bis, 6984 (Type of Ipomoea cordata L.B. Sm. \& B.G. Schub.), 7329, 8535, 8555, 8588, 9675, 9693, 9785, 12258, 12259, $16678,21674,24004$.

Ipomoea batatas (L.) Lam. 5511.

Ipomoea batatoides Choisy. 9815.

Ipomoea bracteata Cav. 1749, 3019, 5560, 7526 (Type of Ipomoea bracteata var. viridibracta J.A. McDonald), 7529, 9974.

Ipomoea capillacea (Kunth) G. Don. 1275, 1407, 4862, 6523, 8009, 8227, 8456, 9211, 13184.

Ipomoea carnea Jacq. 12514, 17952.

Ipomoea clavata (G. Don) J.F. Macbr. 9676, 10916, 11608, 16188.

Ipomoea cordatotriloba Dennst. 20526.

Ipomoea costellata Torr. 15439.

Ipomoea cristulata Hallier f. 17674, 26458.

Ipomoea decemcornuta O'Donell. 4991 (TYPUS), 12271.

Ipomoea dimorphophylla Greenm. 1382, 8262, 8554, 10519 , 11480, 12464, 13325, 15188, 15254.

Ipomoea dumosa (Benth.) L.O. Williams. 12332.

Ipomoea elongata Choisy. 9646, 9703, 11542.

Ipomoea emetica Choisy. 6525, 8442, 8474 (Type of Ipomoea hintonii L.O. Williams).

Ipomoea funis Schltdl. \& Cham. 9752, 9866, 14969, 15394. Ipomoea hastigera Kunth. 5750, 11196, 11723.

Ipomoea hederifolia L. 1759, 2550, 5132, 5163, 6646, 6875, 7114, 8508, 9528, 10895, 11061, 11166, 11471, 12333, 12454, 12704, 12729, 13250, 14975.

Ipomoea hirta M. Martens \& Galeotti. 8557. 
Ipomoea ignava House. 8563.

Ipomoea igualensis Weath. 2164, 8480, 9588, 12313, 15235.

Ipomoea indica (Burm.) Merr. 2004, 5512, 6641, 10899, $11432,12466$.

Ipomoea invicta House. 11766.

Ipomoea jalapa (L.) Pursh. 17942, 20363, 21456, 22804, 24198, 24605, 24785.

Ipomoea lindenii M. Martens \& Galeotti. 4331, 8592, 9589, 9769, 11561, 16038.

Ipomoea lindheimeri A. Gray. 16838.

Ipomoea lobata (Cerv.) Thell. 2248, 5070, 5631, 7092, 9638, 13258.

Ipomoea madrensis S. Watson. 1234.

Ipomoea meyeri (Spreng.) G. Don. 8576, 9512, 9799, 9808, 9898, 11499.

Ipomoea microsepala Benth. 11002, 11658, 12614, 14142, 15797, 16189.

Ipomoea minutiflora (M. Martens \& Galeotti) House. 4997, 5002, 10864, 13307, 15250.

Ipomoea miquihuanensis J.A. McDonald. 19261.

Ipomoea muricata (L.) Jacq. 6932.

Ipomoea murucoides Roem. \& Schult. 2786, 3360, 5887, 8730, 8755, 12692, 13562.

Ipomoea neei (Spreng.) O'Donell. 3199, 3320, 13656, 15773, 15846.

Ipomoea nil (L.) Roth. 5500, 8208, 8461, 8521, 9511, 9636, 9674.

Ipomoea orizabensis (Pelletan) Ledeb. ex Steud. 8007, 8417, 9566, 9645, 9728, 11922, 12339, 12801, 15226, 15283, 15461, 17457bis, 17645, 17657, 17667, 22294, 22457, 22499, 23276, 23668, 24876, 28506.

Ipomoea parasitica (Kunth) G. Don. 5510, 8591, 9634, 9721, 10888, 10898, 11247, 11716, 12447, 12544, 15302.

Ipomoea pes-caprae (L.) R. Br. 10867, 26468.

Ipomoea populina House. 11528.

Ipomoea praecana House. 8739.

Ipomoea punticulata Benth. 9907, 11612, 12594, 12615.

Ipomoea purga (Wender.) Hayne. 479, 2220, 8594, 9479, 9637, 11207, 12254, 13427.

Ipomoea purpurea (L.) Roth. 593, 1820, 4606, 5414, 6511, $6908,8131,8196,8413,8415,8454,9639,9664,9694$, 12662, 12999, 20474, 20752, 23483, 25303, 27662, 27663. Ipomoea quamoclit L. 5022, 10848, 10932, 11433.

Ipomoea robinsonii House. 6543.

Ipomoea rupicola House. 24248, 25751.

Ipomoea santillanii O'Donell. 2296, 8612, 12680, 16051. Ipomoea seducta House. 10813, 10892, 11770, 12700.

Ipomoea sescossiana Baill. 27080.

Ipomoea setosa Ker Gawl. 9896, 12613, 26487.

Ipomoea simulans T. Hanb. 8348.

Ipomoea suaveolens (M. Martens \& Galeotti) Hemsl. 8528, 13324.

Ipomoea suffulta (Kunth) G. Don. 1606, 1631, 8199, 8420, 8450, 9271, 9398, 9415, 9557, 11363, 13188.

Ipomoea temascaltepecensis P. Wilkin. 5316.

Ipomoea ternifolia Cav. 1822, 5176, 5459, 6471, 8023, 8499, $9272,13216,13327,15159$.
Ipomoea tiliacea (Willd.) Choisy. 3434, 8192, 8416, 8479, 8501, 8504, 9401, 9510, 9699, 9784, 10915, 11465, 11519, 12513, 13130, 15309.

Ipomoea trifida (Kunth) G. Don. 14665, 17612, 21558.

Ipomoea violacea L. 9722, 9723, 9842, 12496, 12497, 12501, 12515, 12838.

Ipomoea wolcottiana Rose. 357, 447, 5305, 7404, 8754, 13406, 16241.

Jacquemontia nelsonii House. 9897, 10835, 11575, 12312, 16009.

Jacquemontia pentanthos (Jacq.) G. Don. 2177, 2474, 4704, 5124, 6638, 6874, 8507, 8510, 8541, 8553, 9814, 11615, 13217, 15318, 25148.

Jacquemontia pycnocephala Benth. 10872, 12604.

Jacquemontia tamnifolia (L.) Griseb. 5207, 6715, 9516, 11435.

Merremia aegyptia (L.) Urb. 4135, 5158, 5621, 8524, 9804, 11650, 12617, 26478.

Merremia cissoides (Lam.) Hallier f. 11778.

Merremia dissecta (Jacq.) Hallier f. 24279, 25479.

Merremia platyphylla (Fernald) O'Donell. 11748, 16103.

Merremia quinquefolia (L.) Hallier f. 2178, 3439, 5175, 5466, 5477, 10796, 10873, 13586, 15320, 15330.

Merremia umbellata (L.) Hallier f. 3126, 5526, 5668, 7167, 10027, 11761, 12926, 13605, 16218.

Operculina pinnatifida (Kunth) O'Donell. 3743, 4772, 5503, 6493, 7748, 8157, 10952, 13819.

Operculina pteripes (G. Don) O'Donell. 1973, 2170, 4817, 6499, 9421, 10629, 13296, 14495.

Turbina corymbosa (L.) Raf. 2276, 2651, 5212, 8238, 9783, 11646, 12335, 12597, 13215.

\section{Family Cordiaceae}

Cordia alliodora (Ruiz \& Pav.) Oken. 11734.

Cordia boissieri A. DC. 16676, 17737, 19271, 19942.

Cordia coyucana I.M. Johnst. 8156 (TYPUS).

Cordia dentata Poir. 3755, 5616, 9513, 10953, 15801.

Cordia elaeagnoides DC. 2611, 5378, 6012, 12111, 13293, 16190.

Cordia gracilipes I.M. Johnst. 14826 (TYPUS).

Cordia morelosana Standl. 3122, 5702, 9981, 10034, 10046, 12951, 13611.

Cordia parvifolia A. DC. 24731, 28434, 28815.

Cordia prunifolia I.M. Johnst. 11048.

Cordia salvadorensis Standl. 4141, 4266, 7740 (Type of Cordia hintonii I.M. Johnst.), 7918, 8048, 8169, 13914, 15066 (Type of Cordia subvelutina I.M. Johnst.).

Cordia seleriana Fernald. 12622, 13768, 13769.

Cordia tinifolia Willd. ex Roem. \& Schult. 3176.

Cordia truncatifolia Bartlett. 10342.

Varronia bullata L. 1164, 13209.

Varronia curassavica Jacq. 6120, 6485, 7256, 9356, 10050, 12042, 12109.

Varronia inermis (Mill.) Borh. 4213, 4337, 6510, 13248.

Varronia macrocephala Desv. 12072.

Varronia oaxacana (DC.) Friesen. 10256, 10542.

Varronia podocephala (Torr.) Borh. 21496.

Varronia spinescens (L.) Borh. 13609, 13669, 14368. 


\section{Family Coriariaceae}

Coriaria ruscifolia L. 14201, 15398, 15448, 15546.

\section{Family Cornaceae}

Cornus disciflora Moc. \& Sessé ex DC. 421, 906, 3292, 3554, 5758, 8837, 13558, 17508bis, 22528, 22617, 22688, 22742, 25184.

Cornus excelsa Kunth. 466, 602, 905, 6799, 10168, 11859. Cornus florida L. 21321, 21640, 22649, 22744, 24892, 25079, 25284.

Cornus stolonifera Michx. 17054, 18427, 19455, 22928, 22950, 24306, 24699, 25301.

\section{Family Costaceae}

Costus pictus D. Don. 16011.

\section{Family Crassulaceae}

Bryophyllum pinnatum (Lam.) Oken. 255, 3169, 3238, 3839.

Crassula aquatica (L.) Schönland. 6854.

Echeveria mucronata Schltdl. 29728bis, 29847.

Echeveria acutifolia Lindl. 15748.

Echeveria cuspidata Rose. 27831.

Echeveria elegans Rose. 19565, 20627, 22075, 22635, 22958, 24331, 24332, 25827, 27201, 27217, 27275.

Echeveria fulgens Lem. 2438.

Echeveria mucronata Schltdl. 28600.

Echeveria nodulosa (Baker) Otto. 26574, 26696, 26814.

Echeveria secunda Booth ex Lindl. 17342, 21878, 21879, 22193.

Echeveria strictiflora A. Gray. 16520, $22780,27529$.

Echeveria walpoleana Rose. 22356, 24578, 25151.

Kalanchoe delagoensis Ecklon \& Zeyh. 19998.

Lenophyllum latum Moran. 21695, 25639, 27777, 28178.

Sedum batesii Hemsl. 1689, 1731, 2735.

Sedum booleanum B.L. Turner. 20468 (TYPUS), 25688.

Sedum bourgaei Hemsl. 1901, 2132, 2931.

Sedum caducum R.T. Clausen. 25034, 25702, 25729.

Sedum calcicola B.L. Rob. \& Greenm. 18994, 20803, 21417, 24650, 24828, 27776, 28181.

Sedum chrysicaulum J.A. McDonald. 17260, 17303, 17919, 17977, 22416, 23971, 27215.

Sedum dendroideum Moc. \& Sessé ex DC. 530, 3227, 3551.

Sedum ebracteatum Moc. \& Sessé ex DC. 14933 (Type of Sedum ebracteatum subsp. grandifolium R.T. Clausen).

Sedum edwardsii (R.T. Clausen) B.L. Turner. 25143, 25147.

Sedum glabrum (Rose) Praeger. 25488.

Sedum gypsophilum B.L. Turner. 21596, 21654, 22462, 23591, 23768, 23860, 25157, 27811, 28095, 28116, 28138, 29004.

Sedum hintonii R.T. Clausen. 15926 (TYPUS).

Sedum hintoniorum B.L. Turner. 21297, 22455, 23797 (TYPUS), 23917, 23981, 25053.

Sedum jaliscanum S. Watson. 1886, 2011.

Sedum jurgensenii (Hemsl.) Moran. 18047.

Sedum longipes Rose. 3207, 3223.
Sedum macdonaldii G.L. Nesom. 18310, 19624, 27532.

Sedum moranense Kunth. 858.

Sedum nanifolium Frod. 25769.

Sedum palmeri S. Watson. 17806, 19116, 19287, 20190, 20221, 21809, 22596, 22740, 22776, 25296.

Sedum papillicaulum G.L. Nesom. 17551 (TYPUS), 22125, 22417, 27184.

Sedum pentastamineum R.T. Clausen. 8779.

Sedum praealtum A. DC. 21794.

Sedum reptans R.T. Clausen. 21142.

Sedum rhodocarpum Rose. 25124, 28570.

Sedum wrightii A. Gray. 16843, 17483, 18322, 18588, 19215, 19819, 19890, 20502, 20792, 21618, 21692, 22525, 23630, 23691, 23761, 23861, 23978, 24002, 25585, 25586, 27263, 27679, 28145, 28173, 28926, 29444.

Villadia aristata Moran. 17261, 17262, 17975, 20731, 22397, 22478, 23933, 23957, 23970, 27284, 27784, 27797, 29007.

Villadia cucullata Rose. 16841, 18594, 18929, 19858, 20720, 22566, 23531, 25996, 27122, 27241, 28693, 29039.

Villadia misera (Lindl.) R.T. Clausen. 20790, 22554.

Family Crossosomataceae

Forsellesia spinescens (A. Gray) Greene. 18167, 18706, 22609, 27011, 27667, 28983, 29914.

\section{Family Cucurbitaceae}

Cayaponia attenuata (Hook. \& Arn.) Cogn. 5196, 5203, 6123, 7282, 10875, 16226.

Citrullus lanatus (Thunb.) Matsum. \& Nakai. 5263, 17727, 27822.

Cucurbita argyrosperma K. Koch. 5491, 9682.

Cucurbita foetidissima Kunth. 16776, 18975, 19510, 27572.

Cucurbita pepo L. 21443, 21459.

Cyclanthera tamnoides (Willd.) Cogn. 29774.

Cyclanthera gracillima Cogn. 2219, 4666, 5020, 5165, 5266, $7010,8465,8540,12132,13199,19246,20838,23472$, 27704.

Cyclanthera langaei Cogn. 401, 1550, 2930bis, 8577, 15503, 22921, 23221, 23242, 23309, 24817.

Cyclanthera multifoliola Cogn. 10889, 13261.

Cyclanthera rostrata (P.G. Wilson) Kearns \& C.E. Jones. 2045 (TYPUS), 8458, 8616 (Type of Cyclanthera parviflora C.E. Jones ex Lira), 15528.

Cyclanthera tamnoides (Willd.) Cogn. 3433, 3438, 5041, 15532.

Echinopepon cirrhopedunculatus Rose. 8483, 9257, 10496. Echinopepon coulteri (A. Gray) Rose. 1968, 4963, 5004, 5211.

Echinopepon jaliscanus Rose. 10978, 11621, 14694.

Echinopepon racemosus (Steud.) C. Jeffrey. 8509.

Echinopepon wrightii (A. Gray) S. Watson. 6623, 26461.

Ibervillea lindheimeri (A. Gray) Greene. 23551.

Ibervillea tenuisecta (A. Gray) Small. 25767.

Lagenaria siceraria (Molina) Standl. 4951, 20524.

Luffa cylindrica (L.) M. Roem. 4778, 5710.

Melothria pendula L. 20568, 21212, 21552, 24633, 24792 , 24918. 
Melothria pringlei (S. Watson) Mart. Crov. 3734, 4030, 4583, 4695.

Microsechium palmatum (Ser.) Cogn. 1572, 6820, 6824, 14469, 26056, 26846, 26849.

Momordica charantia L. 5630, 10859, 14564, 26491.

Polyclathra albiflora (Cogn.) C. Jeffrey. 7319, 7334, 12475.

Polyclathra cucumerina Bertol. 12644.

Rytidostylis gracilis Hook. \& Arn. 14637, 16003.

Rytidostylis longisepala (Cogn.) C. Jeffrey. 1837, 1920, 2265, 5127, 5169, 13223.

Schizocarpum filiforme Schrad. 1778, 2071, 2138, 4750.

Schizocarpum longisepalum C. Jeffrey. 16169.

Schizocarpum palmeri Cogn. \& Rose. 9532, 10965, 11530, 12658, 26489.

Schizocarpum parviflorum B.L. Rob. \& Greenm. 8476, 9727, 13516.

Schizocarpum reflexum Rose. 5001.

Sechiopsis tetraptera Dieterle. 6624.

Sechiopsis triquetra (Moc. \& Sessé ex Ser.) Naudin. 2619, 5135, 6898, 8518, 8548.

Sechium hintonii (P.G. Wilson) C. Jeffrey. 4808, 5270, 8596 (TYPUS).

Sicyos barbatus (Gentry) C. Jeffrey. 12272.

Sicyos cordifolius Rodr.-Arévalo, Lira \& Dávila. 15407 (TYPUS).

Sicyos laciniatus L. 19701, 19775.

Sicyos longisepalus Cogn. 7362.

Sicyos microphyllus Kunth. 4882, 5058, 7026, 15529.

\section{Family Cunoniaceae}

Weinmannia pinnata L. 14238.

\section{Family Cyperaceae}

Bulbostylis capillaris (L.) C.B. Clarke. 1530, 2685.

Bulbostylis junciformis (Kunth) C.B. Clarke. 1732.

Bulbostylis juncoides (Vahl) Kük. ex Osten. 27913.

Bulbostylis pubescens (J. Presl \& C. Presl) Svenson. 1461, 1497, 4491, 4783, 6489, 9355.

Bulbostylis vestita (Kunth) C.B. Clarke. 4754.

Carex donnell-smithii L.H. Bailey. 7544.

Carex anisostachys Liebm. 4390.

Carex aztecica Mack. 9470.

Carex boliviensis van Heurck \& Müll. Arg. 14460.

Carex donnell-smithii L.H. Bailey. 3486.

Carex jamesonii Boott. 26757.

Carex planostachys Kunze. 20216, 28022.

Carex polystachya Sw. ex Wahlenb. 1833, 9883, 14726.

Carex potosina Hemsl. 16709.

Carex psilocarpa Steud. 3293.

Carex schiedeana Kuntze. 18136, 19661, 20230, 21069, 21828, 22717, 23903.

Carex spissa L.H. Bailey. 18239, 21860, 22723.

Carex standleyana Steyerm. 26412.

Cladium jamaicense Crantz. 17903, 21910.

Cyperus seslerioides Kunth. 29842.

Cyperus aggregatus (Willd.) Endl. 1782.

Cyperus amabilis Vahl. 9305.
Cyperus articulatus L. 5831.

Cyperus aschenbornianus Boeck. 1563.

Cyperus canus J. Presl \& C. Presl. 2996, 8885, 11747, 19940, 20917, 25306.

Cyperus compressus L. 5991.

Cyperus cuspidatus Kunth. 4762.

Cyperus distans L. f. 9496.

Cyperus esculentus L. 1153.

Cyperus flavescens L. 1359, 9432.

Cyperus hermaphroditus (Jacq.) Standl. 961.

Cyperus humilis Kunth. 5536.

Cyperus imbricatus Retz. 5818.

Cyperus ischnos Schltdl. 1826, 9465.

Cyperus laevigatus L. 6173.

Cyperus lanceolatus Poir. 9302.

Cyperus laxus Lam. 10792, 10972, 14645.

Cyperus macrostachyos Lam. 4685, 5454, 5815, 9460.

Cyperus manimae Kunth. 1367, 9244, 13112, 21127, 23715.

Cyperus niger Ruiz \& Pav. 15143.

Cyperus odoratus L. 2272, 8883.

Cyperus pallidicolor (Kük.) G.C. Tucker. 22049, 22115, 22506, 22922, 23092, 23454.

Cyperus panamensis (C.B. Clarke) Britton ex Standl. 11401.

Cyperus polystachyos Rottb. 6699.

Cyperus seslerioides Kunth. 1229, 6168.

Cyperus spectabilis Link. 9323.

Cyperus squarrosus L. 6475.

Cyperus surinamensis Rottb. 1067, 3732, 6038, 8884, 9689.

Cyperus tenerrimus J. Presl \& C. Presl. 1060, 6454, 9111, 10884, 12110, 14342.

Cyperus thyrsiflorus Jungh. 21407, 21462, 22216, 23719.

Cypringlea analecta (Beetle) M.T. Strong. 19231, 21875, 22037, 23337, 24238.

Eleocharis bella (Piper) Svenson. 2662.

Eleocharis geniculata (L.) Roem. \& Schult. 2161, 3307, 3340, 20326, 21483.

Eleocharis montevidensis Kunth. 21881.

Eleocharis mutata (L.) Roem. \& Schult. 1276.

Fimbristylis autumnalis (L.) Roem. \& Schult. 27698.

Fimbristylis complanata (Retz.) Link. 2245, 6039.

Fimbristylis cymosa R. Br. 5534.

Fimbristylis dichotoma (L.) Vahl. 1615, 9431, 10461, 12336.

Fuirena incompleta Nees. 4406.

Fuirena simplex Vahl. 5559, 5845, 20169, 29266.

Kyllinga odorata Vahl. 1176, 9429, 13103, 24896.

Lipocarpha micrantha (Vahl) G.C. Tucker. 5666, 8893.

Rhynchospora colorata (L.) H. Pfeiff. 17853, 20346, 20405, 23817, 25240.

Rhynchospora contracta (Nees) J. Raynal. 2538, 4996, 6630.

Rhynchospora kunthii Nees ex Kunth. 6143.

Rhynchospora nervosa (Vahl) Boeck. 1073, 1247, 9458, 14346.

Rhynchospora radicans (Schltdl. \& Cham.) H. Pfeiff. 
14696.

Rhynchospora robusta (Kunth) Boeck. 2160.

Rhynchospora schiedeana Kunth. 1349.

Scleria lithosperma (L.) Sw. 4759.

Scleria oligantha Michx. 23486.

Scleria reticularis Michx. 2243, 4511.

Scleria scabriuscula Schltdl. 1451, 9454.

Scleria tenella Kunth. 2184, 9463.

Schoenoplectus acutus (Muhl. ex Bigelow) A. Löve \& D. Löve. 16806.

Schoenoplectus californicus (C.A. Mey.) Soják. 17891, 21955.

Schoenus nigricans L. 21877, 22034.

Family Cytinaceae

Bdallophytum andrieuxii Eichler. 10134.

\section{Family Chloranthaceae}

Hedyosmum mexicanum Cordem. 586, 599, 3202, 3428, 3515, 3516, 3668, 8849, 14076.

\section{Family Chrysobalanaceae}

Conepia polyandra (Kunth) Rose. 13750, 14008, 14126.

Hirtella racemosa Lam. 10773, 11040.

Licania arborea Seem. 3832, 5957, 10015.

\section{Family Dilleniaceae}

Curatella americana L. 6597, 9996, 10009, 10205, 11736.

\section{Family Dioscoreaceae}

Dioscorea convolvulacea Schltdl. \& Cham. 1289, 4334, 10968.

Dioscorea dugesii B.L. Rob. 13266.

Dioscorea galeottiana Kunth. 15462, 15506, 17419bis.

Dioscorea guerrerensis R. Knuth. 1265, 1918, 4779, 6659

(TYPUS).

Dioscorea hintonii R. Knuth. 5008, 5215 (TYPUS), 8038.

Dioscorea insignis C.V. Morton \& B.G. Schub. 6713 (TYPUS), 6716, 11555, 14716.

Dioscorea jaliscana S. Watson. 11252.

Dioscorea liebmannii Uline. 11426.

Dioscorea longituba Uline. 806, 2703.

Dioscorea mitis C.V. Morton. 10585.

Dioscorea morelosana (Uline) Matuda. 6469.

Dioscorea nelsonii Uline ex R. Knuth. 11462.

Dioscorea palmeri R. Knuth. 4275.

Dioscorea pantojensis R. Knuth. 6226 (TYPUS).

Dioscorea platycolpota Uline. 15626.

Dioscorea plumifera B.L. Rob. 13263.

Dioscorea polygonoides Humb. \& Bonpl. ex Willd. 6625.

Dioscorea pringlei B.L. Rob. 1693, 2395.

Dioscorea remotiflora Kunth. 6694.

Dioscorea tancitarensis Matuda. 15513 (TYPUS).

Dioscorea temascaltepecensis R. Knuth. 2617, 2716 (TYPUS), 4304.

Dioscorea tubiperianthia Matuda. 8475 (TYPUS).

Dioscorea urceolata Uline. 5321, 17406bis.

Dioscorea uruapanensis Matuda. 15686 (TYPUS).

\section{Family Ebenaceae}

Diospyros ebenaster Retz. 3216.

Diospyros palmeri Eastw. 17738, 24207, 25319.

Diospyros rekoi Standl. 941, 5787, 7413.

Diospyros salicifolia Humb. \& Bonpl. ex Willd. 3959, 4127, 5568, 6044, 6117, 7058 .

\section{Family Ehretiaceae}

Bourreria hintonii I.M. Johnst. 10866 (TYPUS).

Bourreria huanita (Lex.) Hemsl. 12041.

Bourreria longiflora I.M. Johnst. 15908 (TYPUS).

Bourreria spathulata (Miers) Hemsl. 6310, 6488, 6541.

Bourreria superba I.M. Johnst. 10263, 13834 (TYPUS), 13905, 15880.

Ehretia anacua (Terán \& Berland.) I.M. Johnst. 24056, 24209, 25191.

Ehretia latifolia DC. 2837, 3419, 18199.

Lennoa madreporoides Lex. 9535.

Tiquilia gossypina (Wooton \& Standl.) A.T. Richardson. 29562.

Tiquilia canescens (DC.) A.T. Richardson. 16783, 18232, 20765, 21980, 23002, 25360, 25509, 25858, 25871, 27093, 29148.

Tiquilia gossypina (Wooton \& Standl.) A.T. Richardson. 24740, 28828, 28869, 28874, 29278, 29402.

Tiquilia greggii (Torr. \& A. Gray) A.T. Richardson. 16536, 23001, 23569, 24738, 28441, 28530, 29572.

Tiquilia hispidissima (Torr. \& A. Gray) A.T. Richardson. 28442, 29277, 29367.

Tiquilia mexicana (S. Watson) A.T. Richardson. 16570, 22867, 23199, 23560, 27479, 28249, 28415, 28527, 28531, 28632.

Tiquilia purpusii (Brandegee) A.T. Richardson. 23869, 27383, 27823.

Tiquilia tuberculata A.T. Richardson. 28802.

Tiquilia turneri A.T. Richardson. 28786.

\section{Family Ericaceae}

Agarista mexicana (Hemsl.) Judd. 3377, 3415, 3528, 3899, 4243, 7450, 14199, 14205.

Arbutus occidentalis McVaugh \& Rosatti. 8847 (Type of Arbutus occidentalis var. villosa McVaugh \& Rosatti).

Arbutus xalapensis Kunth. 882, 2743, 3695, 3858, 4387, 5916, 7351, 7666, 9928, 12817, 15556, 15566, 16757, 17028, 17377, 17518bis, 18161, 19374, 24085, 24947, 26205, 28084.

Arctostaphylos pungens Kunth. 16750, 16755, 17010, 18204, 18270, 18375, 20109, 21793, 21801, 21834, 22639, 26089, 26220.

Bejaria aestuans Mutis ex L. 9760, 9763, 9935, 11221, 14237 (Type of Bejaria hintonii Camp).

Bejaria mexicana Benth. 14106, 14200.

Comarostaphylis discolor (Hook.) Diggs. 3473, 3474, 13711, 14226, 14246, 14293, 26098, 26234, 26263, 26398, 26573. Comarostaphylis longifolia (Benth.) Klotzsch. 279, 2383, 3501, 5915, 5918, 8347.

Comarostaphylis polifolia (Kunth) Zucc. ex Klotzsch. 249, $275,887,6520,13556,16588,17070,17344,18217,18412$, 
18417, 18968, 19377, 22038, 22793, 23955, 24061, 27341, 27396, 28066.

Chimaphila maculata (L.) Pursh. 1871, 10726, 11068, 14204, 14406, 14501, 17540, 22929, 23236.

Chimaphila umbellata (L.) W.P.C. Barton. 14446, 17542, 22287.

Gaultheria erecta Vent. 507, 3424, 6055, 7356, 7548, 10153, 17175.

Lyonia squamulosa M. Martens \& Galeotti. 22933, 22982, 22991, 24030, 25177, 25183.

Monotropa hypopitys L. 861, 7710, 8566, 18340, 20442, 20509, 21624, 22430, 23973, 25093, 26659.

Monotropa uniflora L. 1708.

Orthilia secunda (L.) House. 1716, 18870, 18936, 19026, 23972.

Pernettya prostrata (Cav.) DC. 4000, 4254, 4927, 5084, 8325, 14292, 26149, 26580.

Pterospora andromedea Nutt. 3281, 4198, 20456, 20510.

Pyrola angustifolia (Alef.) Hemsl. 14457.

Vaccinium caespitosum Michx. 610, 4190, 14450.

Vaccinium confertum Kunth. 3466, 7389, 7538, 8285.

Vaccinium kunthianum Klotzsch. 17543, 22680, 22818, 22907, 22963, 24077, 24891.

\section{Family Eriocaulaceae}

Eriocaulon ehrenbergianum Klotzsch ex Körn. 627, 3488, $3638,4549$.

\section{Family Erythroxylaceae}

Erythroxylum mexicanum Kunth. 924, 3937, 4144, 6014, 7604, 7845, 9171, 10004, 10397, 10412, 13808, 13828. Erythroxylum rotundifolium Lunan. 10353.

\section{Family Euphorbiaceae}

Acalypha phleoides Cav. 18020.

Acalypha alopecuroidea Jacq. 6434, 10619, 14341, 15241.

Acalypha cincta Müll. Arg. 1063, 3960, 10238, 10285, 10577.

Acalypha dioica S. Watson. 17365, 20574, 21175, 21648, 23412, 24944, 25623, 25907, 28163.

Acalypha euphrasiostachys Bartlett. 1954.

Acalypha filipes (S. Watson) McVaugh. 4267, 5835, 6130, 13820, 13831.

Acalypha grisea Pax \& K. Hoffm. 984, 1157, 10411.

Acalypha havanensis Müll. Arg. 1487, 4342, 6482.

Acalypha hispida Burm. f. 15337.

Acalypha hypogaea S. Watson. 1757, 8072, 8086, 8134, 8149, 10572.

Acalypha macrostachya Jacq. 14730.

Acalypha microphylla Klotzsch. 12201, 26500.

Acalypha mollis Kunth. 26732.

Acalypha monostachya Cav. 20407, 20766, 22332, 23015, 28497, 28689.

Acalypha multiflora (Standl.) Radcl. Sm. 13818.

Acalypha ocymoides Kunth. 4405, 4596 (Type of Acalypha erecta Paul G. Wilson), 8732, 13936, 16030.

Acalypha phleoides Cav. 19597, 20343, 20374, 21236, 21404, 23072, 24297, 24611, 24686, 25583, 27556, 28978,
29027.

Acalypha polystachya Jacq. 1465, 1971, 4306, 4514, 4551, 6339, 10556.

Acalypha subterranea Paul G. Wilson. 8177 (TYPUS).

Acalypha trilaciniata Paul G. Wilson. 12026 (TYPUS).

Acalypha umbrosa Brandegee. 26421.

Acalypha vagans Cav. 8736, 10363, 10823, 11785, 12169, 12207, 13867.

Acalypha veronicoides Pax \& K. Hoffm. 1183.

Argythamnia tinctoria Millsp. 4731.

Astraea lobata (L.) Klotzsch. 3577, 4568, 5508.

Bernardia mexicana (Hook. \& Arn.) Müll. Arg. 3356, 3365, 6176, 6177, 10415, 11751.

Bernardia myricifolia (Scheele) S. Watson. 16618, 21466, 28144.

Caperonia palustris (L.) A. St.-Hil. 6494.

Cnidoscolus rostratus Lundell. 10113, 10130 (Type of Cnidoscolus rostratus subsp. hintonii Breckon).

Cnidoscolus spinosus Lundell. 10347.

Cnidoscolus angustidens Torr. 932, 934, 3962, 6134.

Cnidoscolus multilobus (Pax) I.M. Johnst. 13908 (Type of Cnidoscolus multilobus subsp. elasticoides Breckon ex Fern. Casas), 24193.

Cnidoscolus rostratus Lundell. 11984.

Cnidoscolus rotundifolius (Müll. Arg.) McVaugh. 24273, 24292.

Cnidoscolus tubulosus (Müll. Arg.) I.M. Johnst. 6268, 13793.

Cnidoscolus urens (L.) Arthur. 26482.

Croton ciliatoglandulifer Ortega. 19969.

Croton adspersus Benth. 1222, 1394, 11818, 13010.

Croton alamosanus Rose. 13824.

Croton arboreus Millsp. 25136.

Croton argyranthemus Michx. 17479.

Croton ciliatoglandulifer Ortega. 13879, 17597, 17599, 22955, 23287, 24098, 24196, 25131.

Croton cortesianus Kunth. 24150, 28160.

Croton dioicus Cav. 10610, 16614, 16738, 20610, 22333, 24469, 27728, 28961.

Croton draco Schltdl. \& Cham. 4540, 13930.

Croton fruticulosus Torr. 16894, 17642, 20051, 20111, 20548, 21166, 21382, 24358, 25617.

Croton hirtus L'Hér. 4316, 4463, 4552, 10574.

Croton hypoleucus Schltdl. 16519, 21999, 24463.

Croton incanus Kunth. 19956, 20802, 21150, 24149, 28017, 28622.

Croton monanthogynus Michx. 17694, 20332.

Croton morifolius Willd. 7860.

Croton pottsii (Klotzsch) Müll. Arg. 24739, 28866.

Croton repens Schltdl. 1017, 10514.

Croton roxanae Croizat. 1200.

Croton suaveolens Torr. 382, 19757, 25776, 27851.

Croton suberosus Kunth. 14552, 26467.

Croton tenuilobus S. Watson. 4293, 12690.

Croton xalapensis Kunth. 13806.

Chiropetalum astroplethos (J.W. Ingram) Radcl. Sm. \& R. Govaerts. 21091, 22771.

Chiropetalum schiedeanum (Müll. Arg.) Pax. 3511, 4945, 
6853, 17510, 22645.

Dalechampia scandens L. 4769, 10923, 10935.

Dalembertia populifolia Baill. 4353, 4747, 6618, 6766, 13306.

Ditaxis guatemalensis (Müll. Arg.) Pax \& K. Hoffm. 3618, 5438,6600 .

Ditaxis humilis (Engelm. \& A. Gray) Pax. 24735, 28884.

Ditaxis micrandra (Croizat) Radcl. Sm. \& Govaerts. 6307 (TYPUS).

Euphorbia ariensis Kunth. 9549.

Euphorbia greggii Engelm. ex Boiss. 25928.

Euphorbia indivisa (Engelm.) Tidestr. 29765.

Euphorbia oaxacana B.L. Rob. \& Greenm. 11308.

Euphorbia adenoptera Bertol. 2199, 2405, 2998, 16252.

Euphorbia albomarginata Torr. \& A. Gray. 24758.

Euphorbia antisiphylitica Zucc. 17521bis, 19652, 21780, 23044, 23198, 28365.

Euphorbia anychioides Boiss. 3317, 4909.

Euphorbia ariensis Kunth. 4706, 9413, 12183, 15549.

Euphorbia arizonica Engelm. 28383.

Euphorbia beamanii M.C. Johnst. 17003, 17345, 20148, 20202, 20987, 22577.

Euphorbia bifurcata Engelm. 23501, 27273, 28158.

Euphorbia calyculata Kunth. 1524, 4629, 8264, 13953, 15129.

Euphorbia capitellata Engelm. 16649.

Euphorbia cinerascens Engelm. 20229, 23856, 24256, 25713, 25789, 28036, 28663.

Euphorbia coalcomanensis (Croizat) V.W. Steinm. 12685, 15130, 15765 (TYPUS).

Euphorbia cotinifolia L. 2031, 4973, 10720.

Euphorbia crepitata L.C. Wheeler. 29401.

Euphorbia cumbrae Boiss. 4775, 9357, 23723, 24281, 25657 , 28335.

Euphorbia cyathophora Murray. 13222, 24276.

Euphorbia cymbifera (Schltdl.) V.W. Steinm. 13772.

Euphorbia delicatula Boiss. 2597, 5202, 6925.

Euphorbia dentata Michx. 1339, 4672, 23396, 27245, 27908, 28195, 28496.

Euphorbia dioeca Kunth. 26505.

Euphorbia dioscoreoides Boiss. 1751, 1787, 4840, 6924, 8366, 9629, 13132, 15196, 15327.

Euphorbia dressleri V.W. Steinm. 10973 (TYPUS).

Euphorbia eriantha Benth. 28347, 28475, 29235.

Euphorbia esuliformis S. Schauer ex Nees \& S. Schauer. 8402.

Euphorbia francoana Boiss. 1635, 6595, 9514, 11442, 13333, 15199, 16119.

Euphorbia fruticulosa Engelm. ex Boiss. 22869, 28307, 28354, 28395, 28769, 28825, 28875, 29428, 29439.

Euphorbia furcillata Kunth. 497, 3994, 19916, 21815, 21895, 22253, 22496, 22612, 22836, 24065, 24076, 24139, 24163, $24311,24389,24584,25944,27850,27950,28082,28581$, 28609 .

Euphorbia graminea Jacq. 1772, 1872, 1914, 2246, 2382, 2478, 3109, 4770, 4837, 4937, 5101, 7262, 9792, 10840, 10971, 11535, 12625, 13321, 13955, 15297, 21662, 23393, 23475.
Euphorbia greggii Engelm. ex Boiss. 20235, 20274, 21388, 23332, 23898, 25571, 25590, 25612, 25695, 25929.

Euphorbia heterophylla L. 1784, 1995, 2940, 4667, 4673, 5024, 5186, 6636, 11576, 12068, 13227, 13247, 14550, 14616, 15197, 15219.

Euphorbia hintonii L.C. Wheeler. 4534, 6092, 6257 (TYPUS), 6351.

Euphorbia hirta L. 195, 557, 757, 4959, 5464, 5544bis, 5870, 9186, 9786, 10909, 12864.

Euphorbia hypericifolia L. 687, 765, 836, 1479, 2404, 3869, 13603, 14551.

Euphorbia hyssopifolia L. 10562, 11434.

Euphorbia lata Engelm. 24769.

Euphorbia leucocephala Lotsy. 9378, 9406.

Euphorbia linguiformis McVaugh. 6486, 12014 (TYPUS).

Euphorbia longecornuta S. Watson. 22772.

Euphorbia macropus (Klotzsch \& Garcke) Boiss. 1552, 4652, 22378, 23392, 23403, 23674, 23682, 24497, 25467 , 27272.

Euphorbia maculata L. 13871, 15298.

Euphorbia mcvaughiana M.C. Johnst. 20106, 25806, 27179.

Euphorbia mendezii Boiss. 23037, 27614.

Euphorbia micromera Boiss. 28333, 28731, 28814.

Euphorbia milii Des Moul. 6536, 8585.

Euphorbia montereyana Millsp. 22896, 24592, 24683, 24795.

Euphorbia multiseta Benth. 4842, 11484, 11636, 15385.

Euphorbia neilmulleri M.C. Johnst. 18170, 18387, 20189, 20948, 21861, 22095, 22699, 23516, 23641, 24243, 27726.

Euphorbia nutans Lag. 20336, 21426, 23417, 23520, 25854.

Euphorbia oaxacana B.L. Rob. \& Greenm. 7577, 10399, 15768.

Euphorbia ocymoidea L. 2406, 2945, 5100.

Euphorbia peritropoides (Millsp.) V.W. Steinm. 9955, 10255, 11058, 11187, 14004, 14991.

Euphorbia pulcherrima Willd. ex Klotzsch. 11722, 15421, 15799 .

Euphorbia radians Benth. 666, 3339, 3483, 7152.

Euphorbia revoluta Engelm. 23557.

Euphorbia schiedeana (Klotzsch \& Garcke) Mayfield. 15469, 25674, 25698.

Euphorbia schlechtendalii Boiss. 3758, 7575.

Euphorbia segoviensis (Klotzsch \& Garcke) Boiss. 10907.

Euphorbia serpens Kunth. 5449, 16680.

Euphorbia serpyllifolia Pers. 5548, 21109, 27212, 28492, 28648, 28813.

Euphorbia spathulata Lam. 1029.

Euphorbia sphaerorhiza Benth. 962, 3887, 4070.

Euphorbia stictospora Engelm. 5515, 19004, 19512, 19531, 19582, 24855.

Euphorbia subpeltata S. Watson. 24852.

Euphorbia subreniformis S. Watson. 8513.

Euphorbia succedanea L.C. Wheeler. 6580 (TYPUS).

Euphorbia tanquahuete Sessé \& Moc. 731, 3822, 7731, 8087, 8237, 8599, 10184, 10392.

Euphorbia tithymaloides L. 10331. 
Euphorbia umbellulata Engelm. ex Boiss. 743, 15185, 15290.

Euphorbia velleriflora (Klotzsch \& Garcke) Boiss. 5174, 20764.

Euphorbia vestita Boiss. 13229, 14419.

Euphorbia villifera Scheele. 18470, 19851, 20382, 20826, 21454, 21603, 22858, 23334, 23839, 24464, 27244, 28907.

Euphorbia whitei L.C. Wheeler. 5240, 26318.

Gymnanthes actinostemoides Müll. Arg. 8106.

Hura polyandra Baill. 3979, 4063, 14417.

Jatropha curcas L. 4300 (Type of Jatropha hintonii Wilbur), 9100, 10362.

Jatropha dioica Sessé ex Cerv. 16610, 20921, 22790, 25825, 28348.

Jatropha macrorhiza Benth. 16717.

Jatropha platyphylla Müll. Arg. 13785, 13938.

Jatropha riojae Miranda. 1485.

Jatropha rufescens Brandegee. 3769, 7706, 9959.

Manihot aesculifolia (Kunth) Pohl. 1207, 4374, 4555, 6264, 6468, 10376.

Manihot angustiloba (Torr.) Müll. Arg. 940, 4349, 4467, 6188.

Manihot chlorosticta Standl. \& Goldman. 10345, 10939.

Manihot esculenta Crantz. 4698, 7044, 13972.

Manihot rhomboidea Müll. Arg. 4220, 4437, 8029, 9090, 10488, 15072.

Manihot subspicata D.J. Rogers \& Appan. 24255.

Manihot triloba (Sessé ex Cerv.) McVaugh ex Miranda. 4149.

Ricinus communis L. 4811, 5481, 5973.

Sapium macrocarpum Müll. Arg. 14548.

Sebastiania hintonii Lundell. 14743 (TYPUS).

Stillingia sanguinolenta Müll. Arg. 24955.

Stillingia zelayensis (Kunth) Müll. Arg. 1024, 1127, 4409, 10511, 17214.

Tragia affinis B.L. Rob. \& Greenm. 4802, 5028.

Tragia nepetifolia Cav. 1292, 4435, 4562, 5790, 10621, $18969,25474$.

\section{Family Fabaceae}

Acaciella angustissima (Mill.) Britton \& Rose. 1284, 4744, 5367, 5377, 5688, 7162, 7709, 7778, 7963, 8016, 8021, 8027, $8142,8183,8184,8621,8724,8780,9489,10538,10896$, $12415,12495,14857,14967,14968,15467,18012,21159$, 24103, 24562, 24656, 26328, 26483.

Acaciella hartwegii (Benth.) Britton \& Rose. 1291, 1423, 9418.

Acaciella igualensis Britton \& Rose. 2500, 5412, 8136.

Acaciella painteri Britton \& Rose. 8763, 8856, 9097, 12453.

Acaciella sousae (L. Rico) L. Rico. 9541 (TYPUS).

Acaciella tequilana (S. Watson) Britton \& Rose. 1945, 4852, 5405.

Acaciella villosa (Sw.) Britton \& Rose. 10815, 17233.

Acmispon oroboides (Kunth) Brouillet. 2422, 3422, 4877, 5226, 8733.

Adenopodia gymnantha Brenan. 12588 (TYPUS).

Aeschynomene americana L. 1619, 1984, 2057, 2234, 5992,
6654, 8608, 9503, 9668, 9669, 10747, 10922, 11604, 11670, 12915, 13287, 14653, 15243.

Aeschynomene compacta Rose. 7872.

Aeschynomene elegans Schltdl. \& Cham. 25051.

Aeschynomene hintonii Sandwith. 6104 (TYPUS), 10644.

Aeschynomene hystrix Poir. 4582.

Aeschynomene palmeri Rose. 16060.

Aeschynomene paniculata Willd. ex Vogel. 5044, 11396.

Aeschynomene paucifoliolata Micheli. 1764, 1997, 4784, 6708, 6938, 7059.

Aeschynomene petraea B.L. Rob. 10395, 11332, 12950, 13804.

Aeschynomene rudis Benth. 16163.

Aeschynomene sensitiva Sw. 1930, 4957, 6679.

Aeschynomene unijuga (M.E. Jones) Rudd. 2410.

Aeschynomene villosa Poir. 25049.

Aeschynomene virginica (L.) Britton, Stearn \& Pogg. 9667, 11662.

Albizia tomentosa (Micheli) Standl. 3815, 3966, 6046, 9980, 10326.

Amicia zygomeris DC. 808, 1816, 2047, 2554, 5087, 7012, 9573, 11482, 12267, 13352, 14874, 15458, 21233, 24599.

Andira inermis (W. Wright) Kunth ex DC. 3805, 5877, 9978, 14091.

Apoplanesia paniculata C. Presl. 10856, 12116, 12606, 16034.

Arachis hypogaea L. 3741.

Astragalus hypoleucus S. Schauer. 18064.

Astragalus micranthus Desv. 17995.

Astragalus coahuilae M.E. Jones. 18381, 18800, 25849, 25976.

Astragalus coriaceus Hemsl. 21912.

Astragalus emoryanus (Rydb.) Cory. 21931.

Astragalus ervoides Hook. \& Arn. 12727.

Astragalus greggii S. Watson. 18164, 24374.

Astragalus guatemalensis Hemsl. 5067, 5232, 8912, 15465.

Astragalus hintonii Barneby. 13240 (TYPUS).

Astragalus hypoleucus S. Schauer. 17398, 17864, 18521, 19254, 20131, 20577, 21902, 21944, 22015, 23101, 23679, 25407, 27050.

Astragalus legionensis Barneby. 17382, 18871, 18937.

Astragalus mollissimus Torr. 17770, 18548, 19128, 19593, 19736, 21901, 25364.

Astragalus nothoxys A. Gray. 25335.

Astragalus nuttallianus DC. 18184, 29087, 29095.

Astragalus parvus Hemsl. 29393.

Astragalus purpusii M.E. Jones. 17000, 17202, 17293, 17328, 18830, 19121, 19397, 20207, 20458, 20980, 22076, 27541. Astragalus quinqueflorus S. Watson. 18182, 18831.

Astragalus racemosus Pursh. 18372.

Astragalus sanguineus Rydb. 18799, 21857, 24375, 24425, 25353, 25435.

Astragalus scalaris S. Watson. 29171.

Astragalus strigulosus Kunth. 12914.

Astragalus tolucanus B.L. Rob. \& Seaton. 1900.

Ateleia arsenii Standl. 407, 3421, 3526, 4240, 5286, 6093, 6158, 7621.

Bauhinia bartlettii B.L. Turner. 25213. 
Bauhinia divaricata L. 10959, 11655, 12085, 12289, 12537 , 12559, 16113, 24513, 24622, 24780.

Bauhinia lunarioides A. Gray ex S. Watson. 16796, 16802, 24051, 27061.

Bauhinia macranthera Benth. ex Hemsl. 17789, 18402, 24230, 24323, 25915.

Bauhinia pauletia Pers. 1990, 5197, 5691, 6619.

Bauhinia pringlei S. Watson. 3579, 5384, 5738.

Bauhinia purpurea L. 15743.

Bauhinia ramosissima Benth. ex Hemsl. 16527, 18262, 19098, 21937, 21992, 22033, 22727, 22878, 23011, 23363, 25744, 25746, 28278, 28301, 29138.

Bauhinia subrotundifolia Cav. 15854, 16217.

Bauhinia ungulata L. 2987, 5297, 6945, 16240.

Brongniartia intermedia Moric. 29836.

Brongniartia cuneata L.B. Sm. \& B.G. Schub. 6311 (TYPUS), 8019, 8727.

Brongniartia foliolosa Benth. ex Hemsl. 21267, 22941, 23474.

Brongniartia funiculata L.B. Sm. \& B.G. Schub. 1591, 5397 (TYPUS).

Brongniartia glabrata Hook. \& Arn. 11619.

Brongniartia inconstans S. Watson. 10035.

Brongniartia intermedia Moric. 870, 890, 6230, 17083, 20369, 22735, 22807.

Brongniartia lupinoides (Kunth) Taub. 4067, 5926, 11973.

Brongniartia podalyrioides Kunth. 5387, 5854, 9831, 10223.

Brongniartia proteranthera L.B. Sm. \& B.G. Schub. 5947 (TYPUS), 7750.

Brongniartia vicioides M. Martens \& Galeotti. 10435.

Caesalpinia pulcherrima (L.) Sw. 733, 945, 4594, 5104, 5875, 6666, 6955, 10049.

Cajanus cajan (L.) Millsp. 15761.

Calopogonium caeruleum (Benth.) C. Wight. 3134, 5584, 5829, $11645,16281$.

Calopogonium galactioides (Kunth) Benth. ex Hemsl. 5404, 5579, 12912.

Calliandra bijuga Rose. 7806, 12726, 13673.

Calliandra caeciliae Harms. 6317.

Calliandra eriophylla Benth. 16524, 16860, 17654, 19714, 21024, 21423, 21498, 21544, 22028, 23063, 23854, 25855, 28541 .

Calliandra grandiflora (L'Hér.) Benth. 903, 1545, 10503, 11877, 12712, 13858, 13958, 14808, 15384.

Calliandra hintonii Barneby. 4099 (TYPUS).

Calliandra hirsuta (G. Don) Benth. 12711.

Calliandra houstoniana (Mill.) Standl. 6772, 6967, 10685, 11044, 11457, 14562, 15553.

Calliandra pityophila Barneby. 14377 (TYPUS).

Calliandra sinaloana (Britton \& Rose) Standl. 10183.

Calliandra tergemina (L.) Benth. 13775, 13810, 13931, 14128bis.

Canavalia brasiliensis Mart. ex Benth. 5341, 6910, 11618, 12237, 26473.

Canavalia hirsutissima J.D. Sauer. 11053 (TYPUS).

Canavalia septentrionalis J.D. Sauer. 17637, 22145, 24316, 24807.
Canavalia villosa Benth. 2082, 2288, 8875, 9911, 10826, 11673, 11860, 14037, 15692, 16263.

Cassia grandis L. f. 5905, 6419, 7156, 7598.

Cassia hintonii Sandwith. 3440 (TYPUS), 5570, 7727, 15876.

Cenostigma eriostachys (Benth.) E. Gagnon \& G.P. Lewis. 5659, 6929, 16216, 16224.

Centrosema plumieri (Turpin ex Pers.) Benth. 3211, 3212, 10028, 11038.

Centrosema pubescens Benth. 2214, 7072, 10957, 16154, 16222.

Centrosema sagittatum (Willd.) Brandegee ex L. Riley. 5006, 10825, 10914, 11560.

Centrosema virginianum (L.) Benth. 4792, 4994, 7060, 9623, 17677, 24218, 24649.

Cercis canadensis L. 16735, 16935, 17051, 17517, 19353, 20133, 22756, 25951, 28078.

Cleobulia crassistyla R.H. Maxwell. 9900, 10791, 14996 (TYPUS).

Clitoria mexicana Link. 4968, 9892, 10751, 11668 (TYPUS).

Clitoria polystachya Benth. 1371, 1528, 4447 (Type of Clitoria polystachya var. congesta Fantz), 4523, 5334, 8001, $8125,8597,15073$.

Cojoba arborea (L.) Britton \& Rose. 14157.

Cologania broussonetii (Balb.) DC. 29848.

Cologania angustifolia Kunth. 19153, 21040, 22847, 25412, 25609, 27188.

Cologania biloba (Lindl.) G. Nicholson. 1580, 9587, 12204.

Cologania broussonetii (Balb.) DC. 10656, 11483, 11837, 13115, 15476, 17616, 18019, 21277, 24663, 25325, 25560.

Cologania hintoniorum B.L. Turner. 11262, 11694, 12767 (TYPUS), 14947.

Cologania obovata Schltdl. 751, 6069, 10394.

Cologania procumbens Kunth. 1023, 4366, 10305, 10444.

Conzattia multiflora (B.L. Rob.) Standl. 7793.

Coursetia caribaea (Jacq.) Lavin. 5191, 8490, 8497, 11463.

Coursetia glandulosa A. Gray. 3350, 5528, 5663, 5836.

Coursetia hintonii Rudd. 7051 (TYPUS).

Coursetia insomniifolia Lavin. 29302.

Coursetia madrensis Micheli. 14263.

Coursetia mollis B.L. Rob. \& Greenm. 2983, 3404, 15920, 16225.

Crotalaria angulata Mill. 2454, 10755.

Crotalaria bupleurifolia Cham. \& Schltdl. 1730, 2686 (Type of Crotalaria pilosa var. robusta H. Senn), 4595, 5068 (Type of Crotalaria tuerckheimii var. macrantha H. Senn), 5424, 5580, 11708, 12862.

Crotalaria cajanifolia Kunth. 1290.

Crotalaria filifolia Rose. 1474, 4717, 4835, 6498, 8176, 9464, 24586, 24666.

Crotalaria incana L. 4798, 6744, 24841.

Crotalaria longirostrata Hook. \& Arn. 1421, 8306, 10920, $11357,12725,12856,13055,14426,16157,26324,26628$, 26728.

Crotalaria maypurensis Kunth. 1626, 4854, 6757, 9563, 
$12238,12638,13126,26465$.

Crotalaria micans Link. 670, 10617, 10912, 12252, 12723, 12911.

Crotalaria mollicula Kunth. 4964, 12858, 15055, 15598.

Crotalaria polyphylla L. Riley. 11413.

Crotalaria pumila Ortega. 1636, 2059, 2652, 4658, 5540, 6041, 8291, 10902, 11395, 11580, 12198, 13145, 14560.

Crotalaria sagittalis L. 1780, 5185, 13888, 17451 bis, 22587 , 22619, 23231, 24696, 26061, 26171.

Crotalaria vitellina Ker Gawl. 1870, 9261, 10352, 10754, 10843, 11581, 14582, 17225.

Cynometra oaxacana Brandegee. 10335, 10893.

Chaetocalyx scandens (L.) Urb. 26471.

Chamaecrista absus (L.) H.S. Irwin \& Barneby. 2285, 4834, 5053.

Chamaecrista desvauxii (Collad.) Killip. 11192.

Chamaecrista flexuosa (L.) Greene. 4575, 6122, 9070, 10000.

Chamaecrista glandulosa (L.) Greene. 10698, 12708.

Chamaecrista greggii (A. Gray) Pollard ex A.H. Heller. 17937, 20922, 21229, 23872, 24261, 24618.

Chamaecrista kunthiana (Schltdl. \& Cham.) H.S. Irwin \& Barneby. 11388.

Chamaecrista nictitans (L.) Moench. 1766, 4663, 5972, 6614, 9660, 10688, 10841, 11385.

Chamaecrista punctulata (Hook. \& Arn.) H.S. Irwin \& Barneby. 5037, 7029, 9543, 11019, 11591.

Chamaecrista rotundifolia (Pers.) Greene. 715, 1593, 1864, 6754, 15239.

Chamaecrista serpens (L.) Greene. 1656, 9542, 10536. Chloroleucon mangense (Jacq.) Britton \& Rose. 11647. Dalbergia calycina Benth. 591, 881, 3971, 6194, 7931.

Dalbergia glomerata Hemsl. 3177, 4015, 5577, 7248.

Dalbergia melanocardium Pittier. 761, 3935, 4322.

Dalea leporina (Aiton) Bullock. 29850.

Dalea obovatifolia Ortega. 18021, 29739.

Dalea sericea Lag. 29731, 29869.

Dalea zimapanica S. Schauer. 29796.

Dalea bicolor Humb. \& Bonpl. ex Willd. 2327, 11840, 13397, 19763, 20623, 20633, 23776, 25687, 25917, 27688, 28015 .

Dalea botterii (Rydb.) Barneby. 17437, 22454, 23299, 23941.

Dalea brachystachys A. Gray. 20611.

Dalea capitata S. Watson. 17183, 17444, 18433, 19705, 19738, 21387, 23354, 28114, 28696.

Dalea carthagenensis (Jacq.) J.F. Macbr. 7564, 8572.

Dalea cliffortiana Willd. 1630, 2001, 2217, 2615, 2636, 4755, 5184, 5292, 6968, 9391, 9602, 9909, 11501, 12234, 13269, 15152.

Dalea cuniculo-caudata Paul G. Wilson. 1590, 2168, 2333 (TYPUS), 13462.

Dalea elata Hook. \& Arn. 2007bis, 5364, 15420, 15780.

Dalea eriophylla S. Watson. 17313, 18114, 18781, 19091, 19314, 28224, 28230.

Dalea filiciformis B.L. Rob. \& Greenm. 18169, 20931, 22696, 23746, 23857, 23992, 27372, 28593.

Dalea foliolosa (Aiton) Barneby. 1943, 2257, 6683, 7580,
9530, 9586, 12322, 12780.

Dalea frutescens A. Gray. 16517, 25406.

Dalea greggii A. Gray. 16723, 18382, 19111, 19284, 20847, 21755, 22027, 23845, 27905.

Dalea gypsophila Barneby. 18231, 19527, 27654.

Dalea hintonii Sandwith. 5314 (TYPUS), 8872.

Dalea hospes (Rose) Bullock. 16892, 18711, 27631, 28736. Dalea humilis G. Don. 1960, 2067, 2617bis, 2881, 5030, 5110.

Dalea laniceps Barneby. 27893.

Dalea leporina (Aiton) Bullock. 1686, 1888, 2273, 5048, 7786, 9665, 11992, 14715, 14823, 15579.

Dalea leucostachya A. Gray. 2007, 2086, 11549, 12401, 12777, 14926, 15554.

Dalea lutea (Cav.) Willd. 975, 1846, 16893, 17632, 18105, 18280, 18989, 21335, 23506.

Dalea melantha S. Schauer. 16736, 16761, 17289, 18383, 19317, 19821, 21991, 22779, 22999, 23355, 29449.

Dalea nana Torr. ex A. Gray. 29248.

Dalea neomexicana (A. Gray) Cory. 23567, 28304, 28513.

Dalea obovatifolia Ortega. 5317, 15463, 23762.

Dalea obreniformis (Rydb.) Barneby. 4982, 8352, 15561.

Dalea plantaginoides Barneby. 15665 (TYPUS).

Dalea pogonathera A. Gray. 22872, 23578, 25894, 27515.

Dalea polygonoides A. Gray. 28484.

Dalea polystachya (Sessé \& Moc.) Barneby. 5340.

Dalea prostrata Ortega. 19079.

Dalea radicans S. Watson. 20226.

Dalea reclinata (Cav.) Willd. 4880.

Dalea saffordii (Rose) Bullock. 17757, 19299, 19359, 20110, 21798, 21900, 22715, 22726, 28683.

Dalea sericea Lag. 1944, 13425, 14772.

Dalea tomentosa (Cav.) Willd. 2232, 9598, 11527, 15585.

Dalea uniflora (Barneby) G.L. Nesom. 18752, 18961, 21966, 21971, 28028, 28094, 28222, 28223, 28232.

Dalea urceolata Greene. 13351 (Type of Petalostemon tripetalus Paul G. Wilson).

Dalea versicolor Zucc. 12929 (Type of Dalea versicolor var. decipiens Barneby).

Dalea wrightii A. Gray. 16872, 21146, 23576, 25972, 27417 , 28783, 28871, 29123, 29246.

Delonix regia (Bojer ex Hook.) Raf. 723, 3604, 3616, 3754 , 4132, 5939.

Denisophytum sessilifolium (S. Watson) E. Gagnon \& G.P. Lewis. 24737.

Dermatophyllum juanhintonianum (B.L. Turner) B.L. Turner. 23025, 25154, 25155, 29142.

Dermatophyllum purpusii (Brandegee) Vincent. 29124.

Dermatophyllum secundiflorum (Ortega) Gandhi \& Reveal. 16597, 18966, 19319, 19383, 19648, 21987, 24981, 25153, 25734, 25743, 27385, 27729.

Desmanthus interior (Britton \& Rose) Bullock. 4795, 6885, 8218.

Desmanthus painteri (Britton \& Rose) Standl. 29155.

Desmanthus pringlei (Britton \& Rose) F.J. Herm. 24225.

Desmanthus virgatus (L.) Willd. 5440.

Desmodium grahamii A. Gray. 29726.

Desmodium uncinatum (Jacq.) DC. 18007, 29725. 
Desmodium affine Schltdl. 6717, 15245.

Desmodium alamanii DC. 8297.

Desmodium amplifolium Hemsl. 11092.

Desmodium angustifolium (Kunth) DC. 1611, 2235, 2301, 2505, 4492, 4827, 9610, 11498.

Desmodium barbatum (L.) Benth. 4952, 14625.

Desmodium bellum (S.F. Blake) B.G. Schub. 2920.

Desmodium caripense (Kunth) G. Don. 20552, 21436, 23425, 23831, 24658, 24890.

Desmodium cinereum (Kunth) DC. 9657, 10918, 13249.

Desmodium cordistipulum Hemsl. 2818, 15472.

Desmodium densiflorum Hemsl. 271, 2834, 8939.

Desmodium distortum (Aubl.) J.F. Macbr. 8363, 8573, 14864.

Desmodium ghiesbreghtii Hemsl. 1734.

Desmodium glutinosum (Muhl. ex Willd.) Wood. 23296.

Desmodium grahamii A. Gray. 19182, 21279, 23683, 24664, 25463, 25543.

Desmodium hartwegianum Hemsl. 1621.

Desmodium hookerianum D. Dietr. 10940, 11686.

Desmodium infractum DC. 1496, 4749, 6626.

Desmodium intortum (Mill.) Urb. 8575, 10944, 11524, 16130, 16131.

Desmodium jaliscanum S. Watson. 2020, 2528, 6771, 13395.

Desmodium lindheimeri Vail. 20556, 20589, 21392, 21545, 24654, 24812, 24837.

Desmodium macrostachyum Hemsl. 12159.

Desmodium michelianum (Schindl.) B.G. Schub. \& McVaugh. 2706.

Desmodium michoacanum B.G. Schub. \& McVaugh. 12923 (TYPUS).

Desmodium molliculum (Kunth) DC. 15473.

Desmodium neomexicanum A. Gray. 1927, 2174.

Desmodium nicaraguense Oerst. 2523, 5337, 8568.

Desmodium orbiculare Schltdl. 1055, 9258.

Desmodium plicatum Schltdl. \& Cham. 3000, 10828, 11638, 12843.

Desmodium prehensile Schltdl. 9661, 11031, 11511.

Desmodium pringlei S. Watson. 1894.

Desmodium procumbens (Mill.) Hitchc. 1754, 1928, 5187, 6693, 6695, 8494, 8517, 8729, 9534bis, 10863, 13303, 15244.

Desmodium psilophyllum Schltdl. 17606, 17960, 19163, 20578, 21384, 22897, 23173, 24228, 24305, 24615, 24655, 24871, 24907.

Desmodium retinens Schltdl. 19183, 21278, 22985, 24576.

Desmodium scorpiurus (Sw.) Desv. 3945, 5629, 6037, 11419, 16276.

Desmodium sericophyllum Schltdl. 11458, 12177.

Desmodium skinneri Benth. ex Hemsl. 2942, 3236, 5290.

Desmodium strobilaceum Schltdl. 9390, 9568.

Desmodium subrosum G.L. Nesom. 21133.

Desmodium sumichrastii (Schindl.) Standl. 10681, 11144, 12771, 26335, 26416.

Desmodium tortuosum (Sw.) DC. 1950, 5480, 7752, 8523, 8728, 11448, 17687.

Diphysa americana (Mill.) M. Sousa. 5221, 7322, 11755.
Diphysa floribunda Peyr. 11269, 12948, 13671, 14158, 15785.

Diphysa macrocarpa Standl. 1015.

Diphysa minutifolia Rose. 5796, 10031.

Diphysa puberulenta Rydb. 12928, 16205.

Diphysa racemosa Rose. 194, 312, 2975, 3011, 8877, 13564.

Diphysa suberosa S. Watson. 718, 770, 14196.

Ebenopsis ebano (Berland.) Barneby \& J.W. Grimes. 24154, 24334.

Entadopsis polystachya (L.) Britton. 925, 1478, 4274, 4781, 5945, 9148, 11379.

Enterolobium cyclocarpum (Jacq.) Griseb. 3416, 3641, 5788.

Eriosema diffusum (Kunth) G. Don. 1592.

Eriosema grandiflorum (Schltdl. \& Cham.) G. Don. 2495, 5106, 5293, 9797, 12470.

Eriosema longicalyx Grear. 8935.

Eriosema multiflorum B.L. Rob. 1988.

Eriosema palmeri S. Watson. 13124.

Eriosema pulchellum (Kunth) G. Don. 1181, 10462, 10844.

Erythrina americana Mill. 25266.

Erythrina herbacea L. 22758.

Erythrina sierra G.L. Nesom. 24307.

Erythrina breviflora DC. 527, 1861, 1924, 11513, 12314, 12468, 13382, 15603.

Erythrina lanata Rose. 3397, 10030, 12084, 13825.

Erythrina leptorhiza Moc. \& Sessé ex DC. 15402, 17163.

Erythrina mexicana Krukoff. 5333 (TYPUS).

Erythrina rubrinervia Kunth. 6157.

Erythrostemon acapulcensis (Standl.) E. Gagnon \& G.P. Lewis. 10991.

Erythrostemon caladenia (Standl.) E. Gagnon \& G.P. Lewis. 16198, 16228.

Erythrostemon gilliesii (Wall. ex Hook.) Klotzsch. 16774, 17533.

Erythrostemon hintonii (Sandwith) Gagnon \& G.P. Lewis. 5567, 7746 (TYPUS).

Erythrostemon mexicanus (A. Gray) Gagnon \& G.P. Lewis. 16876, 17624, 17744, 17785, 21225, 22183, 24177, 24620, 24712, 25218.

Eysenhardtia parvifolia Brandegee. 16511, 21985.

Eysenhardtia polystachya (Ortega) Sarg. 380, 1143, 1223, 8015, 9247, 12738, 13060, 15172, 22343.

Eysenhardtia texana Scheele. 16590, 16804, 17969, 20608, 20857, 21177, 22463, 23050, 23137, 24398.

Galactia acapulcensis Rose. 8064, 8220.

Galactia brachystachya Benth. 25866.

Galactia canescens Benth. 29062.

Galactia incana (Rose) Standl. 1652, 10476, $11378,15053$. Galactia multiflora B.L. Rob. \& Greenm. 1180, 6559.

Galactia striata (Jacq.) Urb. 4829, 5388, 11409, 11412.

Galactia viridiflora (Rose) Standl. 5389, 6893, 13375.

Galactia wrightii A. Gray. 17936, 25138.

Genistidium dumosum I.M. Johnst. 23005.

Gliricidia sepium (Jacq.) Kunth ex Walp. 11246.

Gliricidia sepium (Jacq.) Kunth ex Walp. 3139, 3331, 5713, 5869, 10230, 12581, 12735, 15741, 15922, 16234, 16288, 
16289.

Haematoxylum brasiletto H. Karst. 3193, 3388, 5850, 9975, 10048.

Harpalyce sousae Arroyo. 4209, 6164, 6995.

Harpalyce formosa Moc. \& Sessé ex DC. 4083, 6146.

Harpalyce sousae Arroyo. 3444, 4114, 5411, 5902, 6233.

Havardia pallens (Benth.) Britton \& Rose. 16827, 17950, 21422, 21578, 24159, 24442, 24451, 24619, 24979, 24980.

Helicotropis linearis (Kunth) A. Delgado. 4516, 4814.

Hoffmannseggia glauca (Ortega) Eifert. 16628, 17131, 19423, 24723, 28745, 28821.

Hoffmannseggia oxycarpa Benth. 18131.

Hoffmannseggia watsonii (Fisher) Rose. 18987, 18996, 25354.

Hybosema ehrenbergii (Schltdl.) Harms. 5928, 7983.

Indigofera constricta Rydb. 11606.

Indigofera cuernavacana Rose. 8090.

Indigofera densiflora M. Martens \& Galeotti. 1583, 8239, 11455, 17404bis.

Indigofera jaliscensis Rose. 6767.

Indigofera jamaicensis Spreng. 12079.

Indigofera lespedezioides Kunth. 6421, 8116, 10906, 16117.

Indigofera miniata Ortega. 1052, 1236, 6355, 6364, 6999, $9135,10477,11885,19380,20392,21198,21339,22016$, 22846, 24466, 24640, 27513, 27520.

Indigofera palmeri $\mathrm{S}$. Watson. 1217, 5856, 6112, 7829, 7850, 7884.

Indigofera suffruticosa Mill. 1178, 5452, 10193, 11055, 12236, 15178, 17628, 17946, 24285, 24887.

Indigofera thibaudiana DC. 1698, 2018, 7441, 12664, 12728, 15627.

Inga oerstediana Benth. ex Seem. 10746, 12942, 13718.

Inga schiedeana Steud. 456, 4182, 6171, 7617 (Type of Inga hintonii Sandwith), 7667, 8103, 8232, 8978, 11202.

Inga vera Willd. 3159, 3981, 5527, 5572, 5911, 6184, 6205, 6232, 6483, 7349, 8977, 9089, 9248, 9259, 9997, 10074.

Lathyrus parvifolius S. Watson. 17334, 18157, 18248, 18425, 18807, 18950, 20279, 22909, 23303, 25297.

Leptospron adenanthum (G. Mey.) A. Delgado. 1929, 10960, 11491, 11568, 11709, 14734, 15247.

Lespedeza texana Britton. 24668.

Leucaena greggii S. Watson. 17795.

Leucaena diversifolia (Schltdl.) Benth. 7993.

Leucaena esculenta (Moc. \& Sessé ex DC.) Benth. 1965, 2349, 5745, 7417, 13371.

Leucaena greggii S. Watson. 16695, 21733, $22044,24967$.

Leucaena leucocephala (Lam.) de Wit. 3119, 5138, 5408, 7287.

Leucaena macrophylla Benth. 2256, 5098, 5381, 7493, 8611, 10985, 12733, 14840, 16182.

Libidibia coriaria (Jacq.) Schltdl. 1839, 2221, 5851, 6121.

Lonchocarpus argyrotrichus Harms. 17234.

Lonchocarpus balsensis M. Sousa \& J.C. Soto. 7753.

Lonchocarpus constrictus Pittier. 5861, 5986, 6300, 10349, 10387.

Lonchocarpus epigaeus M. Sousa. 7741.

Lonchocarpus eriophyllus Benth. 1194, 6362, 8059.
Lonchocarpus guatemalensis Benth. 1473, 7763, 8179, 11729, 13745, 15818, 15827.

Lonchocarpus hintonii Sandwith. 726, 3772, 3934, 4121, 5571, 5936 (TYPUS), 5958, 5977, 6508, 8583, 9988, 10013, 14007.

Lonchocarpus lanceolatus Benth. 7288.

Lonchocarpus longipedicellatus Pittier. 10384, 10459.

Lonchocarpus minimiflorus Donn. Sm. 12546.

Lonchocarpus obovatus Benth. 3742, 5523, 5937, 5942, 5946, 6290, 6959, 7041.

Lonchocarpus parviflorus Benth. 6649, 7281, 8495, 10540, 16272.

Lonchocarpus punctatus Kunth. 12064.

Lonchocarpus rugosus Benth. 5891, 6325.

Lotus hintoniorum B.L. Turner. 22118 (TYPUS), 22962.

Lotus repens (G. Don) Sessé \& Moc. ex Standl. \& Steyerm. 805, 2442, 6255, 8773, 12920, 26169.

Lupinus stipulatus J. Agardh. 29814.

Lupinus aralloius C.P. Sm. 15592 (TYPUS).

Lupinus aschenbornii Schauer. 4626, 8290, 8340 (Type of Lupinus aschenbornii var. cervantesii C.P. Sm.), 15594, 15728.

Lupinus barkeri Lindl. 321, 5407, 6151, 6760, 7063, 7080, 8260, 8280, 8350, 8380, 9648, 9709, 9754, 9943, 10731, 11414.

Lupinus cacuminis Standl. 17002, 17038, 17326, 17883, 17978, 18820, 18873, 27531, 28342.

Lupinus campestris Schltdl. \& Cham. 11939.

Lupinus decaschistus C.P. Sm. 14786 (TYPUS).

Lupinus diehlii M.E. Jones. 13565, 15124.

Lupinus elegans Kunth. 8677, 8925, 8947, 9858, 11814, 12750, 12752, 12775, 13535 (Type of Lupinus elegans var. quercetorum C.P. Sm.), 13732, 13736, 14243 (Type of Lupinus metallactus C.P. Sm.), 15344.

Lupinus exaltatus Zucc. 5924, 7475, 8281, 8288, 8304, 8305, 8307, 8308, 8771, 8808, 8809, 8940, 8943, 8944, 8991, 9006, 9929, 10887bis, 11087, 12392, 12663 (Type of Lupinus fictomagnus C.P. Sm.), 12671, 12720 (Type of Lupinus monteportae C.P. Sm.), 12748, 12753, 12786, 12795, 13179 , 13347, 13466, 13509, 15010, 15087, 15089, 15273, 15564, 15599 (Type of Lupinus tancitaricus C.P. Sm.), 15654 (Type of Lupinus paraplesius C.P. Sm.), 15679, 15712 (Type of Lupinus dispar C.P. Sm.), 15724 (Type of Lupinus ferax C.P. Sm.).

Lupinus hintonii C.P. Sm. 781 (TYPUS), 8336, 9019, 11932.

Lupinus hintoniorum B.L. Turner. 19329, 22657, 22840, 22936 (TYPUS), 22967, 22972, 23456, 24014, 24036.

Lupinus jaimehintoniana B.L. Turner. 26105, 26160 (TYPUS), 26179, 26228, 26809.

Lupinus madrensis Seem. 9937 (Type of Lupinus squamecaulis C.P. Sm.), 10154, 12345, 12346, 12751, 12755, 13687.

Lupinus mexicanus Cerv. ex Lag. 8261, 8268, 8269, 8270, $8289,8302,8303,8318,8321,8960,8965,8994,9023$, 13362, 13450, 13493, 15403.

Lupinus montanus Kunth. 8244, 8317, 9031, 15593.

Lupinus octablomus C.P. Sm. 14521 (TYPUS).

Lupinus perbonus C.P. Sm. 15702 (TYPUS). 
Lupinus persistens Rose. 8243, 8315 (Type of Lupinus persistens var. praeclarus C.P. Sm.), 13237.

Lupinus platamodes C.P. Sm. 23201, 23925, 24594, 24910. Lupinus potosinus Rose. 17360, 17635, 17889, 18210, 18445, 18533, 18850, 21697, 24587.

Lupinus quercuum C.P. Sm. 8362 (TYPUS), 13285 (Type of Lupinus quercuum var. perflexolobius C.P. Sm.).

Lupinus reflexus Rose. 15646.

Lupinus rhodanthus C.P. Sm. 13117 (TYPUS).

Lupinus semiaequus C.P. Sm. 15596 (TYPUS).

Lupinus simulans Rose. 8937 (Type of Lupinus simulans var. abietum C.P. Sm.), 13414, 13505.

Lupinus splendens Rose. 8360.

Lupinus stipulatus J. Agardh. 17420, 23318, 24090.

Lupinus sylvaticus Hemsl. 8316.

Lupinus taurimortuus C.P. Sm. 11088 (TYPUS).

Lysiloma acapulcense (Kunth) Benth. 464, 705, 2784, 3760, 3838, 5904, 10006, 10132, 14250.

Lysiloma divaricatum (Jacq.) J.F. Macbr. 763, 933, 4269, 6125, 9162, 12303.

Lysiloma tergeminum Benth. 929, 4180, 5968, 6607, 10383.

Macroptilium gibbosifolium (Ortega) A. Delgado. 18000, 29721.

Macroptilium atropurpureum (Sessé \& Moc. ex DC.) Urb. 2269, 3583, 4745, 5007, 5107, 5415, 5575, 6045, 6671, 6734, 6866, 8505, 11775, 13207, 15315.

Macroptilium gibbosifolium (Ortega) A. Delgado. 1605, 12221, 13159, 13969, 24355.

Macroptilium gracile (Poepp. ex Benth.) Urb. 17735, 26481, 26497.

Macroptilium pedatum (Rose) Maréchal \& Baudet. 6602, 8512.

Machaerium isadelphum (E. Mey.) Amshoff. 3140, 3645.

Machaerium lunatum (L. f.) Ducke. 13777, 16283.

Machaerium salvadorense (Donn. Sm.) Rudd. 11726.

Marina diffusa (Moric.) Barneby. 2267, 11255.

Marina ghiesbreghtii Barneby. 11586 (TYPUS).

Marina gracilis Liebm. 1462, 1750, 6665, 9409, 13294, 15248.

Marina neglecta (B.L. Rob.) Barneby. 5846.

Marina nutans (Cav.) Barneby. 124, 2281, 2354, 2541, 2692 , 8658, 10997, 13376, 13428, 14769, 15576.

Marina spiciformis (Rose) Barneby. 1755, 1938, 13273, 15311.

Marina unifoliata (B.L. Rob. \& Greenm.) Barneby. 6035, 9719, 15299, 16158.

Mariosousa acatlensis (Benth.) Seigler \& Ebinger. 3747, 5417, 7749, 7755, 11248.

Mariosousa coulteri (Benth.) Seigler \& Ebinger. 4142, 17787, 17938, 24102, 24158, 24235, 24517.

Medicago lupulina L. 800, 19417, 19465.

Medicago polymorpha L. 3460.

Medicago sativa L. 5599.

Melilotus albus Desr. 17136, 18073, 19480, 19882.

Melilotus indicus (L.) All. 528, 2100, 3601, 12939, 13630, 17120 .

Microlobius foetidus (Jacq.) M. Sousa \& G. Andrade. 3811,
$5488,5883$.

Mimosa acapulcensis B.L. Rob. 26472.

Mimosa aculeaticarpa Ortega. 2853, 6814, 7337, 7693, 16606, 17537, 18401, 18669, 18740, 19385, 19466, 19611, 20951, 22169, 22943, 24067, 24309.

Mimosa adenantheroides (M. Martens \& Galeotti) Benth. 12709, 15023, 15090.

Mimosa affinis B.L. Rob. 1600, 1756, 4743, 5995.

Mimosa albida Humb. \& Bonpl. ex Willd. 1415, 1642, 4671, 6540, 9424, 10639, 10640, 10904, 11485, 11971, 14475.

Mimosa benthamii J.F. Macbr. 1237, 1644, 5410, 6405, 6831, 8424, 8890, 9427.

Mimosa borealis A. Gray. 18960, 20923.

Mimosa caerulea Rose. 1702, 7017, 8217, 8231, 8756, 8894, 17408bis.

Mimosa camporum Benth. 6628.

Mimosa diplotricha C. Wright ex Sauvalle. 2290, 6036, 9666.

Mimosa distachya Cav. 6298.

Mimosa egregia Sandwith. 6296, 6314 (TYPUS), 6907 (TYPUS), 7887, 8035, 8519.

Mimosa emoryana Benth. 16533.

Mimosa galeottii Benth. 898, 6248, 6256, 7185, 7197, 8427.

Mimosa guatemalensis (Hook. \& Arn.) Benth. 2481, 5102.

Mimosa lacerata Rose. 3773, 5791, 6865.

Mimosa lactiflua Delile ex Benth. 6546.

Mimosa leucaenoides Benth. 25241.

Mimosa malacophylla A. Gray. 17774, 21157, 24108, 24621.

Mimosa moreliensis (Britton) Bullock. 7446.

Mimosa pigra L. 10232.

Mimosa polyancistra Benth. 1088, 7835.

Mimosa polyantha Benth. 778, 3957, 4299, 5793, 5884, 6131, 6940, 7862, 7874, 8516, 8525 .

Mimosa potosina (Britton \& Rose) B.L. Turner. 20926, 22021, 24471.

Mimosa pudica L. 14629.

Mimosa quadrivalvis L. 12235.

Mimosa rhododactyla B.L. Rob. 12126, 13372.

Mimosa robusta R. Grether. 1300, 1475, 10628, 10836, 11001.

Mimosa somnians Humb. \& Bonpl. ex Willd. 8485, 11337. Mimosa spirocarpa Rose. 15324.

Mimosa tenuiflora (Willd.) Poir. 4381, 8068.

Mimosa tricephala Schltdl. \& Cham. 3802, 5839, 5885, 5984, 5985bis, 5987, 6864, 6915, 7055, 7838, 9058, 9156, 9161, 10510.

Mimosa zygophylla Benth. 16612, 22766, 22782, 25826, 27066, 28542.

Mucuna sloanei Fawc. \& Rendle. 8759, 10804, 10934.

Myrospermum frutescens Jacq. 7291.

Myroxylon balsamum (L.) Harms. 12296, 12298, 12540.

Neptunia oleracea Lour. 6652.

Neptunia plena (L.) Benth. 6495, 15205.

Nissolia fruticosa Jacq. 6709, 8163, 12682, 14486, 14546.

Nissolia hintonii Sandwith. 2213, 2334 (TYPUS), 2520, 2922, 3335, 5075, 5603, 7086, 7189. 
Nissolia laxior (B.L. Rob.) Rose. 771, 4028, 13886. Nissolia leiogyne Sandwith. 6291 (TYPUS), 6616, 7861. Nissolia microptera Poir. 1197, 4319, 6280, 6692, 9142. Nissolia platycalyx S. Watson. 16532, 19614, 24458. Orbexilum melanocarpum (Benth.) Rydb. 18539, 18684. Oxyrhynchus populneus (Piper) Norvell ex A. Delgado \& E. Estrada. 21235.

Pachyrhizus erosus (L.) Urb. 1205, 4424, 9367, 9540, 11486 , 11776, 13152, 14478, 14673.

Painteria elachistophylla (S. Watson) Britton \& Rose. 21832, 27854.

Painteria leptophylla (DC.) Britton \& Rose. 19239, 19378, 19379, 20813, 21394, 22175.

Parkinsonia aculeata L. 3793, 5476, 5642, 28313.

Parkinsonia praecox (Ruiz \& Pav. ex Hook.) J. Hawkins. 3794, 5455, 6040, 9968.

Parkinsonia texana (A. Gray) S. Watson. 16651, 24176.

Peteria glandulosa (A. Gray) Rydb. 23109, 23703.

Peteria scoparia A. Gray. 29295.

Phaseolus coccineus L. 18026.

Phaseolus neglectus F.J. Herm. 17107.

Phaseolus pluriflorus Maréchal, Mascherpa \& Stainier. 18025.

Phaseolus acutifolius A. Gray. 25108.

Phaseolus anisotrichos Schltdl. 2266, 2287, 6642, 9382, 9485, 9567, 10601, 10827, 10947, 13163, 15631, 17697, 17797, 21391, 22564.

Phaseolus coccineus L. 623, 2006, 2310, 8245, 8279, 9755, 12261, 13276, 15509, 22235, 23251, 23477, 24602, 24691, 24898, 26054, 26242, 26366.

Phaseolus filiformis Benth. 15781.

Phaseolus hintonii A. Delgado. 6728 (TYPUS).

Phaseolus lunatus L. 3100, 3121, 5383, 5439, 11742 , 11771.

Phaseolus microcarpus Mart. 8584.

Phaseolus neglectus F.J. Herm. 17106, 19177, 21258, 23816, 24657, 24908, 25146.

Phaseolus nelsonii Maréchal, Mascherpa \& Stainier. 4714, 4799, 12191.

Phaseolus pauciflorus Sessé \& Moc. ex G. Don. 1555, 1588, 4653, 4708, 15137.

Phaseolus pedicellatus Benth. 2375, 22479, 23241, 23300, 23304.

Phaseolus polymorphus S. Watson. 17245, 23421, 23424.

Phaseolus vulgaris L. 5605, 11551, 11567, 11684.

Piptadenia flava (Spreng. ex DC.) Benth. 4506, 5468, 6635, 6705, 9157, 26485.

Piptadenia obliqua (Pers.) J.F. Macbr. 9063, 9991, 10321, 14336, 16102.

Piscidia carthagenensis Jacq. 3578, 5626, 5803, 7524, 9995, 10008, 11802, 14532, 14869.

Piscidia grandifolia (Donn. Sm.) I.M. Johnst. 1193 (Type of Piscidia grandifolia var. glabrescens Sandwith), 5419, 8079, 8603, 10518, 13057.

Pithecellobium dulce (Roxb.) Benth. 453, 3002, 3192, 3931, 5430, 5524, 5812, 5966, 7591, 12970.

Pithecellobium lanceolatum (Humb. \& Bonpl. ex Willd.) Benth. 5614, 5819, 13904.
Pithecellobium unguis-cati (L.) Benth. 5865, 7756, 8515, 10871.

Platymiscium jejunum Klitg. 14001 (TYPUS).

Platymiscium lasiocarpum Sandwith. 3174 (TYPUS), 3389, 5396, 7320, 7406, 7503, 7507, 7525, 7532, 7552, 7922, 12952, 12989, 13778.

Poeppigia procera C. Presl. 4556, 5703, 6106, 8042, 9173. Poiretia punctata (Willd.) Desv. 5142, 5361, 6751.

Pomaria canescens (Fisher) B.B. Simpson. 22877, 25892.

Pomaria melanosticta S. Schauer. 23368.

Prosopis glandulosa Torr. 24071.

Prosopis juliflora (Sw.) DC. 3803, 5471, 7813, 10886, 16592, 16613, 19664, 22174, 22791, 24970, 27560.

Prosopis reptans Benth. 28798.

Pterocarpus orbiculatus DC. 3607, 3965, 5699, 5881, 9985, 9987, 11801.

Ramirezella lozanii (Rose) Piper. 12917, 15782.

Ramirezella micrantha A. Delgado \& Ochoterena-Booth. 14658.

Ramirezella strobilophora (B.L. Rob.) Rose. 1989, 3441, 5574, 7415, 7782, 7897, 7935, 9350, 10927, 11634, 12479, 16138.

Rhynchosia discolor M. Martens \& Galeotti. 3058, 3197, 7342, 8659, 15777, 15778.

Rhynchosia edulis Griseb. 2258, 7336, 10569.

Rhynchosia longeracemosa M. Martens \& Galeotti. 17692, 24836, 24886.

Rhynchosia minima (L.) DC. 2230, 3163, 3786, 5434, 10945, 26463.

Rhynchosia potosina Brandegee. 1182.

Rhynchosia precatoria (Humb. \& Bonpl. ex Willd.) DC. 2280, 3074, 5399, 13587, 14036.

Rhynchosia senna Gillies ex Hook. \& Arn. 25454.

Robinia pseudoacacia L. 16749.

Securigera varia (L.) P. Lassen. 24138.

Senegalia berlandieri (Benth.) Britton \& Rose. 16576, 16773, 17518, 19273, 19927, 21078, 21534, 21731, 21833, 24430.

Senegalia crassifolia (A. Gray) Britton \& Rose. 23356, 23547, 28466, 28532.

Senegalia greggii (A. Gray) Britton \& Rose. 16626.

Senegalia macilenta (Rose) Britton \& Rose. 3355, 5507, 5748, 7269, 11745.

Senegalia polyphylla (DC.) Britton \& Rose. 5737, 10785, 12548, 16110.

Senegalia riparia (Kunth) Britton. 15212.

Senegalia roemeriana (Scheele) Britton \& Rose. 21230, 21474, 22170, 24178.

Senegalia wrightii (Benth.) Britton \& Rose. 16786, 25745. Senna pilifera (Vogel) H.S. Irwin \& Barneby. 461.

Senna alata (L.) Roxb. 5660, 6904, 10887, 10956, 11764.

Senna andrieuxii (Benth.) H.S. Irwin \& Barneby. 5894.

Senna argentea (Kunth) H.S. Irwin \& Barneby. 6961.

Senna atomaria (L.) H.S. Irwin \& Barneby. 10237, 10262, 10876, 11782, 12155, 13613, 13628, 13751.

Senna centranthera H.S. Irwin \& Barneby. 12487, 14925, 15995.

Senna crotalarioides (Kunth) H.S. Irwin \& Barneby. 16696, 17397, 18965, 23100. 
Senna demissa (Rose) H.S. Irwin \& Barneby. 29181. Senna durangensis (Rose) H.S. Irwin \& Barneby. 28845. Senna foetidissima (G. Don) H.S. Irwin \& Barneby. 6530 (Type of Cassia stenosepala L.B. Sm. \& B.G. Schub.), 11876, 12491, 13582.

Senna fruticosa (Mill.) H.S. Irwin \& Barneby. 1517, 4330, 7053, 10983, 11479, 11796, 12645.

Senna galegifolia (L.) Barneby \& Lourteig. 14153.

Senna hirsuta (L.) H.S. Irwin \& Barneby. 1809, 9585, 10842, 11430, 11616, 12199.

Senna holwayana (Rose) H.S. Irwin \& Barneby. 11297.

Senna lindheimeriana (Scheele) H.S. Irwin \& Barneby. 16875, 17625, 19893, 20066, 21181, 22189, 23787.

Senna mensicola (H.S. Irwin \& Barneby) H.S. Irwin \& Barneby. 19127, 19592, 27205.

Senna mexicana (Jacq.) H.S. Irwin \& Barneby. 1125.

Senna mollissima (Willd.) H.S. Irwin \& Barneby. 11740.

Senna multifoliolata (Paul G. Wilson) H.S. Irwin \& Barneby. 9945 (Type of Senna multifoliolata var. metaxi H.S. Irwin \& Barneby), 10177, 12352 (TYPUS), 12353, 14785.

Senna multiglandulosa (Jacq.) H.S. Irwin \& Barneby. 493, 2877, 4090, 6216, 13546.

Senna nicaraguensis (Benth.) H.S. Irwin \& Barneby. 6758, 11425.

Senna obtusifolia (L.) H.S. Irwin \& Barneby. 448, 6021, 11439, 11617.

Senna occidentalis (L.) Link. 4735, 5677, 9359, 11370, 11600, 15176, 17538, 22884, 24569.

Senna pallida (Vahl) H.S. Irwin \& Barneby. 355, 2972, 5582 (Type of Cassia trichocraspedon Sandwith), 5742, 6144 (Type of Cassia tortuosa L.B. Sm. \& B.G. Schub.), 8917, 9034, 10928, 11264, 11512 (Type of Senna pallida var. triquetripes H.S. Irwin \& Barneby), 11539, 12618, 12688, 13634, 14915, 15847, 16012.

Senna pendula (Humb. \& Bonpl. ex Willd.) H.S. Irwin \& Barneby. 5889, 12980, 14119, 15879.

Senna pilifera (Vogel) H.S. Irwin \& Barneby. 1791, 4816, 4841, 7100, 11559, 11746.

Senna pilosior (J.F. Macbr.) H.S. Irwin \& Barneby. 23577, 24749.

Senna pumilio (A. Gray) H.S. Irwin \& Barneby. 22997.

Senna quinquangulata (Rich.) H.S. Irwin \& Barneby. 12547.

Senna racemosa (Mill.) H.S. Irwin \& Barneby. 3154, 12640 (Type of Senna racemosa var. coalcomanica H.S. Irwin \& Barneby), 16105.

Senna septemtrionalis (Viv.) H.S. Irwin \& Barneby. 662, 8278, 15436.

Senna skinneri (Benth.) H.S. Irwin \& Barneby. 475, 1447, 6114, 6622, 9177, 12112, 13008.

Senna uniflora (Mill.) H.S. Irwin \& Barneby. 1449, 5375, 5620, 9360, 11422, 11750.

Senna wislizeni (A. Gray) H.S. Irwin \& Barneby. 6305, 6612, 16565 (Type of Cassia monozyx H.S. Irwin \& Barneby), 17810, 17906, 19470, 21118, 21825, 22265, 22323, 27368, 28652.

Sesbania herbacea (Mill.) McVaugh. 1985, 4729, 5618.

Sigmoidotropis speciosa (Kunth) A. Delgado. 11628,
11738.

Sophora nuttalliana B.L. Turner. 18414, 25310.

Sophora tomentosa (B.L. Turner) B.L. Turner. 24053 (TYPUS).

Sphinctospermum constrictum (S. Watson) Rose. 1470, 4732, 5989, 6474, 6507, 6627.

Sphinga acatlensis (Benth.) Barneby \& J.W. Grimes. 725, 928, 4140, 5658, 9989.

Stenolobium brachycarpum Benth. 2247, 5156, 10905.

Stylosanthes guianensis (Aubl.) Sw. 1931, 4767, 6985, 11456.

Stylosanthes humilis Kunth. 15289.

Stylosanthes mexicana Taub. 21471.

Swartzia simplex (Sw.) Spreng. 10222, 10632, 14355, 15117, 15894, 15951, 16230.

Tamarindus indica L. 688, 927, 3576, 5971.

Tara cacalaco (Bonpl.) Molinari \& Sánchez Och. 12925, 13366, 16199.

Tara vesicaria (L.) Molinari, Sánchez Och. \& Mayta. 10865, 10992.

Tephrosia cinerea (L.) Pers. 1939, 6603, 26477.

Tephrosia conzattii (Rydb.) Standl. 5273, 6998, 10485.

Tephrosia cuernavacana (Rose) J.F. Macbr. 1297, 15051.

Tephrosia diversifolia (Rose) J.F. Macbr. 12719.

Tephrosia macrantha B.L. Rob. \& Greenm. ex Pringle. 5099, 5172, 11558, 14999.

Tephrosia major Micheli. 14998.

Tephrosia mexicana C.E. Wood. 2698 (TYPUS).

Tephrosia multifolia Rose. 16180.

Tephrosia nicaraguensis Oerst. 1278.

Tephrosia pogonocalyx C.E. Wood. 3101 (TYPUS).

Tephrosia potosina Brandegee. 25317, 27521.

Tephrosia purpurea (L.) Pers. 5180, 6645, 16116.

Tephrosia rhodantha Brandegee. 1921, 11403, 11641.

Tephrosia sinapou (Buc'hoz) A. Chev. 4683, 8114.

Tephrosia vernicosa C.E. Wood. 9950 (TYPUS).

Tephrosia vicioides Schltdl. 5096.

Teramnus uncinatus (L.) Sw. 3830.

Trifolium amabile Kunth. 1311, 1374, 2091, 4630, 4905, 12419, 13108, 15266, 15559, 22213, 22923, 23096, 23422.

Trifolium dubium Sibth. 18792.

Trifolium wormskioldii Lehm. 796, 1390, 13114, 16666.

Vachellia campechiana (Mill.) Seigler \& Ebinger. 1173, 4124, 5967, 5988, 6284, 6286, 6979, 7418, 7764, 7814, 7868, 9140, 9420, 10021, 10265, 10388, 11487.

Vachellia constricta (Benth.) Seigler \& Ebinger. 10333, 16501, 16585, 16741, 21890, 22604, 24742, 27437, 28366. Vachellia farnesiana (L.) Wight \& Arn. 376, 1416, 3369, 5615, 13100, 17751, 20011, 21107, 22168, 25300.

Vachellia glandulifera (S. Watson) Seigler \& Ebinger. 19471, 25761, 27842.

Vachellia hindsii (Benth.) Seigler \& Ebinger. 14120.

Vachellia pennatula (Schltdl. \& Cham.) Seigler \& Ebinger. 601, 3426, 5934, 6022, 6973, 9986, 10040, 13642.

Vachellia rigidula (Benth.) Seigler \& Ebinger. 5698, 7274, 17731, 21539, 21774, 21819, 21849, 22171, 22605, 24982, 25222.

Vicia hirsuta (L.) A. Gray. 4895. 
Vicia humilis Kunth. 4912, 6840, 8897, 17005, 18162, 18208, 18314, 19117, 20985, 22233, 24021, 24049, 24811.

Vicia ludoviciana Nutt. ex Torrr. \& A. Gray. 18435, 18527. Vicia pulchella Kunth. 2812, 8284, 13452, 17041, 18804, 19150, 19400, 20271, 23306, 26025, 26715.

Vicia sativa L. 396, 2456.

Vigna luteola (Jacq.) Benth. 5541, 5646, 5654, 6002. Vigna umbellata (Thunb.) Ohwi \& Ohashi. 5822.

Zapoteca formosa (Kunth) H.M. Hern. 6466, 6878, 7094, 9191, 10894, 11626, 12558, 13053, 14335, 14605, 15131.

Zapoteca portoricensis (Jacq.) H.M. Hern. 2019.

Zapoteca tetragona (Willd.) H.M. Hern. 2207, 7101, 10847, 11059, 11577, 11780, 12274, 12331, 14707, 15278.

Zornia thymifolia Kunth. 17999.

Zornia diphylla (L.) Pers. 1655, 1665, 1803, 1883, 4499, 4756, 4868, 6382, 9179, 9571, 10767, 11427, 11640, 12244, 15292.

Zornia gemella (Willd.) Vogel. 25050.

Zygia conzattii (Standl.) Britton \& Rose. 10782.

\section{Family Fagaceae}

Quercus crassipes Bonpl. 29815, 29816, 29817.

Quercus deserticola Trel. 29728, 29790.

Quercus frutex Trel. 29804, 29827.

Quercus galeanensis C.H. Müll. 27174, 27177, 27978, 28077.

Quercus greggii (A. DC.) Trel. 19365.

Quercus laeta Liebm. 29810, 29811.

Quercus magnoliifolia Née. 11913.

Quercus mexicana Bonpl. 29044, 29045.

Quercus obtusata Bonpl. 17989.

Quercus polymorpha Schltdl. \& Cham. 16914.

Quercus pringlei Seemen. 28063.

Quercus pungens Liebm. 19007.

Quercus rugosa Née. 29865.

Quercus rysophylla Weath. 16916.

Quercus saltillensis Trel. 25530.

Quercus sideroxyla Bonpl. 25175, 27387.

Quercus acutifolia Née. 6087, 6140, 6224, 6238, 6330, 6384, 6387, 6389, 6399, 6400, 6516, 6589, 6591, 7570, 9216, 10374, 10729, 10737, 12148, 12149, 12151, 12152, 12153, 13195, 13730, 14411, 14421, 14850, 15701.

Quercus affinis Scheidw. 17267, 18338.

Quercus aristata Hook. \& Arn. 6514, 14203, 14405.

Quercus calophylla Schltdl. \& Cham. 6147, 6169, 6358, 6361, 6515, 6761, 6763, 6801, 9569, 10673, 10722, 11912, 12341, 13672, 13696, 14953, 15700, 17435bis.

Quercus canbyi Trel. 17286, 17717, 17720, 17811, 18395, 18397, 20179, 21195, 22989, 23409, 24459, 24551, 25312, 25375, 25924, 25939, 28152.

Quercus castanea Née. 5780, 6210, 6217, 6398, 6593, 6737, 6738, 6804, 7188, 7193, 9225, 9226, 9316, 10736, 11890, $13344,13345,13437,13468,13545,13559,13560,13685$, $15597,15713$.

Quercus coccolobifolia Trel. 22675, 22676, 24142.

Quercus conzattii Trel. 26781, 26821, 26823.

Quercus crassifolia Bonpl. 3283, 3288, 3451, 5920, 5935, 6083, 6243, 6401, 6402, 6403, 6572, 6573, 6574, 6586, 7196,
9743, 13675, 13682, 13697, 15026, 23931, 23935.

Quercus crassipes Bonpl. 6084, 6094, 6583, 13523, 13547, 13681, 15348, 17435.

Quercus diversifolia Née. 19255, 19256, 22558, 25227.

Quercus durifolia Seemen. 17269, 18848, 21325, 21327, 21635, 23447, 24924, 28052.

Quercus eduardi Trel. 19006, 19081, 19219, 21356, 22296, 24377, 25292, 25293.

Quercus elliptica Née. 3102, 5346, 6060, 6062 (Type of Quercus peradifolia E.F. Warb.), 7068, 7363.

Quercus fusiformis Small. 17955, 21223, 21465, 28137, 28139.

Quercus galeanensis C.H. Müll. 18249, 18623, 18670, 20192, 27972, 27974, 27994, 28019, 28064, 28065.

Quercus gambelii Nutt. 6409.

Quercus glaucoides M. Martens \& Galeotti. 4503, 6195, 6200, 6363, 6385, 6561, 9051, 9198, 13099, 14393, 17666, 24219, 25248.

Quercus greggii (A. DC.) Trel. 17433, 17442, 17453, 17454, 17461, 17887, 18205, 18349, 18350, 18356, 18359, 18367, 18601, 18953, 19046, 19109, 20146, 20977, 22838, 23943, 23950, 24379, 27538, 27968, 28000.

Quercus hintonii E.F. Warb. 2835, 3096, 5779, 5913, 6072, 6073, 6074, 6077, 6148, 6199, 6225, 6237, 6327, 6349, 6350, 6359 (TYPUS), 6376, 6392, 6568 (Type of Quercus apiophylla E.F. Warb.), 6569, 6570, 6576 (Type of Quercus ochroesthes E.F. Warb.), 6577 (Type of Quercus sagata E.F. Warb.), 6588, 6810.

Quercus hintoniorum Nixon \& C.H. Müll. 18355, 18360, 18428, 18849, 19364, 20199, 20978, 21097, 27539, 28214. Quercus hypoxantha Trel. 17265, 17266, 17279, 18365, 18582, 19298, 19372, 19457, 19458, 19460, 19461, 20143, 20145, 20233, 20966.

Quercus intricata Trel. 16740, 16763, 18087, 18730, 20158, 21075, 24489, 25923, 27855, 27896.

Quercus invaginata Trel. 18393, 21145, 23608.

Quercus laceyi Small. 25922, 28202, $28208,28213$.

Quercus laeta Liebm. 732, 5933, 6218, 6331, 6332, 6564, 6565, 16917, 17472, 18408, 19136, 21205, 21326, 21409, 21473, 22381, 22477, 23407, 23446, 23670, 23905, 23919, 23939, 24040, 24293, 24436, 24552, 24588, 24692, 24694, 24889, 24940, 25109, 27486, 27492, 28153.

Quercus lancifolia Schltdl. \& Cham. 14520.

Quercus laurina Bonpl. 828, 3263, 3296, 3693, 5782, 6582, 7104, 11954, 13028, 13703, 15691, 17455, 19459, 22647, $22673,22835,22844,24662,25123,25170,25182,25207$, 25304, 25316.

Quercus magnoliifolia Née. 642, 643, 3504, 4038, 5342, 5778, 6058, 6064, 6071, 6088, 6165, 6172, 6198, 6204, 6220, $6235,6242,6247,6252,6259,6326,6329,6360$ (Type of Quercus platyphylla E.F. Warb.), 6386, 6388, 6390, 6391, 7443, 9235, 9241, 9313, 10024, 10454, 10515, 10613, 11948, 13688, 13724.

Quercus mexicana Bonpl. 17431, 17436, 17462, 18671, 21304, 22476, 23416, 23448, 23936, 24039, 24558, 24926, 25097, 25291, 25294, 27484, 27485, 28215.

Quercus microphylla Née. 18116, 18391, 18680, 18682, 19297, 19386, 20727, 21799, 25448, 27300, 27537, 27638, 
27847, 27969, 27990, 28041, 28480

Quercus miquihuanensis Nixon \& C.H. Müll. 18947, 19027, 19296.

Quercus obtusata Bonpl. 3475, 3481, 3548, 3836, 5901, 6549 (Type of Quercus atriglans E.F. Warb.), 6585, 6592, 6773, 6805, 6993, 6994, 7194, 7198, 9217, 9227, 9233, $9299,9317,9572,9647,9655,11377,13694,15229,15490$, $15628,15723$.

Quercus opaca Trel. 20041, 20178, 23512, 24462, 24480, 24546, 24961, 25178.

Quercus peduncularis Née. 6378 (Type of Quercus dolichopus E.F. Warb.), 6555, 8422, 9297, 9312, 9314, 9315, 10023, 10079, 10084, 10213, 12245, 13029, 13097, 13719, 13747, 14044.

Quercus polymorpha Schltdl. \& Cham. 17481, 17490, 17490bis, 17512, 17714, 17715, 17716, 17800, 18091, 20180, 20222, 21206, 21224, 21413, 21415, 21536, 24166, 24544, 24548, 24635, 24983, 25128, 25245, 25254.

Quercus pringlei Seemen. 16694, 16751, 17459, 17467, $18078,18117,18189,18237,18348,18697,19162,19352$, 19682, 20112, 20480, 20716, 20862, 21734, 23750, 24062, 24479, 25229, 27006, 27007, 27305.

Quercus rugosa Née. 829, 6085, 6806, 6807, 7108, 13479, 13503, 13704, 14792, 15449, 15470.

Quercus rysophylla Weath. 22624, 22992, 23295, 23826, 24101, 24526, 25046, 25047, 25250.

Quercus salicifolia Née. 9901, 10214, 10292, 13748, 14363.

Quercus saltillensis Trel. 16734, 17578, 18374, 24927, 24928, 24934, 25439, 27976.

Quercus scytophylla Liebm. 305, 3242, 3244, 6089, 6091, 6136, 6156, 6203, 6239, 6246, 6348, 7379, 9291, 13726.

Quercus sideroxyla Bonpl. 17430, 17432, 17434, 17441, 17456, 17457, 20176, 22979, 23408, 24589, 24670, 24695, 24860, 25071, 25168, 28050.

Quercus splendens Née. 6556.

Quercus toumeyi Sarg. 17268.

Quercus urbanii Trel. 3224, 3423, 4079, 6149, 6241.

Quercus vaseyana Buckley. 25795, 27503.

Quercus xalapensis Bonpl. 24818, 24904, 25205, 25206, 25271.

\section{Family Fouquieriaceae}

Fouquieria formosa Kunth. 2608, 7070, 7536.

Fouquieria shrevei I.M. Johnst. 28360.

Fouquieria splendens Engelm. 28493.

\section{Family Frankeniaceae}

Frankenia margaritae Medrano. 29878.

Frankenia gypsophila I.M. Johnst. 18224, 19535, 19563, 19643, 23028, 27546.

Frankenia margaritae Medrano. 28590, 28675.

\section{Family Garryaceae}

Garrya glaberrima Wangerin. 17500, 18687, 18773.

Garrya laurifolia Hartw. ex Benth. 5922, 6852, 17281, 20139, 20204, 21358, 22426, 22642, 24685, 24816, 24850, 24902, 25198, 25204, 27936, 28067, 28076, 28089.
Garrya longifolia Rose. 342, 553, 2385, 3201, 3605, 3666, 8948, 15641, 15698.

Garrya ovata Benth. 17466, 17886, 18735, 22405, 28091, 28097.

\section{Family Gentianaceae}

Eustoma barkleyi Shinners. 28780.

Eustoma exaltatum (L.) Salisb. ex G. Don. 5643, 5830, 5969, 17830, 18273, 20327, 29270.

Geniostemon gypsophilum B.L. Turner. 22354 (TYPUS), 25524, 27333, 27377, 27967, 28026.

Gentiana bicuspidata (G. Don) Briq. 3256.

Gentiana calyculata Lex. 459, 3707, 3810, 6819, 7663, 7837, 9033, 11201, 13690, 13781.

Gentiana hintoniorum B.L. Turner. 23961 (TYPUS).

Gentiana ovatiloba Kusn. 26081, 26751.

Gentiana spathacea Kunth. 524, 8816, 8903, 8973, 13540, 13551, 15749, 23940.

Gentianella amarella (L.) Börner. 1317, 2117, 8313, 8320, 8330, 17244.

Gyrandra brachycalyx (Standl. \& L.O. Williams) G. Mans. 7203, 8386, 26514.

Gyrandra chironioides Griseb. 17570, 18279, 21882, 22042, 28020, 28079.

Gyrandra tenuifolia (M. Martens \& Galeotti) G. Mans. 9917.

Halenia brevicornis (Kunth) G. Don. 2316, 2905, 3045 , $5257,5354,8311,8345,9886,11695,13412,13422,13424$, 13552, 14463, 15378, 15558, 17427bis, 18342.

Halenia conzattii Greenm. 26037, 26687.

Halenia crassiuscula B.L. Rob. \& Seaton. 787.

Halenia hintonii Bullock. 8273 (TYPUS).

Halenia plantaginea (Kunth) G. Don. 786, 1034, 4623, 13013, 13175, 15648, 22223, 23426, 26658, 26684.

Halenia recurva (J.E. Sm.) C.K. Allen. 20450.

Irlbachia alata (Aubl.) Maas. 14669.

Sabatia stellaris Pursh. 17901.

Schultesia guianensis (Aubl.) Malme. 4774, 5035, 11611, 14656, 16151.

Swertia radiata (Kellogg) Kuntze. 17299, 17586.

Zeltnera calycosa (Buckley) G. Mans. 17857, 18607, 20909, 21737, 22080, 22313, 25820, 27072, 28255.

Zeltnera quitensis (Kunth) G. Mans. 2773, 2855, 3150, 3704, 8660, 12867.

Zeltnera setacea (Benth.) G. Mans. 5170.

Zeltnera stricta (Schiede) Druce. 397, 3290, 3323, 5347, 6050 .

\section{Family Geraniaceae}

Erodium cicutarium (L.) L'Hér. ex Aiton. 18112.

Erodium cicutarium (L.) L'Hér. ex Aiton. 878, 4128, 8354, 8403, 17764, 18545, 19362, 19484, 20105, 24022, 27578.

Erodium moschatum (L.) L'Hér. ex Aiton. 4916, 5329, 26190.

Geranium hernandesii DC. 18031.

Geranium seemannii Peyr. 18015.

Geranium alpicola Loes. 26533.

Geranium caespitosum James. 25419. 
Geranium clarum Small. 14759.

Geranium crenatifolium H.E. Moore. 17014, 17031, 17056, 17114, 17335, 17873, 18821, 20283, 20984, 20990, 21000, 22842, 22956, 24385.

Geranium cruceroense Knuth. 4188, 4617 (TYPUS), 7971. Geranium hernandesii DC. 501, 3567, 6250, 7220, 7470, 8913, 8927, 9853, 13119, 13417, 13986, 15932.

Geranium hintonii H.E. Moore. 14787 (TYPUS).

Geranium hystricinum H.E. Moore. 14972 (TYPUS).

Geranium latum Small. 1887 (Type of Geranium pinetorum R. Knuth).

Geranium lilacinum R. Knuth. 7199, 8945 (TYPUS), 17491bis.

Geranium mexicanum Kunth. 483, 1327 (Type of Geranium temascaltepecense R. Knuth), 1723, 2677, 2891, 4533, 4533bis, 4621, 4915, 5428, 6393, 7019, 7996, 15265, 15557.

Geranium miahuatlanum B.L. Turner. 26552, 26744 (TYPUS), 26759.

Geranium monanthum Small. 26389, 26564, 26666, 26843. Geranium potentillifolium DC. 782, 3988, 6079, 11931.

Geranium potosinum H.E. Moore. 17013, 17022.

Geranium richardsonii Fisch. \& Traut. 8271, 8339.

Geranium seemannii Peyr. 6811, 16815, 17467bis, 17874, 19550, 20453, 21320, 21622, 22206, 26038, 26114bis, 26287, 26340, 29173.

Geranium tenue Hanks. 20993.

Geranium unguiculatum H.E. Moore. 14906 (TYPUS).

\section{Family Gesneriaceae}

Achimenes antirrhina (DC.) C.V. Morton. 1775, 4654, 4761, 8263, 9422, 10660, 10671, 12161, 14929, 15993, 17401bis. Achimenes bella C.V. Morton. 4512 (TYPUS), 8219, 9325, 10662, 12122, 12186, 15080.

Achimenes brevifolia C.V. Morton. 10766 (TYPUS).

Achimenes dulcis C.V. Morton. 8207 (TYPUS), 15064, 15116, 15428.

Achimenes erecta (Lam.) H.P. Fuchs. 1836, 4806, 8191, 8419, 9444, 10674, 10676, 10677.

Achimenes fimbriata Rose ex C.V. Morton. 4728, 4753, 8214, 9448, 11334.

Achimenes flava C.V. Morton. 1776, 4485 (TYPUS), 8421, 8443, 10583 (Type of Achimenes flava var. puberula C.V. Morton), 12173, 15120, 15187, 15429, 15793.

Achimenes glabrata (Zucc.) Fritsch. 1978, 2133, 4548, 4807, 4853, 8230, 9335, 9456, 10655, 11349, 12162.

Achimenes grandiflora (Schiede) DC. 8209, 9252, 10675, 11406, 15046.

Achimenes heterophylla (M. Martens) DC. 1422, 1579, 4657, 17403bis.

Achimenes longiflora DC. 9899, 10679, 11341, 12131, 12163, 14627, 15141, 15997.

Achimenes obscura C.V. Morton. 4705 (TYPUS), 8165, 8180, 9361, 11352, 13225, 14563.

Achimenes patens Benth. 1366, 1424, 4656, 8161, 9326, 12036, 12060, 13133, 14474, 15218.

Drymonia serrulata (Jacq.) Mart. 13837.

Eucodonia andrieuxii (DC.) Wiehler. 10661.
Kohleria tigridia (Ohlend.) Roalson \& Boggan. 10582, 10616.

Moussonia deppeana (Schltdl. \& Cham.) Hanst. 11023, 11696, 14031, 14668, 14977.

Moussonia elegans Decne. 3099, 5339, 7623, 15582, 17082.

Niphaea mexicana C.V. Morton. 1277, 4544 (TYPUS), 8203.

Phinaea multiflora C.V. Morton. 10626 (TYPUS), 10761, 14621, 15115, 15945, 15968.

Solenophora insignis (M. Martens \& Galeotti) Hanst. 14747.

\section{Family Grossulariaceae}

Ribes ciliatum Humb. \& Bonpl. ex Roem. \& Schult. 486, 3258, 3694, 5770, 7448, 8819 .

Ribes microphyllum Kunth. 604, 3685, 18819, 22427.

Ribes neglectum Rose. 9002, 17044, 17336, 18810.

Family Hamamelidaceae

Matudaea trinervia Lundell. 3090, 6163, 7381 (Type of Matudaea hirsuta Lundell).

\section{Family Heliotropaceae}

Euploca confertifolia (Torr.) Feuillet \& Halse. 20373.

Euploca fruticosa (L.) J.I.M. Melo \& Semir. 1218, 6042, 6640, 8181, 9079, 9159, 10513, 12054, 12061, 12078, 13089, 17951, 24452, 24478.

Euploca greggii (Torr.) Halse \& Feuillet. 23381, 24761, 28432, 28820.

Euploca procumbens (Mill.) Diane \& Hilger. 701, 735, 773, 4364, 5624, 7811, 7820, 10483, 13194, 13963, 14345, 14538, 19976.

Euploca torreyi (I.M. Johnst.) Halse \& Feuillet. 16544, 17652, 17831, 19941, 20003, 20376, 20379, 20821, 21773, 22336, 22787, 23008, 24069, 24270, 28259, 28382, 28516, 28863, 29014.

Euploca confertifolia (Torr.) Feuillet \& Halse. 19972, 23018, 23741, 27647, 29416.

Heliotropium angiospermum Murray. 5673, 7856, 11981, 12997, 13138, 13637, 13649, 17530, 19949, 20046, 20099, 20356, 21424, 22876, 23617, 24272, 24444, 25517, 25660, 27749.

Heliotropium calcicola Fernald. 22186, 25001.

Heliotropium confertifolium (Torr.) A. Gray. 17527.

Heliotropium cremnogenum I.M. Johnst. 8514 (TYPUS).

Heliotropium curassavicum L. 1166, 3614, 7915, 16724, 17813, 19436, 26479, 27607, 29299.

Heliotropium fallax I.M. Johnst. 2599, 3631 (Type of Heliotropium fallax var. hintonii I.M. Johnst.).

Heliotropium indicum L. 774, 5678, 9155, 12990, 13037, 14344.

Heliotropium lagoense (Warm.) Gürke. 736.

Heliotropium limbatum Benth. 946, 4439, 7714, 9197, 10364, 12160, 13056, 13973.

Heliotropium michoacanum I.M. Johnst. 15069 (TYPUS).

Heliotropium molle (Torr.) I.M. Johnst. 24757, 24767, 28431. 
Heliotropium rufipilum (Benth.) I.M. Johnst. 11198, 11523, 12516, 12610, 12672.

Tournefortia acutiflora M. Martens \& Galeotti. 2055, 2802, 3553, 5285, 12732, 15013, 15504, 17240.

Tournefortia calycina Benth. 1994, 2182, 10996.

Tournefortia hartwegiana Steud. 12511, 12526, 12642, 12827, 13629.

Tournefortia hirsutissima L. 14156, 15114, 15804.

Tournefortia mutabilis Vent. 2477, 5116, 5482, 11582, $12446,12536,12695,12811,13488,13490,14817,15828$, 16243 .

Tournefortia volubilis L. 3559, 7032, 13636.

\section{Family Hernandiaceae}

Gyrocarpus americanus Jacq. 3161, 10987, 15890, 16187.

\section{Family Hydrangeaceae}

Fendlera linearis Rehder. 16566, 21077, 25741, 25959.

Fendlerella lasiopetala Standl. 16701, 18171, 18398, 20295, 21083, 21588, 24486, 27917.

Fendlerella utahensis (S. Watson) Heller. 18216, 18463, 18719, 25500, 29304.

Philadelphus calcicolus S.Y. Hu. 24093, 25262.

Philadelphus coulteri S. Watson. 17192, 17535, 18145.

Philadelphus karwinskyanus Koehne. 13342.

Philadelphus maculatus (C.L. Hitchc.) S.Y. Hu. 17584, 18712, 19029, 20445.

Philadelphus mexicanus Schltdl. 318, 2362, 5032.

Family Hydrocharitaceae

Najas guadalupensis (Spreng.) Magnus. 13596.

\section{Family Hydroleaceae}

Hydrolea spinosa L. 2575, 3790, 5123, 5373, 5725, 5806, 7246, 9788, 10396, 11331, 11743, 13512.

\section{Family Hydrophyllaceae}

Phacelia marshall-johnstonii N.D. Atwood \& Pinkava. 27465, 29586, 29605.

Phacelia vossii N.D. Atwood. 29898.

Phacelia congesta Hook. 18554, 18657, 18692, 19776, 20435, 21147, 28334.

Phacelia gypsogenia I.M. Johnst. 18185, 20280, 22314, 25719, 29283.

Phacelia hintoniorum B.L. Turner. 18223 (TYPUS), 27035, 27204.

Phacelia pallida I.M. Johnst. 16542.

Phacelia patuliflora (Engelm. \& A. Gray) A. Gray. 17036, 17808, 18851.

Phacelia platycarpa (Cav.) Spreng. 432, 614, 3854, 7956, 11862, 11961, 17366, 18311, 26535, 26664.

Phacelia potosina B.L. Turner. 18453 (TYPUS).

Phacelia robusta (J.F. Macbr.) I.M. Johnst. 21046.

Phacelia rupestris Greene. 19140, 20800, 21017, 22560.

Phacelia secunda J.F. Gmel. 17119, 17885, 18884.

Phacelia vossii N.D. Atwood. 18327, 19222, 21967, 22088, 22574, 23121, 23730, 25152, 27016, 27029, 27720, 28914.

Phacelia zaragozana B.L. Turner. 17868, Bis (TYPUS),
22439, 23322.

\section{Family Hypericaceae}

Hypericum formosum Kunth. 506, 865, 3801, 3859, 4189, 4208, 13014, 22292.

Hypericum gnidioides Seem. 7457.

Hypericum hypericoides (L.) Crantz. 24491, 24595, 24665.

Hypericum pauciflorum Kunth. 4771, 6729, 23150.

Hypericum philonotis Schltdl. \& Cham. 864, 1562, 8790, 10714, 15457.

Hypericum rubritinctum N. Robson. 9757 (TYPUS).

Hypericum silenoides Juss. 1361, 1796 (Type of Hypericum hintonii Bullock), 4600, 6518, 6731, 15365, 26352.

\section{Family Hypoxidaceae}

Hypoxis decumbens L. 22813, 22828, 22916, 23085.

Hypoxis mexicana Schult. \& Schult. f. 29737.

Hypoxis decumbens L. 9236, 13954, 17165, 24083, 24638.

Hypoxis fibrata Brackett. 11884.

Hypoxis humilis Kunth. 10559.

Hypoxis mexicana Schult. \& Schult. f. 13009, 23271, 23272.

Hypoxis tepicensis Brackett. 14388.

\section{Family Iridaceae}

Cipura paludosa Aubl. 1168, 1458, 6342, 8145, 9146, 10522, 10633, 13096, 15145.

Cypella mexicana C.V. Morton \& R.C. Foster. 6313, 10322 (TYPUS), 13907, 15959.

Iris versicolor L. 17888, 18209.

Nemastylis tenuis (Herb.) S. Watson. 17992.

Nemastylis tenuis (Herb.) S. Watson. 4741, 9163.

Orthrosanthus monadelphus Ravenna. 500, 3503, 8835, 13702, 15106, 25275.

Sisyrinchium schaffneri S. Watson. 18022.

Sisyrinchium tenuifolium Humb. \& Bonpl. ex Willd. 29713. Sisyrinchium angustifolium Mill. 785, 960.

Sisyrinchium angustissimum (B.L. Rob. \& Greenm.) Greenm. \& C.H. Thomps. 1112, 2529, 4252.

Sisyrinchium arguellesiae Ceja, Espejo \& López-Ferrari. 22954, 24080.

Sisyrinchium arizonicum Rothr. 1040, 4260, 4627, 20420, 20882.

Sisyrinchium biforme E.P. Bicknell. 24133.

Sisyrinchium cernuum (E.P. Bicknell) Kearney. 3617, 8923.

Sisyrinchium convolutum Nocca. 10473, 10560, 13011, 13926, 13950.

Sisyrinchium coulterianum Klatt ex Baker. 942, 4214.

Sisyrinchium elmeri Greene. 958.

Sisyrinchium hintoniorum G.L. Nesom. 18040, 22440 (TYPUS), 22959, 23975.

Sisyrinchium johnstonii Standl. 26795.

Sisyrinchium macrophyllum Greenm. 26087, 26663.

Sisyrinchium micranthum Cav. 21946, 27049.

Sisyrinchium microbracteatum G.L. Nesom. 21570, 23104, 23515, 23737 (TYPUS), 25662, 28135, 28704, 29060.

Sisyrinchium novoleonense G.L. Nesom \& L. Hern. 17298, 
17368, 18584, 23098, 23937.

Sisyrinchium platyphyllum S. Watson. 14445.

Sisyrinchium scabrum Cham. \& Schltdl. 11829, 11937, 14453, 16643, 18295, 19799, 20281, 21928, 22180, 23166, 23904, 24504, 25391, 25415.

Sisyrinchium schaffneri S. Watson. 17116, 18816, 18963, 20276, 21845, 22610, 22725.

Sisyrinchium tenuifolium Humb. \& Bonpl. ex Willd. 18536, 26161, 27599.

Sisyrinchium tolucense Peyr. 13018, 13173, 26670, 26736.

Tigridia multiflora (Herb.) Ravenna. 29712.

Tigridia coerulea Goldblatt. 13996 (Type of Sphenostigma hintonii R.C. Foster).

Tigridia flammea (Lindl.) Ravenna. 14432.

Tigridia fosteri Goldblatt. 8010 (Type of Sphenostigma mexicanum R.C. Foster).

Tigridia hallbergii Molseed. 9652.

Tigridia hintonii Molseed. 9748 (TYPUS).

Tigridia longispatha (Herb.) Goldblatt. 1401, 1453, 4614 (Type of Sphenostigma longispathum var. filiforme R.C. Foster), 13957.

Tigridia meleagris (Lindl.) G. Nicholson. 1477, 4174, 9036, 9933, 14429, 15977.

Tigridia mexicana Molseed. 6139.

Tigridia mortonii Molseed. 6527 (TYPUS).

Tigridia multiflora (Herb.) Ravenna. 9132, 9654, 9961, 10508, 15019, 15067, 15101, 17231, 23086, 23224.

Tigridia orthantha (Lem.) Ravenna. 26568, 26637.

Tigridia pavonia (L. f.) DC. 1432, 4524.

Tigridia seleriana (Loes.) Ravenna. 26543.

Tigridia vanhouttei Roezl ex Van Houtte. 1582, 4453, 4662.

\section{Family Juglandaceae}

Carya illinoinensis (Wangenh.) K. Koch. 18396, 19241.

Carya myristiciformis (Michx. f.) Nutt. 21374, 24191.

Carya ovata (Mill.) K. Koch. 23442, 24667, 24911, 25173.

Carya palmeri W.E. Manning. 24321, 25326.

Juglans hirsuta W.E. Manning. 22167.

Juglans major (Torr.) A. Heller. 3973 (Type of Juglans major var. glabrata W.E. Manning).

Juglans mollis Engelm. 23418, 24555, 24963, 25099.

Juglans pyriformis Liebm. 13759.

\section{Family Juncaceae}

Juncus acuminatus Michx. 3471.

Juncus aemulans Liebm. 26043, 26560.

Juncus balticus Willd. 22797.

Juncus bufonius L. 3525, 3702.

Juncus ebracteatus E. Mey. 1332, 1386, 1564.

Juncus effusus L. 3379, 3487, 15363.

Juncus microcephalus Kunth. 3726, 3905, 5601.

Juncus texanus (Engelm.) Cov. 22065.

Luzula caricina E. Mey. 14452.

Luzula denticulata Liebm. 1307, 15647, 26586.

Family Koeberliniaceae

Koeberlinia spinosa Zucc. 17844, 17866, 20082, 20600,
$20628,25885$.

\section{Family Krameriaceae}

Krameria bicolor S. Watson. 16503, 23012, 23200, 24736, $25165,25718,25781,27064,27104,27363,27456,28485$, 28826, 29137, 29240.

Krameria cytisoides Cav. 17285, 18694, 19283, 19613, 20963, 24063, 25159.

Krameria erecta Willd. ex Schult. 27687.

Krameria ramosissima (A. Gray) S. Watson. 24249.

Krameria secundiflora Moc. \& Sessé ex DC. 1196, 4326, 4483, 6301, 9069, 19583, 25358, 27094, 27101, 29317.

\section{Family Lamiaceae}

Agastache mexicana (Kunth) Link \& Epling. 2312, 15030. Agastache palmeri (B.L. Rob.) Standl. 17206, 17305, 18313, 18857, 20983, 22229, 27794.

Agastache pallidiflora (Heller) Rydb. 21244, 21491.

Asterohyptis mociniana (Benth.) Epling. 2188, 3004, 12582.

Asterohyptis stellulata (Benth.) Epling. 452, 2064, 2259, 5800, 6972, 11613, 11856, 12444, 13334, 15591.

Callicarpa acuminata Kunth. 24155, 24509, 24516, 24521, 24625, 24789, 25009.

Cantinoa americana (Aubl.) Harley \& J.F.B. Pastore. 2556, 5167, 12519, 14655.

Cantinoa mutabilis (Rich.) Harley \& J.F.B. Pastore. 1851, 2638, 2699, 11795, 12321, 12439, 12577, 12641, 12657, 12901, 13167, 13641, 14712, 15602, 17620, 23833, 24840, 25011, 25044, 25134.

Clerodendrum bungei Steud. 3879, 23517.

Clerodendrum coulteri (A. Gray) Govaerts. 23870, 27059.

Clerodendrum chinense (Osbeck) Mabb. 3442, 3880, 12697.

Clinopodium hintoniorum (B.L. Turner) Govaerts. 23059 (TYPUS), 23946, 27250, 27268, 27702.

Clinopodium macrostemum (Moc. \& Sessé ex Benth.) Kuntze. 523, 2452, 8930, 11933, 13707, 15676, 15755, 17481bis, 17482 bis, 26009.

Condea albida (Kunth) Harley \& J.F.B. Pastore. 12988, 15887.

Condea iodantha (Epling) Harley \& J.F.B. Pastore. 9844 (TYPUS).

Condea subtilis (Epling) Harley \& J.F.B. Pastore. 8574 (Type of Hyptis perpulchra Epling), 12605 (TYPUS), 12643, 16156.

Cunila lythrifolia Benth. 2448, 4885.

Cunila polyantha Benth. 14994, 26330.

Cunila pycnantha B.L. Rob. \& Greenm. 322, 3084, 3245, 5764, 8862, 11315.

Cunila ramamoorthiana M.R. García-Peña. 14866.

Hedeoma palmeri Hemsl. 22103, 22245, 22486, 24541, 24824, 25446, 25559, 25565.

Hedeoma ciliolata (Epling) R.S. Irving. 18088, 18632, 19292, 21502, 22176, 27019, 28721.

Hedeoma costata A. Gray. 16754, 17327, 17348, 18298, 18405, 20269, 21287, 21884, 21907, 22070, 22279, 22786, 22977, 23068, 24094, 24134, 24423, 25414, 25766, 27076, 
27228.

Hedeoma drummondii Benth. 17396, 17778, 18442, 19534, 20762, 21913, 27099, 27933, 29036, 29154.

Hedeoma irvingii B.L. Turner. 25974, 27082.

Hedeoma palmeri Hemsl. 17246, 17301, 17605, 18614, $19165,19185,21162,21246,21383,21686,21750,22159$, 22367, 27483.

Hedeoma patrina Stewart. 28263.

Hedeoma piperita Benth. 2095, 4925.

Hedeoma pusilla (R.S. Irving) R.S. Irving. 18524.

Hedeoma quercetora Epling. 22730.

Hyptis capitata Jacq. 13665.

Hyptis capitata Jacq. 2172.

Hyptis lantanifolia Poit. 10800, 14624.

Hyptis macrocephala M. Martens \& Galeotti. 10026, 12140, 15140.

Hyptis pseudolantana Epling. 11266 (TYPUS).

Leonotis nepetifolia (L.) R. Br. 15772, 19982, 21418, 23979.

Lepechinia caulescens (Ortega) Epling. 29735.

Lepechinia schiedeana (Schltdl.) Vatke. 22978.

Lepechinia caulescens (Ortega) Epling. 1551, 11964, 12362, 12388, 13109, 15515.

Lepechinia nelsonii (Fernald) Epling. 3078, 3299, 5765, 8832, 8866, 8970, 14961, 15399bis, 15399.

Lepechinia schiedeana (Schltdl.) Vatke. 24536.

Marrubium vulgare L. 1728, 3846, 16758, 17141, 17849, 19409.

Marsypianthes chamaedrys (Vahl) Kuntze. 9495, 10615.

Mentha canadensis L. 804, 4206, 7787.

Mentha suaveolens Ehrh. 3878, 4098, 17898, 17998, 27852.

Mesosphaerum involucratum (Benth.) Kuntze. 1597, 2146.

Mesosphaerum oblongifolium (Benth.) Kuntze. 7378, 9857, 9918, 11669, 12661, 12702, 12891, 14924, 15623.

Mesosphaerum pectinatum (L.) Kuntze. 2240, 2499, 3753, 5859, 12655, 12681.

Mesosphaerum septentrionale (Epling) Harley \& J.F.B. Pastore. 220, 2762, 2892, 5327, 8786, 16080.

Mesosphaerum suaveolens (L.) Kuntze. 2195, 4998, 11466, 11583, 12290, 12320, 14608, 14814, 15286, 15313, 26457. Mesosphaerum urticoides (Kunth) Kuntze. 169, 2261, 2502, 2994, 11277, 13381, 15386.

Monarda citriodora Cerv. ex Lag. 15444, 17125, 17250, 18514, 19576, 19808, 20401, 22086, 22324, 24210, 24919, 25389, 27958, 29160.

Monarda fistulosa L. 22142, 23204, 24188, 24598, 24669.

Monarda pringlei Fernald. 24110, 24336.

Ocimum basilicum L. 5826.

Ocimum campechianum Mill. 12124, 12288, 13897.

Physostegia correllii (Lundell) Shinners. 20536.

Plectranthus scutellarioides (L.) R. Br. 3950.

Poliomintha glabrescens A. Gray ex Hemsl. 16523.

Poliomintha longiflora A. Gray. 16704, 18747, 20428, 22073, 25556, 25807.

Prunella vulgaris L. 797, 1388, 11889, 11907, 11966, 18296, 21249, 22304, 22914.

Salvia carnea Kunth. 26049.
Salvia decora Epling. 12535.

Salvia elegans Vahl. 26773.

Salvia fulgens Cav. 13172.

Salvia indigocephala Ramamoorthy. 12792 (TYPUS).

Salvia iodantha Fernald. 12921.

Salvia jacobii Epling. 14110 (TYPUS), 14891.

Salvia laevis Benth. 29736.

Salvia languidula Epling. 14338.

Salvia longispicata M. Martens \& Galeotti. 11897bis, 12128.

Salvia mexicana L. 11239.

Salvia microphylla Kunth. 25073.

Salvia patriciae J.G. González \& E. Martínez-A. 26159 (TYPUS), 26651, 26787.

Salvia polystachia Cav. 18030, 29803.

Salvia protracta Benth. 26417.

Salvia prunelloides Kunth. 14323.

Salvia rostellata Epling. 14103.

Salvia sessei Benth. 9873.

Salvia sharpii Epling \& Mathias. 17568, 22892, 22904, 23127, 24877.

Salvia sirenis J.G. González \& G. González. 26385 (TYPUS).

Salvia stolonifera Benth. 26587.

Salvia subpatens Epling. 13989.

Salvia albocaerulea Linden. 585, 3510, 3552, 8852, 11806 , 13448, 17464bis.

Salvia arbuscula Fernald. 11699, 13722, 13729.

Salvia assurgens Kunth. 11858, 11960.

Salvia atropaenulata Epling. 13234, 15413.

Salvia azurea Lam. 17359.

Salvia ballotiflora Benth. 16593, 17314, 19599, 19649, 20781, 21053, 21131, 23051, 23180, 23894, 24199, 27635, 28540, 28702, 29447.

Salvia booleana B.L. Turner. 20340, 20519.

Salvia capillosa Epling. 13020 (TYPUS).

Salvia carnea Kunth. 795, 3542, 7473, 8684, 8938, 11074 , 11225, 11226, 13233, 14113, 14161, 14225, 14297, 14744, 15397.

Salvia caudata Epling. 23294.

Salvia cinnabarina M. Martens \& Galeotti. 9867, 11091, 14922, 14964, 26007, 26048, 26274, 26380.

Salvia clinopodioides Kunth. 1570, 13350, 15443.

Salvia coahuilensis Fernald. 17319, 18450, 18893, 20249, 21081, 22337, 22834, 23162, 24383, 25441, 28346.

Salvia coccinea Buc'hoz ex Etl. 13779, 17679, 19967, 20098, 20384, 20520, 21470, 23784, 24151, 24197, 24211.

Salvia compsostachys Epling. 23825, 24799.

Salvia concolor Lamb. ex Benth. 1309.

Salvia coulteri Epling. 19192, 20801, 25667, 25942, 27910.

Salvia cyanantha Epling. 15350 (TYPUS).

Salvia chalarothyrsa Fernald. 12171, 12329.

Salvia chamaedryoides Cav. 17468, 19064, 19796, 25508, 25969, 27091, 28997.

Salvia chionophylla Fernald. 16715.

Salvia decora Epling. 9762 (TYPUS).

Salvia dichlamys Epling. 914 (TYPUS), 9294, 10450, 11940, 
11988, 12192, 12410, 13001, 13787, 13892, 13916, 13949, 13956 (Type of Salvia nigriflora Epling).

Salvia dryophila Epling. 2700, 3064, 5406 (TYPUS).

Salvia elegans Vahl. 269, 311, 8909, 11085, 11810, 12773, 13453, 13706, 14298, 15404, 17488bis.

Salvia eplingiana Alziar. 11260 (TYPUS), 14940.

Salvia exilis Epling. 11093.

Salvia filipes Benth. 14900, 14921, 14980.

Salvia fluviatilis Fernald. 9482, 11897, 13774, 14674.

Salvia forreri Greene. 17103, 17929, 22273, 24642, 24697, 25400, 25420, 25486, 25562, 25602, 27249.

Salvia fulgens Cav. 15568.

Salvia gesneriflora Lindl. \& Paxton. 258, 267, 8805, 11809 , 13570, $15502,16064$.

Salvia glechomifolia Kunth. 14325, 14427, 29047.

Salvia gravida Epling. 12355 (TYPUS), 12397, 12799, 15371, 15677.

Salvia greggii A. Gray. 16821, 17982, 18305, 18490, 19601, 19901, 20090, 23139, 23677, 25504, 25805, 25933, 27096, 28935, 28979, 29010, 29018, 29106.

Salvia gypsophila B.L. Turner. 17470, 23136.

Salvia hamulus Epling. 9884 (TYPUS), 10728.

Salvia helianthemifolia Benth. 8828.

Salvia hintonii Epling. 9761, 10150 (TYPUS), 14094, 14096, 14888.

Salvia hispanica L. 2640, 3557, 4892, 5235, 12384, 14768, 14821, 15545.

Salvia involucrata Cav. 22562, 22660, 23792, 24603, 24675, 25086.

Salvia iodantha Fernald. 299, 2373, 2713, 2816, 9927, 11813, 12359, 12749, 12768, 12797, 13415, 13449, 13568, 14180, 14887, 14946, 15703, 16066, 16067, 16079.

Salvia jaimehintoniana Ramamoorthy ex B.L. Turner. 18615, 18840, 21276, 22285, 25880, 27233, 27234, 27920, 27946, 29046.

Salvia jessicae B.L. Turner. 29345, 29359 (TYPUS).

Salvia jorgehintoniana Ramamoorthy ex B.L. Turner. 21348 (TYPUS), 22456.

Salvia keerlii Benth. 23671, 23778, 28986.

Salvia laevis Benth. 963.

Salvia langlassei Fernald. 11703, 14105, 14162.

Salvia languidula Epling. 10350, 10351 (TYPUS), 12598, 14061, 14727, 16036.

Salvia lasiantha Benth. 11152, 14815.

Salvia lasiocephala Hook. \& Arn. 2123, 2654, 2675, 5304, 11004, 12330, 12648, 12715, 13292, 13433, 14132, 15296, 15690

Salvia lavanduloides Kunth. 134, 2470, 2772, 9878, 10838, 11693, 11811, 11953, 12370, 13474, 13557, 14740, 15258, 15354, 15442, 16081, 17477bis, 17496bis, 26006.

Salvia leninae Epling. 14897 (TYPUS).

Salvia leucantha Cav. 4886, 5223.

Salvia littae Vis. 26018, 26167, 26364, 26405.

Salvia longispicata M. Martens \& Galeotti. 3024, 15485, 20531.

Salvia longistyla Benth. 330, 2761, 2884, 11258, 13434.

Salvia lycioides A. Gray. 22067, 25561, 25627.

Salvia macellaria Epling. 20304, 20317, 22395, 22481.
Salvia melissodora Lag. 2840, 2906, 13045. Salvia mexicana L. 9005, 9912, 11702, 12394, 12402, 12705, 12872, 13348, 13539, 14878, 14889, 15437, 17468bis.

Salvia microphylla Kunth. 563, 912, 5237, 11861, 15285, 15451, 17024, 17375, 19881, 20132, 20807, 21092, 22266, 23942, 23949, 25421, 28207.

Salvia misella Kunth. 5472, 7521, 12583, 12637, 12837, 13201, 14672, 15287, 15630, 15904, 22905, 26459.

Salvia mocinoi Benth. 133, 2823, 3066, 8959, 11090, 13520, 14855, 14875, 15726.

Salvia modica Epling. 23413.

Salvia nana Kunth. 1096, 18922, 20424.

Salvia nepetoides Kunth. 4440, 8084, 13519.

Salvia nitida Benth. 14997.

Salvia novoleontis B.L. Turner. 22994.

Salvia oreopola Fernald. 16069.

Salvia oresbia Fernald. 24616.

Salvia palealis Epling. 14040 (TYPUS), 14971.

Salvia patens Cav. 4377, 16072.

Salvia perblanda Epling. 9946 (TYPUS).

Salvia plurispicata Epling. 13549, 15527.

Salvia polystachia Cav. 1346, 2605, 9550, 10310, 11062 , $11273,11833,12532,12533,12660,13241,13343,13379$, $14319,14754,14774,14854,14892,14948,15622,16078$, 17447 bis, 17477 bis.

Salvia potus Epling. 19259, 28289.

Salvia praestans Epling. 11095 (TYPUS), 14765.

Salvia protracta Benth. 2096, 12361, 12404, 14763, 14806. Salvia puberula Fernald. 11224 (Type of Salvia punicans Epling), 17485.

Salvia pubescens Benth. 334.

Salvia purpurea Cav. 2204, 2860, 3051, 11032, 11238, 11272, 11544, 12482, 13439, 14766, 14816, 16077.

Salvia reflexa Hornem. 16862, 17598, 18564, 19489, 20754, 21365, 24320, 27480, 27573, 27585, 27953.

Salvia regla Cav. 16810, 17259, 17572, 18650, 18769 , 19804, 20423, 21343, 21370, 23058, 23945, 24550, 27247, 27965, 28982.

Salvia reptans Jacq. 1438, 5374, 11918, 13043.

Salvia rhyacophila (Fernald) Epling. 2274, 9696, 11537, 14699.

Salvia roemeriana Scheele. 18413, 18616, 18768, 20053, 20260, 21073, 21589, 22035, 22769, 24189, 24366, 25029, 25941, 27874, 28235, 29058.

Salvia roscida Fernald. 12208, 12357, 12480.

Salvia sacculus Epling. 17668, 23069, 23094, 23147, 24645, 24722, 24798, 25192, 27259.

Salvia sapinea Epling. 14758, 14798 (TYPUS).

Salvia scaposa Epling. 1117, 5332 (TYPUS).

Salvia sessei Benth. 1589, 1788, 2073, 9702, 11150, 12812, 13033, 13160.

Salvia setulosa Fernald. 20736, 20737, 21352, 21712, 23052.

Salvia sharpii Epling \& Mathias. 27533.

Salvia stolonifera Benth. 14424, 14428, 14502.

Salvia subhastata Epling. 11714, 11715.

Salvia subobscura Epling. 12570 (TYPUS).

Salvia subpatens Epling. 4376 (TYPUS), 6234. 
Salvia synodonta Epling. 12576 (TYPUS), 12649.

Salvia teresae Fernald. 952, 999, 16075.

Salvia thyrsiflora Benth. 11236, 11321, 12411, 12769, 12930, 13610, 14016, 14092, 14101, 14102, 14963, 15608, 15744, 15769.

Salvia tiliifolia Vahl. 12398, 13054, 13182, 14819, 15539, 16068, 18637, 19937, 20636, 21364, 22452, 27248, 27954, 28162, 28981.

Salvia tubifera Cav. 15416.

Salvia unicostata Fernald. 17408, 22114, 22201, 22403, 22845, 23079, 27393.

Salvia urolepis Fernald. 19181, 19992, 20576, 21173, 21385 , 21458, 24954, 25126, 25904, 25943.

Salvia uruapana Fernald. 15217.

Salvia viscidifolia Epling. 10725 (TYPUS), 12369, 15091.

Salvia xalapensis Benth. 16073.

Salvia zaragozana B.L. Turner. 22382 (TYPUS), 22529, 22589.

Scutellaria fruticetorum Epling. 25311.

Scutellaria macra Epling. 24781.

Scutellaria aramberrana B.L. Turner. 18999 (TYPUS), 23169.

Scutellaria blepharophylla Epling. 1086, 10507 (TYPUS), 13058, 13893.

Scutellaria drummondii Benth. 17779.

Scutellaria dumetorum Schltdl. 1310, 4638, 8259, 9574, 11903, 13027, 13991, 14506, 15005, 15375, 15982, 17220, 17626, 23093, 26553.

Scutellaria formosa Leonard. 11073.

Scutellaria hintoniana Epling. 996, 1136 (TYPUS), 4641, 8275.

Scutellaria hintoniorum Henr. 18213 (TYPUS), 25800.

Scutellaria hispidula B.L. Rob. 21883, 21994, 22160.

Scutellaria longifolia Benth. 11041, 11188.

Scutellaria lutilabia T.M. Lane \& G.L. Nesom. 21760, 22318, 22357, 22570, 23026, 29140.

Scutellaria monterreyana B.L. Turner. 24123, 24220, 24419 , 25931.

Scutellaria multiflora Benth. 5319.

Scutellaria potosina Brandegee. 16638, 17011, 17351, 18507, 19904, 20236, 20527, 20964, 21450, 22024, 22091, 22620, 24356, 24506, 25351, 25442, 25597, 25935, 27395, 27397, 27646, 27858, 27940, 27989, 29057, 29196.

Scutellaria seleriana Loes. 17961, 24445, 24788.

Scutellaria suffrutescens S. Watson. 19154, $24192,24643$.

Stachys agraria Schltdl. \& Cham. 29841.

Stachys nepetifolia Desf. 18001.

Stachys agraria Schltdl. \& Cham. 853, 1175, 3538, 3539, 6175, 9289, 10550, 11878, 13894, 24111, 24345.

Stachys bigelovii A. Gray. 18509, 18723, 19711, 19781, 19907, 20243, 21094, 21126, 21305, 21616, 21919, 21993, 22102, 22289, 25913, 27261, 27264.

Stachys boraginoides Cham. \& Schltdl. 14108, 15501.

Stachys coccinea Ortega. 270, 1108, 2016, 2807, 5896, 8283, 8952, 8993, 9730, 11096, 11110, 11160, 11384, 11816, 16058, 16255, 17478bis, 26014, 26174, 26201, 26401.

Stachys eriantha Benth. 18520.

Stachys grahamii Benth. 1387, 3989, 13177, 23508, 24399,
24561, 24639.

Stachys hintoniorum B.L. Turner. 18294 (TYPUS), 18885. Stachys jaimehintonii B.L. Turner. 13489 (TYPUS).

Stachys langmaniae Rzed. \& Calderón. 17419, 17545 (TYPUS), 22542, 22973.

Stachys pacifica B.L. Turner. 15319 (TYPUS).

Stachys radicans Epling. 1898, 8006.

Stachys sanchezii Rzed. \& A. García. 26530, 26700, 26812.

Stachys tamaulipana B.L. Turner. 24613.

Stachys vulnerabilis Rzed. \& Calderón. 17201, 17307, 24774, 25333.

Teucrium canadense L. 17826.

Teucrium cubense Jacq. 16644, 17126, 17636, 19414, 19496, 20062, 20323, 24130, 24434, 25899, 27588.

Teucrium vesicarium Mill. 20328.

Vitex hemsleyi Briq. 13789.

Vitex mollis Kunth. 450, 3215, 3384, 3752, 5704, 9970, 9971, 10989, 14121.

Vitex pyramidata B.L. Rob. 720, 740, 3829, 4086, 5999, 6956, 9990, 10002, 10005, 11244.

\section{Family Lauraceae}

Damburneya longicaudata (Lundell) Trofimov \& Rohwer. 13791.

Damburneya martinicensis (Mez) Trofimov. 10589, 11473, 16215.

Damburneya nitida (Mez) Trofimov \& Rohwer. 13835, 13918.

Damburneya salicifolia (Kunth) Trofimov \& Rohwer. 13667, 13668, 13856.

Licaria cervantesii (Kunth) Kosterm. 10194, 10273, 10290, 10291.

Litsea glaucescens Kunth. 3188, 3257, 7223, 12800, 15418, 15746, 17491, 17493, 22630, 22640, 22728, 22831, 24554, 24899.

Litsea muelleri Rehder. 17280, 18133, 18384, 19344, 20182, 21811, 27975.

Litsea parvifolia (Hemsl.) Mez. 21872, 25228, 28068.

Litsea pringlei Bartlett. 25200.

Ocotea acuminatissima (Lundell) Rohwer. 332.

Ocotea betazensis (Mez) van der Werff. 14014.

Ocotea salvinii Mez. 3083, 3816, 15837, 16250.

Ocotea tampicensis (Meisn.) Hemsl. 17747, 24623.

Persea americana Mill. 3167, 6215, 22632, 25042, 25203, 25257, 25258.

Persea hintonii C.K. Allen. 3533 (TYPUS), 3980, 14027, 14195.

Persea liebmannii Mez. 16903, 25199.

\section{Family Lentibulariaceae}

Pinguicula caudata Schltdl. 11989.

Pinguicula colimensis McVaugh \& Michel. 9885.

Pinguicula crenatiloba A. DC. 8609.

Pinguicula cyclosecta Casper. 17641, 21309, 22507, 22539, 22749, 22966, 23080, 23125, 23240, 23257, 23511.

Pinguicula ehlersiae Speta \& F. Fuchs. 19312, 22661 (Type of Pinguicula hintoniorum B.L. Turner). 
Pinguicula esseriana B. Kirchner. 25195, 25202.

Pinguicula gracilis Zamudio. 19349, 22794, 24044, 24045.

Pinguicula heterophylla Benth. 955, 4049, 4166, 10417, 13839, 14322.

Pinguicula imitatrix Casper. 14219 (TYPUS).

Pinguicula immaculata Zamudio \& Lux. 19294, 20191, 23650, 23763.

Pinguicula kondoi Casper. 19021, 21936, 22590, 22700, 22716, 28233.

Pinguicula macrophylla Kunth. 17164, 24303.

Pinguicula moranensis Kunth. 843, 2924, 7975, 11866, 15107, 26166, 26377, 26609, 26612, 26694.

Pinguicula orchidioides DC. 14504.

Pinguicula rotundiflora Studnicka. 10682, 21812, 24000

(Type of Pinguicula jorgehintonii B.L. Turner), 28188.

Pinguicula zecheri Speta \& Fuchs. 14442.

Utricularia hintonii P. Taylor. 4960 (TYPUS).

Utricularia livida E. Mey. 625, 8295, 9457.

Utricularia pusilla Vahl. 5267.

\section{Family Liliaceae}

Calochortus barbatus (Kunth) J.H. Painter. 26676.

Calochortus fuscus Schult. f. 1383 (Type of Calochortus hintonii Bullock ex Ownbey), 1441, 1512, 1682, 1824, 4739, 4752, 9330, 12413, 13354, 15136.

Calochortus marcellae G.L. Nesom. 17630, 19172, 20723, 21525, 22533, 23246, 23316, 23764, 24882, 28703, 28901.

Calochortus pringlei B.L. Rob. 4863.

Calochortus purpureus (Kunth) Baker. 13157.

Calochortus venustulus Greene. 1687, 1841, 11200bis, 11204.

\section{Family Linaceae}

Linum lewisii Pursh. 29628.

Linum lasiocarpum Rose. 18725, 24232, 24409.

Linum lewisii Pursh. 17007, 18846, 20211, 20476, 20759 , 22400, 27037, 27593.

Linum mexicanum Kunth. 1355, 1849, 2027, 4873, 6404, 8788, 10664, 26660, 26681.

Linum modestum C.M. Rogers. 18137, 18196, 18240, 18759, 19293, 20155, 25646, 28034, 28708, 28908.

Linum orizabae Planch. 807, 1345, 14796.

Linum pringlei S. Watson. 23256, 28522.

Linum rupestre (A. Gray) Engelm. ex A. Gray. 16507, 18526, 19311, 20411, 21970, 22322, 22697, 22708, 23115, 23592, 23743, 24246, 24254, 25703, 27015, 27374, 29179, 29465. Linum scabrellum Planch. 19106, 22320, 22707, 23043, 25371, 27692, 28553, 29003.

Linum schiedeanum Schltdl. \& Cham. 15366, 17562, 18971, 19468, 19692, 21307, 22270, 22305, 24915, 25946, 27190. Linum vernale Wooton. 17662, 27060, 29135.

\section{Family Linderniaceae}

Lindernia anagallidea (Michx.) Pennell. 14587.

Lindernia blancoi (Merr.) Philcox. 14571.

Lindernia dubia (L.) Pennell. 3255, 5201, 5729, 5866.

Family Loasaceae
Cevallia sinuata Lag. 16595, 18095, 18329, 18456, 19289, 19341, 19978, 20935, 22334, 22814, 23570.

Eucnide durangensis H.J. Thomps. \& Powell. 23534, 27466.

Eucnide hirta (G. Don) H.J. Thomps. \& W.R. Ernst. 5895. Eucnide lobata (Hook.) A. Gray. 7562, 16675, 17821, 18640, 19274, 19334, 19348, 20930, 21047, 21208, 21715, 21835, 22157, 22764, 23103, 23114, 23568, 23705, 23752, 24418, 24764, 25961, 28252, 28364, 28381, 28628, 28873, 28885, 29305, 29405, 29591.

Eucnide xylinea C.H. Müll. 24145, 24365.

Gronovia longiflora Rose. 2173, 5121, 5164, 13131.

Gronovia scandens L. 1267, 6430, 6897, 13305, 14553.

Klaprothia fasciculata (C. Presl) Poston. 1773, 4833, 14632.

Mentzelia hintoniorum B.L. Turner \& Hempel. 29887.

Mentzelia hispida Willd. 18011.

Mentzelia aspera L. 1785, 2473, 5000, 5125, 10958, 15194.

Mentzelia asperula Wooton \& Standl. 23610.

Mentzelia gypsophila B.L. Turner. 28508, 28784 (TYPUS). Mentzelia hintoniorum B.L. Turner \& Hempel. 25495 (TYPUS), 25879, 27127.

Mentzelia hispida Willd. 5129, 16850, 17655, 18698, 19194, 19762, 20772, 21586, 21646, 22561, 22569, 23336, 23537, 23633, 23643, 23738, 24213, 24849, 25901, 27237, 27370, 27685, 27768, 27771, 27774, 28457, 28507, 28923.

Mentzelia mexicana H.J. Thomps. \& Zavort. 23347, 23562, 25958, 28353, 28408, 28444, 28640, 29403, 29412, 29421. Mentzelia multiflora (Nutt.) A. Gray. 19485bis, 27621.

Mentzelia pachyrhiza I.M. Johnst. 21996, 27423, 29397, 29414.

Mentzelia reverchonii (Urb. \& Gilg) H.J. Thomps. \& Zavort. 18301, 19447.

Mentzelia saxicola H.J. Thomps. \& Zavort. 27839.

Petalonyx crenatus A. Gray. 28359, 28459, 29308, 29364.

\section{Family Loganiaceae}

Mitreola petiolata (J.F. Gmel.) Torr. \& A. Gray. 2629, 5005, 6949, 11572.

Spigelia mexicana A. DC. 4477.

Spigelia nicotianiflora Chodat \& Hassler. 8063.

Spigelia scabrella Benth. 1380, 1404, 4469, 13982, 15000, 15043.

Spigelia speciosa Kunth. 10371.

Spigelia trispicata H. Hurley ex K.R. Gould. 10381 (TYPUS).

\section{Family Loranthaceae}

Cladocolea loniceroides (Tiegh.) Kuijt. 10458, 10500.

Cladocolea stricta Kuijt. 10689.

Cladocolea coyucae Kuijt. 3958 (TYPUS), 6688.

Cladocolea grahamii (Benth.) Tiegh. 3107, 3550, 9021, 10149.

Cladocolea hintonii Kuijt. 10148 (TYPUS).

Cladocolea microphylla (Kunth) Kuijt. 3796, 7689, 15022.

Cladocolea oligantha (Standl. \& Steyerm.) Kuijt. 4730, 8146. 
Cladocolea pedicellata Kuijt. 4091 (TYPUS).

Cladocolea stricta Kuijt. 10176 (TYPUS).

Psittacanthus calyculatus (DC.) G. Don. 827, 896, 2559, 2961.

Psittacanthus palmeri (S. Watson) Barlow \& Wiens. 2612. Psittacanthus rhynchanthus (Benth.) Kuijt. 10086, 10404. Struthanthus interruptus (Kunth) G. Don. 1281.

\section{Family Lythraceae}

Ammannia auriculata Willd. 6884.

Cuphea cyanea DC. 7202.

Cuphea wrightii A. Gray. 29718.

Cuphea aequipetala Cav. 659, 675, 8246, 11824, 11990, 12721, 13880, 15231, 16814, 17399, 20713, 25409, 26003, 26716.

Cuphea angustifolia Jacq. ex Koehne. 6004.

Cuphea appendiculata Benth. 9228, 10636, 11028, 12193, 14358, 14515, 14626, 15033.

Cuphea avigera B.L. Rob. \& Seaton. 1958, 2691 (Type of Cuphea hintonii Bullock), 3408, 7008, 7069, 7079, 9691.

Cuphea bracteolosa Koehne. 10799.

Cuphea bustamanta Lex. 502, 964, 3048, 7955, 8429, 11084 , 11129, 11853, 14041, 14275, 15721, 17158, 26438.

Cuphea calaminthifolia Schltdl. 12354, 15021, 15355.

Cuphea cyanea DC. 107, 1688, 2364, 4036, 5278, 6848, 12406, 12761, 17405, 19145, 20433, 21347, 22128, 24298, 24672, 25055.

Cuphea empetrifolia Rose. 14098.

Cuphea heterophylla Benth. 1116, 1184, 1294, 1540, 9210, 10377, 10491, 13042, 14375, 14884.

Cuphea hookeriana Walp. 672, 3628, 7453, 9729, 9860, 9861, 9882, 9942, 11101, 11169, 11235, 12465, 12724, 12790, 13426, 13701, 14330, 14838, 14858, 14923, 15171, $15687,15717$.

Cuphea jorullensis Kunth. 569, 842, 4913, 7947, 13017.

Cuphea koehneana Rose. 4541, 14493.

Cuphea laminuligera Koehne. 1174, 9195, 9410, 9525, 13211, 13212, 13311, 14570, 15180.

Cuphea lanceolata W.T. Aiton. 19994, 20002, 21439, 22460, 22586, 23803, 24872.

Cuphea leptopoda Hemsl. 1457, 6456, 12043, 12120, 13095, 14527.

Cuphea lobophora Koehne. 986, 1072, 1130, 2466, 4681, 6249, 6548, 7511, 7643, 7904, 9196, 9446, 10365, 10830, 13038, 14387.

Cuphea llavea Lex. 12127 (TYPUS), 12215, 15998, 15999, 16159.

Cuphea micropetala Kunth. 1495, 4007, 5136, 8373, 12129 , 12334, 15293.

Cuphea michoacana R.C. Foster. 12268 (TYPUS), 12269.

Cuphea nudicostata Hemsl. 26311, 26339, 26369.

Cuphea ornithoides R.C. Foster. 9859, 9863, 10678 (TYPUS).

Cuphea paucipetala S.A. Graham. 1523 (TYPUS), 13974.

Cuphea pertenuis R.C. Foster. 2504, 2790, 5113, 7083, 8361, 8545, 9717, 12845, 13460 (TYPUS).

Cuphea procumbens Ortega. 1576, 1829, 2151, 4417, 7081, 8292, 11894, 11916, 12139, 13102, 13111, 15174, 15440.
Cuphea pulcherrima R.C. Foster. 9850, 11157, 11274, 14813, 14837 (TYPUS).

Cuphea trichochila R.C. Foster. 9583 (TYPUS).

Cuphea trochilus S.A. Graham. 15333.

Cuphea utriculosa Koehne. 11025, 11595, 12667.

Cuphea vesiculigera R.C. Foster. 4478, 8164, 9166, 10570 (TYPUS).

Cuphea wrightii A. Gray. 1350, 1418, 1620, 9340, 10694, 12044, 12218, 13101, 15125 (Type of Cuphea wrightii var. compacta R.C. Foster).

Heimia salicifolia Link. 356, 3675, 5418, 11649, 11819, 12524, 12839, 17669, 19938, 22984, 23985, 24532.

Lafoensia punicifolia DC. 10817, 10822.

Lagerstroemia indica L. 811, 3951.

Lawsonia inermis L. 3768, 5479, 9040.

Lythrum californicum Torr. \& A. Gray. 17862, 20537.

Lythrum vulneraria Aiton ex Schrank. 484, 1225, 4249, 7784, 11826.

Punica granatum L. 9032.

Rotala ramosior (L.) Koehne. 4444, 4956, 5502, 5505.

Family Magnoliaceae

Magnolia tamaulipana A. Vázquez. 24328, 25208.

\section{Family Malpighiaceae}

Aspicarpa hyssopifolia A. Gray. 23622.

Bunchosia biocellata Schltdl. 13190, 25308.

Bunchosia lindeniana A. Juss. 15168, 16101, 16280.

Bunchosia nitida (Jacq.) DC. 9342, 10328, 13942.

Bunchosia palmeri S. Watson. 1618, 3430, 4134, 6013, 6539, 7148, 7265.

Byrsonima crassifolia (L.) Kunth. 445, 717, 3324, 4382, 5950, 9984, 10200, 10441, 13887, 14129.

Calcicola sericea (Nied.) W.R. Anderson \& C. Davis. 16535, 23369, 27439, 28465, 29129.

Callaeum coactum D.M. Johnson. 12962.

Callaeum macropterum (Sessé \& Moc. ex DC.) D.M. Johnson. 5445, 9967, 11151, 17590.

Callaeum malpighioides (Turcz.) D.M. Johnson. 14871.

Callaeum septentrionale (A. Juss.) D.M. Johnson. 17788, 19432, 21137, 21228, 21535, 22187, 24160, 24314.

Cottsia gracilis (A. Gray) W.R. Anderson \& C. Davis. 28410.

Echinopterys eglandulosa (A. Juss.) Small. 3179, 3756, 7399.

Echinopterys setosa Brandegee. 16513, 25739, 29241, 29342.

Galphimia glandulosa Cav. 3195, 3411, 5749, 7354, 7528. Galphimia glauca Cav. 2433, 2546, 7364, 8039, 9372, 9848, 13220, 15684, 24313, 24570, 24875, 24884.

Galphimia gracilis Bartl. 313, 12893, 13435, 15867, 16245.

Galphimia paniculata Bartl. 10124, 11097, 11292, 11758, 12694.

Galphimia tuberculata (Rose) Nied. 11683.

Galphimia vestita S. Watson. 13286.

Gaudichaudia albida Schltdl. \& Cham. 11495.

Gaudichaudia cycloptera (DC.) W.R. Anderson. 11253. 
Gaudichaudia cynanchoides Kunth. 1695, 1704, 2289, 5400, 10795, 12488, 13256, 15132.

Gaudichaudia diandra Chodat. 9523.

Gaudichaudia mollis Chodat. 1170, 1634, 13091.

Gaudichaudia mucronata (Moc. \& Sessé ex DC.) A. Juss. 15447.

Heteropterys brachiata (L.) DC. 1385, 2075, 2135, 2255, 2515, 2547, 2959, 3615, 5398, 6941, 7213, 9478, 11592, 12549, 12903, 15788.

Heteropterys cotinifolia A. Juss. 3003, 6496, 6867.

Heteropterys laurifolia (L.) A. Juss. 14141.

Heteropterys palmeri Rose. 6544, 10854, 10954.

Malpighia glabra L. 6132, 7857, 10933, 17678.

Malpighia hintonii Bullock. 1192 (TYPUS), 4489, 4736bis, 4736.

Malpighia mexicana A. Juss. 7071, 8051, 9104, 10468, 10609, 13035, 14392.

Mascagnia lilacina (S. Watson) Nied. 16818, 20809, 20844, 21065, 21156, 23364, 24447, 25633, 25856, 25954, 27468.

Mascagnia polybotrya (A. Juss.) Nied. 2595, 2631, 6990, 13272.

Psychopterys dipholiphylla (Small) W.R. Anderson \& S. Corso. 3147, 3314, 3370, 5569, 5744, 7498, 7513, 7523.

Tetrapterys mexicana Hook. \& Arn. 3020, 3237, 3354, 3363, 5834, 7093, 7500, 11760.

Tetrapterys schiedeana Schltdl. \& Cham. 5182, 6318.

\section{Family Malvaceae}

Abelmoschus esculentus (L.) Moench. 4727.

Abutilon abutiloides (Jacq.) Garcke ex Hochr. 17734.

Abutilon andrieuxii Hemsl. 11783, 16214.

Abutilon bracteosum Fryxell. 11010.

Abutilon coahuilae Kearney. 27436.

Abutilon divaricatum Turcz. 7431 (Type of Abutilon divaricatum var. hintonii Fryxell).

Abutilon fruticosum Guill. \& Perr. 27516.

Abutilon hypoleucum A. Gray. 20035, 20845, 21998, 23874 , 25220, 27783.

Abutilon malacum S. Watson. 23181, 23565, 24286.

Abutilon simulans Rose. 3586, 5046.

Abutilon wrightii A. Gray. 22873, 27814.

Alcea rosea L. 5224, 6127.

Allosidastrum pyramidatum (Cav.) Krapov., Fryxell \& D.M. Bates. 2647, 5684, 12848.

Allowissadula holosericea (Scheele) D.M. Bates. 21401, 21484, 21661.

Anoda cristata (L.) Schltdl. 29798, 29853.

Anoda acerifolia Cav. 10885, 12230, 12847, 13205, 14597. Anoda albiflora Fryxell. 19873.

Anoda cristata (L.) Schltdl. 301, 1011, 1154, 1215, 2058, 2564, 5225, 6796, 6877, 6889, 6987, 7464, 8426, 9612, 11165, 11359, 14824, 15328, 21487, 21489, 23415, 24874. Anoda hintoniorum Fryxell. 7229, 12778 (TYPUS).

Anoda paniculata Hochr. 1926, 5025, 6923.

Anoda pentaschista A. Gray. 5857, 6697.

Anoda pubescens Schltdl. 3036, 9524, 12523, 13554.

Anoda thurberi A. Gray. 25672.

Ayenia berlandieri S. Watson. 6861, 6928, 13224.
Ayenia magna L. 6655, 6859, 6919, 8160 Ayenia palmeri S. Watson. 12118, 15193. Ayenia purpusii Brandegee. 12082 (Type of Ayenia pringlei Cristóbal).

Ayenia pusilla L. 6678, 14615, 24751. Ayenia sidifolia (Turcz.) Hemsl. 2193.

Ayenia wrightii B.L. Rob. 16211.

Bastardiastrum hirsutiflorum (K. Presl) D.M. Bates. 14836. Bastardiastrum tricarpellatum (B.L. Rob. \& Greenm. ex Rose) Bates. 3402, 7240.

Batesimalva violacea (Rose) Fryxell. 17601, 19209.

Briquetia spicata (Kunth) Fryxell. 6601, 10961, 12651.

Byttneria aculeata (Jacq.) Jacq. 2577, 4355, 5195, 5522, 5662, 6982, 7098, 10649, 10806, 10819, 11362, 11431, 12032, 12278, 14545, 14609, 14681, 15201.

Byttneria atrata Bullock. 1242, 1378, 4664, 4711, 4830 (TYPUS), 6414, 11664.

Byttneria catalpifolia Jacq. 11590, 12286, 12634, 14567. Callianthe striata (Dicks. ex Lindl.) Donnell. 3566, 4878. Callirhoe involucrata (Nutt. ex Torr. \& A. Gray) A. Gray. 17771, 18541, 19872, 21190, 25390.

Ceiba aesculifolia (Kunth) Britten \& Baker f. 5711, 5727, 7150, 7271, 7920, 13878.

Corchorus hirtus L. 2034, 6371, 6463, 6939, 11398, 13074, 13078, 13193.

Chiranthodendron pentadactylon Larreat. 26093, 26279, 26292.

Dendrosida sharpiana (Miranda) Fryxell. 11515 (Type of Dendrosida sharpiana subsp. occidentalis Fryxell).

Fuertesimalva limensis (L.) Fryxell. 29777.

Gossypium barbadense L. 839, 3111, 5288, 5423, 7174, 8586.

Gossypium hirsutum L. 5133, 5444, 6103, 6644, 7102, 11333, 26462.

Gossypium trilobum (DC.) Skovsted. 13253.

Guazuma ulmifolia Lam. 692, 745, 3635, 5679, 6714, 6765, 6769, 6954, 7142, 7160, 9043, 9073, 9976, 10425, 10891, 13860, 14124, 14554.

Hampea tomentosa (C. Presl) Standl. 11374.

Helicteres guazumifolia Kunth. 1064, 3407, 10367, 10818, 11361, 11637, 14140, 14643.

Heliocarpus occidentalis Rose. 1768, 1917, 2022, 4265, 4791, 6605, 6721, 6724, 6876, 6901, 6906, 6950, 6951, 9801, 9802, 10901, 10943, 15252.

Heliocarpus pallidus Rose. 2843, 5109, 5369, 5416, 7036, 7155, 7236, 11267.

Heliocarpus terebinthinaceus (DC.) Hochr. 2723, 4953, 5045, 5119, 6736, 7077, 7243, 12449, 12477, 13246, 14831.

Heliocarpus velutinus Rose. 13398.

Herissantia crispa (L.) Brizicky. 3915, 4022, 5055, 5155, 10982, 11206, 15326, 19242, 23717, 23876, 27747, 28841.

Hermannia inflata Link \& Otto. 13274.

Hermannia texana A. Gray. 24290.

Hibiscus acicularis Standl. 17967, 20544, 20769, 28549.

Hibiscus coulteri Harvey ex A. Gray. 22781, 23177, 25790, 25979, 27821, 28411.

Hibiscus denudatus Benth. 23184, 24747, 28440, 29237.

Hibiscus elegans Standl. 27691. 
Hibiscus jaliscensis Fryxell. 7578.

Hibiscus martianus Zucc. 20822, 21457, 23614, 23696, 25221, 25887, 27752, 28538.

Hibiscus poeppigii (Spreng.) Garcke. 25223.

Hibiscus radiatus Cav. 1915, 2156, 5013, 9633.

Hibiscus rosa-sinensis L. 6211.

Hibiscus uncinellus DC. 11184, 11687.

Hochreutinera amplexifolia (DC.) Fryxell. 11630, 12325, 14682.

Kearnemalvastrum lacteum (Aiton) D.M. Bates. 658, 3664, 4939, 6818, 8319.

Kearnemalvastrum subtriflorum (Lag.) D.M. Bates. 1897, 2914, 4884, 6791, 7179, 12385, 13496, 26204.

Kosteletzkya blanchardii Fryxell. 13338.

Kosteletzkya depressa (L.) O.J. Blanch., Fryxell \& D.M. Bates. 2607, 5031, 7025.

Kosteletzkya tubiflora (DC.) O.J. Blanch. \& McVaugh. 1964, 2253, 4843, 5018, 9600, 9781, 11554, 12483, 15280.

Lopimia malacophylla (Link. \& Otto) Mart. 11197.

Luehea candida (Moc. \& Sessé ex DC.) Mart. 6099, 9138, 14347.

Malachra alceifolia Jacq. 5210, 5496, 12877.

Malachra capitata (L.) L. 12154.

Malachra fasciata Jacq. 11529, 12525, 16184.

Malva neglecta Wallr. 4578, 5021.

Malva parviflora L. 19411, 19543, 20473.

Malvastrum americanum (L.) Torr. 23773, 25224.

Malvastrum coromandelianum (L.) Garcke. 14558, 23328.

Malvaviscus arboreus Cav. 663, 3861, 4014, 4563, 5145, 5254, 6719, 7184, 9597, 10753, 10897, 13529.

Malvaviscus penduliflorus DC. 11030, 14181.

Malvaviscus urticifolius (C. Presl) Fryxell. 690, 3928 (Type of Malvaviscus hintonii Bullock), 4289, 5057, 5371, 7912.

Malvella lepidota (A. Gray) Fryxell. 24859, 27360.

Malvella leprosa (Ortega) Krapov. 17896.

Melochia corymbosa (C. Presl) Meisn. ex Steud. 3429, 4338, 5380, 7294, 12957, 14472.

Melochia nodiflora Sw. 1777, 2072, 2144, 2208, 2950, 4675, 5368, 5689, 9640, 11024, 13407, 15282.

Melochia nudiflora Standl. \& L.O. Williams. 10908, 15232, 26490.

Melochia pyramidata L. 1742, 2981, 4018, 5622, 13073, 17651, 17790, 21546, 24148, 24223, 24627.

Melochia spicata (L.) Fryxell. 1372, 1643, 2958, 5623, 7285, 11371, 12206, 14343.

Melochia tomentosa L. 12121, 13064, 24179, 26464.

Meximalva filipes (A. Gray) Fryxell. 20784, 23721, 23867, 24271.

Modiola caroliniana (L.). G. Don. 4072.

Neobrittonia acerifolia (G. Don) Hochr. 154, 424, 2305, 6856, 7178, 13538.

Pavonia lasiopetala Scheele. 16899, 24437.

Pavonia nepetifolia (Standl.) Standl. 18881, 23405, 29022.

Pavonia oxyphylla (DC.) Fryxell. 8579, 13341, 13368.

Pavonia pleuranthera (DC.) Fryxell. 12717.

Pavonia schiedeana Steud. 14516.

Pavonia uniflora (Sessé \& Moc.) Fryxell. 24515, 24779, 24825
Periptera macrostelis Rose. 11700.

Periptera punicea (Lag.) DC. 285, 2400, 2701, 5581, 5587, 6808, 6997, 7015, 7216, 11159, 12804, 13441.

Phymosia rosea (DC.) Kearney. 621, 3035, 3800, 4109, 5421, 5593, 5597, 5781, 7468, 7469, 7471, 7481, 8632, 8946.

Physodium adenodes (Goldberg) Fryxell. 16203 (TYPUS).

Pseudabutilon ellipticum (Schltdl.) Fryxell. 2916, 2943, 5134, 7028, 7674, 15722, 23481.

Pseudobombax ellipticum (Kunth) Dugand. 3214, 3234, 3403, 5706, 5910, 7267, 7312, 7601, 7628, 7631, 7696, 7726, 7823, 7885, 7914, 9983.

Robinsonella hintonii Fryxell. 7321 (TYPUS).

Sida linearis Cav. 29856.

Sida abutifolia Mill. 4466, 5461, 6432, 6966, 13083, 16645, 20370, 20618, 20760, 22870, 23183, 23379, 23544, 25890, 27519, 28921.

Sida acuta Burm. f. 2238, 5094, 15306, 24626, 24778.

Sida aggregata C. Presl. 2899, 7353, 7422, 12897.

Sida ciliaris L. 6490, 15181.

Sida glabra Mill. 2544, 2708, 2979, 5047, 5157, 6918, 12905.

Sida haenkeana C. Presl. 12490, 15484.

Sida hyssopifolia C. Presl. 19240.

Sida jamaicensis L. 9531.

Sida jussieana DC. 14728.

Sida lindheimeri Engelm. \& A. Gray. 20595.

Sida linifolia Cav. 2239, 6534, 11397.

Sida neomexicana A. Gray. 27782, 28725.

Sida rhombifolia L. 109, 471, 1685, 1770, 4033, 4659, 5721, 5852, 10745, 13136, 17750, 24221, 24839.

Sida salviifolia C. Presl. 6448.

Sida spinosa L. 9787, 11445, 17684, 20542, 24222.

Sphaeralcea angustifolia (Cav.) G. Don. 16616, 17730, 17843, 19516, 19570, 23350, 24362, 28888.

Sphaeralcea endlichii Ulbrich. 16617, 23351, 23530, 25968, 27433, 28509, 28782.

Sphaeralcea hastulata A. Gray. 19426, 19491, 19629, 27557, 27569.

Theobroma cacao L. 7531.

Tilia americana L. 820, 1340, 2030, 4120, 8277, 10405, 10475, 15480, 22147.

Trichospermum galeottii (Turcz.) Kosterm. 9902, 10272 , 10980, 11183, 11579, 11777, 16145, 16300.

Triumfetta bogotensis DC. 2021.

Triumfetta columnaris Hochr. 4917 (Type of Triumfetta columnarioides Bullock).

Triumfetta galeottiana Turcz. 13340, 14371, 14638, 15281, 15681 .

Triumfetta mexiae C.V. Morton \& Lay. 6621, 13230.

Triumfetta paniculata Hook. \& Arn. 11602.

Triumfetta simplicifolia (Sessé \& Moc.) Fryxell. 3837, 4224, 5324, 7027, 12343.

Triumfetta stellata Lay. 4460 (TYPUS).

triumfetta acracantha Hochr. 10911.

Triumfetta bogotensis DC. 14735.

Triumfetta brevipes S. Watson. 9922.

Triumfetta columnaris Hochr. 4891, 9749, 10734, 12349, 17236, 17410bis. 
Triumfetta coriacea Hochr. 11056, 11193.

Triumfetta galeottiana Turcz. 1825, 1857, 2278, 4787, 5077, 5249, 6415, 6774, 6778, 6781, 6784, 6827, 9641, 9643, 9644, $9670,9720,10717,10718,10744,10832,11405,12484$, $12485,14731$.

Triumfetta goldmanii Rose. 1953, 3082, 4962, 5253, 6746 (Type of Triumfetta quercetorum Bullock), 6996, 9710, 9847, 10663, 10750, 12262, 12492, 14927.

Triumfetta heliocarpoides Bullock. 5159 (TYPUS), 6777, 6779, 6780, 7001.

Triumfetta lappula L. 15618, 15996.

Triumfetta paniculata Hook. \& Arn. 16118.

Triumfetta polyandra Sessé \& Moc. ex DC. 4591, 5300.

Triumfetta semitriloba Jacq. 1969, 2013, 3008, 4801, 4950, 6986, 7076, 7307, 8560, 11009, 16238, 24793.

Triumfetta simplicifolia (Sessé \& Moc.) Fryxell. 980, 3883, 6594, 7018 (Type of Triumfetta hintonii Sprague).

Waltheria indica L. 712, 1617, 2140, 2570, 2887, 4115, 4116, 5425, 5996, 9632, 15310, 23727.

Waltheria preslii Walp. 26499.

Waltheria pringlei Rose \& Standl. 2003, 4999, 6637, 13251.

Wissadula amplissima (L.) R.E. Fr. 2286, 2886, 4819, 11464 , 12452, 17615, 20575.

Wissadula periplocifolia (L.) C. Presl ex Thwaites. 6444, 6863.

\section{Family Marantaceae}

Calathea allouia (Aubl.) Lindl. 13896.

Calathea cyclophora Baker. 4282, 4295, 6747, 10260.

Maranta arundinacea L. 1155, 4428, 6465, 13971.

Thalia geniculata L. 9536.

Thalia geniculata L. 6596.

\section{Family Martyniaceae}

Martynia annua L. 1069, 1169, 4495, 6467, 6484, 6650, 6663, 10525, 10599, 14484, 20534.

Proboscidea louisiana (Mill.) Thell. 20598, 28846.

Proboscidea altheifolia (Benth.) Decne. 1202, 6661.

Proboscidea louisiana (Mill.) Thell. 19981, 21555, 24728, 25692, 28657.

\section{Family Melanthiaceae}

Anticlea frigida (Schltdl. \& Cham.) Zomlefer \& Judd. 2396, 4388, 4612, 8792, 13238, 15572.

Anticlea hintoniorum (B.L. Turner) Zomlefer \& Judd. 17150, 17200 (TYPUS), 17388, 17880, 18580, 18897, 19047, 20501, 21360, 22581, 26102, 26200, 26509.

Anticlea virescens (Kunth) Rydb. 5228, 15362.

Anticlea volcanica (Benth.) Baker. 8747, 10668.

Schoenocaulon ignigenum Frame. 17581.

Schoenocaulon intermedium Baker. 2690, 4970.

Schoenocaulon mortonii Brinker. 13465 (TYPUS).

Schoenocaulon officinale (Schltdl. \& Cham.) A. Gray ex Benth. 2039, 2420, 9397, 9506, 9539, 13226, 18302, 20318, 23064, 23283, 24870.

Schoenocaulon tenuifolium (M. Martens \& Galeotti) B.L. Rob. \& Greenm. 26116, 26123.
Schoenocaulon texanum Scheele. 17561, 18194, 18865, 20296, 20416, 21271, 21515, 22013, 22043, 23113, 23735 , 23846, 25379, 25513, 27873, 28728.

\section{Family Melastomataceae}

Arthrostemma alatum Triana. 12275, 15222.

Blakea calycularis (Naudin) Penneys \& Almeda. 14661, 14700.

Clidemia capitellata (Bonpl.) D. Don. 10781.

Clidemia hirta (L.) D. Don. 10768.

Clidemia submontana Rose ex Gleason. 15039.

Conostegia icosandra (Sw. ex Wikstr.) Urb. 14026.

Conostegia xalapensis (Bonpl.) D. Don ex DC. 442, 681, 7082, 7305, 9256, 10196.

Heterocentron mexicanum Hook. \& Arn. 11283.

Heterocentron mexicanum Hook. \& Arn. 6828, 7143, 8665, $8814,13513$.

Heterocentron subtriplinervium (Link \& Otto) A. Braun \& C.D. Bouché. 2351, 2665, 2926, 7035, 9849, 11065, 11339, $11631,12683$.

Leandra cornoides (Schltdl. \& Cham.) Cogn. 3799.

Leandra multiplinervis (Naudin) Cogn. 14510.

Miconia anisotricha (Schltdl.) Triana. 1377, 13933.

Miconia calvescens DC. 14332.

Miconia glaberrima (Schltdl.) Naudin. 14233, 14955, 17528bis.

Miconia hemenostigma Naudin. 7451.

Miconia heterothrix Gleason \& Wurdack. 9936 (TYPUS), 10752, 12710.

Miconia mexicana (Bonpl.) Naudin. 14188.

Miconia minutiflora (Bonpl.) DC. 14685.

Miconia mirabilis (Aubl.) L.O. Williams. 11185, 14717. Miconia obconica Gleason \& Wurdack. 10361 (TYPUS). Monochaetum calcaratum (DC.) Triana. 17480bis.

Monochaetum calcaratum (DC.) Triana. 2441, 6825, 7665, 8638, 12784, 13416, 13689, 15660.

Pterolepis trichotoma (Rottb.) Cogn. 2162, 8556, 10824, 11489, 12593, 14620.

Tibouchina congestiflora Todzia. 26337.

Tibouchina connata Gleason ex Todzia. 9952 (TYPUS).

Tibouchina durangensis Standl. 11070.

Tibouchina hintonii Gleason ex Todzia. 10612, 11015 (TYPUS), 11503, 14993.

Tibouchina latebracteolata Paul G. Wilson. 704, 2637, 7165 (TYPUS), 7175, 7175bis, 7181, 8695.

Tibouchina naudiniana (Decne.) Cogn. 12596, 12706.

Tibouchina scabriuscula (Schltdl.) Cogn. 110, 6815, 7066, 7177, 7183

Tibouchina tortuosa (Bonpl.) Almeda. 7231.

\section{Family Meliaceae}

Guarea glabra Vahl. 10097.

Melia azedarach L. 1446, 2298, 5976.

Swietenia humilis L. 3606, 4138, 6048, 14127, 15907.

Trichilia americana (Sessé \& Moc.) T.D. Penn. 737, 1279, 6442, 14535, 16303.

Trichilia havanensis Jacq. 3710, 7610, 8113.

Trichilia hirta L. 4427, 5576, 6097, 9263, 10073, 10618, 
11688, 12284, 13877, 13913, 14252.

Trichilia trifolia L. 14595, 15113.

\section{Family Menispermaceae}

Cissampelos pareira L. 3180, 3306, 4010, 4332, 5893, 14724.

Cocculus carolinus (L.) DC. 17812, 24208.

Cocculus diversifolius DC. 5811, 5833, 7556, 24287.

Disciphania mexicana Bullock. 4314 (TYPUS).

Hyperbaena ilicifolia Standl. 5562, 5842, 5843, 6927, 6970.

Menispermum canadense L. 7926.

\section{Family Menyanthaceae}

Nymphoides indica (L.) Kuntze. 1696, 4684, 7977, 15916.

\section{Family Molluginaceae}

Glinus radiatus (Ruiz \& Pav.) Rohrb. 947, 4065, 5447, 6001, 6066.

Mollugo verticillata L. 1471, 2467, 5519, 9144, 14544.

\section{Family Montiaceae}

Calandrinia ciliata (Ruiz \& Pav.) DC. 2115, 4911, 4923, 15341.

Claytonia perfoliata Donn ex Willd. 1709, 2738, 4620, 8398, 26113.

Phemeranthus aurantiacus (Engelm.) Kiger. 18988, 21098, 24357, 25362, 25659, 25697, 25843.

Phemeranthus parviflorus (Nutt.) Kiger. 18475.

\section{Family Moraceae}

Brosimum alicastrum Sw. 5661.

Dorstenia drakena L. 1298, 1464, 4940, 5078, 9172, 10590, 14354, 15119.

Ficus cotinifolia Kunth. 3136, 5735.

Ficus insipida Willd. 2984, 5808.

Ficus maxima Mill. 7309, 8888, 10083, 10275, 10276.

Ficus obtusifolia Kunth. 3644.

Ficus pertusa L. f. 9618, 11509.

Ficus petiolaris Kunth. 888, 3393, 7505.

Ficus retusa L. 3022.

Ficus velutina Humb. \& Bonpl. ex Willd. 3254, 3967, 6214.

Morus alba L. 3463, 3593.

Morus celtidifolia Kunth. 3462, 3613, 3627, 3843.

Trophis mexicana (Liebm.) Bureau. 10298.

Trophis racemosa (L.) Urb. 3165, 3181, 3186, 3643, 6750, 11540, 15901, 16291.

Family Moringaceae

Moringa oleifera Lam. 923, 3343, 9969.

Family Muntingiaceae

Muntingia calabura L. 3791, 5797, 9087, 11250, 14134, 15853.

Family Myricaceae

Morella cerifera (L.) Small. 3093, 4092, 5422, 6096, 7669.
Morella lindeniana (C. DC.) S. Knapp. 10730.

\section{Family Myrtaceae}

Calyptranthes hintonii Lundell. 4057, 6844, 7952 (TYPUS).

Eugenia alnifolia McVaugh. 16292 (TYPUS).

Eugenia axillaris (Sw.) Willd. 7679, 10076, 10146, 16244.

Eugenia capuli (Schltdl. \& Cham.) Hook. \& Arn. 10306, 13920, 14573, 14693.

Eugenia crenularis Lundell. 3778, 7695 (TYPUS).

Eugenia culminicola McVaugh. 8957 (TYPUS).

Eugenia guatemalensis Donn. Sm. 3368.

Eugenia hintonii Lundell. 9603 (TYPUS).

Eugenia michoacanensis Lundell. 13812 (TYPUS), 16212.

Eugenia salamensis Donn. Sm. 16124, 16266, 16274.

Eugenia yautepecana Lundell. 7172.

Myrcianthes fragrans (Sw.) McVaugh. 404, 2764, 3319, 3595, 5588.

Psidium guajava L. 5637, 6651, 7797, 24167.

Psidium guineense Sw. 772, 4383.

Psidium sartorianum (O. Berg) Nied. 3656, 5751, 12959.

\section{Family Namaceae}

Nama carnosa (Wooton) C.L. Hitchc. 29561.

Nama hispida A. Gray. 29560.

Nama stevensii C.L. Hitchc. 17322, 17837, 18181, 18225, 19526, 23883, 27002

Nama biflora Choisy. 16929, 17698, 17964, 22621, 22736, 24119, 24294, 24420, 25026, 25038, 25149, 25263, 25471.

Nama canescens C.L. Hitchc. 20644, 21584, 21729, 22312, 22346, 22571, 23036, 27650, 28741, 29168, 29238.

Nama carnosa (Wooton) C.L. Hitchc. 29268, 29327, 29377, 29408, 29419.

Nama dichotoma (Ruiz \& Pav.) Choisy. 19767, 20729, 21486, 22556, 23509, 25635, 26316, 27643.

Nama havardii A. Gray. 29282.

Nama hintoniorum G.L. Nesom. 20564 (TYPUS), 21240, 21408, 21521, 21665, 24646, 25838, 25846, 28112, 29064, 29197.

Nama hispida A. Gray. 16782, 18641, 19544, 19636, 21960, 24266, 27579, 28134, 28367, 28671, 28730, 28794, 29090.

Nama hitchcockii J.D. Bacon. 18084, 18345, 18785, 19430, 20950, 21732, 28709.

Nama jamaicensis L. 4604, 5465, 7583.

Nama johnstonii C.L. Hitchc. 23340, 27443, 28452, 28486.

Nama origanifolia Kunth. 11893, 17080.

Nama palmeri A. Gray ex Hemsl. 17659, 17909, 18168, 18974, 19809, 20177, 20371, 20596, 20817, 21184, 21786, $21855,21965,22371,23619,23858,24349,24465,25352$, 25361, 25507, 28183, 28891, 29094, 29099, 29203.

Nama prostrata Brand. 490, 615, 6081, 11968, 15097.

Nama quiexobrana J.D. Bacon \& J.A. McDonald. 26147, 26219, 26778, 26807.

Nama schaffneri A. Gray ex Hemsl. 16951.

Nama sericea Willd. ex Roem. \& Schult. 17643, 20525, 21764, 22559, 22898, 22939, 23102, 23473, 23492, 25043. Nama serpylloides A. Gray ex Hemsl. 28793.

Nama stenophylla A. Gray ex Hemsl. 28246, 28402, 28613, 
28629, 28771, 29434.

Nama undulata Kunth. 16777, 16778, 18915, 24215.

Wigandia urens (Ruiz \& Pav.) Kunth. 29805.

Wigandia urens (Ruiz \& Pav.) Kunth. 649, 2679, 5446, 10020, 11179, 11323, 13508, 13650, 15693.

\section{Family Nitrariaceae}

Peganum mexicanum A. Gray. 16794, 19429, 24834, 27606, 27674, 28371.

\section{Family Nyctaginaceae}

Acleisanthes acutifolia Standl. 29340.

Acleisanthes acutifolia Standl. 21958, 23535, 27444, 29357.

Acleisanthes angustifolia (Torr.) R.A. Levin. 24754, 28349. Acleisanthes lanceolata (Wooton) R.A. Levin. 29265.

Acleisanthes longiflora A. Gray. 16686, 20793, 22875, 23195, 23353, 25967, 28887, 29236.

Acleisanthes nana I.M. Johnst. 29392.

Acleisanthes obtusa (Choisy) Standl. 21533, 25869, 25875, 27750, 28722.

Acleisanthes palmeri (Hemsl.) R.A. Levin. 28401, 28620.

Acleisanthes purpusiana (Heimerl) R.A. Levin. 28417, 29608.

Acleisanthes undulata (B.A. Fowler \& B.L. Turner) R.A. Levin. 22866, 23345, 28512, 28831.

Allionia choisyi Standl. 24267, 24857, 28858.

Allionia incarnata L. 16691, 17524bis, 17524, 19952, 20783, 21477, 21526, 21768, 23133, 23182, 23543, 23618, 25520, 25655, 25680, 27732, 28374, 28433, 28510, 28670.

Anulocaulis eriosolenus (A. Gray) Standl. 23188, 23359, 23542, 23556, 27422, 28296, 28390, 28438, 28445, 28767 , 29318, 29615.

Anulocaulis hintoniorum B.L. Turner. 22693, 23192 (TYPUS).

Anulocaulis leiosolenus (Torr.) Standl. 29288.

Boerhavia anisophylla Torr. 16621, 20380, 22803, 23740, 23995, 24260, 24481, 24741, 24752, 25615, 25895, 27694, 28514, 28701.

Boerhavia coccinea Mill. 1213, 5095, 5539, 6115, 9168, 13044, 13370, 15065, 17613, 23722, 24988, 26502.

Boerhavia diffusa L. 9303.

Boerhavia erecta L. 1065, 2516, 4284, 5490, 5794, 9072, 9160, 10587, 12086, 17966, 26486, 28645.

Boerhavia gracillima Heimerl. 25978.

Boerhavia linearifolia A. Gray. 22040, 24499, 25775, 29379.

Boerhavia triquetra S. Watson. 27380.

Commicarpus praetermissus N.A. Harriman. 15911 (TYPUS).

Commicarpus scandens (L.) Standl. 15891, 16220, 26501. Cyphomeris crassifolia (Standl.) Standl. 19066, 20775, 20860, 21185, 22703, 23606, 23690, 23739, 24498, 25912, 28464.

Cyphomeris gypsophiloides (M. Martens \& Galeotti) Standl. 16898, 17658, 21564, 23117, 25693, 25701, 28132.

Mirabilis oxybaphoides (A. Gray) A. Gray. 18491.

Mirabilis albida (Walter) Heimerl. 17317, 19206, 21122,
$25447,25618,25678,25679,25682,25700,25727,27624$, 29159.

Mirabilis donahooiana Le Duc. 16017.

Mirabilis glabrifolia (Ortega) I.M. Johnst. 18498, 18511, 18572, 18749, 19011, 19735, 19753, 19780, 19908, 20417, $20487,22851,23006,23033,23132,23163,23383,23587$, $24403,24503,25158,25949,27045,27046,27246,27400$, 27448, 27590, 28720.

Mirabilis grandiflora (Standl.) Standl. 26480.

Mirabilis hintoniorum Le Duc. 13909 (TYPUS).

Mirabilis jalapa L. 1333, 4577, 4742, 19922.

Mirabilis linearis (Pursh) Heimerl. 18757.

Mirabilis longiflora L. 4412, 15970, 17603, 20491, 23107, 28577.

Mirabilis multiflora (Torr.) A. Gray. 18436, 18495, 19621, 25640, 27707, 27796.

Mirabilis nesomii B.L. Turner. 22319, 22351, 25499, 25567, 25714, 27290.

Mirabilis oxybaphoides (A. Gray) A. Gray. 17556, 17926, 19702, 25426, 25445, 25572, 25837, 28653, 28843, 29176. Mirabilis sanguinea Heimerl. 10297, 10452, 11976.

Nyctaginia capitata Choisy. 16683, 23378, 24282, 27751.

Okenia hypogaea Schltdl. \& Cham. 10323, 26495.

Okenia parviflora Paul G. Wilson. 6119, 6277, 10534, 12101 (TYPUS).

Pisonia aculeata L. 3382, 3412, 5521, 5652, 15884.

Pisoniella arborescens (Lag. \& Rodr.) Standl. 13262, 13391.

Salpianthus arenarius Bonpl. 10044, 10215, 12607, 12851, 13576, 15917.

Salpianthus macrodontus Standl. 2578, 2587, 5860.

Salpianthus purpurascens (Cav. ex Lag.) Hook. \& Arn. 2501, 2985, 11493.

Family Nymphaeaceae

Nymphaea ampla (Salisb.) DC. 6656, 15915.

Family Ochnaceae

Ouratea madrensis L. Riley. 15800, 15856.

\section{Family Oleaceae}

Forestiera angustifolia Torr. 18378, 20102, 22012, 27000, 27021, 27507, 28487.

Fraxinus berlandieriana A. DC. 1147, 12977.

Fraxinus cuspidata Torr. 16745, 17284, 17650, 18147, 20196, 24370, 24456, 24964, 24978.

Fraxinus greggii A. Gray. 16516, 18132, 18689, 21472, 25711, 25740.

Fraxinus uhdei (Wenz.) Lingelsh. 208, 303, 2415, 2903, 2947, 3844, 15414.

Jasminum mesnyi Hance. 25179.

Ligustrum lucidum Aiton. 252, 24721.

Menodora coulteri A. Gray. 16518, 18447, 21959, 23042, 24734, 25357, 25487, 25823, 27361, 27634, 27637, 28687, 28739.

Menodora chumleyi B.L. Turner. 20240, 22066 (TYPUS). Menodora geohintonii B.L. Turner. 21048 (TYPUS), 29467, 29471. 
Menodora gypsophila B.L. Turner. 24474, 25643 (TYPUS), 25836, 25863, 25897, 27198, 28727.

Menodora hintoniorum B.L. Turner. 19679 (TYPUS), 28583, 28649.

Menodora longiflora Engelm. ex A. Gray. 16550, 20770, 23851, 25648, 25864, 25870, 27767, 28338, 28700, 29443, 29463.

Menodora muellerae Rehder. 18220, 25349, 25810.

Menodora scabra A. Gray. 23533, 23539, 23571, 28308, 28373, 28439, 28517, 28763.

\section{Family Onagraceae}

Calylophus drummondianus Spach. 27718.

Calylophus hartwegii (Benth.) P.H. Raven. 16766, 17765, 18148, 18370, 18389, 18390, 19523, 19591, 20712, 21905, $22317,23054,23623,25459,25750,25965,28305,28468$, 28505, 29475.

Calylophus lavandulifolius (Torr. \& A. Gray) P.H. Raven. 23035, 25829, 29395.

Calylophus tubicula (A. Gray) P.H. Raven. 20467, 20758, 20952, 25784, 27041, 27197, 29396.

Epilobium angustifolium L. 18925.

Epilobium ciliatum Raf. 575, 794, 3291, 5763, 15649, 26050, 26187, 26747.

Fuchsia arborescens Sims. 8850, 11173, 14951, 15756, 17096, 17504bis.

Fuchsia fulgens DC. 823, 1144, 1744, 4311, 4894, 13952 , 13987, 15533.

Fuchsia lycioides Andrews. 316, 548, 634bis, 637, 845, 2825, 2846, 3055, 4044, 4942, 15006, 15452, 17156, 17210.

Fuchsia microphylla Kunth. 1902, 5149, 15729, 26173, 26286, 26425, 26508, 26592.

Fuchsia thymifolia Kunth. 620, 848, 1351, 1814, 1878, 4201, 4455, 4505.

Gaura boquillensis P.H. Raven \& Gregory. 29420, 29435.

Gaura calcicola P.H. Raven \& Gregory. 16637, 17772, 20385, 21164, 22090, 23575, 23997, 27580, 28474, 28488, 28624.

Gaura drummondii (Spach.) Torr. \& A. Gray. 21192.

Gongylocarpus rubricaulis Schltdl. \& Cham. 5039.

Lopezia miniata Lag. ex DC. 7106.

Lopezia racemosa Cav. 29775.

Lopezia hintonii R.C. Foster. 11075, 14902 (TYPUS).

Lopezia longiflora Decne. 3073, 3662, 3896.

Lopezia miniata Lag. ex DC. 11026, 11261, 12337, 13369, 16251.

Lopezia racemosa Cav. 1559, 2025, 2094, 2707, 6792, 6798, 6822, 9705, 11280, 13281, 15264, 15464, 19200, 20879, 22448, 22537, 26005, 26125, 26245, 26250, 26355, 26382.

Lopezia semeiandra Plitmann, P.H. Raven \& Breedlove. 12906, 13670, 15736, 15783.

Lopezia violacea Rose. 209, 5392.

Ludwigia adscendens (L.) H. Hara. 668, 4029, 5638, 12994, 13620, 13964.

Ludwigia erecta (L.) H. Hara. 1163, 3738.

Ludwigia hyssopifolia (G. Don) Exell. 9803.

Ludwigia octovalvis (Jacq.) P.H. Raven. 3558, 5103, 5551, 9207, 9832, 10029, 10565, 10603, 12529, 13599, 15038,
20535.

Ludwigia palustris (L.) Elliot. 5858.

Ludwigia peploides (Kunth) P.H. Raven. 3788, 9835, 11823.

Ludwigia peruviana (L.) H. Hara. 449, 1171, 1522, 1534, 5636.

Oenothera pubescens Willd. ex Spreng. 29743.

Oenothera tetraptera Cav. 29843.

Oenothera curtiflora W.L. Wagner \& Hoch. 16798, 20252, 20913.

Oenothera deserticola (Loes.) Munz. 7478.

Oenothera dissecta A. Gray ex S. Watson. 19506, 19577, 19590.

Oenothera epilobiifolia Kunth. 26165, 26538.

Oenothera hexandra (Ortega) W.L. Wagner \& Hoch. 617, 3876, 7688, 11863, 15643.

Oenothera jamesii Torr. \& A. Gray. 18776, 25877.

Oenothera kunthiana (Spach) Munz. 5607, 13590, 18733, 22019, 22667, 22733, 23073, 23264, 24560.

Oenothera laciniata Hill. 386, 387, 15014.

Oenothera macrosceles A. Gray. 17814, 21943, 27057, 29133, 29264.

Oenothera muelleri Munz. 17553, 27225.

Oenothera pennellii Munz. 18858, 20452, 29091.

Oenothera pubescens Willd. ex Spreng. 11947, 17585, 23230, 23966, 26697.

Oenothera rosea L'Hér. ex Aiton. 3727, 15032, 16832, 17143, 17546, 19406, 19422, 19549, 20558, 21200, 21822, 22000, 22100, 26550.

Oenothera speciosa Nutt. 18263, 18400, 21191, 24361, 24384, 25413, 25547.

Oenothera stubbei Dietrich, P.H. Raven \& W.L. Wagner. 17147, 20953, 21520, 25383.

Oenothera suffrutescens (Ser.) W.L. Wagner \& Hoch. 17144, 19416, 20937, 24395, 25427, 25916, 27627, 29166.

Oenothera tetraptera Cav. 18864, 20163, 20521, 20584, 21001, 21014, 24717, 29165.

Oenothera triloba Nutt. 27039, 27161.

\section{Family Opiliaceae}

Agonandra racemosa (DC.) Standl. 2423, 3124, 3308, 3312, 10081, 10243, 11774, 14023, 16304.

\section{Family Orchidaceae}

Acianthera hartwegiifolia (H. Wendl. \& Kraenzl.) R. Solano \& Soto Arenas. 2796, 3670.

Alamania punicea Lex. 859.

Anathallis scariosa (Lex.) Pridgeon \& M.W. Chase. 4235. Arpophyllum spicatum Lex. 2343, 8445, 11219.

Artorima erubescens (Lindl.) Dressler \& G.E. Pollard. 11175.

Aulosepalum nelsonii (Greenm.) Garay. 15835, 15900.

Aulosepalum pyramidale (Lindl.) M.A. Dix \& M.W. Dix. 478, 537, 3674.

Barkeria obovata (Presl) Christenson. 3649.

Barkeria naevosa (Lindl.) Schltr. 5566, 5583, 7390.

Barkeria obovata (Presl) Christenson. 3138, 3371, 11724.

Barkeria scandens (Lex.) Dressler \& Halb. 9732. 
Barkeria uniflora (Lex.) Dressler \& Halb. 2557, 2964, 3318, 5050, 5128, 9768, 11011, 11155, 11214, 11468, 12500, 16053.

Bletia gracilis Lodd. 9396, 15551.

Bletia adenocarpa Rchb. f. 1288, 1505, 11360, 13186, 17235, 18480.

Bletia campanulata Lex. 13464.

Bletia coccinea Lex. 1299, 1507, 4694, 9131, 14416.

Bletia concolor Dressler. 10382, 10481 (TYPUS).

Bletia gracilis Lodd. 1118, 4379, 9734, 14400, 15988.

Bletia macristhmochila Greenm. 775, 891, 7718, 15401.

Bletia parkinsonii Hook. 19333.

Bletia punctata Lex. 1119, 9477.

Bletia purpurata A. Rich. \& Galeotti. 1599, 9653.

Bletia purpurea (Lam.) DC. 3222, 3563, 7090, 7504, 15796.

Bletia reflexa Lindl. 1074, 1133, 1188, 1204, 2445, 2757, 3070, 8664, 9739, 9759, 14383, 15657.

Bulbophyllum cirrhosum L.O. Williams. 14191 (TYPUS).

Campylocentrum schiedei (Rchb. f.) Benth. ex Hemsl. 10284.

Catasetum integerrimum Hook. 8242.

Clowesia rosea Lindl. 16162.

Clowesia rosea Lindl. 12669, 12699, 16008.

Corallorhiza bulbosa A. Rich. \& Galeotti. 25557.

Corallorhiza maculata (Raf.) Raf. 15092.

Corallorhiza odontorhiza (Willd.) Poir. 22674.

Corallorhiza maculata (Raf.) Raf. 14436, 26837.

Corallorhiza odontorhiza (Willd.) Poir. 12814.

Corallorhiza wisteriana Conrad. 17393.

Cranichis subumbellata A. Rich. \& Galeotti. 2345, 5242, 20900.

Cranichis subumbellata A. Rich. \& Galeotti. 2771, 9881, 11200, 11208.

Cuitlauzina egertonii (Lindl.) Dressler \& N.H. Williams. 14175.

Cuitlauzina pendula Lex. 3821, 3904, 7686, 11942, 13715, 13727.

Cyclopogon pringlei (S. Watson) Soto Arenas. 13848.

Cypripedium irapeanum Lex. 1186, 1208, 1434, 4438, 9230, 14420.

Cyrtopodium macrobulbon (Lex.) G.A. Romero-Gonzalez \& Carnevali. 14260, 15928.

Deiregyne eriophora (B.L. Rob. \& Greenm.) Garay. 564, 3692, 9024, 14218, 21830, 22618, 25171, 26561.

Deiregyne pseudopyramidalis (L.O. Williams) Garay. 7653.

Dichaea squarrosa Lindl. 900.

Dichaea squarrosa Lindl. 15426, 17073.

Dichaea trichocarpa (Sw.) Lindl. 596.

Dichromanthus cinnabarinus (Lex.) Garay. 18013.

Dichromanthus aurantiacus (Lex.) Salazar \& Soto Arenas. 1240, 7980, 9254, 14425, 17227, 17417, 26735, 27218.

Dichromanthus cinnabarinus (Lex.) Garay. 19618, 19766, 20482, 20721, 21377, 22179, 22353, 22938, 24319, 25758, 27686, 29209.

Dichromanthus michuacanus (Lex.) Salazar \& Soto Arenas. 3017, 5291, 13484, 14773.
Encyclia pollardiana (Withner) Dressler \& G.E. Pollard. 13757bis.

Encyclia adenocarpa (Lex.) Schltr. 3828, 3909, 6015, 14122.

Encyclia adenocaula (Lex.) Schltr. 644, 3823, 11881, 14261.

Encyclia meliosma (Rchb. f.) Schltr. 741, 991, 3783.

Encyclia microbulbon (Hook.) Schltr. 418, 3669, 13714, 14073, 14177.

Encyclia oestlundii (Ames, F.T. Hubbard \& C. Schweinf.) Hágsater \& Stermitz. 10089.

Encyclia pollardiana (Withner) Dressler \& G.E. Pollard. 13851 (TYPUS), 13928.

Encyclia selligera (Lindl.) Schltr. 14138, 14139.

Epidendrum cardiophorum Schltr. 14641.

Epidendrum cilioccidentale Hágsater \& L. Sánchez. 13782, 14258.

Epidendrum citrosmum Hágsater. 10740, 11799.

Epidendrum matudae L.O. Williams. 5897.

Epidendrum anisatum Lex. 383.

Epidendrum arbuscula Lindl. 11220.

Epidendrum ciliare L. 7800, 10259, 10289, 12509bis.

Epidendrum clowesii Bateman ex Lindl. 11769.

Epidendrum eximium L.O. Williams. 5604.

Epidendrum juergensenii Rchb. f. 472, 3505, 7721.

Epidendrum magnoliae Muhl. 24648.

Epidendrum polyanthum Lindl. 13648.

Epidendrum vandifolium Lindl. 13677.

Epipactis gigantea Douglas ex Hook. 18255, 19134, 22036.

Erycina echinata (Kunth) Lindl. 15798, 15875.

Erycina hyalinobulbon (Lex.) N.H. Williams \& M.W. Chase. 8919, 17511bis.

Funkiella parasitica (A. Rich. \& Galeotti) Salazar \& Sto Arenas. 18794, 22826.

Funkiella hyemalis (A. Rich. \& Galeotti) Schltr. 14799, 15727, 26112, 26140, 26209, 26290.

Funkiella minutiflora (A. Rich. \& Galeotti) Salazar \& Soto Arenas. 22636 (Type of Galeottiella hintoniorum Todzia).

Galeoglossum tubulosum (Lind1.) Salazar. 3264, 22689.

Galeoglossum tubulosum (Lindl.) Salazar. 3691, 8834, 8963, 26413.

Galeottiella sarcoglossa (A. Rich. \& Galeotti) Schltr. 14790, 15361.

Gongora galeottiana A. Rich. \& Galeotti. 13939.

Goodyera oblongifolia Raf. 18341.

Goodyera striata Rchb. f. 22509.

Govenia purpusii Schltr. 22957.

Govenia lagenophora Lindl. 1104, 4451, 8282.

Govenia liliacea (Lex.) Lindl. 14430, 15086, 21285, 22133, 22961, 23081, 23220, 25470, 26754, 26768.

Govenia superba (Lex.) Lindl. ex Lodd. 6160, 13026, 13098, 14423, 14431.

Guarianthe aurantiaca (Bateman ex Lindl.) Dressler \& W.E. Higgins. 2969, 3010, 11154, 11213, 11768, 11791, 13645, 16270, 16296.

Habenaria agapitae R. González \& Reynoso. 15122.

Habenaria calicis R. González. 26760. 
Habenaria clypeata Lindl. 1733.

Habenaria cuevasiana R. González \& Cuevas-Figueroa. 15102.

Habenaria distans Griseb. 16002.

Habenaria filifera S. Watson. 1454, 15138.

Habenaria nogeirana R. González \& Cuevas-Figueroa. 26952.

Habenaria rzedowskiana R. González. 1692.

Habenaria tamazulensis R. González \& Cuev.-Fig. 12273.

Habenaria clypeata Lindl. 1578, 1735, 4528, 4640, 9270, 9295, 14518, 15126, 17412bis.

Habenaria diffusa A. Rich. \& Galeotti. 2756.

Habenaria distans Griseb. 14517.

Habenaria guadalajarana S. Watson. 1391, 1506, 9264, 9268, 22202.

Habenaria jaliscana S. Watson. 15135.

Habenaria novemfida Lindl. 15357.

Habenaria quinqueseta (Michx.) Eaton. 14479.

Habenaria strictissima Rchb. f. 11418, 15208, 26770, 26838.

Habenaria trifida Kunth. 1607, 4526, 9269, 9455, 15519.

Hexalectris nitida L.O. Williams. 18767, 29175.

Hexalectris brevicaulis L.O. Williams. 9393, 12181 (TYPUS).

Hexalectris grandiflora (A. Rich. \& Galeotti) L.O. Williams. 22944, 25453.

Hintonella mexicana Ames. 283, 8926, 8928 (TYPUS), 8951, 17506bis.

Homalopetalum pachyphyllum (L.O. Williams) Dressler. 1691 (TYPUS).

Isochilus langlassei Schltr. 14281.

Isochilus linearis (Jacq.) R. Br. 899, 4605, 13678.

Kraenzlinella hintonii (L.O. Williams) R. Solano. 14174 (TYPUS).

Laelia albida Bateman ex Lindl. 9915.

Laelia autumnalis (Lex.) Lindl. 2434, 2997, 13467, 15656.

Laelia rubescens Lindl. 9798, 11601, 12551, 16176, 16227 , $16236,16237$.

Leochilus carinatus (Knowles \& Westc.) Lindl. 1128, 1375, 8060.

Leochilus oncidioides Knowles \& Westc. 12668.

Lepanthes nagelii Salazar \& Soto Arenas. 970, 1858.

Liparis vexillifera (Lex.) Cogn. 1400, 4701, 8286.

Lockhartia oerstedii Rchb. f. 14366.

Lycaste crinita Lindl. 10101, 13741, 14176.

Macroclinium lexarzanum (Hágsater \& R. González) Dodson. 13728

Malaxis alvaroi García-Cruz, R. Jiménez \& L. Sánchez. 23205, 23233, 23266.

Malaxis brachystachys (Rchb. f.) Kuntze. 23302, 27985.

Malaxis carnosa (Kunth) C. Schweinf. 17226.

Malaxis corymbosa (S. Watson) Kuntze. 29184.

Malaxis crispata (Lindl.) R. González. 26673, 26682, 26741.

Malaxis chica Todzia. 29208.

Malaxis fastigiata (Rchb. f.) Kuntze. 23219.

Malaxis abieticola Salazar \& Soto Arenas. 23244.

Malaxis brachystachys (Rchb. f.) Kuntze. 19167, 20745,
21621, 22513, 23083, 23310, 23311, 23427, 23450, 25437. Malaxis chica Todzia. 23259 (TYPUS).

Malaxis fastigiata (Rchb. f.) Kuntze. 9222, 21493, 23175, 24324, 24333, 26763, 26767.

Malaxis histionantha (Link, Klotsch \& Otto) Garay \& Dunst. 10581.

Malaxis lepidota (Finet) Ames. 10684.

Malaxis macrostachya (Lex.) O. Kuntze. 1707, 14470, 23232, 23274, 23275, 26060, 26784, 26813.

Malaxis maianthemifolia Schltdl. \& Cham. 17168.

Malaxis unifolia Michx. 1800.

Maxillaria variabilis Bateman ex Lindl. 3648.

Maxillaria cucullata Lindl. 14513, 15105, 17172.

Maxillaria mexicana J.T. Atwood. 10554 (TYPUS).

Maxillaria rhombea Lindl. 1328, 14362.

Maxillaria variabilis Bateman ex Lindl. 3890, 4370, 13945 , 14710.

Meiracyllium gemma Rchb. f. 12478, 14920.

Mesadenus chiangii (M.C. Johnst.) Garay. 21565, 23384, 23871, 25502, 28554.

Mesadenus polyanthus (Rchb. f.) Schltr. 20174, 20299, 25201, 27086.

Mormodes oestlundiana Salazar \& Hágsater. 15790.

Myrmecophila galeottiana (A. Rich.) Rolfe. 16164.

Notylia barkeri Lindl. 16126.

Oestlundia ligulata (Lex.) Soto Arenas. 14171.

Oestlundia cyanocolumna (Ames, F.T. Hubb. \& C. Schweinf.) W.E. Higgins. 22634.

Oestlundia ligulata (Lex.) Soto Arenas. 7722, 9016, 10087, 10105, 15924.

Oncidium geertianum C. Morren. 13438.

Oncidium microstigma Rchb. f. 13377.

Oncidium unguiculatum Lindl. 236.

Oncidium brachyandrum Lindl. 253, 3376, 3572, 3708, 14093.

Oncidium ghiesbreghtianum A. Rich. \& Galeotti. 13743.

Oncidium hastatum (Bateman) Lindl. 676, 3705, 3763, 3933, 9028, 13725, 13756.

Oncidium hintonii L.O. Williams. 13784 (TYPUS).

Oncidium mexicanum (L.O. Williams) M.W. Chase \& N.H. Williams. 14640.

Oncidium reflexum Lindl. 2209, 2519, 2533, 2535, 2549, 2621, 2656, 2968, 2970, 9383, 9580, 16186.

Oncidium reichenheimii (Linden \& Rchb. f.) Garay \& Stacy. 3903, 3906, 9026.

Oncidium stelligerum Rchb. f. 13716.

Oncidium tigrinum Lex. 12389, 12436.

Oncidium unguiculatum Lindl. 2776, 8929, 11168.

Platanthera brevifolia (Greene) Kraenzl. 27981.

Platanthera brevifolia (Greene) Kraenzl. 4914.

Platanthera limosa Lindl. 4619, 14507, 15356.

Ponera exilis Dressler. 2346 (TYPUS).

Ponera juncifolia Lindl. 2369.

Ponthieva ephippium Rchb. f. 22540.

Ponthieva mexicana (A. Rich. \& Galeotti) Salazar. 22469, 23488, 23507, 24800.

Ponthieva schaffneri (Rchb. f.) E.W. Greenw. 23451, 23524, 27497. 
Ponthieva ephippium Rchb. f. 23222.

Ponthieva mexicana (A. Rich. \& Galeotti) Salazar. 14880. Ponthieva schaffneri (Rchb. f.) E.W. Greenw. 19260, 21283, 22450, 22536, 23487, 24869, 24913, 27271.

Prosthechea squalida (Lex.) Soto Arenas \& Salazar. 13644, 13723.

Prosthechea trulla (Rchb. f.) W.E. Higgins. 14136, 14259. Prosthechea bicamerata (Rchb. f.) W.E. Higgins. 3697.

Prosthechea cochleata (L.) W.E. Higgins. 10250, 15047, 15227.

Prosthechea concolor (Lex.) W.E. Higgins. 10104, 14172, 15923.

Prosthechea chacaoensis (Rchb. f.) W.E. Higgins. 10095.

Prosthechea chondylobulbon (A. Rich. \& Galeotti) W.E. Higgins. 1868.

Prosthechea hastata (Lindl.) W.E. Higgins. 14301.

Prosthechea micropus (Rchb. f.) W.E. Higgins. 3570, 6202, 10106, 14198.

Prosthechea michuacana (Lex.) W.E. Higgins. 419, 3709, 3771, 10147, 13742.

Prosthechea pastoris (Lex.) Espejo \& López-Ferrari. 13988.

Prosthechea pterocarpa (Lindl.) W.E. Higgins. 889, 14916, 15427.

Prosthechea squalida (Lex.) Soto Arenas \& Salazar. 374, 3507, 3610, 3661, 11871, 13758, 14249.

Prosthechea varicosa (Bateman ex Lindl.) W.E. Higgins. 2830, 9029, 14315, 26435, 26613.

Rhyncholaelia glauca (Lindl.) Schltr. 13699.

Rhynchostele candidula (Rchb. f.) Soto Arenas \& Salazar. 26608.

Rhynchostele cervantesii (Lex.) Soto Arenas \& Salazar. 26370.

Rhynchostele maculata (Lex.) Soto Arenas \& Salazar. 11210, 13705.

Rhynchostele aptera (Lex.) Soto Arenas \& Salazar. 592, 5919, 14240, 14316.

Rhynchostele cervantesii (Lex.) Soto Arenas \& Salazar. 278, 391, 3300, 3658, 8980, 11143, 13698, 15150, 15750.

Rhynchostele galeottiana (A. Rich.) Soto Arenas \& Salazar. 13712, 13713.

Rhynchostele maculata (Lex.) Soto Arenas \& Salazar. 2754, 10180 (Type of Brassia oestlundiana L.O. Williams).

Rhynchostele rossii (Lindl.) Soto Arenas \& Salazar. 9027.

Rossioglossum insleayi (Baker ex Lindl.) Garay \& G.C.

Kenn. 11209, 13567, 15409.

Sacoila lanceolata (Aubl.) Garay. 10108, 13846.

Sarcoglottis schaffneri (Rchb. f.) Ames. 26930.

Sarcoglottis schaffneri (Rchb. f.) Ames. 22811, 22917, 25287.

Scaphyglottis sessilis (Rchb. f.) Foldats. 3663, 7313.

Schiedeella durangensis (Ames \& C. Schweinf.) Burns-Bal. 18166.

Schiedeella llaveana (Lindl.) Schltr. 9009.

Schiedeella nagelii (L.O. Williams) Garay. 27867.

Schiedeella durangensis (Ames \& C. Schweinf.) Burns-Bal. 20289, 21894, 22778, 24055, 25233.

Schiedeella llaveana (Lindl.) Schltr. 7619.
Sobralia galeottiana A. Rich. 4173.

Spiranthes graminea Lindl. 626, 6161.

Stelis greenwoodii Soto Arenas \& R. Solano. 12674.

Stelis rubens Schltr. 14709.

Stelis villosa (Knowles \& Westc.) Pridgeon \& M.W. Chase. 4642.

Stelis emarginata (Lindl.) Soto Arenas \& R. Solano. 15683.

Stelis quadrifida (Lex.) R. Solano \& Soto Arenas. 354, 3650, 13643.

Stelis retusa (Lex.) Pridgeon \& M.W. Chase. 6812.

Stelis villosa (Knowles \& Westc.) Pridgeon \& M.W. Chase. 969, 14514, 15018, 16070.

Tamayorkis ehrenbergii (Rchb. f.) R. González \& Szlach. 1322, 14467, 23208, 23265, 26679.

Tamayorkis hintonii (Todzia) R. González \& Szlach. 25438.

Tamayorkis wendtii (Salazar) R. González \& Szlach. 25934.

Tamayorkis hintonii (Todzia) R. González \& Szlach. 21284 (TYPUS).

Trichocentrum carthagenense (Jacq.) M.W. Chase \& N.H. Williams. 13646.

Trichocentrum cebolleta (Jacq.) M.W. Chase \& N.H. Williams. 3205, 3362, 11241, 11793, 13647.

Trichocentrum lindenii (Brogn.) M.W. Chase \& N.H. Williams. 13807.

Trichocentrum pachyphyllum (Hook.) R. Jiménez \& Carnevali. 296, 510, 3540, 13842, 15905.

Vanilla pompona Schiede. 13815, 15910.

\section{Family Orobanchaceae}

Agalinis gypsophila B.L. Turner. 29886.

Agalinis gypsophila B.L. Turner. 18090, 18328, 18770, 21501, 27017, 27894, 28912.

Agalinis peduncularis (Benth.) Pennell. 2120, 2323, 2337, 5328.

Aureolaria greggii (S. Watson) Pennell. 18462.

Aureolaria greggii (S. Watson) Pennell. 18254, 19694, 20609, 25496, 27010, 27462, 27716.

Buchnera obliqua Benth. 18017, 29703.

Buchnera pusilla Kunth. 12874, 12922, 13740, 14886, 14944, 15734, 15775.

Buchnera retrorsa Philcox. 2127 (TYPUS).

Buchnera tenuissima Philcox. 5275 (TYPUS).

Castilleja lanata A. Gray. 29873, 29874.

Castilleja scorzonerifolia Kunth. 29871.

Castilleja arvensis Schltdl. \& Cham. 15563.

Castilleja aurea B.L. Rob. \& Greenm. 1736, 2353, 3054, 4975, 9380, 9558, 11103, 15260.

Castilleja bella Standl. 17012, 17049, 17109, 20998.

Castilleja chiapensis Brandegee. 11080.

Castilleja falcata Eastw. 1030.

Castilleja galehintoniae G.L. Nesom. 18149, 18422 (TYPUS), 18449, 18530, 20933, 21914, 28277.

Castilleja gracilis Benth. 16052.

Castilleja integrifolia L. f. 9939, 19020, 20743, 21265, 26071, 26221, 26225, 26306, 26387, 26668, 26737, 26774, 
27375, 28714.

Castilleja lanata A. Gray. 16659, 19443, 19587, 19605, 20089, 20251, 21079, 21155, 22340, 23032, 23371, 23701, 25862, 25953, 27463, 27551, 28363, 28400, 28448, 28623, 28667, 28742, 28805, 28839, 29366, 29413, 29587.

Castilleja mexicana (Hemsl.) A. Gray. 18258, 18448, 19698, 19745, 20119, 24488, 25374, 27652, 28918, 29136.

Castilleja rigida Eastw. 19688, 20100, 20301, 20714, 20726, 20967, 21063, 22338, 22339, 23540, 25759, 25772, 27005.

Castilleja scorzonerifolia Kunth. 1720, 2668, 3682, 8312, 12927, 13015, 13588, 17019, 18235, 19306, 20156, 20273, 20313, 20386, 20427, 20778, 20903, 21044, 21341, 21516, 21726, 21758, 21767, 21859, 21929, 22406, 23153, 23317, 23621, 23700, 24239, 25431, 25604, 26130, 26136, 27926, 27966, 28242, 28260.

Castilleja tenuiflora Benth. 207, 7139, 11099, 11286, 11674, $12463,12985,16302,16655,17440,17791,17921,17949$, 18574, 19193, 19824, 19894, 20144, 20293, 21015, 21061, $21179,21725,21986,22249,22409,24233,24372,24446$, 24864, 26351, 26452, 27340, 27415, 27540, 29169.

Castilleja tenuifolia M. Martens \& Galeotti. 1515, 2553, 2722, 8296, 8298, 9473, 11094, 11415, 12214, 13423, 13533, 15577, 17429 bis, 17991.

Castilleja tolucensis Kunth. 430.

Castilleja zempoaltepetlensis G.L. Nesom. 26133, 26451, 26581, 26800.

Conopholis alpina Liebm. 3301, 3845, 7444, 10161, 14115, 17583, 18798, 20185, 22614, 22918, 25234.

Escobedia grandiflora (L. f.) Kuntze. 2074, 4867.

Escobedia laevis Schltdl. \& Cham. 18037.

Lamourouxia dasyantha (Cham. \& Schltdl.) W.R. Ernst. 29791.

Lamourouxia multifida Kunth. 29729, 29771.

Lamourouxia dasyantha (Cham. \& Schltdl.) W.R. Ernst. 17452, 18103, 23609, 28963, 29006.

Lamourouxia macrantha M. Martens \& Galeotti. 26755.

Lamourouxia multifida Kunth. 149, 2044, 3287, 11109, 11288, 13278, 17428bis, 26830.

Lamourouxia paneroi B.L. Turner. 26101, 26236, 26393.

Lamourouxia rhinanthifolia Kunth. 4864, 21298, 21350, 22129, 22927, 25666.

Lamourouxia viscosa Kunth. 196, 2696, 2791, 2847, 12946 , 25052.

Lamourouxia xalapensis Kunth. 2372, 2828, 5151, 8639.

Melasma physalodes (D. Don) Melch. 2202, 2359, 3049, 5409, 8887, 9779, 11051, 11556, 13336.

Orobanche ludoviciana Nutt. 16656, 21789, 25498, 27020.

Orobanche multicaulis Brandegee. 19494, 27545.

Pedicularis canadensis L. 17381, 19462, 22211, 22743, 22913, 25194.

Pedicularis hintonii McVaugh \& Mellich. 14789 (TYPUS). Pedicularis mexicana Zucc. ex Benth. 956, 1715, 8343, 8441, 13169.

Pedicularis tripinnata M. Martens \& Galeotti. 965, 4218, 6166.

Seymeria deflexa Eastw. 25930.

Seymeria gypsophila B.L. Turner. 23753.

Seymeria laciniata (M. Martens \& Galeotti) Standl. 14908.
Seymeria scabra A. Gray. 17653, 24671. Seymeria tamaulipana B.L. Turner. 17706, 24815, 24900. Seymeria virgata (Kunth) Benth. ex DC. 18323, 18742, 19016, 20419, 21331, 23489, 25709.

\section{Family Oxalidaceae}

Oxalis microcarpa Benth. 10359.

Oxalis alpina (Rose) Knuth. 4904, 7936, 15094, 15345, 29201, 29261.

Oxalis corniculata L. 622, 3398, 4185, 13631, 16941, 18283, 19415, 19910, 20347, 20939, 21808, 21823, 24020, 25850, 27576.

Oxalis decaphylla Kunth. 21033.

Oxalis dimidiata Donn. Sm. 4335.

Oxalis drummondii A. Gray. 21049, 21132, 22106, 22240, 22810, 22863, 23331, 23365, 23646, 24507.

Oxalis frutescens L. 4574, 9094, 14596.

Oxalis hernandezii DC. 7937, 13840, 15027, 15076, 15077, 15082.

Oxalis jacquiniana Kunth. 974.

Oxalis lasiandra Zucc. 4169.

Oxalis latifolia Kunth. 657, 1002, 7809, 15002, 15083, 16823, 17547, 18236, 20256, 20472, 21002, 22238, 22949, 23078, 24241, 26249.

Oxalis lunulata Zucc. 26593.

Oxalis madrensis S. Watson. 22598.

Oxalis rhombifolia Jacq. 1469, 3976, 4143, 4572, 6490bis, 11875 bis.

Oxalis stricta L. 15001.

Oxalis tetraphylla Cav. 549, 883, 15029, 15104, 15110.

\section{Family Papaveraceae}

Argemone platyceras Link \& Otto. 29748.

Argemone albiflora Hornem. 19157, 19290, 19472, 20130, 22925, 23099, 24974, 27256, 27392, 28868.

Argemone echinata G.B. Ownbey. 17676, 17822, 18502, 19449, 19579, 20397, 20942, 20969, 21509, 21802, 27558. Argemone fruticosa Thurb. ex A. Gray. 16582, 29245, 29303, 29440.

Argemone grandiflora Sweet. 27710, 27951.

Argemone intermedia Sweet. 20768, 25219.

Argemone mexicana L. 284, 3432, 3715, 3827, 7283, 7355, 7520, 9999, 10014, 11789, 12963, 17794, 18146, 20357, 21123, 21188, 21488, 21844, 25217, 28151.

Argemone ochroleuca Sweet. 17523, 17555, 19099, 20055, 24007.

Argemone platyceras Link \& Otto. 4930, 6575, 16600, 17549, 17759, 18060, 26683, 27450.

Argemone subalpina J.A. McDonald. 17383, 17550.

Argemone turnerae A.M. Powell. 19156.

Bocconia arborea S. Watson. 1000, 1526, 10466, 11763, 16179.

Bocconia frutescens L. 5358, 6730, 7671, 10156, 25212.

Bocconia hintoniorum B.L. Turner. 26227 (TYPUS), 26623.

Bocconia latisepala S. Watson. 17945, 28193.

Corydalis pseudomicrantha Fedde. 16753, 17985, 18159, 18812, 20286, 22601, 26572. 
Fumaria parviflora Lam. 3495, 3849.

Hunnemannia fumariifolia Sweet. 16624, 17450, 19276, 19316, 20120, 21806, 22759, 24140.

Hunnemannia hintoniorum G.L. Nesom. 21876 (TYPUS), 25226, 25384, 28589.

\section{Family Passifloraceae}

Passiflora adenopoda DC. 12264.

Passiflora biflora Lam. 3781, 7405.

Passiflora colimensis Mast. \& Rose. 1151, 1210, 4340, 8013, 8018, 8043, 9095, 9141, 10925, 13094.

Passiflora coriacea Juss. 3030, 4655.

Passiflora exsudans Zucc. 819, 3848, 4187, 4889, 8000, 8224, 17405 bis, 22256.

Passiflora filipes Benth. 11218.

Passiflora foetida L. 992, 3659, 4411, 5514, 6879, 8045, 8076, 13049, 17782, 17947, 21602, 26498.

Passiflora holosericea L. 13809.

Passiflora jorullensis Kunth. 1794, 2139, 6558, 6809, 7009, 7088, 7834, 12260.

Passiflora membranacea Benth. 14241, 26333, 26375, 26617.

Passiflora mexicana Juss. 1949, 3652, 5718, 6926, 11007.

Passiflora pavonis Mast. 816, 5931, 7974, 17081.

Passiflora pilosa Ruiz \& Pav. ex DC. 4261, 8069.

Passiflora podadenia Killip. 15230.

Passiflora porphyretica Mast. 2936, 3569, 5752, 11563, 12184, 13593, 15802.

Passiflora suberosa L. 4519, 4700, 6533, 8607, 13492.

Passiflora subpeltata Ortega. 470, 655, 3863, 4160, 7638, 7913, 8233, 13051.

Passiflora uncinata J.M. MacDougal. 10749 (TYPUS).

Passiflora viridiflora Cav. 10999.

Turnera callosa Urb. 744.

Turnera diffusa Willd. ex Schult. 13081, 19288, 20797, 22860, 24253, 27819, 28340.

Turnera pumilea L. 8451.

Turnera ulmifoia L. 1066, 1363, 6509, 9189, 9212, 13070, 15056.

Turnera ulmifolia L. 1012, 1875, 10558.

Family Pedaliaceae

Sesamum indicum L. 10524.

\section{Family Pentaphylacaceae}

Cleyera integrifolia (Benth.) Choisy. 116, 282, 1099, 3835, 3886, 7192, 7227, 7704, 7990, 11950, 15085, 15098, 15374, 17098.

Symplococarpon purpusii (Brandegee) Kobuski. 2426, 3081 (Type of Symplococarpon airy-shawianum Kobuski), 3678, 8653 (Type of Eurya hintonii Bullock).

Ternstroemia lineata DC. 281, 310, 646, 3728, 5606, 5914, 10152, 11949, 14070, 14289, 15753.

Ternstroemia sylvatica Schltdl. \& Cham. 14328, 14408.

Family Phrymaceae

Erythranthe dentiloba (B.L. Rob. \& Fernald) G.L. Nesom. 15349.
Erythranthe glabrata (Kunth) G.L. Nesom. 678, 17804, 25853, 26186, 26536.

Erythranthe pallens (Greene) G.L. Nesom. 29028.

Hemichaena spinulosa (S. Watson) Thieret. 19191, 19204, 25690, 27083, 28710.

Leucocarpus perfoliatus (Kunth) Benth. 14979.

\section{Family Phyllanthaceae}

Margaritaria nobilis L. f. 9555, 10264, 12285, 15167.

Phyllanthus acuminatus Vahl. 14351, 14606, 15965, 16109.

Phyllanthus caroliniensis Walter. 20559.

Phyllanthus coalcomanensis Croizat. 15857 (TYPUS), 15859, 15967.

Phyllanthus galeottianus Baill. 1285, 4497, 10482.

Phyllanthus graveolens Kunth. 4333, 5330, 13882.

Phyllanthus neoleonensis Croizat. 16907.

Phyllanthus niruri L. 8601, 10549, 10970.

Phyllanthus petaloideus Paul G. Wilson. 2376 (TYPUS).

Phyllanthus polygonoides Nutt. ex Spreng. 4148, 16891, 23849, 25634, 25971, 27991, 28184.

Phyllanthus purpusii Brandegee. 587, 8956.

\section{Family Phyllonomaceae}

Phyllonoma laticuspis (Turcz.) Engl. 3091, 3667, 4105, 5395, 10164, 14114, 14186, 14283, 14327.

\section{Family Phytolaccaceae}

Agdestis clematidea Moc. \& Sessé ex DC. 25023.

Petiveria alliacea L. 4760, 5294, 6660, 10850, 11607, 12300, 15118.

Phytolacca icosandra L. 3860, 5336, 9240, 11450, 12200, 12852, 16183, 17503, 21615, 24115, 24408, 28975.

Phytolacca rivinoides Kunth \& C.D. Bouché. 10760.

Phytolacca rugosa A. Braun \& C.D. Bouché. 11082, 11896, 26349.

Rivina humilis L. 1667, 4372, 6560, 7757, 10994, 13891, 14525, 15204, 16004, 16311, 16847, 17617, 19227, 20798, 21214, 21412, 21997, 23877, 24174, 28191.

Trichostigma octandrum (L.) H. Walter. 3655, 3925, 5909, 14154.

\section{Family Picramniaceae}

Alvaradoa amorphoides Liebm. 1916, 2508, 2543, 5736, 6964, 6965, 7057, 12560, 12696, 13661, 16035, 16115.

Picramnia antidesma Sw. 5236, 7387, 10206, 10279, 14133, 14163.

Picramnia polyantha (Benth.) Planch. 14211.

\section{Family Piperaceae}

Peperomia asarifolia Schltdl. \& Cham. 10652, 11346.

Peperomia berlandieri Miq. 21302, 21613, 22138.

Peperomia blanda (Jacq.) Kunth. 17644, 17671, 20854, 23124, 24651, 25040.

Peperomia bracteata A.W. Hill. 1556.

Peperomia claytonioides Kunth. 23077.

Peperomia galioides Kunth. 1877, 2033, 12416.

Peperomia gracillima S. Watson. 6634, 9145. 
Peperomia hintonii Yunck. 4648 (TYPUS).

Peperomia hispidula (Sw.) A. Dietr. 2370, 4651, 6529, 14751, 14780.

Peperomia lanceolatopeltata C. DC. 2196, 2539, 5130.

Peperomia serpens (Sw.) Loudon. 10280.

Peperomia tetraphylla (G. Forst.) Hook \& Arn. 2798, 3963, 7226.

Piper novogalicianum Bornstein. 4287 (Type of Arctottonia tejupilcoana Trel.).

Piper xanthostachyum C. DC. 14984.

Piper aduncum L. 3400, 10348, 10576, 14152, 14687.

Piper amalago L. 414, 708, 1053, 3143, 3671, 24510.

Piper arboreum Aubl. 12034.

Piper auritum Kunth. 6170, 14017, 17828, 23815.

Piper colotlipanense Bornstein. 10261, 10266.

Piper chinantlense M. Martens \& Galeotti. 11049.

Piper glabrescens (Miq.) C. DC. 14050.

Piper hispidum Sw. 415, 3672.

Piper leucophyllum (Miq.) C. DC. 3103, 14829.

Piper marginatum Jacq. 16044.

Piper michelianum C. DC. 13836.

Piper stipulaceum Opiz. 11372, 13855.

Piper tuberculatum Jacq. 16015.

Piper uhdei C. DC. 10506.

Piper umbellatum L. 709, 1379, 4407, 10777, 14644.

Piper unguiculatum Ruiz \& Pav. 14054.

\section{Family Plantaginaceae}

Bacopa monnieri (L.) Wettst. 5555, 5868, 13598, 14522, 14589, 15805, 29231.

Bacopa repens (Sw.) Wettst. 4682, 5492.

Bacopa rotundifolia (Michx.) Wettst. 9403, 13203, 14559.

Bacopa salzmannii (Benth.) Wetts. ex Edwall. 5199.

Callitriche deflexa A. Braun ex Hegelm. 4988.

Callitriche heterophylla Pursh. 8920.

Cymbalaria muralis P. Gaertn., B. Mey. \& Scherb. 2848, 3625, 3626, 3864.

Mabrya erecta (Hemsl.) Elisens. 23377, 23563, 28414, 28470, 28610, 28787, 29427, 29438.

Maurandya antirrhiniflora Humb. \& Bonpl. ex Willd. 19211, 20172, 20603, 23041, 25644, 27595, 28656, 28738, 29617.

Maurandya barclayana Lindl. 16930, 17184, 22164, 22260, 22349, 22370, 23468, 24490, 25589.

Maurandya erubescens (D. Don) A. Gray. 4588, 4866.

Maurandya scandens (Cav.) Pers. 14863.

Mecardonia procumbens (Mill.) Small. 29813.

Mecardonia procumbens (Mill.) Small. 1140, 2583, 4631, $5730,6855,10413,10715,11899,12860,12993,15036$, 17663, 17780, 18479, 20122, 20377, 25350.

Penstemon roseus (Sweet) G. Don. 18009.

Penstemon amphorellae Crosswh. 18473, 19743, 22358.

Penstemon baccharifolius Hook. 25797.

Penstemon barbatus (Cav.) Roth. 15351, 17104, 17357, 17870, 17979, 18878, 20212, 20285, 20431, 22432, 22975, 23164, 24396, 25405, 27930, 28177, 29015.

Penstemon campanulatus (Cav.) Willd. 148, 268, 872, 2113, 2130, 2902, 5061, 8004, 13284, 13569, 15522, 17055, 17877 , 18565, 18888, 18898, 20989, 21012, 21353, 22126, 22281,
23146, 27277.

Penstemon galloensis G.L. Nesom. 19166 (TYPUS), 21296, 22107, 22215, 23916, 24577.

Penstemon gentianoides (Kunth) Poir. 1032, 3282, 3990, 8250, 13236, 26134, 26801.

Penstemon havardii A. Gray. 18424, 18496, 18653, 28650. Penstemon hintonii Straw. 9751, 10709, 11290 (TYPUS).

Penstemon imberbis (Kunth) Trautv. 2106, 2379, 8300, 11348, 15262.

Penstemon isophyllus B.L. Rob. 26713.

Penstemon lanceolatus Benth. 16548, 19726, 20391, 20413, 21892, 23118, 23171, 23582, 23698, 25964, 28104, 29011, 29183, 29460.

Penstemon leonensis Straw. 17385, 17579, 17879, 20512, 20744, 22579.

Penstemon luteus G.L. Nesom. 21009 (TYPUS).

Penstemon miniatus Lindl. 12348, 15471, 17418bis.

Penstemon roseus (Sweet) G. Don. 12381, 13235, 15277, 26299, 26729.

Plantago australis Lam. 852, 15937, 20334, 20358, 20359, 22458, 24016.

Plantago floccosa Decne. 21273, 21630.

Plantago lanceolata L. 16698, 19407, 24381, 25404, 26999.

Plantago major L. 5627, 5809, 19439, 21574, 22089, 23267 , 26547, 26711, 26840.

Plantago nivea Kunth. 1305, 1553, 6258.

Plantago patagonica Jacq. 18438, 18985.

Plantago rhodosperma Decne. 21866, 21915, 21934, 27157, 27596, 29089, 29097.

Plantago tolucensis Pilg. 433.

Rhodochiton hintonii (Elisens) D.A. Sutton. 14465 (TYPUS).

Russelia cuneata B.L. Rob. 9188, 11393, 11585, 16057, 16129.

Russelia hintonii Lundell. 10921 (TYPUS).

Russelia jaliscensis B.L. Rob. 10465.

Russelia leptopoda Lundell. 13845 (TYPUS).

Russelia polyedra Zucc. 11105, 12892, 13197.

Russelia retrorsa Greene. 15620, 17472bis.

Russelia syringifolia Schldtl. \& Cham. 25211.

Russelia tenuis Lundell. 15841 (TYPUS).

Russelia teres Lundell. 2112, 16127 (TYPUS).

Russelia ternifolia Kunth. 141, 1167, 2111, 3191, 7297, 27103, 28559.

Scoparia dulcis L. 4558, 5506, 12863.

Schistophragma pusillum Benth. 1448, 5558, 9498, 12023, 15242.

Sibthorpia rotundifolia (Ruiz \& Pav.) Edwin. 1717, 4069, 6410, 21699, 22748, 23458, 23964, 24088.

Stemodia coahuilensis (Henrickson) B.L. Turner. 28644, 28646.

Stemodia durantifolia (L.) Sw. 2531, 5296, 5437, 5484, 5873, 12027, 12636, 12992.

Stemodia macrantha B.L. Rob. 3325, 7382, 9877, 11265 , 11691, 12924, 12958.

Stemodia verticillata (Mill.) Hassl. 2966.

Veronica americana (Raf.) Schwein. ex Benth. 1043, 4203. 
Veronica peregrina L. 2104, 2864, 3536, 4632, 5769, 5890.

Veronica persica Poir. 18155, 18927, 22648.

Veronica polita R.E. Fr. 2102, 8341.

\section{Family Platanaceae}

Platanus mexicana Moric. 17591, 17649.

Platanus occidentalis L. 22185, 25140.

Platanus rzedowskii Nixon \& J.M. Poole. 17514, 21543, 22987, 24096, 24183, 25216, 25239.

\section{Family Plumbaginaceae}

Plumbago pulchella Boiss. 7982, 13155, 17725, 19777, 21121, 21211, 22599, 28199.

Plumbago zeylanica L. 2648, 6905, 9560, 10986, 11502, 11659, 12589, 12898, 13337, 14861, 15317, 16185, 17627, 25013,28198 .

\section{Family Poaceae}

Achnatherum eminens (Cav.) Barkworth. 19742, 19902, 27431, 27451.

Achnatherum multinode (Scribn. ex Beal) Valdés-Reyna \& Barkworth. 20083.

Achnatherum robustum (Vasey) Barkworth. 19361, 20982, 27536.

Agrostis ghiesbreghtii E. Fourn. 2734, 2742, 5590, 8393.

Agrostis schaffneri E. Fourn. 2739.

Agrostis tolucensis Kunth. 1713, 4933.

Amelichloa clandestina (Hack.) Arriaga \& Barkworth. 16759, 17563, 18214, 19132, 19408.

Andropogon angustatus (J. Presl) Steud. 2189, 2632, 4995, 5299, 6668, 9684.

Andropogon glomeratus (Walter) Britton, Stearn \& Pogg. 19963, 22379.

Andropogon leucostachyus Kunth. 9461.

Andropogon liebmannii Hack. 1413.

Andropogon virginicus L. 23290.

Anthaenantia lanata (Kunth) Benth. 4587.

Anthephora hermaphrodita (L.) Kuntze. 3947, 9285, 9816.

Aristida ternipes Cav. 6673.

Aristida capillacea Lam. 2634.

Aristida divaricata Humb. \& Bonpl. ex Willd. 19746.

Aristida gibbosa (Nees) Kunth. 2275, 9593.

Aristida hintonii Hitchc. 1729 (TYPUS), 1873, 2169, 6567, 6571, 9389.

Aristida pansa Wooton \& Standl. 23010.

Aristida purpurea Nutt. 27311, 27454, 27471, 28380, 28625.

Aristida ternipes Cav. 6682, 9809, 11410.

Arundinella hispida (Humb. \& Bonpl. ex Willd.) Kuntze. 5303.

Arundinella berteroniana (Schult.) Hitchc. \& Chase. 25305, 25322.

Arundinella deppeana Nees ex Steud. 3162, 3184, 11629.

Arundinella hispida (Humb. \& Bonpl. ex Willd.) Kuntze.

2414, 2584, 3041, 5097, 5312.

Arundo donax L. 5712, 6478.

Axonopus arsenei Swallen. 1574.
Axonopus compressus (Sw.) P. Beauv. 6532.

Blepharidachne bigelovii (S. Watson) Hack. 23003.

Bothriochloa barbinodis (Lag.) Herter. 2065, 19889, 21503.

Bothriochloa hirtifolia (J. Presl) Henrard. 2283, 2357, 5352.

Bothriochloa hybrida (Gould) Gould. 20827.

Bothriochloa laguroides (DC.) Herter. 19885, 27526.

Bothriochloa longipaniculata (Gould) Allred \& Gould. 2870, 3782, 4947.

Bouteloua barbata Lag. 6439, 23029, 28668, 28811.

Bouteloua breviseta Vasey. 23189, 27418, 27420, 27452 , 29263.

Bouteloua curtipendula (Michx.) Torr. 2254, 4011, 6669, 9616, 15238, 19681, 20920, 21405, 24496, 27307, 27445, 27477.

Bouteloua chasei Swallen. 19644, 20756, 21532, 28092, 28449.

Bouteloua dactyloides (Nutt.) J.T. Columbus. 18964, 19418, 22380.

Bouteloua dimorpha J.T. Columbus. 1746, 1761, 5462, 6446, 6632, 6648, 12030, 13304, 15192.

Bouteloua disticha (Kunth) Benth. 6871.

Bouteloua erecta (Vasey \& Hack.) J.T. Columbus. 4782, 6477, 12092.

Bouteloua gracilis (Kunth) Lag. ex Griffiths. 16844, 18006, 19861, 21720, 27304, 27306, 27727, 27892.

Bouteloua hirsuta Lag. 9332bis, 9501.

Bouteloua johnstonii Swallen. 29353.

Bouteloua juncea (Desv. ex P. Beauv.) Hitchc. 2512, 5463, 5981, 6882.

Bouteloua multifida (Griffiths) J.T. Columbus. 2645, 9496bis, 9499

Bouteloua polymorpha (E. Fourn.) J.T. Columbus. 4231, 4272, 4297, 9050 .

Bouteloua radicosa (E. Fourn.) Griffiths. 13221, 21379, 21432.

Bouteloua repens (Kunth) Scribn. \& Merr. 1792, 1854, 2190, 2291, 5980, 6653, 6672, 9500, 24439.

Bouteloua scorpioides Lag. 19740, 27887.

Bouteloua simplex Lag. 27314.

Bouteloua uniflora Vasey. 19588, 21070, 25510, 27453, 27889, 27902.

Brachypodium mexicanum (Roem. \& Schult.) Link. 27932. Brachypodium pringlei Scribn. ex Beal. 21089, 23143.

Bromus anomalus Rupr. ex E. Fourn. 1337, 2702.

Bromus carinatus Hook. \& Arn. 4186, 4984, 20284, 22422, 23145, 23158.

Bromus ciliatus L. 2459, 8395.

Calamagrostis orizabae (Rupr. ex E. Fourn.) Beal. 4622.

Cenchrus ciliaris L. 23352, 24732, 27432.

Cenchrus clandestinus (Hochst. ex Chiov.) Morrone. 29701, 29781.

Cenchrus echinatus L. 1160, 4800, 12066.

Cenchrus michoacanus H.F. Gut. \& Morrone. 2268, 6794, 9794.

Cenchrus multiflorus J. Presl. 12075.

Cenchrus myosuroides Kunth. 16866. 
Cenchrus pilosus Kunth. 2930, 2980, 5112, 5556, 9631, 13313.

Cenchrus polystachios (L.) Morrone. 1159, 2191, 4013, 5262, 9824.

Cenchrus prolificus (Chase) Morrone. 3166, 3229, 14030.

Cenchrus spinifex Cav. 20612, 23689.

Cinna poiformis (Kunth) Scribn. \& Merr. 1301, 1712.

Cortaderia selloana (Schult. \& Schult. f.) Asch. \& Graebn. 24832.

Cottea pappophoroides Kunth. 28495.

Cynodon dactylon (L.) Pers. 1625, 3624, 3745, 5194, 5456, 5667, 5760, 9833, 27047.

Chaetium bromoides (J. Presl) Benth. ex Hemsl. 2418, 5043, 6748.

Chascolytrum subaristatum (Lam.) Desv. 3531, 21632.

Chloris rufescens Lag. 1439, 9321.

Chloris submutica Kunth. 19554.

Chloris virgata Sw. 1646, 1760, 4765, 6030, 9279, 15163, 28816.

Chusquea galeottiana Rupr. ex Munro. 14271.

Chusquea nelsonii Scribn. \& J.G. Sm. 14779.

Dactylis glomerata L. 19131.

Dactyloctenium aegyptium (L.) Willd. 1632, 5585, 5993, 9194, 9281, 14662, 15202.

Dichanthelium acuminatum (Sw.) Gould \& C.A. Clark. 22081.

Dichanthelium commutatum (Schult.) Gould. 2527 (Type of Panicum hintonii Swallen).

Diectomis fastigiata (Sw.) P. Beauv. 5161, 6609, 6674.

Digitaria ciliaris (Retz.) Koeler. 1628, 2260, 4691.

Digitaria argillacea (Hitchc. \& Chase) Fernald. 1628bis, 4490, 4990bis.

Digitaria badia (Scribn. \& Merr.) Fernald. 6517.

Digitaria californica (Benth.) Henrard. 16581.

Digitaria leucites (Trin.) Henrard. 2108, 4983.

Dinebra aquatica (Scribn. \& Merr.) P.M. Peterson \& N. Snow. 6675.

Dinebra panicea (Retz.) P.M. Peterson \& N. Snow. 5486, 6033, 6462, 6680, 9363.

Dinebra panicoides (J. Presl) P.M. Peterson \& N. Snow. 6043, 6427, 6676.

Diplachne fusca (L.) Kunth. 5543.

Disakisperma dubium (Kunth) P.M. Peterson \& N. Snow. 19556, 19639, 27293, 27298.

Distichlis littoralis (Engelm.) H.L. Bell \& J.T. Columbus. 21982, 28317, 28326, 28747.

Echinochloa colona (L.) Link. 5975, 9306, 9322.

Echinochloa crus-galli (L.) P. Beauv. 4443, 4474.

Echinochloa crus-pavonis (Kunth) Schult. 1177, 1616, 3302, 3303, 4221, 4345, 5517, 12195.

Eleusine indica (L.) Gaertn. 1395, 1546, 1753, 2304, 3866, 5193, 5680, 9364.

Elionurus ciliaris Kunth. 2270, 2352, 6756.

Elionurus tripsacoides Humb. \& Bonpl. ex Willd. 5229, 12168.

Enneapogon desvauxii P. Beauv. 23639, 27430, 27473, 28669, 28775, 28883, 29291.

Eragrostis mexicana (Hornem.) Link. 5153.
Eragrostis cilianensis (All.) Vignolo ex Janch. 5990, 15161.

Eragrostis ciliaris (L.) R. Br. 2197, 2244, 2548, 2646, 5617.

Eragrostis intermedia Hitchc. 21275.

Eragrostis japonica (Thunb.) Trin. 2565.

Eragrostis lugens Nees. 1442, 3537.

Eragrostis maypurensis (Kunth) Steud. 2192, 9817.

Eragrostis mexicana (Hornem.) Link. 1639, 2767, 2854, 5019, 5114, 19555, 19572, 24972.

Eragrostis palmeri S. Watson. 27459.

Eragrostis pectinacea (Michx.) Nees. 4211, 5535, 6633, 6704.

Eragrostis pilosa (L.) P. Beauv. 4716.

Eragrostis unioloides (Retz.) Nees ex Steud. 2614.

Eriochloa acuminata (J. Presl) Kunth. 6367.

Eriochloa nelsonii Scribn. \& J.G. Sm. 1941, 2727, 5171, 6753, 6943, 12141, 13326, 15195.

Eriochloa punctata (L.) Desv. ex Ham. 5648, 5671, 6007.

Erioneuron avenaceum (Kunth) Tateoka. 19617, 19866, 23004, 25649, 27446, 27708, 27780, 27890, 27903.

Erioneuron pilosum (Buckley) Nash. 19739.

Euclasta condylotricha (Hochst. ex Steud.) Stapf. 2609, 5015, 6710 .

Festuca amplissima Rupr. 2453, 8791, 14954, 21344, 22302, 23440.

Festuca hintoniana E.B. Alexeev. 17243 (TYPUS).

Festuca perennis (L.) Columbus \& J.P. Sm. 2336, 2836.

Festuca pringlei St.-Yves. 19142.

Festuca valdesii M. González-Ledezma \& S.D. Koch. 20278 (TYPUS).

Glyceria striata (Lam.) Hitchc. 3875, 6075.

Hemarthria altissima (Poir.) Stapf \& C.E. Hubb. 6031.

Hesperostipa neomexicana (Thurb.) Barkworth. 19741.

Heteropogon contortus (L.) P. Beauv. ex Roem. \& Schult. 1821, 2068, 5306, 6700, 9400, 23572, 23772, 24006, 24753, 24770, 28515.

Heteropogon melanocarpus (Elliott) Benth. 6608.

Hilaria belangeri (Steud.) Nash. 12029, 12093.

Hilaria cenchroides Kunth. 4733, 6707, 13113, 15213.

Hilaria ciliata (Scribn.) Nash. 9310.

Hilaria hintonii Sohns. 4502 (TYPUS), 6437.

Hopia obtusa (Kunth) Zuloaga \& Morrone. 19575, 27571.

Hordeum jubatum L. 19425.

Hymenachne amplexicaulis (Rudge) Nees. 5814, 6975, 14697.

Hyperthelia dissoluta (Nees ex Steud.) Clayton. 1638, 5056, 9690.

Ichnanthus nemorosus (Sw.) Döll. 14718.

Imperata brasiliensis Trin. 13271.

Ixophorus unisetus (J. Presl) Schltdl. 1161, 1972, 3737, 3739 , 5994, 6441, 12065, 13219.

Jarava ichu Ruiz \& Pav. 1718, 2744, 29825.

Koeleria macrantha (Ledeb.) Schult. 20505.

Lasiacis divaricata (L.) Hitchc. 14932.

Lasiacis procerrima (Hack.) Hitchc. 1948, 2408, 14690.

Lasiacis rhizophora (E. Fourn.) Hitchc. 10809, 14633.

Lasiacis ruscifolia (Kunth) Hitchc. 1767, 4847, 9386, 11569, 
16121.

Lasiacis sorghoidea (Desv. ex Ham.) Hitchc. \& Chase. 1909, 4958, 6722, 9581, 11381, 14610, 15156.

Leersia hexandra Sw. 2503.

Leptochloa pluriflora (E. Fourn.) P. M. Peterson \& N. Snow. 23882.

Leptochloa virgata (L.) P. Beauv. 5495, 5633.

Megathyrsus maximus (Jacq.) B.K. Simon \& S.W.L. Jacobs. 4823.

Melica montezumae Piper. 22784.

Melinis repens (Willd.) Zizka. 17623, 19964, 20020, 23989, 24435, 24990.

Metcalfia mexicana (Scribn.) Conert. 27810.

Microchloa kunthii Desv. 1514, 4476, 6381.

Mnesithea granularis (L.) de Koning \& Sosef. 1748, 4766, 4844, 15198.

Morronea parviglumis (Hack.) Zuloaga \& Scataglini. 5066. Morronea trichidiachnis (Döll) Zuloaga \& Scataglini. 7426.

Muhlenbergia gigantea (E. Fourn.) Hitchc. 9635.

Muhlenbergia arenacea (Buckley) Hitchc. 28660.

Muhlenbergia cenchroides (Humb. \& Bonpl. ex Willd.) P.M. Peterson. 1566, 1891, 3490, 4977, 8394, 9746, 15352.

Muhlenbergia ciliata (Kunth) Trin. 2307, 2625, 5231.

Muhlenbergia distichophylla (J. Presl) Kunth. 11635.

Muhlenbergia diversiglumis Trin. 1912, 2093, 2306, 2522, 5277, 6726, 6817.

Muhlenbergia dubia E. Fourn. 21508, 24809, 27773, 27809.

Muhlenbergia dumosa Scribn. ex Vasey. 3226, 7135.

Muhlenbergia emersleyi Vasey. 2081, 2324, 21337, 25135, 25302.

Muhlenbergia eriophylla Swallen. 5307 (TYPUS).

Muhlenbergia flavida Vasey. 1565.

Muhlenbergia geminiflora (Kunth) P.M. Peterson. 1586, 1830, 2210, 2302, 2325, 4721, 6770, 13183.

Muhlenbergia gigantea (E. Fourn.) Hitchc. 2386, 2626, 5302, 12471.

Muhlenbergia gypsophila Reeder \& C. Reeder. 19868, 21531, 23597, 23636, 23647, 23655, 23999, 27496, 27891, 28080.

Muhlenbergia hintonii Swallen. 3059 (TYPUS).

Muhlenbergia implicata (Kunth) Trin. 2079, 2163, 2262, 2341, 2641, 6068, 6816, 9614.

Muhlenbergia jaime-hintonii P.M. Peterson \& Valdés-Reyna. 22698, 23707, 23766, 27725, 28234.

Muhlenbergia macroura (Kunth) Hitchc. 1899, 2309, 2910, 13495, 20506, 20868.

Muhlenbergia minutissima (Steud.) Swallen. 2455, 15340.

Muhlenbergia pereilema P.M. Peterson. 1911, 1998, 2060, 2681, 3076, 5062, 6802, 9687.

Muhlenbergia peruviana (P. Beauv.) Steud. 2447, 4931, 8406.

Muhlenbergia phalaroides (Kunth) P.M. Peterson. 1855, 4932.

Muhlenbergia phleoides (Kunth) J.T. Columbus. 19863, 27303, 27309.

Muhlenbergia plumbea (Trin.) Hitchc. 1721.

Muhlenbergia plumiseta J.T. Columbus. 1967, 4788, 4976,
5071, 5261.

Muhlenbergia pubigluma Swallen. 23654, 27299, 27980.

Muhlenbergia quadridentata (Kunth) Trin. 4389, 4935, 8404.

Muhlenbergia ramulosa (Kunth) Swallen. 2311, 2318, 2437, 5073, 8397.

Muhlenbergia repens (J. Presl) Hitchc. 27292.

Muhlenbergia rigida (Kunth) Kunth. 1567, 1681, 2682, 4720, 19887, 20631, 27308, 27310, 27922.

Muhlenbergia robusta (E. Fourn.) Hitchc. 1959, 2053, 2591, 13409, 22553.

Muhlenbergia scoparia Vasey. 12699bis.

Muhlenbergia setifolia Vasey. 27335.

Muhlenbergia spiciformis Trin. 20835, 20859, 28109.

Muhlenbergia stricta (J. Presl) Kunth. 1853, 2083.

Muhlenbergia tenella (Kunth) Trin. 1962, 2052, 2361, 2566, 4974, 5118, 6942, 9621.

Muhlenbergia tenuifolia (Kunth) Kunth. 19793, 19888, 27808.

Muhlenbergia torreyi (Kunth) Hitchc. ex Bush. 27026, 27073.

Muhlenbergia tricholepis (Torr.) J.T. Columbus. 4922, 23968.

Muhlenbergia utilis (Torr.) Hitchc. 2319, 4944, 8399.

Muhlenbergia versicolor Swallen. 2719.

Muhlenbergia villiflora Hitchc. 18789, 19532, 27044, 27296, 28673.

Muhlenbergia virescens (Kunth) Trin. 14803, 27883, 27886, 27945.

Muhlenbergia virletii (E. Fourn.) Soderstr. 3219, 17498bis. Munroa pulchella (Kunth) L.D. Amarilla. 19561, 22331, 23007, 23554, 27043, 27426, 28377, 28882.

Nassella mucronata (Kunth) R.W. Pohl. 1890, 19301bis, 20184, 20194, 20946, 27495.

Nassella tenuissima (Trin.) Barkworth. 16789, 19640.

Ocellochloa biglandulare (Scribn. \& J.G. Sm.) Zuloaga \& Morrone. 14646, 14725.

Ocellochloa pulchella (Raddi) Zuloaga \& Morrone. 10801. Olyra latifolia L. 16137.

Oplismenus burmannii (Retz.) P. Beauv. 1882bis, 1947, 2567, 3001, 4948, 7046, 9619, 9695, 15295.

Oplismenus compositus (L.) P. Beauv. 1882, 1952, 2051, 2237, 2366, 2490, 5265.

Oplismenus hirtellus (L.) P. Beauv. 21204, 24897.

Oryza sativa L. 7799.

Panicum aztecanum Zuloaga \& Morrone. 2010 (TYPUS).

Panicum ghiesbreghtii E. Fourn. 1428, 4413.

Panicum hirticaule J. Presl. 4442, 6337, 6423, 9284.

Panicum parcum Hitchc. \& Chase. 1996, 2000, 6686.

Panicum pilosum Sw. 14683.

Panicum plenum Hitchc. \& Chase. 9440.

Panicum stramineum Hitchc. \& Chase. 1062.

Panicum trichoides Sw. 2002, 2153, 2249, 2642, 6690, 9376, 14688.

Pappophorum bicolor E. Fourn. 28251.

Pappophorum mucronulatum Nees. 27434.

Paspalum botterii (E. Fourn.) Chase. 4515, 5206.

Paspalum conjugatum P.J. Bergius. 2294, 4510, 6008, 9304, 
10807, 14647.

Paspalum cymbiforme E. Fourn. 2532.

Paspalum denticulatum Trin. 1426, 1659, 4363, 6029, 21952, 23134.

Paspalum distichum L. 4418.

Paspalum hintonii Chase. 1807 (TYPUS).

Paspalum humboldtianum Flüggé. 1232, 1440, 1623, 1804, 2521, 4710, 4809, 6067, 11400, 20052.

Paspalum longicuspe Nash. 6657.

Paspalum multicaule Poir. 5162.

Paspalum notatum Alain ex Flüggé. 1585, 1657, 9245, 13106, 15154.

Paspalum paniculatum L. 1443, 3944, 5233, 9433, 9434, 14659.

Paspalum plenum Chase. 1608, 1743, 4017, 6181, 9554.

Paspalum plicatulum Michx. 4005, 9282.

Paspalum prostratum Scribn. \& Merr. 2393, 4858.

Paspalum pubiflorum Rupr. ex Fourn. 20910.

Paspalum squamulatum E. Fourn. 4493.

Paspalum tumidum Kuhlm. 1974, 5017, 6711.

Paspalum virgatum L. 1161bis, 4522, 12148bis.

Peyritschia deyeuxioides (Kunth) Finot. 1558, 2317, 2380,

2684, 2777, 3220, 4989, 5594.

Pharus latifolius L. 10846.

Pharus mezii Prodoehl. 16043, 16059.

Phleum alpinum L. 17295.

Piptochaetium fimbriatum (Kunth) Hitchc. 27391, 27416, 28895.

Piptochaetium virescens (Kunth) Parodi. 1336, 1893, 2315.

Pleuraphis mutica Buckley. 28379.

Poa annua L. 1527, 2101, 5348, 27711.

Poa mulleri Swallen. 17253.

Poa nemoralis L. 3996.

Polypogon elongatus Kunth. 838, 1329, 2806, 3530, 3634, 6219.

Polypogon interruptus Kunth. 2313.

Polypogon viridis (Gouan) Breistr. 3457, 3521, 5600, 5768, 6221, 21709, 22084, 24390.

Pseudechinolaena polystachya (Kunth) Stapf. 9891.

Sacciolepis myuros (Lam.) Chase. 8654.

Saccharum officinarum L. 2993.

Sarga trichoclada (Rupr. ex Hack.) Spangler. 2252, 9692, 10829, 12178.

Scleropogon brevifolius Phil. 20757, 27042.

Schizachyrium brevifolium (Sw.) Nees ex Büse. 2326, 5034, 5035bis.

Schizachyrium condensatum (Kunth) Nees. 2413.

Schizachyrium malacostachyum (J. Presl) Nash. 5052.

Schizachyrium scoparium (Michx.) Nash. 27899, 27914, 27929, 27944.

Schizachyrium tenerum Nees. 2070.

Setaria grisebachii E. Fourn. 4344, 17520, 17685, 20851, 28180.

Setaria leucopila (Scribn. \& Merr.) K. Schum. 20567.

Setaria liebmannii E. Fourn. 1214, 6308, 6698, 9071, 12048 , 12053, 12069, 12070, 12088.

Setaria longipila E. Fourn. 2726, 5365.

Setaria macrostachya Kunth. 10566, 17520, 19774.
Setaria parviflora (Poir.) Kerguélen. 1318, 4012, 4291, 4481, 5635, 9320, 14372, 14667, 20912, 27398.

Setaria pumila (Poir.) Roem. \& Schult. 19886, 19962, 28176.

Setariopsis auriculata (E. Fourn.) Scribn. 6464, 9286, 9309.

Setariopsis latiglumis (Vasey) Scribn. 5016, 6720, 6780bis. Sorghastrum incompletum (J. Presl) Nash. 1970, 2069, 2348, 2492, 2493, 2563, 2938, 5033, 5160, 9685, 9686, 9688, 9700 .

Sorghastrum nutans (L.) Nash. 23771.

Sorghum bicolor (L.) Moench. 1783, 4839.

Sorghum halepense (L.) Pers. 1427, 4031, 5457, 5552, 13140, 13270, 16865, 20911, 24212, 27525.

Sporobolus airoides (Torr.) Torr. 19553, 23031, 27291, 27682, 27880.

Sporobolus cryptandrus (Torr.) A. Gray. 19571.

Sporobolus hintonii T.G. Hartley. 2610 (TYPUS).

Sporobolus indicus (L.) R. Br. 20908.

Sporobolus junceus (P. Beauv.) Kunth. 1429, 2388, 9750, 9925, 12417.

Sporobolus macrospermus Scribn. ex Beal. 1798, 1876, 1934, 2124, 9608.

Sporobolus spiciformis Swallen. 27295, 27297.

Sporobolus trichodes Hitchc. 9331, 9430.

Steinchisma cupreum (Hitchc. \& Chase) W.V. Br. 4414.

Steinchisma laxum (Sw.) Zuloaga. 1649, 4006, 4479.

Trachypogon spicatus (L. f.) Kuntze. 2236, 2321, 4709, 6701, 9507, 14639, 15468.

Tragus berteronianus Schult. 21468.

Tridens muticus (Torr.) Nash. 28378.

Triniochloa micrantha (Scribn.) Hitchc. 3092, 5230, 5313.

Triniochloa stipoides (Kunth) Hitchc. 2444, 24810.

Tripsacum dactyloides (L.) L. 1444, 1609, 2183, 6755, 9384, 15206, 20569.

Tripsacum pilosum Scribn. \& Merr. 2179.

Trisetum irazuense (Kuntze) Hitchc. 2314.

Trisetum spicatum (L.) K. Richt. 17242, 22431.

Trisetum viride (Kunth) Kunth. 2908.

Trisetum virletii E. Fourn. 2460, 2745, 3248, 3625bis, 5592, 15747, 17531bis.

Tristachya avenacea (J. Presl) Scribn. \& Merr. 2793.

Urochloa arizonica (Scribn. \& Merr.) Morrone \& Zuloaga. 6285.

Urochloa fusca (Sw.) B.F. Hansen \& Wunderlin. 5205, 6009, 6309, 6460.

Urochloa mollis (Sw.) Morrone \& Zuloaga. 3942, 9283.

Urochloa mutica (Forssk.) T.Q. Nguyen. 5168, 6880.

Urochloa reptans (L.) Stapf. 6487.

Vulpia myuros (L.) C.C. Gmel. 1722, 2119, 3522, 8405.

Zea mays L. 1758.

Zeugites americanus Willd. 2387, 2883, 3446, 5248, 5595, 14739, 14783, 14950.

Zeugites hintonii T.G. Hartley. 4538 (TYPUS).

Zeugites sagittatus T.G. Hartley. 4793 (TYPUS).

Zeugites smilacifolius Scribn. 2023, 2049, 2525, 4713, 5238, 9880.

Zuloagaea bulbosa (Kunth) Bess. 1149, 1362, 1437, 4244, 
4245, 24679.

\section{Family Podostemaceae}

Marathrum foeniculaceum Bonpl. 10269, 11624 (Type of Marathrum elegans Royen), 15952.

\section{Family Polemoniaceae}

Bonplandia geminiflora Cav. 331, 2419, 2494, 2664, 2873, 5108, 5126, 9841, 11276, 11657, 11680, 11832, 12534, 12736, 12826, 13531, 14862, 16041, 16134, 16202.

Cobaea lutea D. Don. 11671.

Cobaea pringlei (House) Standl. 22453, 23217, 23469, 24530, 24881.

Cobaea scandens Cav. 4111, 11945.

Giliastrum incisum (Benth.) J.M. Porter. 17736, 20331, 20566, 21021, 21176, 21885, 23144, 24173, 24422, 28336.

Giliastrum insigne (Brand) J.M. Porter. 27467.

Giliastrum purpusii (Brandegee) J.M. Porter. 21561, 21978.

Giliastrum rigidulum (Benth.) Rydb. 18832, 19221, 19737, 20621, 21082, 25905, 28603, 28740, 28746.

Giliastrum stewartii (I.M. Johnst.) J.M. Porter. 18070, 18187, 18195, 18510, 22798, 28911.

Ipomopsis aggregata (Pursh) V.E. Grant. 17191, 18736, 18919, 20203, 25581.

Ipomopsis macombii (Torr. ex A. Gray) V.E. Grant. 18179, $18233,18745$.

Loeselia mexicana (Lam.) Brand. 18048.

Loeselia amplectens (Hook. \& Arn.) Benth. 10078, 11574, 12880, 13653, 13744, 14941 (Type of Loeselia hintoniorum B.L. Turner), 16160.

Loeselia caerulea (Cav.) G. Don. 17310, 18595, 19015, 19877, 21754, 27895, 28929.

Loeselia ciliata L. 15331.

Loeselia glandulosa (Cav.) G. Don. 123, 206, 2598, 2653, 2725, 2799, 2878, 2999, 3026, 3168, 8813, 11164, 11240, $11303,12743,12890,13483,13621,17522$.

Loeselia grandiflora Standl. 3353.

Loeselia greggii S. Watson. 16839, 17507, 18602, 18649, 19187, 19811, 20079, 20125, 22026, 22083, 24773, 24861, 25563, 25598, 25620, 25881, 27766, 27909, 27995, 28056, 28111, 29055, 29459.

Loeselia mexicana (Lam.) Brand. 262, 3189, 11838, 12707, 15704.

Loeselia pumila (M. Martens \& Galeotti) Walp. 12807, 16191.

Polemonium mexicanum Cerv. ex Lag. 1302, 4397.

Polemonium pauciflorum S. Watson. 17039, 17876, 18872 , 19393, 27543.

\section{Family Polygalaceae}

Hebecarpa barbeyana (Chodat) J.R. Abbot. 18707, 18967, 19733, 20795, 21062, 22767, 22849, 25497, 25867, 27469, 28436, 28511, 28543.

Hebecarpa macradenia (A. Gray) J.R. Abbott. 16587, 17818, 18135, 18729, 19282, 19308, 19340, 19584, 23859, 24250, 24477, 25802, 28306, 28962, 29114.

Hebecarpa palmeri (S. Watson) J.R. Abbott. 28396.
Hebecarpa rivinifolia (Kunth) J.R. Abbott \& J.F.B. Pastore. 4665, 4804, 5014, 5166, 6413, 6670, 6732, 6733, 6742, 10658, 12174, 12217, 13141, 15279.

Monnina ciliolata DC. 15562.

Monnina sylvatica Schltdl. \& Cham. 11064.

Monnina xalapensis Kunth. 251, 2779, 5281, 6739, 7357, 8911, 10169, 10406, 13504, 14160, 17466bis, 26722.

Polygala subalata S. Watson. 18108.

Polygala alba Nutt. 19090, 19253, 22375, 24257, 24433, 28399, 28521, 28634, 28711, 28776.

Polygala aparinoides Hook. \& Arn. 22575, 22920, 23298, 24084, 24531, 24678, 24802, 25067.

Polygala appressipilis S.F. Blake. 1817.

Polygala brachysepala S.F. Blake. 1228, 4518, 4560, 6764, 14630, 15944.

Polygala crinita Chodat. 1810, 9459.

Polygala dolichocarpa S.F. Blake. 23119, 23709, 24003.

Polygala glochidiata Kunth. 1370, 1504, 1844, 4273, 9437, 13105, 13158, 15175.

Polygala gracillima S. Watson. 8379, 9951, 11234, 12656. Polygala hintonii S.F. Blake. 256 (TYPUS), 2331, 2471, 2534, 6851.

Polygala longicaulis Kunth. 1455, 9416.

Polygala minima Pohl ex A.W. Benn. 1369.

Polygala oedophylla S.F. Blake. 18228, 19291, 20958, 21862, 21863, 21976, 22046.

Polygala scoparioides Chodat. 18655, 25960, 27470.

Polygala serpens S.F. Blake. 4283, 6098, 10317.

Polygala shinnersii W.H. Lewis. 17564, 20320, 20724.

Polygala subalata S. Watson. 1557, 4856, 6253, 21766, 21974, 22694, 23642, 23742, 23757, 23863.

Polygala turgida Rose. 17856, 18788, 21949.

Polygala vergrandis W.H. Lewis. 20126, 27336.

Polygala violacea Aubl. 1389, 4468.

Polygala watsonii Chodat. 18941, 19313, 21779, 21874, 22656, 25231.

Rhinotropis lindheimeri (A. Gray) J.R. Abbott. 18227, 18441, 18944, 21148, 24240, 24242, 24264, 25457, 25482, 27628, 28029, 28030.

Rhinotropis minutifolia (Rose) J.R. Abbott. 16539.

Rhinotropis nudata (Brandegee) J.R. Abbott. 25763.

Securidaca diversifolia (L.) S.F. Blake. 3345, 7770, 13749, 15819.

Securidaca sylvestris Schltdl. 3175, 3435, 7789, 14088.

\section{Family Polygonaceae}

Antigonon flavescens S. Watson. 4513, 4726, 5379, 6869, 10041, 10860, 10938, 12287, 12603, 26488.

Antigonon leptopus Hook. \& Arn. 3330, 10870, 14577.

Coccoloba acapulcensis Standl. 14118.

Coccoloba barbadensis Jacq. 10209, 10221, 11781, 13943, 14137, 16033, 16271.

Coccoloba floribunda (Benth.) Lindau. 16132.

Coccoloba humboldtii Meisn. 9101.

Eriogonum atrorubens Engelm. 19002, 19170, 19830, 20432, 27186.

Eriogonum ciliatum Torr. 17316, 19728, 25359, 25896.

Eriogonum greggii Torr. \& A. Gray. 16674, 22156, 23624, 
25956, 29466.

Eriogonum henricksonii Reveal. 25742, 25749.

Eriogonum jamesii Benth. 17197, 18267, 18500, 18930, 20437, 22401, 22801, 27189.

Eriogonum turneri Reveal. 27203, 28595.

Muehlenbeckia tamnifolia (Kunth) Meisn. 1571, 2778, 11139, 14777, 26334, 26363.

Persicaria glabra (Willd.) M. Gómez. 1273, 11609, 21518. Persicaria hydropiperoides (Michx.) Small. 11825, 19951.

Persicaria maculosa Gray. 12197.

Persicaria punctata (Elliott) Small. 5619, 15540, 23822.

Podopterus mexicanus Bonpl. 3775, 5473, 5681, 10051.

Polygonum acuminatum Kunth. 1356.

Polygonum argyrocoleon Steud. ex Kunze. 27876.

Polygonum aviculare L. 2103, 3602, 3688, 4026, 5609, 5767, 19551, 22311.

Rumex acetosella L. 14447, 26541.

Rumex crispus L. 13575, 18549, 20462, 27603, 27611.

Rumex maritimus L. 6034.

Rumex mexicanus Meisn. 664, 4106.

Rumex obtusifolius L. 15510.

Rumex pulcher L. 4910.

Ruprechtia pallida Standl. 15889.

Ruprechtia standleyana Cocucci. 3346, 3386, 5376, 5382, 5386, 10988.

\section{Family Pontederiaceae}

Eichhornia crassipes (C. Mart.) Solms. 9834, 12196.

Heteranthera limosa (Sw.) Willd. 4579, 4680, 6433.

Heteranthera peduncularis Benth. 1983, 4603, 6590, 13202.

Heteranthera reniformis Ruiz \& Pav. 4058.

Heteranthera rotundifolia (Kunth) Griseb. 9402.

\section{Family Portulacaceae}

Portulaca halimoides L. 16672.

Portulaca oleracea L. 4019, 4270, 6461, 9158, 17965, 23017.

Portulaca pilosa L. 6457, 10881, 17661, 19083, 19663, 20539, 20843, 21130, 21160, 21469, 23016, 23165, 23541, 23629, 24432, 24500, 24727, 25145, 25329, 25840, 27379, 28601.

Portulaca suffrutescens Engelm. 9151, 10563.

\section{Family Potamogetonaceae}

Potamogeton pusillus L. 13597.

Stuckenia pectinata (L.) Börner. 5828.

\section{Family Primulaceae}

Anagallis pumila Sw. 5200.

Androsace septentrionalis L. 17292, 17915, 18156, 18202, 18822, 20999.

Ardisia compressa Kunth. 2026, 2430, 2688, 10202, 11322 , 13339 (Type of Ardisia digitata Lundell), 13563, 16265.

Ardisia hintonii Lundell. 13794 (TYPUS).

Ardisia revoluta Kunth. 10092, 10931, 11408, 15320bis, 15921.

Bonellia macrocarpa (Cav.) B. Ståhl \& Källersjö. 5694,
5959, 10332, 15891bis, 16201, 16282.

Bonellia seleriana (Urb. \& Loes. ex Mez) B. Ståhl \& Källersjö. 13389.

Lysimachia arvensis (L.) U. Manns \& Anderb. 304, 841, 3334, 3449, 5651, 11846, 17803, 20344.

Myrsine coriacea (Sw.) R. Br. ex Roem. \& Schult. 352, 1414, 3042, 3088, 7039, 10300, 15667.

Myrsine guianensis (Aubl.) Kuntze. 3034, 3104, 3587.

Myrsine juergensenii (Mez) Ricketson \& Pipoly. 11735, 12395, 14989.

Parathesis macronema Bullock. 10402 (Type of Parathesis hintonii Lundell), 10803 (TYPUS), 14150.

Parathesis melanosticta (Schltdl.) Hemsl. 184, 597, 600, 3144, 3611, 3679, 3921, 4051, 4110, 7683, 8614, 8617, 8753, 11807.

Parathesis mexicana Lundell. 13795 (TYPUS).

Parathesis serrulata (Sw.) Mez. 7190, 13717.

Parathesis tetramera Bullock. 10769, 10779 (TYPUS).

Parathesis villosa Lundell. 13721 (TYPUS).

Samolus cinerascens (B.L. Rob.) Pax \& R. Knuth. 17855, 19086, 21945.

Samolus ebracteatus Kunth. 5647, 18604, 18762, 19939, 20168, 21480, 21577, 21847, 29272.

Samolus floribundus Kunth. 18127.

Synardisia venosa (Mast.) Lundell. 412, 2765, 3719, 7209, 7539, 7676, 11203, 14039, 14167, 15410.

\section{Family Putranjivaceae}

Drypetes lateriflora (Sw.) Krug \& Urb. 25215.

\section{Family Ranunculaceae}

Anemone mexicana Kunth. 3448, 4874, 16071, 26254, 26610, 26627.

Aquilegia elegantula Greene. 17016, 17332, 18125.

Clematis dioica L. 1312, 1680, 1956, 2203, 2496, 2552, 3133, 4256, 4400, 4454, 5105, 6397, 6407, 6408, 9777, $12502,15514,15555,16181,16208,17442$ bis.

Clematis drummondii Torr. \& A. Gray. 16531, 16669, 19552, 20337, 23601, 25469, 27608, 28685.

Clematis grossa Benth. 3028, 5147, 8769.

Clematis pitcheri Torr. \& A. Gray. 21922, 22110, 22290, 22459, 22934, 23055, 23250, 23258, 24826, 25848.

Clematis polygama Jacq. 26720.

Clematis rhodocarpa Rose. 13367.

Delphinium bicornutum Hemsl. 10659, 26036, 26196, 26291.

Delphinium madrense S. Watson. 22774, 24217.

Delphinium subscandens Ewan. 2063, 6579 (TYPUS), 8293.

Delphinium valens Standl. 17043, 17128, 17418, 18883.

Ranunculus dichotomus Moc. \& Sessé ex DC. 543, 4050, 6245, 7976, 8003, 11888, 11938, 17893, 26042, 26185, 26532, 26558.

Ranunculus donianus Pritzel. 4610, 14458.

Ranunculus fasciculatus Sessé \& Moc. 13021.

Ranunculus peruvianus Pers. 17004, 17100, 17350, 18854, 19324, 19390, 20308, 21255, 22221, 22833.

Ranunculus petiolaris Kunth ex DC. 7701, 7715, 13924, 
13985, 17095, 17863, 21954, 26150, 26577, 26641.

Ranunculus praemorsus Kunth ex DC. 11987.

Ranunculus sierrae-orientalis (L. Benson) G.L. Nesom. 14397, 16938, 17372, 19325, 21300, 21612, 24012, 25167, 25235.

Thalictrum grandifolium S. Watson. 16809, 20254, 21303, 22309, 24708, 24827, 25444.

Thalictrum hernandezii Tausch. 4263, 4530, 8126, 15020.

Thalictrum hintonii Boivin. 6743 (TYPUS).

Thalictrum peltatum DC. 1078, 1185, 4259, 4547, 6557, 6740, 8061, 8104, 15071 .

Thalictrum pubigerum Benth. 552, 3851, 4200.

Thalictrum strigillosum Hemsl. 18688.

Family Resedaceae

Oligomeris linifolia (Vahl) J.F. Macbr. 27038, 27071, 28733.

Reseda luteola L. 360, 2436, 11921, 17881, 19371, 27054, 27604.

\section{Family Rhamnaceae}

Ceanothus buxifolius Willd. ex Schult. f. 17030, 17376, 17761, 18269, 18477, 18681, 18844, 21006, 22613, 25094, 25166, 25295.

Ceanothus caeruleus Lag. 545, 546, 637bis, 640, 1098, 6254, 7670, 11291, 12327, 12377, 12785, 12818, 13279, 14500, 15103, 15369, 15516, 16813, 17053, 17401, 17461, Bus (), 17548, 18813, 18900, 18949, 20321, 21008, 22077, 22911, 24322, 25950, 26099, 26273, 26358, 26525, 28071, 28073.

Ceanothus greggii A. Gray. 17574, 18203, 18268, 18478, 18805, 20108, 20154, 21864, 28090.

Ceanothus lanuginosus (M.E. Jones) Rose. 16744.

Colubrina celtidifolia (Schltdl. \& Cham.) Schltdl. 7681, 7684, 7978, 12251, 13929, 14058.

Colubrina ehrenbergii Schltdl. 24502.

Colubrina greggii S. Watson. 17531, 17799, 17819, 21222, 21751, 22173, 24097, 24125, 24247, 24317, 24368, 25242.

Colubrina macrocarpa (Cav.) G. Don. 8854.

Colubrina triflora Brongn. ex G. Don. 1664, 6447, 6610, 6702, 11593, 12089, 15070, 16207.

Condalia ericoides (A. Gray) M.C. Johnst. 16742, 19102, 21969, 27547.

Condalia fasciculata I.M. Johnst. 19586, 20624, 25999, 27561, 28791.

Condalia lycioides (A. Gray) Weberb. 16635, 16805.

Frangula betulifolia (Greene) Grubov. 17801, 18339, 24388.

Frangula hintonii (M.C. Johnst. \& L.A. Johnst.) A. Pool. 1141 (TYPUS).

Frangula mucronata (Schltdl.) Grubov. 17176.

Frangula palmeri (S. Watson) Grubov. 2282.

Gouania lupuloides (L.) Urb. 1283, 3888, 4850, 5139, 7075, 11623, 11784, 12248, 12458.

Gouania polygama (Jacq.) Urb. 6531, 9615, 10926, 14611. Gouania rosei Wiggins. 6617, 6947.

Gouania stipularis DC. 6723, 14087.

Karwinskia humboldtiana (Schult.) Zucc. 7257, 9085, 9993, 16647, 19356, 19896, 20799, 21052, 21151, 21199, 21210,
22808, 22862, 22868.

Karwinskia mollis Schltdl. 4004, 4085, 4352, 10123.

Karwinskia venturae R. Fernández. 3630.

Rhamnus biglandulosa Sessé \& Moc. 6613, 6667, 6944, 6952, 10917.

Rhamnus sharpii M.C. Johnston \& L.A. Johnston. 4082 , 6396.

Sageretia mexicana G.L. Nesom. 3891, 7720.

Sageretia elegans (Kunth) Brongn. 14919.

Sarcomphalus amole (Sessé \& Moc.) Hauenschild. 3795, 5520, 5963, 9038, 10065.

Sarcomphalus lloydii (Standl.) Hauenschild. 16210, 21679, 27559.

Sarcomphalus mexicana (Rose) Hauenschild. 15871.

Sarcomphalus obtusifolius (Hook. ex Torr. \& A. Gray) Hauenschild. 16529, 25786.

Ventia serrata (Schult.) Hauenschild. 651, 866, 1132, 3696, 3862, 23226, 24813, 25285, 25286, 25288.

Ventia standleyana (C.B. Wolf) Hauenschild. 19218.

\section{Family Rosaceae}

Acaena elongata L. 791, 4928, 14461, 26216.

Cercocarpus breviflorus A. Gray. 18693.

Cercocarpus fothergilloides Kunth. 18373, 18388, 18537, 18739, 18946, 19399, 20134, 21827, 21843, 21990, 22032, 23769, 24060, 24164, 24165, 25706, 28062, 28467.

Cercocarpus macrophyllus C.K. Schneid. 17339, 22283, 22543, 22611, 22670, 22690, 24089, 24310, 24814, 25180, 25185, 25290, 26096, 26381.

Cercocarpus mexicanus Henr. 18191 (TYPUS).

Cercocarpus mojadensis C.K. Schneid. 18193, 24378.

Cercocarpus montanus Raf. 16541, 18115, 18702, 19235, 20272, 20961, 27302.

Crataegus aurescens J.B. Phipps. 21376.

Crataegus baroussana Eggl. 18600, 22522, 23436.

Crataegus gracilior J.B. Phipps. 384, 2826, 3491, 3492, 4047, 24608, 24693.

Crataegus greggiana Eggl. 21317, 24680, 29043.

Crataegus mexicana DC. 7067, 15752, 24905.

Crataegus rosei Eggl. 18368, 21254, 21322, 21323, 21442, 21701, 24535, 25265, 25277.

Crataegus tracyi Ashe ex Eggl. 29050.

Fallugia paradoxa (D. Don) Endl. ex Torr. 16591, 27512.

Fragaria mexicana Schltdl. 3499, 15751.

Fragaria virginiana Duchesne. 17015, 18163, 18825, 20981, 22952, 25418, 25608.

Holodiscus argenteus (L. f.) Maxim. 2733, 13280, 15524, 15757, 26023, 26121, 26288, 26448, 26639.

Holodiscus discolor (Pursh) Maxim. 17367, 22228, 22411, 25083.

Lachemilla aphanoides (Mutis ex L. f.) Rothm. 844, 2861, 5351, 12374, 15635, 23141, 23156, 23273.

Lachemilla orbiculata (Ruiz \& Pav.) Rydb. 26305, 26548. Lachemilla procumbens (Rose) Rydb. 21620, 22225.

Lachemilla sibbaldiifolia (Kunth) Rydb. 4887, 15276, 26432.

Lindleya mespiloides Kunth. 16654, 16692, 17052, 17343, 17796, 17815, 18215, 18489, 18696, 18701, 18982, 19451, 
22045, 24144, 27087, 28060, 28141 .

Malacomeles denticulata (Kunth) G.N. Jones. 16653, 17449, 17499, 17665, 18786, 19063, 19898, 20150, 21022, 21329, 21746, 21826, 22724, 23724, 24244, 24505, 28072, 28087, 28142, 28171.

Malacomeles paniculata (Rehder) J.B. Phipps. 18376, 18407, 19350, 22644, 23510, 24027, 25084, 25092, 25122, 29103.

Malacomeles psilantha (C.K. Schneid.) B.L. Turner. 27388, 27487, 27645.

Malus pumila Mill. 18062.

Petrophytum caespitosum (Nutt.) Rydb. 20459, 20500, 20864.

Photinia guerreris J.B. Phipps. 14234 (TYPUS).

Photinia microcarpa Standl. 327, 338 (Type of Photinia microcarpa subsp. hintonii J.B. Phipps), 7233, 8918.

Physocarpus opulifolius (L.) Maxim. 22964.

Potentilla candicans Humb. \& Bonpl. ex Nestl. 788, 4250, 4393.

Potentilla heterosepala Fritsch. 14451.

Potentilla hippiana Lehm. 19071, 27544.

Potentilla indica (Andrews) Th. Wolf. 4197, 24136.

Potentilla leonina Standl. 17035, 18828, 18843, 20210, 25492.

Potentilla macdonaldii B.L. Turner. 26579.

Potentilla ranunculoides Kunth. 1035, 8821, 13016, 17008, 17329, 17354, 18366, 18939, 19369, 26534, 26686.

Potentilla rubra Willd. 1033, 4391, 4903, 6078.

Potentilla staminea Rydb. 15009.

Prunus brachybotrya Zucc. 423, 2442bis, 2865, 3183, 3328, 12676, 13514, 22746, 26430.

Prunus cortapico Kerber ex Koehne. 7145.

Prunus hintonii (C.K. Allen) Kosterm. 13737 (TYPUS).

Prunus mexicana S. Watson. 17282, 18793, 19387, 20215.

Prunus rhamnoides Koehne. 6059, 7374, 10403.

Prunus serotina Ehrh. 243, 425, 1009, 1248, 3684, 16756, 17001, 18714, 21357, 22563, 22668, 22741, 22747, 22755, 24387, 25298.

Prunus tetradenia Koehne. 3590.

Purshia plicata (D. Don) Henr. 16586, 16737, 17573, 17656, 19660, 19689, 21988, 22711, 23342, 23532, 23581, 23626, 24050.

Pyrus communis L. 3476.

Rosa banksiae W.T. Aiton. 3556.

Rosa carolina L. 17576, 19151, 21711.

Rosa multiflora Thunb. ex A. Murray. 5932, 7465.

Rubus aboriginum Rydb. 24087, 24091.

Rubus adenotrichos Schltdl. 3485, 10433, 11834, 14081, 14973, 15675.

Rubus caudatisepalus Calderón. 22825.

Rubus coriifolius Liebm. 3443, 9890, 10699, 14970.

Rubus flagellaris Willd. 18411, 19152, 22820, 23324, 24078, 24135

Rubus pringlei Rydb. 4907.

Rubus pumilus Focke. 611, 8906.

Rubus strigosus Michx. 481, 3251, 17300.

Rubus trilobus Moc. \& Sessé ex Ser. 14433, 26181, 26569.

Sericotheca dumosa (Nutt.) Rydb. 11122, 17193, 17916, 18289, 18889.
Vauquelinia corymbosa Bonpl. 16521, 16693, 19087, 19097, 19357, 21989, 22704, 23494, 24455, 25918, 27793, 27800, 28121.

\section{Family Rubiaceae}

Arachnothryx jurgensenii (Hemsl.) Borh. 1280, 1420, 2705, 4408, 4517, 6394, 7016, 7042, 7895, 8082, 10493.

Arachnothryx leucophylla (Kunth) Planch. 10936, 12737, 12840, 13638, 14738.

Arcytophyllum fasciculatum (A. Gray) Terrell \& H. Rob. 16506, 16859, 22002, 23373, 23584, 25764, 27455, 29356. Augusta rivalis (Benth.) J.H. Kirkbr. 13790.

Balmea stormiae Martínez. 7894.

Borreria suaveolens G. Mey. 673, 1645.

Borreria verticillata (L.) G. Mey. 15210, 15456, 29702.

Bouvardia hintoniorum B.L. Turner. 15408.

Bouvardia capitata Bullock. 1268, 2657, 6319 (TYPUS), 8092, 8140, 8193, 8552, 8564, 8600.

Bouvardia cordifolia DC. 4078, 7893, 7950, 8433, 15003, 17216.

Bouvardia chrysantha Mart. 1131 (Type of Bouvardia cataphyllaris Bullock), 8544, 8761, 9565, 11102, 13917.

Bouvardia dictyoneura Standl. 10759.

Bouvardia laevis M. Martens \& Galeotti. 932bis, 7508, 8537, 15048.

Bouvardia loeseneriana Standl. 8107 (Type of Bouvardia hintonii Bullock).

Bouvardia multiflora (Cav.) Schult. \& Schult. f. 989, 1113, 7573, 7898, 7944.

Bouvardia obovata Kunth. 1079, 4404.

Bouvardia standleyana W.H. Blackw. 12677 (TYPUS).

Bouvardia ternifolia (Cav.) Schltdl. 294, 801, 908, 4094, 8470, 11808bis, 11812, 16534, 16639, 18513, 19598, 20387 , 20836, 22548, 25748, 25839, 29445.

Cephalanthus occidentalis L. 14123.

Cephalanthus salicifolius Bonpl. 920, 4139, 6752, 10208, 15166.

Coccocypselum hirsutum Bartl. ex DC. 10774, 11042.

Coffea arabica L. 948.

Crusea diversifolia (Kunth) W.R. Anderson. 29715.

Crusea longiflora (Willd. ex Roem. \& Schult.) W.R. Anderson. 9547.

Crusea calocephala DC. 1739, 1879, 1961, 26322.

Crusea coccinea DC. 544, 641, 4461, 7954, 9740, 10155, 10721, 10727, 11066, 11077, 11190, 11923, 12396, 14651, 17213, 26276.

Crusea coronata B.L. Rob. \& Greenm. 8206, 8222, 8440, 8447, 8530, 9452.

Crusea diversifolia (Kunth) W.R. Anderson. 1845, 4859, 7995, 25580, 27242, 27700, 28905.

Crusea hispida (Mill.) B.L. Rob. 716, 1799, 2024, 4848 (Type of Crusea grandiflora Paul G. Wilson), 7810, 8365, 8466, 8489, 8532, 8565, 8726, 14848, 15112.

Crusea longiflora (Willd. ex Roem. \& Schult.) W.R. Anderson. 1561, 8294, 15259, 15455, 15604.

Crusea parviflora Hook. \& Arn. 2669, 5366, 11158.

Crusea psyllioides (Kunth) W.R. Anderson. 1467, 1963, 8531,15200 . 
Chiococca alba (L.) Hitchc. 749, 949, 1014, 1016, 3427, 3901, 10494, 13898, 14365, 15855, 17728, 25006.

Chiococca pachyphylla Wernham. 16902, 21559, 24441.

Chiococca phaenostemon Schltdl. 2048, 3681.

Chomelia protracta (Bartl. ex DC.) Standl. 10634, 10637, 14356.

Deppea grandiflora Schltdl. 3699, 15108, 26615.

Deppea hintonii Bullock. 4824 (TYPUS), 8128.

Deppea pubescens Hemsl. 7402.

Didymaea alsinoides (Schltdl. \& Cham.) Standl. 17151, 17167.

Didymaea mexicana Hook. f. 540, 1304, 1725, 3690, 4071, 4075, 4205, 15099.

Diodia teres Walter. 8449, 15216.

Exostema caribaeum (Jacq.) Roem. \& Schult. 5561, 6297, 6341, 6931, 7056, 7124.

Galium juniperinum Standl. 28586, 29890.

Galium mexicanum Kunth. 29704.

Galium triflorum Michx. 18051.

Galium aparine L. 798, 4100, 18710.

Galium dempsterae B.L. Turner. 23110, 25526.

Galium hintoniorum B.L. Turner. 24804, 25127 (TYPUS).

Galium juniperinum Standl. 18731, 18748, 21587, 24468.

Galium mexicanum Kunth. 1509, 1604, 13147, 15234, 18290 ,

26243, 26383, 26598, 26753.

Galium oresbium Greenm. 27092.

Galium rzedowskii Dempster. 20582, 21330, 22274.

Galium seatonii Greenm. 4924.

Galium uncinulatum DC. 1727, 4087, 16817, 17754, 18880, 19033, 19147, 19710, 20242, 20262, 21029, 21916, 22208, 22414, 22895, 22976, 23157, 23176, 23439, 24128, 24410, 29038.

Gonzalagunia panamensis (Cav.) K. Schum. 14146, 16128. Guettarda elliptica Sw. 5308, 7816, 7902, 8012, 10094, 10120, 10409, 16122.

Hamelia patens Jacq. 25139, 25307.

Hamelia versicolor A. Gray. 1059, 3917, 7596, 7658, 7771, 12629.

Hamelia xorullensis Kunth. 1085, 1612, 3560, 7157, 10198, 11589, 12530, 13863.

Hedyotis acerosa A. Gray. 25768.

Hedyotis nigricans (Lam.) Fosberg. 16540, 18278 (Type of Hedyotis nigricans var. gypsophila B.L. Turner), 18483, 18517, 18715, 18744, 18979, 19690, 19810, 20007, 20378, 22017, 22092, 24351, 24373, 24775, 25434, 25940, 25948, 27081, 27183, 27885, 27949, 28172, 28407, 28579, 28621. Hedyotis palmeri (A. Gray) W.H. Lewis. 18218, 18743, 18993, 20412, 22861, 22871, 24476, 25449, 28027, 28330, 28960 .

Hedyotis rubra (Cav.) A. Gray. 16547, 18221, 18451, 19528, 21933, 28996.

Hedyotis teretifolia (Terrell) G.L. Nesom. 29350, 29411, 29418.

Hedyotis wrightii (A. Gray) Fosberg. 1044, 8005.

Hintonia latiflora (Sessé \& Moc. ex DC.) Bullock. 1258 (Type of Hintonia latiflora var. leiantha Bullock), 3413, 4317, 6333, 6922, 6960, 7006, 7097, 7125, 7859bis, 7876, $8095,8123,8139,10217,10338,12004,12541,15948$,
15960, 15961, 15962.

Hoffmannia cuneatissima B.L. Rob. 7777, 17077.

Mitracarpus breviflorus A. Gray. 5653.

Mitracarpus hirtus (L.) DC. 11436, 13192, 14654, 25878.

Mitracarpus schizangius DC. 5259.

Paederia ciliata (Bartl. ex DC.) Standl. 1195, 4123, 4365, 4738, 7163, 7234, 8047, 8049, 8055, 8078, 8118, 8159, 13085.

Palicourea chiapensis (Standl.) Borh. 10778, 11191.

Posoqueria latifolia (Rudge.) Roem. \& Schult. 14360.

Psychotria horizontalis Sw. 13912.

Psychotria trichotoma M. Martens \& Galeotti. 14598.

Randia aculeata L. 13873, 17726, 17931.

Randia armata (Sw.) DC. 10308.

Randia capitata DC. 7501, 7506, 7510, 10138.

Randia cinerea (Fernald) Standl. 10239, 10240, 10241, 10267, 13899, 14348.

Randia cookii Standl. 12722.

Randia echinocarpa Sessé \& Moc. ex DC. 4271, 5965, 5970, 7121, 7154, 7241, 7745, 7817, 7840, 7844, 7852, 7855, 7867, 7875, 7882, 7890, 7903, 13783.

Randia laetevirens Standl. 24147, 24634, 24776, 25260.

Randia obcordata S. Watson. 17940.

Randia pringlei (S. Watson) A. Gray. 16601, 24768, 25735, 25736, 25747, 27460.

Randia tetracantha (Cav.) DC. 10463.

Randia thurberi S. Watson. 5853.

Relbunium laevigatum (DC.) Hemsl. 19003, 19469, 20237, 20438.

Richardia scabra L. 595, 703, 1010, 5470, 7359, 7557, 7585, 7805, 12182, 12243, 12317, 12460, 12870, 13191, 13870, 15209, 15958.

Rogiera amoena Planch. 14853, 14931.

Rovaeanthus strigosus (Benth.) Borh. 12886.

Simira mexicana (Bullock) Steyerm. 4773, 4818 (TYPUS), 6108, 6917, 7043, 7173, 9836, 10391, 11245, 15987.

Solenandra mexicana (A. Gray) Borh. 16248.

Sommera grandis (Bartl. ex DC.) Standl. 13940.

Spermacoce tenuior L. 766, 2472, 2593, 12527, 13135, 13168, 17476bis, 20324, 20351, 23297, 23430, 23519.

Spermacoce tetraquetra A. Rich. 26504.

\section{Family Rutaceae}

Amyris marshii Standl. 27799.

Amyris mexicana Lundell. 13763 (TYPUS).

Amyris rekoi S.F. Blake. 10249, 10509, 14077.

Amyris staminosa Lundell. 13800 (TYPUS).

Casimiroa edulis La Llave \& Lex. 3077, 3120.

Casimiroa pringlei (S. Watson) Engler. 18121, 23759, 24057, 24059, 24064.

Casimiroa sapota Oerst. 2820, 13385.

Casimiroa tetrameria Millsp. 326, 3208.

Citrus medica L. 5356, 6186.

Choisya palmeri A. Gray. 18465, 18705, 21897, 27013, 27180, 28220, 28221, 28241, 28264, 28267, 28268, 29005. Decatropis bicolor (Zucc.) Radlk. 17673, 20804, 21540, 22368, 24346.

Esenbeckia berlandieri Baill. ex Hemsl. 10924, 24777. 
Helietta parvifolia (A. Gray ex Hemsl.) Benth. 16831, 17587, 17941, 23878, 24960, 24966.

Murraya paniculata (L.) Jack. 4290.

Pilocarpus racemosus Vahl. 16178.

Ptelea trifoliata L. 16728, 17745, 17792, 18259, 18416, 19133, 19384, 21066, 21084, 21918, 22720, 27988, 28107, 28196, 28216.

Ruta chalepensis L. 20906.

Thamnosma texana (A. Gray) Torr. 25888, 27626.

Thamnosma stanfordii I.M. Johnst. 16729.

Thamnosma texana (A. Gray) Torr. 20124, 21785.

Zanthoxylum fagara (L.) Sarg. 3342, 4559, 10555, 17742 , 21467, 22732, 22805, 24070, 25673, 27741.

Zanthoxylum limoncello Planch. \& Oerst. 4126.

Zanthoxylum mollissimum (Engl.) P. Wilson. 4064, 9337, 10136, 10421, 14695.

\section{Family Sabiaceae}

Meliosma dentata (Liebm.) Urb. 2797, 3513, 8431, 11924, 13277, 17152.

\section{Family Salicaceae}

Casearia aculeata Jacq. 3344, 6320.

Casearia bartlettii Lundell. 15964 (Type of Casearia hintonii Lundell).

Casearia javitensis Kunth. 10313.

Casearia nitida (L.) Jacq. 776, 3196, 3406, 3785, 3813, 4131, 5686, 10271, 10998, 11566, 13760, 13761, 13935, 14064, 15803, 15858, 15963, 16306.

Casearia sylvestris Sw. 10625.

Neopringlea integrifolia (Hems1.) S. Watson. 19996, 20294, 21549, 21770, 22734, 23996, 24976.

Populus alba L. 3477, 3547, 3868.

Populus tremuloides Michx. 17976.

Prockia crucis P. Browne ex L. 760, 4277, 4509, 6538, 10253, 13843, 14331.

Salix bonplandiana Kunth. 1135, 2603, 8683, 9215, 9924, 9926, 11599, 14830, 15150bis, 26731.

Salix humboldtiana Willd. 3289, 5682.

Salix lasiolepis Benth. 1719.

Salix nigra Marshall. 21924, 24095.

Salix paradoxa Kunth. 3261, 3591, 5921, 8961, 8992, 10167, 14217, 14268, 15417, 16733, 17020, 18201, 18207, 18826, 22754.

Salix taxifolia Kunth. 2787, 2967, 3182, 5586, 8795, 12853, 15303, 15864, 16141.

Xylosma flexuosa (Kunth) Hemsl. 12806, 14965, 15512, 16209, 17589, 17743, 21550, 27706.

Xylosma panamensis Turcz. 2394, 2819, 2838, 3062, 3239, 5344, 13589.

Xylosma velutina (Tul.) Triana \& Planch. 742, 3820, 3920, 7429, 10426.

\section{Family Santalaceae}

Arceuthobium blumeri A. Nelson. 17273.

Arceuthobium douglasii Engelm. 17271.

Arceuthobium vaginatum (Humb. \& Bonpl. ex Willd.) J. Presl. 3272, 8901, 17023.
Phoradendron bolleanum (Seem.) Eichler. 17904, 19716. Phoradendron brachystachyum (DC.) Nutt. 417, 3148, 6063, 10407.

Phoradendron carneum Urb. 3071, 5370.

Phoradendron lanceolatum Engelm. ex A. Gray. 17283, 17363, 17758.

Phoradendron leucarpum (Raf.) Reveal \& M.C. Johnst. 27058.

Phoradendron piperoides (Kunth) Trel. 10597.

Phoradendron quadrangulare (Kunth) Griseb. 9099.

Phoradendron robinsonii Urb. 3387, 5888.

\section{Family Sapindaceae}

Acer negundo L. 16748, 21851.

Allophylus cominia (L.) Sw. 24786.

Cardiospermum dissectum (S. Watson) Radlk. 24251.

Cardiospermum halicacabum L. 1940, 3157, 3744, 5140, 5644, 6887, 7014, 7095, 7099, 7253, 7555, 9613, 9807, $11521,13291,15146,16906,17614,20532$, 20588, 21158, 21209, 21410, 21448, 24202, 26476, 26484.

Cupania glabra Sw. 10122, 10786, 14000, 14063, 16152.

Dodonaea viscosa (L.) Jacq. 295, 1111, 4869, 8974, 9697, 10307, 11712, 21824, 22761.

Houssayanthus biternatus (Weath.) Rzed. \& Calderón. 3039, 4661.

Paullinia cururu L. 10339, 11343.

Paullinia pinnata L. 14592.

Paullinia sessiliflora Radlk. 3366, 5696, 7522, 7593, 13581.

Paullinia tomentosa Jacq. 24204.

Sapindus saponaria L. 3349, 5023, 5529, 5722, 5785, 6047, 7293, 14842, 17784, 17957, 24203.

Serjania brachycarpa A. Grayex Raldk. 25004.

Serjania corindifolia Radlk. 16904.

Serjania mexicana (L.) Willd. 455, 2671, 3031, 3198, 3352, 5903, 7212, 7304, 7330, 7365, 7622, 13544.

Serjania paniculata Kunth. 11741, 11759.

Serjania racemosa Schumach. 9522, 25007.

Serjania schiedeana Schltdl. 12457, 12561.

Serjania trifoliolata Radlk. 2874, 3005.

Serjania triquetra Radlk. 1264, 2218, 6883, 7052, 7061, 13189, 14476, 15148.

Thouinia acuminata S. Watson. 6187, 12323.

Thouinia villosa DC. 2424, 2606, 5573, 7033, 7073, 7105, 7151, 7215, 11279, 13511, 14849.

Thouinidium decandrum (Bonpl.) Radlk. 5690, 5867, 7588, 9994, 9998, 10010, 10398, 10436, 14003.

Ungnadia speciosa Endl. 17519bis, 17519, 17739, 28164. Urvillea ulmacea Kunth. 2488, 2592, 5120, 7004, 7047, 17604, 21420, 24156, 24205.

\section{Family Sapotaceae}

Manilkara zapota (L.) P. Royen. 4597.

Pouteria campechiana (Kunth) Baehni. 3210, 4145, 5391, 5940, 6116, 9185.

Sideroxylon capiri (A. DC.) Pittier. 746, 3833, 5879, 5882, 5974, 13911.

Sideroxylon lanuginosum Michx. 25911, 28984. 
Sideroxylon portoricense Urb. 7700, 10416.

\section{Family Saururaceae}

Anemopsis californica (Nutt.) Hook. \& Arn. 16797, 17861, 21948.

\section{Family Saxifragaceae}

Heuchera amoena Rosend., Butters \& Lakela. 17340.

Heuchera hemsleyana Rosend., Butters \& Lakela. 8933.

Heuchera longipetala Moc. ex Ser. 522, 877, 3518, 8942, 15571.

Heuchera mexicana W. Schaffn. ex Small \& Rydb. 22438, 25196, 25281.

Heuchera orizabensis Hemsl. 17530bis.

Heuchera rubescens Torr. 17146, 18952, 19123, 22974, 28572.

Heuchera sanguinea Engelm. 17912, 18331, 18855, 20302.

\section{Family Scrophulariaceae}

Alonsoa meridionalis (L. f.) Kuntze. 9947, 14748, 26057, 26233, 26272, 26513.

Buddleja cordata Kunth. 29807, 29824.

Buddleja parviflora Kunth. 18049, 18104, 29794.

Buddleja sessiliflora Kunth. 29833.

Buddleja americana L. 15615.

Buddleja cordata Kunth. 394, 2813, 7600, 13458, 14753, $16662,16819,17186,17600,17699,19214,19800,21086$, 21207, 21397, 21752, 22412, 23756, 23802, 24392, 24985, 26031, 26108, 26110.

Buddleja marrubiifolia Benth. 16537, 16710, 18077, 19715, 23348, 25754, 27376, 27449, 28413, 28482.

Buddleja parviflora Kunth. 108, 371, 616, 2129, 2678, 8459, 9744, 12787, 13255, 14314, 14791, 15580, 26070.

Buddleja scordioides Kunth. 16515, 16668, 18559, 19769 , 29260.

Buddleja sessiliflora Kunth. 381, 398, 2623bis, 2623, 2831, 2960, 5700, 10131, 12964, 14111, 17533bis, 18141, 25190.

Capraria biflora L. 1979, 3825, 5474, 26492.

Capraria frutescens (Mill.) Britton. 3383, 4346, 12824, 12876, 16112.

Leucophyllum revolutum Rzed. 27382.

Leucophyllum alejandrae G.L. Nesom. 22321 (TYPUS), 25988, 28608, 28749, 29388, 29879, 29880.

Leucophyllum candidum I.M. Johnst. 16569, 23566, 27078 , 27079, 27504, 28453, 28481, 28837, 28865, 29324, 29452, 29468, 29481, 29482, 29598.

Leucophyllum coahuilensis Henr. 28370, 28833, 28877, 29600.

Leucophyllum frutescens (Berland.) I.M. Johnst. 16829, 21476, 23611, 23616, 23986, 24180, 24719, 25225, 25522, 25787, 25873, 27505, 27527, 27735, 28309, 28416, 28764. Leucophyllum hintoniorum G.L. Nesom. 20929 (TYPUS), 21961, 21973, 22567, 23024, 23128, 23131, 23841, 25156, 25161, 25723, 27068, 29139, 29141.

Leucophyllum laevigatum Standl. 16505, 16820, 18461, 18504, 18544, 19750, 21060, 22809, 24755, 27090, 27095, 27148, 27149, 27440, 27441, 27442, 27447, 27457, 27597, 27598, 29118, 29130, 29239, 29251.
Leucophyllum langmaniae Flyr. 24952, 25903, 25952.

Leucophyllum minus A. Gray. 25738, 25757, 27500, 29289 , 29335.

Leucophyllum pruinosum I.M. Johnst. 22565, 23770, 23890, 27105, 27367.

Leucophyllum revolutum Rzed. 27332, 27382, 27384, 27696, 28556.

Leucophyllum zygophyllum I.M. Johnst. 17484, 17640, 21599, 22335, 23023, 23600, 23865, 25162, 27102, 27106, 27107, 27109, 28218, 28219, 28555.

\section{Family Schoepfiaceae}

Schoepfia pringlei B.L. Rob. 1694, 2956, 3252, 5072, 5420.

Schoepfia schreberi J.F. Gmel. 3385, 17749.

Family Simaroubaceae

Castela erecta Turpin. 22710.

Quassia amara L. 15865, 15866, 16221.

\section{Family Siparunaceae}

Siparuna thecaphora (Poepp. \& Endl.) A. DC. 10771, 10814, $11705,14649$.

\section{Family Smilacaceae}

Smilax aristolochiifolia Mill. 14038, 25274, 25938.

Smilax bona-nox L. 18685, 19115, 19149.

Smilax laurifolia L. 10360, 11627, 17711.

Smilax moranensis M. Martens \& Galeotti. 408, 3478, 3523, 4041, 17648.

Smilax pringlei Greenm. 293, 3543, 7620, 17099.

Smilax subpubescens A. DC. 14364 (Type of Smilax occidentalis C.V. Morton).

Smilax tamnoides L. 24079.

\section{Family Solanaceae}

Bouchetia erecta DC. ex Dunal. 8827, 18260, 18467, 18882, 22018, 23090, 23148, 24400, 25393, 25568.

Brachistus stramoniifolius (Kunth) Miers. 14065.

Browallia americana L. 300, 5353, 12731.

Brugmansia sanguinea (Ruiz \& Pav.) D. Don. 4204.

Calibrachoa parviflora (A. Juss.) D'Arcy. 5547, 5848, 16727, 16807, 18603.

Capsicum annuum L. 5085, 8119, 21542, 24206.

Capsicum rhomboideum (Dunal) Kuntze. 9311, 11977, 17588, 17943, 21500, 23896.

Cestrum thyrsoideum Kunth. 29812.

Cestrum alternifolium (Jacq.) O.E. Schulz. 3171, 3651.

Cestrum flavescens Greenm. 14255, 19331, 21295, 21817, 22623, 22677.

Cestrum flavinervium Francey. 11681.

Cestrum laxum Benth. 7476, 11086, 13457, 17505.

Cestrum nitidum M. Martens \& Galeotti. 534, 2794, 2919, 10949, 17436bis.

Cestrum nocturnum L. 603, 1051, 4027, 8633, 26004.

Cestrum roseum Kunth. 246, 1102, 3612, 9000, 9012, 11804.

Cestrum thyrsoideum Kunth. 152, 273, 495, 2097, 2923, 
3700, 13411, 17473bis.

Cestrum tomentosum L. f. 3095, 3450, 3873, 8855, 8891, 11299, 11701, 12935, 14052.

Chamaesaracha arida Henr. 28822.

Chamaesaracha coniodes (Moric. ex Dunal) Britton. 19654, 25785.

Chamaesaracha coronopus (Dunal) A. Gray. 19499, 20477, 20625, 24756, 25483, 25506, 25889, 27565, 27629.

Chamaesaracha crenata Rydb. 16623, 23372, 23376.

Chamaesaracha geohintonii Averett \& B.L. Turner. 28619

(TYPUS), 29247.

Chamaesaracha villosa Rydb. 27425, 28462, 28840.

Datura innoxia Mill. 5650, 17610, 19272, 21447.

Datura kymatocarpa A.S. Barclay. 8173 (TYPUS).

Datura quercifolia Kunth. 16880, 19537, 29013.

Datura stramonium L. 536, 1671, 3877, 5181, 8030, 9599, 15536, 21677.

Hunzikeria texana (Torr.) D'Arcy. 16622, 16897, 27834.

Jaltomata procumbens (Cav.) J.L. Gentry. 7986, 8044, 8050, $8056,8073,8085,8089,8144,14440,18522,22310,23161$, 24702.

Juanulloa mexicana (Schltdl.) Miers. 10244, 10247, 15925. Lycianthes acapulcensis (Baill.) D'Arcy. 10315.

Lycianthes arrazolensis (J.M. Coult. \& Donn. Sm.) Bitter. 1089, 4371, 6159, 6357, 26622.

Lycianthes ciliolata (M. Martens \& Galeotti) Bitter. 971, 1211, 4593, 13994.

Lycianthes dejecta (Fernald) Bitter. 3029, 10517, 21019.

Lycianthes hintonii E. Dean. 22882 (TYPUS), 23263.

Lycianthes moziniana (Dunal) Bitter. 972, 4550, 22222, 22291, 22937 (Type of Lycianthes moziniana var. margaretiana E.A. Dean), 23168, 23237.

Lycianthes pilifera (Benth.) Bitter. 1548, 8102, 15037, 16005.

Lycianthes pringlei (B.L. Rob. \& Greenm.) Bitter. 8750.

Lycianthes stephanocalyx (Brandegee) Bitter. 10849.

Lycium berlandieri Dunal. 16625, 16884, 17850, 18046, 18099, 19560, 19603, 20643, 23282, 25831, 27552, 28463, 28662, 28756.

Lycium californicum Nutt. ex A. Gray. 25832, 25882, 27357.

Lycium leiospermum I.M. Johnst. 25992, 28755.

Lycium pallidum Miers. 25985, 27550.

Lycium parishii A. Gray. 28626, 28778.

Lycium schaffneri Hemsl. 25484.

Nectouxia formosa Kunth. 789, 17928.

Nicandra physalodes (L.) Gaertn. 1834, 5271, 6857, 9888, 13431, 13883.

Nicotiana glauca Graham. 5798, 9324, 10060, 11982, 12499 , 12973, 16670.

Nicotiana nudicaulis S. Watson. 17724, 19346, 19376, 20167, 21842.

Nicotiana obtusifolia M. Martens \& Galeotti. 6000.

Nicotiana palmeri A. Gray. 16661, 17395, 18512, 19623, 19856, 21723.

Nicotiana plumbaginifolia Viv. 1172, 5628, 5755, 7576, 12223, 13591.

Nicotiana repanda Willd. ex Lehm. 21672, 23774, 25668.
Nicotiana tabacum L. 329, 588, 1669, 4113, 5670, 10139.

Physalis orizabae Dunal. 18027.

Physalis cinerascens (Dunal) Hitchc. 18256, 20341, 20774, 21171, 21221, 21449, 23335, 23708, 24350, 25381, 28977 , 29199.

Physalis coztomatl Moc. \& Sessé ex Dunal. 14299, 26596.

Physalis glutinosa Schltdl. 4202.

Physalis gracilis Miers. 23167.

Physalis hederifolia A. Gray. 18978, 22995, 25981, 27429, 28460, 28501, 28781, 28861, 28886.

Physalis hintonii Waterf. 8457 (TYPUS), 17403, 21314, 22212.

Physalis hirsuta M. Martens \& Galeotti. 8493.

Physalis hunzikeriana M. Martínez. 22104 (TYPUS), 22255, 22832, 22900, 23229, 23437, 23500.

Physalis lagascae Roem. \& Schult. 2717, 5204, 5821, 8034, 8418, 8606, 14536.

Physalis microphysa A. Gray. 16703, 21977, 22345, 22355, 23105.

Physalis nicandroides Schltdl. 8174, 8195, 8503, 27781.

Physalis orizabae Dunal. 476, 570, 8472, 13156, 17347.

Physalis patula Mill. 1740, 4592.

Physalis peruviana L. 613, 3431, 4402, 11449, 17182.

Physalis philadelphica Lam. 1613, 4034, 15035, 17594, 27055.

Physalis pringlei Greenm. 2088, 7717, 8057, 8964.

Physalis pubescens L. 5982, 11541, 25842.

Physalis solanacea (Schltdl.) Axelius. 21440, 23498.

Physalis sordida Fernald. 17930, 18464, 18558, 21067 , 26507.

Physalis virginiana Mill. 19502.

Physalis viscosa L. 16620.

Physalis walteri Nutt. 20183.

Solandra nitida Zuccagni. 3016, 11956, 17802, 22931, 22945, 23247, 23836, 25299, 26614.

Solanum cardiophyllum Lindl. 29733.

Solanum nigrescens M. Martens \& Galeotti. 18028.

Solanum stoloniferum Schltdl. \& C.D. Bouché. 18002.

Solanum adscendens Sendtn. 4219, 4470, 6481, 9143, 10557, 12057.

Solanum aligerum Schltdl. 365, 3852, 26251, 26606, 26825, 26834.

Solanum americanum Mill. 399, 1100, 2917, 3855, 5709, 7566, 11898, 12976, 15638, 16619.

Solanum angustifolium Mill. 2167, 2545, 5183.

Solanum aphyodendron S. Knapp. 26373, 26510, 26624.

Solanum appendiculatum Humb. \& Bonpl. ex Dunal. 363, 1101, 2912, 11872, 17161.

Solanum bicolor Willd. ex Roem. \& Schult. 1020.

Solanum bicorne Dunal. 13847, 13906.

Solanum bulbocastanum Dunal. 4246.

Solanum candidum Lindl. 1594, 8170, 9823.

Solanum capsicoides All. 11967.

Solanum cardiophyllum Lindl. 3619, 13992.

Solanum citrullifolium A. Br. 24733.

Solanum chrysotrichum Schltdl. 26168, 26522, 26719.

Solanum demissum Lindl. 11969.

Solanum diphyllum L. 6909, 13903, 15869, 15950. 
Solanum donianum Walp. 3358, 5697, 15872.

Solanum dulcamaroides Dunal. 633, 835, 1360, 1697, 10516, 11857, 13951.

Solanum elaeagnifolium Cav. 16627, 19405, 19440.

Solanum erianthum D. Don. 16905, 17602, 17752, 21215, 22527, 23651, 23830, 24226, 27817.

Solanum fendleri A. Gray. 23504.

Solanum grayi Rose. 444, 1070, 15164.

Solanum hazenii Britton. 10116, 15538.

Solanum hintonii Correll. 4416 (TYPUS), 14403.

Solanum lanceifolium Jacq. 13832.

Solanum laurifolium Mill. 302, 639.

Solanum laxum Spreng. 10494bis, 12255.

Solanum lycopersicum L. 5813.

Solanum malacothrix S. Knapp. 10248, 10418 (TYPUS).

Solanum marginatum L. f. 7948, 8676, 11952.

Solanum minensis C.V. Morton. 14179 (TYPUS).

Solanum morelliforme Bitter \& Münch. 6813.

Solanum myriacanthum Dunal. 21421, 21551.

Solanum nigrescens M. Martens \& Galeotti. 9288, 17607, 18570, 20557, 20645, 20884, 20941, 21125, 21270, 21381, 21479, 21669, 22227, 22286, 22301, 22352, 22659, 23040, 23277, 23480, 27998.

Solanum nigricans M. Martens \& Galeotti. 254, 389, 2827, 3145, 3701, 7479, 9004, 11911, 12747, 15537.

Solanum oxycarpum Schiede. 13995 (Type of Solanum oxycarpum var. brachycarpum Correll).

Solanum pectinatum Dunal. 14579.

Solanum plumense Fernald. 10588.

Solanum pseudocapsicum L. 23285, 26278, 26662, 26797, 26806.

Solanum pubigerum Dunal. 272, 855.

Solanum refractum Hook. \& Arn. 4312, 4320, 6783, 10967, 15976.

Solanum rostratum Dunal. 9711, 11928, 11959, 14600, 16851, 18074, 19580, 19659, 20001, 28955.

Solanum rudepannum Dunal. 420, 1001, 2824, 11182, 13657, 14547.

Solanum stoloniferum Schltdl. \& C.D. Bouché. 14438.

Solanum tenuipes Bartlett. 22874, 23021, 27478, 28483, 28856.

Solanum torvum Sw. 11900.

Solanum triquetrum Cav. 16775, 16855, 20406, 21115.

Solanum tuberosum L. 612, 1047.

Solanum umbellatum Mill. 13666, 15980.

Solanum verrucosum Schltdl. 11929, 15096, 17198, 18894, 19703, 20434, 20786, 21114, 23314, 23326, 25851, 28705.

Witheringia mexicana (B.L. Rob.) Hunz. 17690, 17944, 20592, 21239, 21690, 22893, 24227, 24450, 24614, 24706, 24916, 25150.

\section{Family Stegnospermataceae}

Stegnosperma scandens (Lunan) Standl. 3764, 5431, 5719, $5962,12627$.

\section{Family Styracaceae}

Styrax argenteus C. Presl. 5309, 6145, 6193, 7410, 7618, $11831,14409,14750$.
Styrax ramirezii Greenm. 248, 3712, 3798 (Type of Styrax hintonii Bullock), 5282, 7605, 7685, 10400, 14742.

\section{Family Surianaceae}

Recchia mexicana Moc. \& Sessé ex DC. 5714.

Family Symplocaceae

Symplocos citrea Lex. ex La Llave \& Lex. 144, 264, 2342, 3711, 5092, 5093, 7540, 7615, 13349, 15028, 15367, 15511, 17438bis.

Symplocos hintonii Lundell. 14276 (TYPUS).

\section{Family Talinaceae}

Talinum fruticosum (L.) Juss. 14585.

Talinum paniculatum (Jacq.) Gaertn. 1077, 4088, 10486, 10579, 13047, 13048, 14534, 15203, 25833.

Family Tamaricaceae

Tamarix chinensis Lour. 28800.

\section{Family Thymelaeaceae}

Daphnopsis americana (Mill.) J.R. Johnst. 3173, 3313, 3348, 4062.

Dirca mexicana G.L. Nesom \& Mayfield. 25197, 25276, 25282 .

Family Typhaceae

Typha domingensis Pers. 3908, 17902.

Typha latifolia L. 837, 5878.

\section{Family Ulmaceae}

Ulmus pumila L. 24720.

\section{Family Urticaceae}

Cecropia obtusifolia Bertol. 3568.

Discocnide mexicana (Liebm.) Chew. 634, 638, 3417, 3676 .

Laportea canadensis (L.) Wedd. 23202, 23252, 23982.

Myriocarpa cordifolia Liebm. 3780, 4329, 7519.

Myriocarpa longipes Liebm. 15809.

Parietaria debilis G. Forst. 8473.

Parietaria decoris N.G. Mill. 18123.

Parietaria macrophylla B.L. Rob. \& Greenm. 1334, 8953.

Parietaria pensylvanica Muhl. ex Willd. 2901, 3856, 4699, 28990.

Phenax hirtus (Sw.) Wedd. 2092, 2297, 5219, 5239, 17440bis.

Phenax mexicanus Wedd. 14053.

Pilea acuminata Liebm. 2371.

Pilea dauciodora Wedd. 4296.

Pilea hyalina Fenzl. 2227, 4688, 9630, 11790.

Pilea microphylla (L.) Liebm. 1762, 4498, 5298, 8122, 16114, 16923, 17647, 20858, 21694, 22534, 23255, 24684, 24796, 25637.

Pilea pubescens Liebm. 3982.

Pouzolzia guatemalana (Blume) Wedd. 6266, 9125.

Pouzolzia occidentalis (Liebm.) Wedd. 4212, 10606, 13862, $13884,15432$. 
Urera caracasana (Jacq.) Gaudich. ex Griseb. 767, 3737bis, 10115, 10235, 10274, 11625, 13857, 13889, 17066.

Urtica chamaedryoides Pursh. 2586, 4900, 7559, 23160.

Urtica dioica L. 824, 825.

Urtica spiralis Blume. 792, 17042, 18841.

Urtica subincisa Benth. 2844.

Urtica urens L. 26689.

\section{Family Verbenaceae}

Aloysia citriodora Paláu. 22965.

Aloysia coalcomana Siedo. 15766 (TYPUS).

Aloysia gratissima (Gillies \& Hook.) Tronc. 16615, 18034, 21103, 21380, 22369, 22705, 23329, 25793.

Aloysia macrostachya (Torr.) Moldenke. 16632, 16895, 17482, 20782, 20936, 21054, 21227, 21791, 23049, 23615, 23852, 24066, 29446.

Aloysia wrightii (A. Gray) Heller. 18596.

Bouchea dissecta S. Watson. 6492, 12019, 12114.

Bouchea longiflora (Moldenke) G.L. Nesom. 29296.

Bouchea nelsonii Grenzeb. 10862.

Bouchea prismatica (L.) Kuntze. 1152, 1244, 4415, 6368, 10605, 12050, 12998, 13036, 13968, 23062, 23496.

Bouchea spathulata Torr. 16504, 25733, 27502.

Citharexylum affine D. Don. 317, 2042, 2367, 7344 (Type of Citharexylum affine var. glanduliferum Moldenke), 9011, 12631, 13738, 13739, 14082.

Citharexylum berlandieri B.L. Rob. 17934.

Citharexylum brachyanthum (A. Gray) A. Gray. 16525, 16554, 25828, 27633, 28312.

Citharexylum glabrum (S. Watson) Greenm. 14825.

Citharexylum hidalgense Moldenke. 15606.

Citharexylum hintonii Moldenke. 3209, 3399 (TYPUS), 7514, 7534, 7661.

Citharexylum mocinnoi D. Don. 5393, 6057, 8744.

Citharexylum ovatifolium Greenm. 2896.

Glandularia bipinnatifida (Nutt.) Nutt. 29875.

Glandularia alejandrana B.L. Turner. 17320, 19126, 19503, 25345, 27025 (TYPUS), 27199, 27582, 27636, 28692, 29144.

Glandularia bipinnatifida (Nutt.) Nutt. 4362, 11914, 13125, 16640, 16784, 16793, 17130, 17254, 19492, 19545, 20700, 25782, 27605, 28285.

Glandularia elegans (Kunth) Umber. 17356, 18515, 22445, 22731, 23928, 24037, 24402, 24808, 27959, 29029.

Glandularia polyantha Umber. 19342, 24132, 25054.

Glandularia pumila (Rydb.) Umber. 869, 2893, 5589, 7466, 8988.

Glandularia teucriifolia (M. Martens \& Galeotti) Umber. 4398, 8011 (Type of Verbena rinconensis Moldenke), 16788, 18286, 27670, 28928, 29149.

Glandularia turneri G.L. Nesom. 21007, 29191.

Lantana achyranthifolia Desf. 1216, 1765, 6006, 12123, 12301, 12462, 13093, 13961, 16780, 16901, 17933, 19336, 19456, 20034, 20395, 20530, 21136, 21180, 21406, 22468, 23380, 24200, 24284, 27518, 27523, 28844.

Lantana camara L. 518, 734, 832, 1058, 1187, 1226, 1233, 2590, 3158, 4429, 5280, 5357, 6366, 6372, 6373, 6455 (Туре of Lantana frutilla Moldenke), 6503, 6829, 6981, 9149,
9521, 10595, 11830, 12033, 12104, 12125, 12315 (Type of Lantana scandens Moldenke), 12693, 13005, 13034, 13844, 13852, 15183, 17595, 17682, 20010, 20054, 20333, 23293, 24099, 24628, 26506, 27754.

Lantana canescens Kunth. 3759, 18139, 22806, 24161, 28175.

Lantana hintonii Moldenke. 9307 (TYPUS).

Lantana urticoides Hayek. 27517.

Lippia graveolens Kunth. 13162 (Type of Lippia graveolens fma. macrophylla Moldenke), 16571, 28852.

Lippia alba (Mill.) N.E. Br. ex Britton \& P. Wilson. 1162, 1812, 4101, 5720, 9242, 13149, 13585, 13962, 14024.

Lippia graveolens Kunth. 2655, 5269, 6370, 6902, 13061, 13318, 23346, 23564, 25652, 27475, 28765.

Lippia umbellata Cav. 315, 393, 2099, 2330, 2574, 2620, 2918, 2921, 2989, 3057, 7237, 9838, 11100, 11156, 11302, $11633,11711,11717,11910,12383,12791,12945,12949$, 13482, 13550, 13614, 14868, 15706.

Petrea volubilis L. 14135.

Phyla dulcis (Trevir.) Moldenke. 764, 12257, 12968, 13798, 14055.

Phyla nodiflora (L.) Greene. 4343, 5695, 5810, 6028, 10575, 12037, 13823, 14523, 16650, 17833, 18631, 25822, 26503. Phyla strigulosa (M. Martens \& Galeotti) Moldenke. 6024 (Type of Phyla yucatana var. parvifolia Moldenke), 17722, 20330, 20601, 21575, 22062, 22188, 24113, 24414, 25318. Priva aspera Kunth. 4459, 4813, 4845, 9601, 10687, 11517 , 12170, 13154, 15625.

Priva grandiflora (Ortega) Moldenke. 13204.

Priva lappulacea (L.) Pers. 2643, 4360, 5801, 10366, 10545, 10573, 12224, 13062, 13941, 13975, 14391, 17664.

Priva mexicana (L.) Pers. 17675, 18010, 18720, 20565, 21201, 21219, 21553, 23070, 23390, 23479, 23497, 24844, 28890, 28956.

Stachytarpheta acuminata DC. 9366.

Stachytarpheta albiflora DC. 2078, 2134.

Stachytarpheta frantzii Pol. 13322.

Stachytarpheta hintonii Moldenke. 8236 (TYPUS).

Stachytarpheta mexicana Moldenke. 4504 (TYPUS), 8091.

Stachytarpheta mutabilis (Jacq.) Vahl. 10229, 13032, 14151.

Stachytarpheta velutina Moldenke. 1801 (TYPUS), 6725, 7783, 13000.

Tamonea curassavica (L.) Pers. 21464, 25519.

Verbena canescens Kunth. 29629.

Verbena carolina L. 29730, 29839.

Verbena gracilis Desf. 29716, 29868.

Verbena canescens Kunth. 16636, 17526, 18506, 18976, 19540, 19697, 21163, 21386, 21911, 24363, 27577, 28688.

Verbena carolina L. 822, 2729, 9287, 12974, 13022, 22232, 25399, 26017, 26845, 28286.

Verbena ehrenbergiana Schauer. 17609, $21398,23827$.

Verbena falcata G.L. Nesom. 20796, 28113 (TYPUS).

Verbena gracilis Desf. 5847.

Verbena johnstonii (Moldenke) G.L. Nesom. 18241, 18487, 21853, 23586, 25501, 27065, 27464, 27938, 28025, 28892, 29111, 29451.

Verbena litoralis Kunth. 1575, 3731, 12520, 13965 (Type 
of Verbena litoralis var. albiflora Moldenke), 19997, 20325, 20345, 21266, 21268, 23269, 24171, 24343.

Verbena madrensis Nesom. 20329, 24216, 24412, 24440, 25422.

Verbena menthifolia Benth. 11991 (Type of Verbena hintonii Moldenke).

Verbena neomexicana (A. Gray) Small. 17695.

Verbena recta Kunth. 4607, 17407, 18566.

Verbena scabra Vahl. 26191, 26653.

\section{Family Violaceae}

Hybanthus pennellii (C.V. Morton) B.L. Turner. 25372, 27402, 29053.

Pombalia attenuata (Humb. \& Bonpl. ex Willd.) Paula-Souza. 1260, 1269, 1481, 4210, 4431, 4543, 6335, 6431, 9103, 9124, 13088, 13978.

Pombalia oppositifolia (L.) Paula-Souza. 1250, 4341, 4571. Pombalia verbenacea (Kunth) Paula-Souza. 20562, 20590, 21402, 21452, 21666, 22029, 22056.

Viola flagelliformis Hemsl. 22971.

Viola galeanaensis M.S. Baker. 17102, 17394, 18954, 20312, 22390, 22752, 22841, 24081.

Viola grahamii Benth. 907, 1145, 3496, 11958.

Viola hookeriana Kunth. 22678, 26529, 26556.

Viola humilis Kunth. 492, 3993.

Viola oxyodontis H.E. Ballard. 4986, 6167 (TYPUS), 7940, 10479, 13876, 14374.

Viola tricolor L. 799.

\section{Family Vitaceae}

Ampelocissus acapulcensis (Kunth) Planch. 3357, 3401, 5951, 13979.

Ampelopsis denudata Planch. 5717, 7244, 7537, 10038.

Cissus tuberosa DC. 8172.

Cissus alata Jacq. 748, 6287, 6542, 9594, 10231, 14477.

Cissus cucurbitina Standl. 14675.

Cissus trifoliata (L.) L. 16568.

Cissus tuberosa DC. 7818.
Cissus verticillata (L.) Nicolson \& C.E. Jarvis. 660, 768, 2292, 2876, 2976, 3812, 3900, 4025, 4215, 5862, 6453, 6537, 6983, 10042, 10199, 10784, 12635, 13577, 16028.

Parthenocissus quinquefolia (L.) Planch. 3936, 17723, 21685.

Vitis berlandieri Planch. 19213.

Vitis cinerea (Engelm.) Engelm. ex Millardet. 19138, 22824, 24073, 24141, 24299.

Vitis tiliifolia Humb. \& Bonpl. ex Roem. \& Schult. 482, 3115, 3730, 7639, 13543.

\section{Family Winteraceae}

Drimys granadensis L. f. 14235, 14441.

\section{Family Xanthorrhoeaceae}

Aloe vera (L.) Burm. f. 5726.

Asphodelus fistulosus L. 16608, 19412, 23129.

\section{Family Xyridaceae}

Xyris mexicana S. Watson. 957.

\section{Family Zingiberaceae}

Hedychium coronarium J. König. 4525.

Renealmia pacifica (Maas) Maas \& H. Maas. 10591.

Zingiber officinale Roscoe. 16007.

\section{Family Zygophyllaceae}

Fagonia scoparia Brandegee. 28842.

Guaiacum angustifolium Engelm. 25798.

Guaiacum coulteri A. Gray. 5641, 6458.

Kallstroemia grandiflora Torr. 5487, 6480, 7116, 23558, 24744, 28829, 29433.

Kallstroemia hintonii D. Porter. 12106 (TYPUS).

Kallstroemia maxima (L.) Hook. \& Arn. 1061, 4763, 5433, 5613, 6278, 6304, 8153, 9127, 10499, 13981, 14575.

Kallstroemia parviflora Norton. 9119, 12097, 16869, 20591, 21433, 23038, 23545, 25461, 25865, 28664, 28790.

Kallstroemia pubescens (G. Don) Dandy. 5789, 14543.

Kallstroemia rosei Rydb. 13050.

Larrea tridentata (DC.) Coville. 16557, 23993.

Porlieria angustifolia (Engelm.) A. Gray. 19279.

Sericodes greggii A. Gray. 23187, 27419.

Tribulus cistoides L. 29906.

Tribulus cistoides L. 5458, 7115. 